
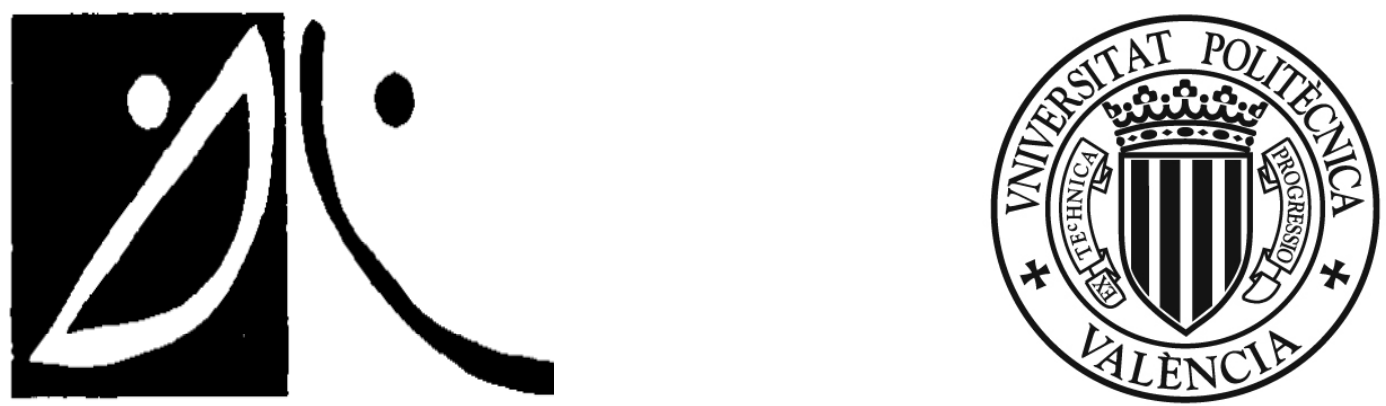

Universitat Politècnica de València

Departamento de Comunicaciones

\title{
Towards Compact and \\ High Speed Silicon Modulators
}

\author{
Antoine Brimont
}

Supervisor: Dr. Pablo Sanchis Kilders

Tesis presentada para la obtención del grado de Doctor en Telecomunicaciones de la Universitat Politècnica de València.

Thesis submitted to the Universitat Politècnica de València in partial fulfillment of the requirements for the degree of Doctor of Philosophy in Telecommunications engineering. 





\section{Preface}

"Photonics" is the field of science that involves the manipulation of light, i.e. photons. A photon is the smallest quantity (quantum) of electromagnetic radiation and has neither mass nor electric charge. The idea of using coherent light beams for the good of society emerged in the 60's by creating the first semiconductor laser, then followed by the first optical fiber and erbium doped fiber amplifier in the 70's. At the beginning of the 80 's, the term "photonics" became commonly used among the scientific community and the combination of efficient light emitters, optical modulators, amplifiers, switches, optical fibers and detectors, paved the way for the late $20^{\text {th }}$ century telecommunication revolution: the Internet. Meanwhile, photonics has also been showing great success in many other fields of applications such as high power lasers, biological and chemical sensing, medical diagnosis and therapy as well as display devices and therefore gathers many different aspects in terms of optical engineering applications. In the 90 's and at the beginning of the $21^{\text {st }}$ century, two prefixes, "micro" and "nano", were successively added to this word. They basically arise from the fact that light can be manipulated at micro- and more recently nano-scale owing to the ceaseless progress in nanofabrication tool development. As a result, photonic components, so far discretely fabricated and assembled, are on the verge of experiencing drastic changes by being integrated monolithically onto miniature integrated chips due to their ever decreasing footprints. Although it is widely accepted that III-V compounds, owing to their superior optical properties for light emission, modulation and detection, are materials of choice for such a purpose, silicon, known as the fundamental material of electronics, was proposed in the early 90's as an alternative photonic material despite its relatively poor active optical properties. Therefore, a natural question arises: why exploring silicon photonics? First, because silicon is transparent in the near-infrared range, and is consequently a good candidate for short-range telecommunication wavelength 
guidance. Moreover, it is the second most abundant element in Earth's crust after oxygen and finally, it is far cheaper and easier to process than its III-V counterparts. However, then, why would one want to confine and control photons on silicon chips if electrons have successfully fulfilled their role so far, enabling us to enjoy our modern "electronic way of life"? The answer to this is that the electronic roadmap is not expected to be eternally following Moore's law and silicon chips will sooner or later require, at least in the mid-term, some added functionalities that could likely be provided by monolithically integrated photonic devices. The idea behind this is to benefit from the well established maturity of silicon-based electronics and realize similar/complementary functions so electrons and photons could function in harmony on small, inexpensive, energy efficient and fast silicon chips. As a result, powerful and cheap supercomputers, sensors, highly efficient medical diagnosis tools could be created and revolutionize drastically our society. Two decades of advanced research in silicon photonics has shown that silicon and silicon-based devices could guide, detect, switch, modulate and unexpectedly emit near-infrared light. However, despite this incredibly fast progress, comprehensive knowledge of the physics and technology of silicon photonics-based key functionalities is still required. This thesis is a humble contribution to the field of silicon photonics through demonstrating high speed modulation of near infrared light in silicon devices featuring ultra-small footprints.

I hope this work will be a useful reference for future investigations on silicon modulators and silicon photonic devices in general, and will eventually helps in accelerating the foreseeable commercial deployment of low cost and high performance silicon nanophotonic chips for a wide variety of applications.

Because this work is obviously not the result of a single scientist working on his own, I would like to thank all the people (sorry if I forgot someone...) who have contributed to its realization.

Thank you, Pablo, for being a very good supervisor. By "very good", I mean someone who gives you the opportunity to express your potential by orientating and advising you especially when you are drowning either in the laboratory or in front of your never ending 3D FDTD simulations. Thank you for having taken the time to read entirely this thesis and for having provided useful comments. Thank you also for having been patient and allow me to finish my PhD "nearly-on-time". I have been and still am very proud of forming part of your research team. 
Thank you, Jose Vicente, for having shared these few years and especially for having made me laugh in the "darkness" of our nanophotonic characterization laboratory with your (short) jokes that I am recycling over and over. I wish you a very successful and peaceful life.

Thank you Ana, Mariam and Sara for helping me out with the "resumen" and "resum" respectively in Castilian and Valencian, and for showing such a great interest in working towards your respective PhDs. I encourage you to sustain you enthusiasm.

Thank you, Claudio and Javier Herrera, for your great support in the laboratory and for being always available. It is a pleasure to work with such experienced and nice colleagues.

Thank you, Alejandro, for pushing us all to become recognized researchers. I really think you are doing a great job as an area manager. Thank you also for having taken the time to read entirely this thesis and for having provided useful comments.

Thank you, Javier Marti, for giving me the opportunity to work in your advanced research institute. It is providing me a very stimulating environment where I can simultaneously fulfill my passion for science and do it for a living.

Thanks to the fabrication and packaging teams for their countless hours of dedicated work to make our (sometimes impossible) designs become a reality on tiny silicon chips. Thank you, Francisco, Amadeu, Jose Ayucar, Juan, Laurent, Nuria, Gianni, Mercé, Antonio, José Alfredo, Glenn and Luis.

Thanks also to all my office mates, Carlos, Rubén, Pak, Javier García, Veronica, Jaime, Ruth, Dani, Joaquín, Jose Maria, Ico, Andreas, Clara, Ingrid, Jordi, David, Guillermo, Pere, Maria, Marta...) who have been present during my $\mathrm{PhD}$ and who have contributed directly or indirectly to this work via fruitful discussions and advices.

Thanks to the ePIXnet, and HELIOS, DEMOTEC people for allowing me to enter the silicon photonics community, and for providing me an extremely suitable environment to complete my $\mathrm{PhD}$.

In particular, thank you Jean-Marc, Laurent, Maryse, and the CEA-LETI team for fabricating such highly complex devices and for your advices on the fabrication process as well as for your great support during the project.

Thanks to Wim, Pieter and Günther from Ghent University, for showing me how to couple light vertically to silicon chips and for introducing me to IPKISS. 
Thanks to Fred and Dave from the University of Surrey. You guys really contributed to introduce me to the field of silicon modulators and I have really enjoyed our fruitful collaborations and friendship. Thank you, Graham Reed for your useful inputs and valuable experience in the field of silicon photonics.

Thank you, Gilles, Delphine and Laurent from the Université Paris Sud, for our collaborative work. It was a pleasure to share our respective know-how and dynamism.

Outside my work, I would like to thank first my parents, Mamam, Papa, for having provided me a suitable affective and cultural environment as well as financial support to make it through university. Thanks to my brother and sister Guillaume and Marine, as well as my brother and sister in-law, Carine and Brieuc, for showing interest in what I have been working on during my PhD. Thank you, Claude, for sharing your highly relevant knowledge on the world of research. Thank you, Anne-Marie for your generosity and support. Thanks to my grand-parents Nanou, Pajacques, Papy and our well missed mammy for your affective (and also financial) support and for sharing your lifetime experience and knowledge. Thanks to my parents in-law Rache, Joro, and my brother in-law, Zhivko for helping out whenever they could and for introducing me to the magnificent shopska salata, which has kept me healthy during my PhD. Thanks also to Antonin, Damien, Nicolas, Axel, François and Anne-Barbara for those moments we shared on a basketball court as part of a great team.

Finally, last but not least, I would like to thank you, Plamena, my loving wife, for helping me keep my feet on the ground (especially after a long day in the laboratory) and providing me a wonderful and warm environment to complete my $\mathrm{PhD}$ in the best possible conditions. Thank you, Gabriel, our wonderful baby boy for filling us with happiness.

Antoine Brimont, Valencia, 31 October 2011 


\section{Resumen}

Los moduladores son elementos claves para la transmisión de la señal y el procesamiento de la información. Las técnicas de fabricación avanzadas "complementary metal-oxide semiconductor" (CMOS) permiten reducir drásticamente las dimensiones de estos dispositivos de interés para la implementación a gran escala en un chip de silicio a bajo coste. El trabajo realizado en esta tesis se centra en el diseño, la fabricación y la caracterización de estructuras de onda lenta con el objetivo de realizar moduladores compactos y eficientes integrados en un chip de silicio. El trabajo se divide en cuatro capítulos y un capítulo de conclusión y perspectivas. El capitulo uno introduce los fundamentos de física del estado sólido y de los mecanismos básicos de propagación guiada de la luz por reflexión total interna. El capítulo dos presenta los parámetros importantes de los moduladores electro-ópticos así como un trabajo de recopilación de todos los mecanismos físicos que pueden ser empleados para modular la luz en silicio. Además, se presenta el estado del arte de los moduladores basados en silicio. El capítulo tres presenta el diseño, fabricación y caracterización de un modulador electro-óptico en silicio compacto y eficiente basado en el efecto de onda lenta en una estructura periódica unidimensional integrada, cuya geometría, similar a la de una red de Bragg, permite reducir la velocidad de grupo de un paquete de ondas. Dicho efecto, se emplea para incrementar la interacción luz-materia y por lo tanto la eficiencia del modulador electro-óptico. El capítulo cuatro demuestra experimentalmente que dicha guía unidimensional periódica puede ser mejorada a fin de conseguir que el efecto de baja velocidad de grupo suceda en un rango mayor de longitudes de onda para posibles aplicaciones como la multiplexación por división de longitud de onda. En el capítulo cinco, se proporcionan conclusiones y perspectivas sobre el trabajo realizado. 


\section{Resum}

Els moduladors són elements clau per a la transmissió del senyal i el processament de la informació. Les tècniques de fabricació avançades "complementary metaloxide semiconductor" (CMOS) permeten reduir dràsticament les dimensions d'estos dispositius d'interés per a la implementació a gran escala en un xip de silici a baix cost. El treball realitzat en esta tesi es centra en el disseny, la fabricació i la caracterització d'estructures d'ona lenta amb l'objectiu de realitzar moduladors compactes i eficients integrats en un xip de silici. El treball es dividix en quatre capítols i un capítol de conclusió i perspectives. El capítol u introduïx els fonaments de física de l'estat sòlid i dels mecanismes bàsics de propagació guiada de la llum per reflexió total interna. El capítol dos presenta els paràmetres importants dels moduladors electroòptics així com un treball de recopilació de tots els mecanismes físics que poden ser empleats per a modular la llum en silici. A més, es presenta l'estat de l'art dels moduladors basats en silici. El capítol tres presenta el disseny, fabricació i caracterització d'un modulador electroòptic en silici compacte i eficient basat en l'efecte d'ona lenta en una estructura periòdica unidimensional integrada, la geometria de la qual, semblant a la d'una xarxa de Bragg, permet reduir la velocitat de grup d'un paquet d'ones. El dit efecte, s'empra per a incrementar la interacció llum-matèria i per tant l'eficiència del modulador electroòptic. El capítol quatre demostra experimentalment que dita guia unidimensional periòdica pot ser millorada a fi d'aconseguir que l'efecte de baixa velocitat de grup succeïsca en un rang major de longituds d'ona per a possibles aplicacions com la multiplexació per divisió de longitud d'ona. En el capítol cinc, es proporcionen conclusions i perspectives sobre el treball realitzat. 


\section{Abstract}

Optical modulators are key building-blocks for high speed signal transmission and information processing. Current "complementary metal-oxide semiconductor" (CMOS) advanced fabrication tools allow for a dramatic reduction in device size to achieve large scale and cost effective integration. The work developed in this thesis is essentially focused on the design, fabrication and characterization of slow wave structures in order to realize compact and efficient modulators integrated on ultra-small silicon chips. This thesis consists of four main chapters as well as a concluding section on the work accomplished. Chapter one provides a short background on solid state physics and light propagation in high contrast integrated waveguides in order to give basic tools for understanding the underlying physics behind silicon electro-optical modulator operation. Chapter two introduces the main parameters of electro-optical modulators and is followed by a description of the main physical mechanisms that may be used for optical modulation in silicon. The state-of-the-art of silicon and silicon-based modulators is also provided. Additionally, the passive and active characterization techniques and setups are presented. Chapter three is devoted to the design, fabrication and characterization of an ultra-fast and compact slow wave, plasma dispersion-based silicon electro-optical modulator. Chapter four is dedicated to the design, characterization and fabrication of a novel type of engineered silicon slow wave waveguide exhibiting a high group index over a wide wavelength range. Potential applications of such a waveguide for wavelength-division-multiplexing (WDM) high speed modulation are explored. Finally, conclusions on the work realized are provided in Chapter five. 


\section{Table of Contents}

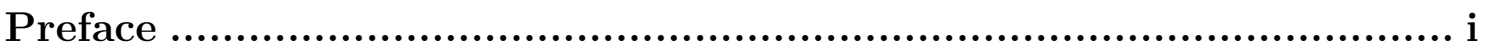

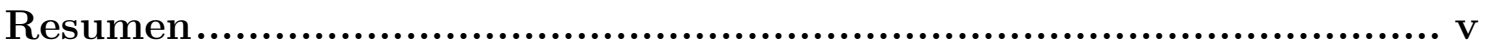

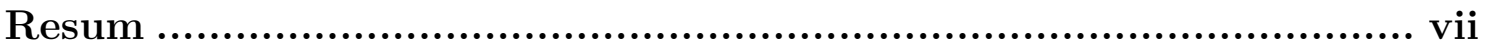

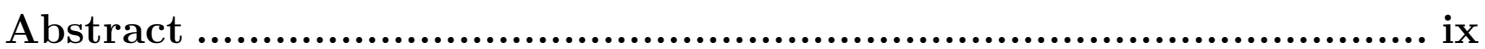

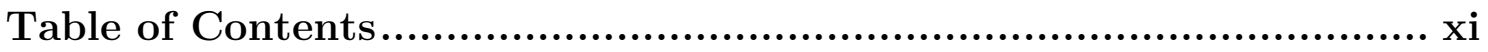

Introduction: Challenges and Opportunities for Silicon Photonics ......xv

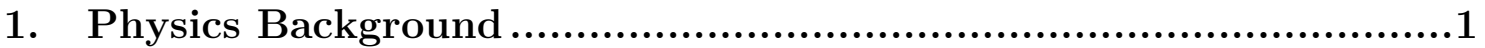

1.1 Fundamentals on Solid State Physics....................................... 1

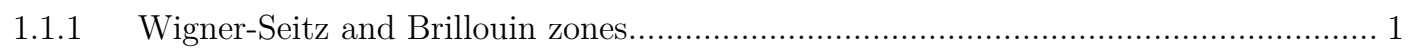

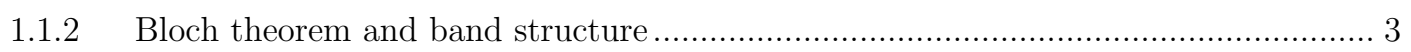

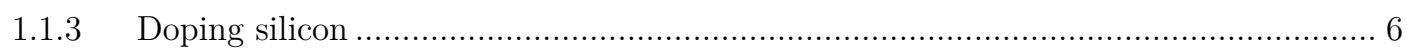

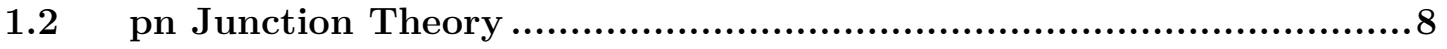

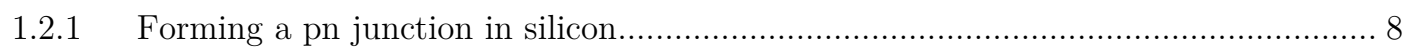

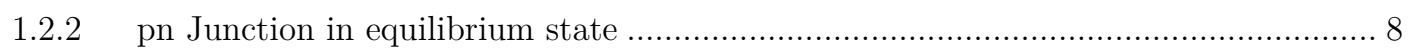

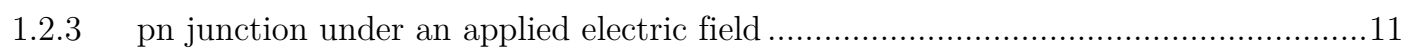

1.2.4 Ideal current-voltage characteristics: Shockley's equation.........................................13

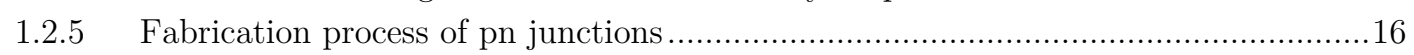

1.3 Fundamentals of Light Propagation in Optical Waveguides............17

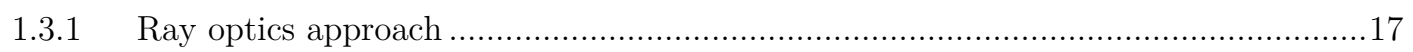

1.3.2 Extension of the ray optics approach …….......................................................18

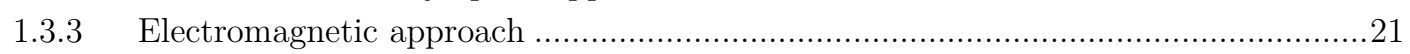

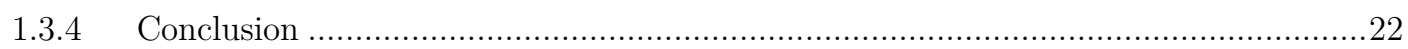

2. Optical Modulators .....................................................23

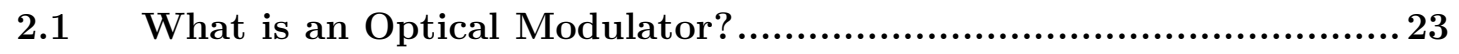

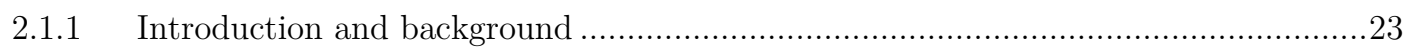

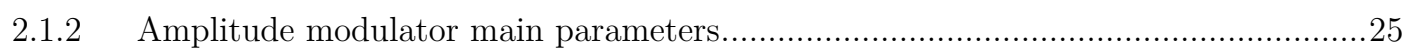

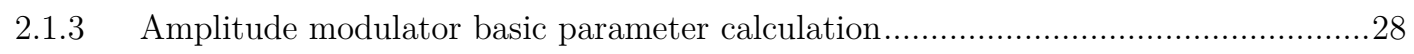

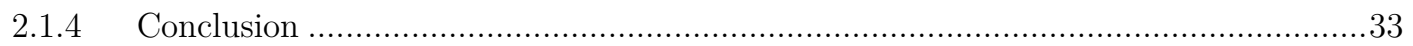

2.2 Silicon as an Efficient Material for Optical Modulation...................34

2.3 Physical Mechanisms for Optical Modulation in Silicon .................. 34

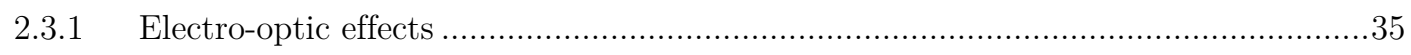

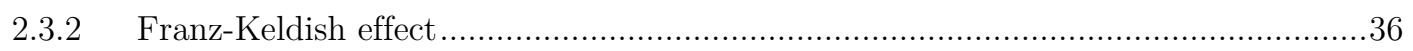




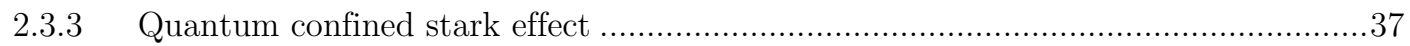

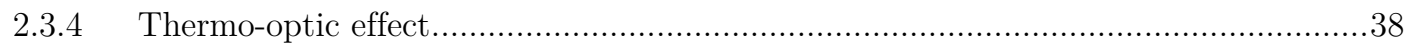

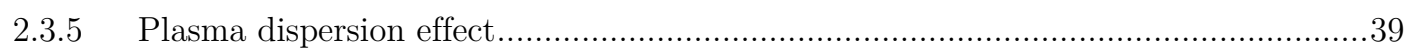

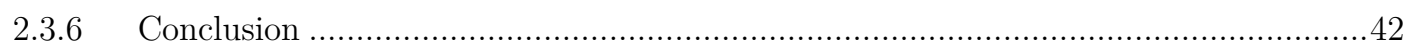

2.4 Silicon-Based Modulators: The State-of-the-Art ......................... 43

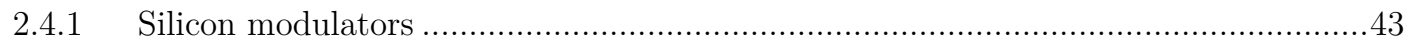

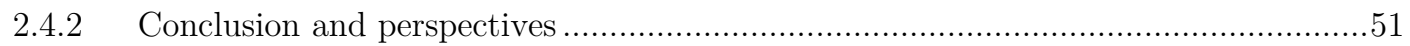

2.5 Optical Modeling Tools ......................................................... 51

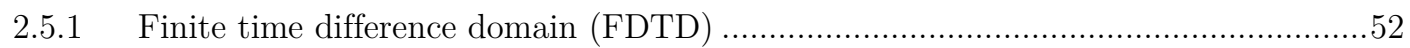

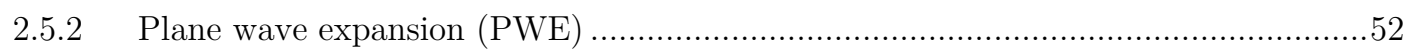

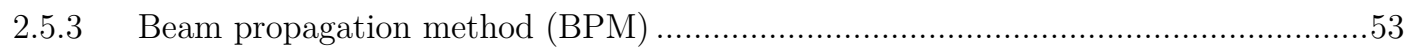

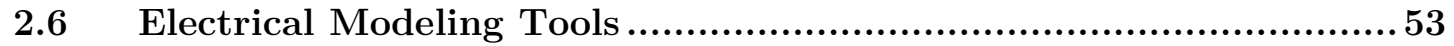

2.6.1 Semiconductor device simulation framework: ATLAS ……....................................53

2.7 Electro-Optical Modeling Convergence ....................................5 54

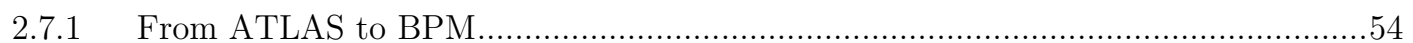

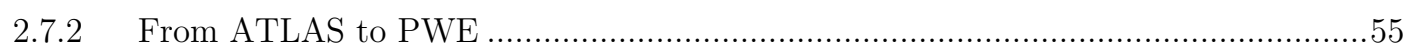

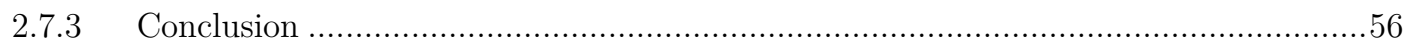

2.8 Passive and Active Characterization Setups …......................... 57

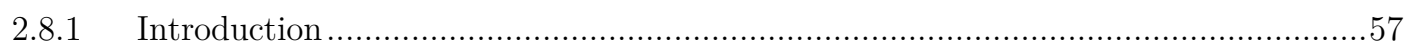

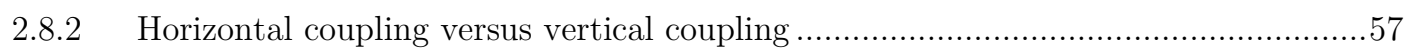

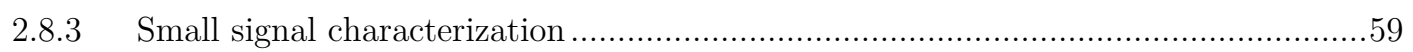

2.8.4 Data transmission and bit error rate measurements ................................................61

3. Compact Depletion-Based Slow Wave Modulator ....................63

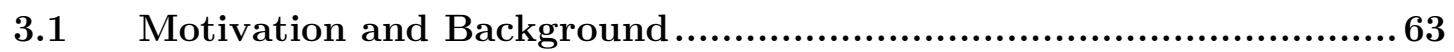

$3.2 \quad$ Target Applications of Slow Wave Structures .............................6 65

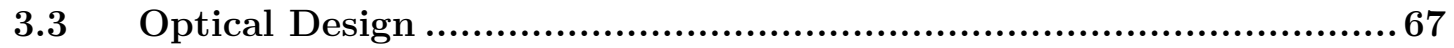

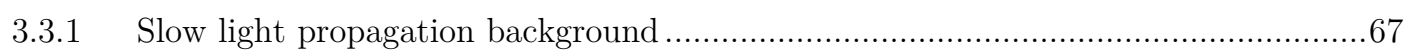

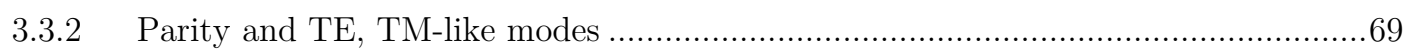

3.3.3 Slow wave structure choice............................................................................ 70

3.3.4 Slow wave structure overview............................................................................

3.3.5 Parity and TE, TM-like modes in the shallow-etched slow light structure ...............78

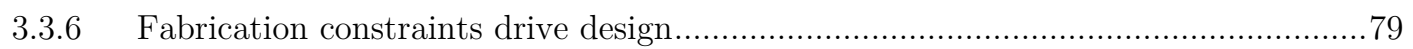

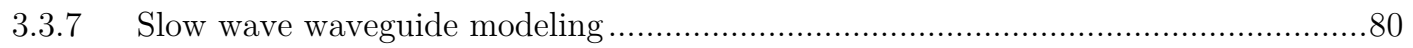

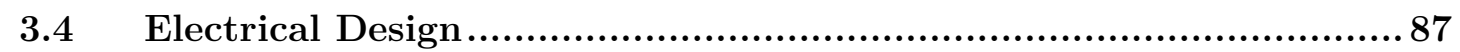

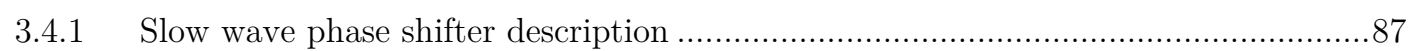

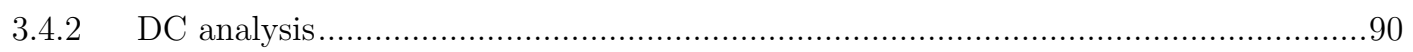

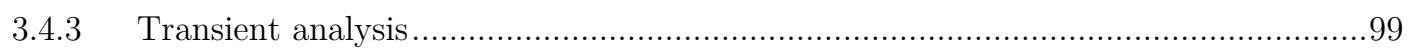

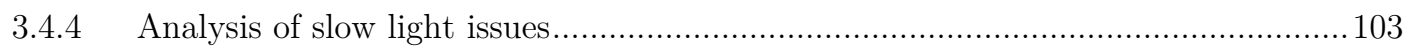

3.4.5 Slow light propagation and 3dB roll-off bandwidth limitations ............................. 119

3.5 Evolution of the Slow Wave Modulator Electrical Design ............. 121

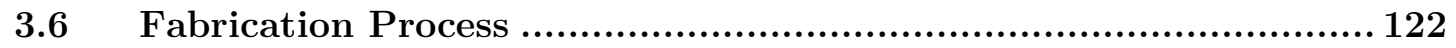

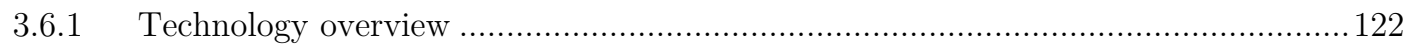

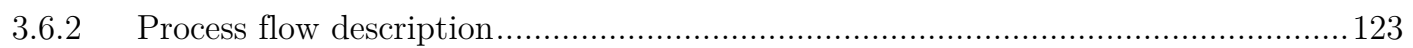




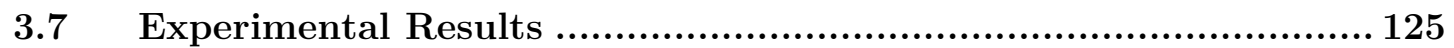

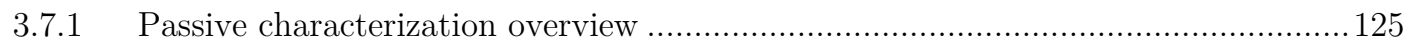

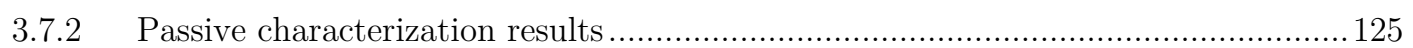

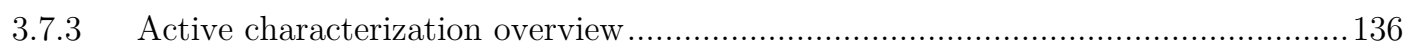

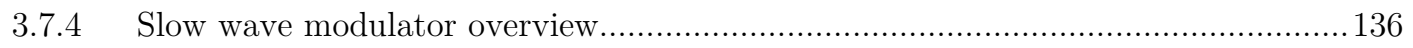

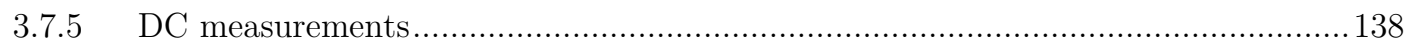

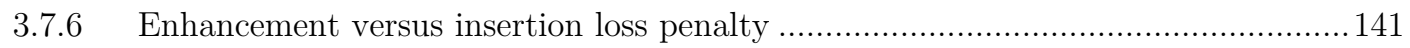

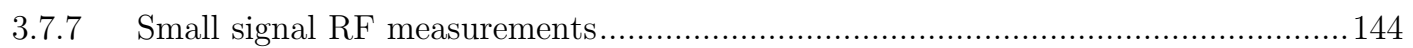

3.7.8 Eye diagrams and bit error rate measurements....................................................... 151

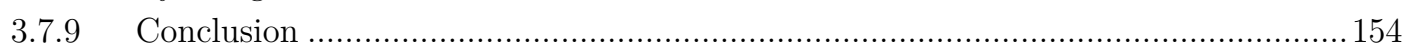

4. Group Index Engineering in Silicon Slow Wave Structures ....... 155

4.1 Motivation and Background .................................................... 155

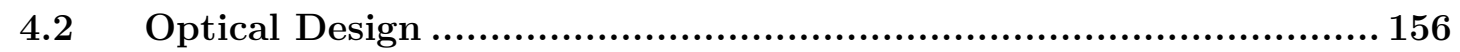

4.2.1 Engineered slow wave structure overview …............................................ 156

4.2.2 Group index engineering guidelines ............................................................. 157

4.2.3 Modeling of the engineered laterally corrugated waveguides ........................... 158

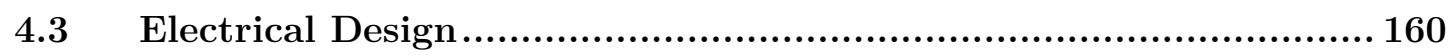

4.3.1 DC analysis of a slow wave phase shifter with broad optical bandwidth...............160

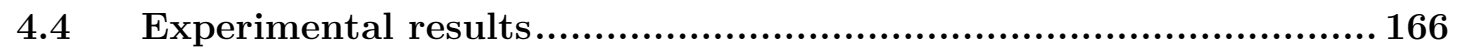

4.4.1 Passive characterization results ................................................................. 166

4.4.2 Efficient coupling to the slow light mode .................................................... 169

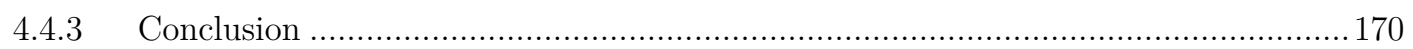

5. Conclusions and Prospects .......................................... 173

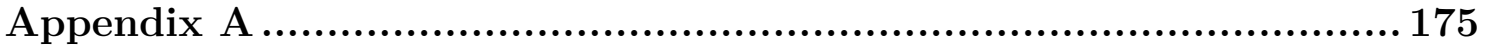

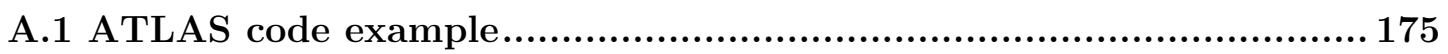

A.2 Conversion code from ATLAS to BEAMPROP/BANDSOLVE........ 176

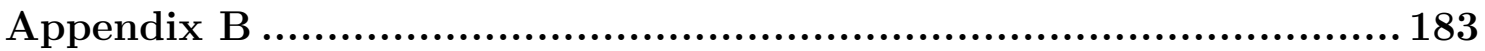

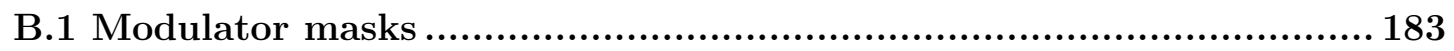

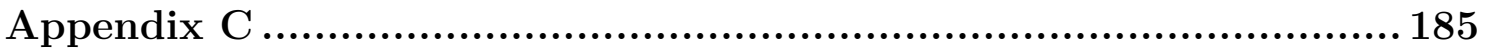

C.1 Publications in peer-reviewed journals........................................ 185

C.2 Other contributions in peer-reviewed journals .............................. 186

C.3 National and international conferences ......................................... 186

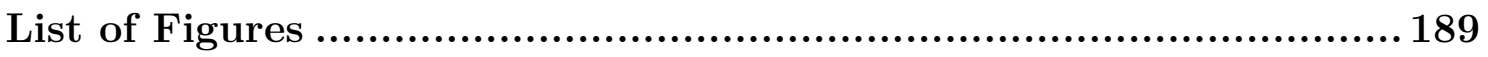

List of Tables............................................................... 201

Acronyms ................................................................... 203

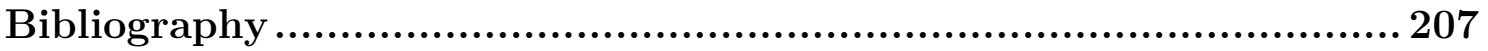




\section{Introduction: Challenges and Opportunities for Silicon Photonics}

Since the creation of the first semiconductor laser diode in 1962 at General Electric (NY, USA), photonics, the science of generating, modulating, guiding processing and detecting light has been coming up to the forefront of applied science and commercial deployment to face the ceaselessly increasing demand for communication networks at higher data rates, a reality initially triggered by the late $20^{\text {th }}$ century telecommunication revolution: the Internet. On the other hand, since the first transistor was discovered at Bell Labs (NJ, USA) in 1947, electronics, known as the "electron science" has been experiencing tremendous progress and undeniably won its spurs by creating the mould of our society's technological evolution. However, it is currently known that both fields are facing significant challenges.

On one hand, the complexity, cost and size of fiber-based discrete optical components have been slowing down the exponential progress of photonics in some applications fields. On the other hand, electronics is foreseeably reaching fundamental physical limitations. The fact is, Moore's law predicted that the number of transistors on a single silicon chip would double every two years, which, so far has been well sustained owing to the advancement in electronic technology that is continuously enabling the realization of high performance transistors with ever decreasing footprints. However, unavoidable limitations appear as the packing density of these fundamental building blocks becomes extreme. Indeed, the power dissipation in a single processor increases as the density of transistors does and is becoming challenging to harness on a few millimeter square chips, especially because the amount of leakage power radiated in the form of heat grows faster than the useful power. In addition to this, the 
total copper interconnection length increases in large scale integration systems, leading to more latency and RC delay in the signal transmission $[1,2]$. Still, it is believed that advanced engineering will allow electronic roadmap to be further extended for at least a decade. One provisional alternative consisting in three dimensional chip stacking [3], is expected to reduce the on-chip electrical interconnection latency. Another alternative, which has received growing interest in the nanoelectronics community, is the use of graphene as an alternative material. This one atomic-monolayer material features superior electrical properties such as two order of magnitude greater electron mobility than that found in silicon. As a consequence, graphene-based molecular transistors operating in the 500 to $1000 \mathrm{GHz}$ range [4, 5] have been reported. However, for the time being and in the mid-term, the most promising option to mitigate the foreseeable electronic bottleneck is the use of short-range optical interconnects. Integrated photonics or "nanophotonics" is expected to provide a very powerful, inexpensive and energy efficient platform to increase the interconnection bandwidth of processors themselves (intra-chip), between multiple cores processors (chip-tochip) and possibly boards (off-chip). This may be realized via the on-chip integration of photonic components whose dimensions are on the order of micron and sub-micron scales. So far, two main technological trends based on silicon and III-V compounds offer the potential of realizing basic optical functions. III-V compounds are widely used for light emission, modulation and detection due to their direct band gap and good optical properties. However, the complex and expensive fabrication processes required for such materials make large volume manufacturing quite challenging. Conversely, silicon, well known as the holy grail of electronics was proposed in the early 1990's [5-7] as an alternative material for photonics technological development due to its high level of integration and low assembly cost compared to its III-V counterparts. Furthermore, in opposition to optical fibers, the high refractive index contrast existing between silicon and silicon dioxide, which is a material usually employed as an insulator for electronic circuits, enables strong confinement of light via total internal reflection (TIR) in optical waveguide exhibiting sub-wavelength dimensions. For instance, current standard silicon-on-insulator (SOI) technology enables to make up to two order of magnitude tinier single mode waveguides compared to low index contrast standard single mode optical fibers (SMOF) with 8 to $10 \mu \mathrm{m}$ core diameters. Indeed, demonstrations of compact and low loss silicon passive building blocks [6, 7] using conventional CMOS microelectronic fabrication processes established the 
basis for efficient near-infrared light guidance in future optical devices. In the meantime, significant progress has been made in the field of silicon-based active devices in spite of silicon's indirect bandgap and transparency in the near-infrared range, making lasing and light detection at these wavelengths very inefficient. Furthermore, silicon's natural centro-symmetric crystalline structures rules out any linear electro-optic Pockels effect, conventionally used as a modulation mechanism in commercial lithium-niobate modulators, for instance. However, over the last decade, the advancement in realizing efficient silicon or silicon-based active components has shown that silicon photonics is reaching a degree of maturity never imagined so far. Indeed, the potential commercial impact is motivating research efforts towards creating highly reliable integrated silicon near-infrared lasers, optical modulators, detectors, and all types of passive elements such as multiplexers, demultiplexers, splitters, directional couplers and delay lines to eventually realize complex optical functions. The key to success is integrating all these building blocks on a standard CMOS-processed silicon wafer while achieving ultra-low footprints. Among them, optical modulators, which handle electrical-to-optical data conversion, are of paramount importance. This is precisely the main line of this work.

Chapter 1 provides a short background on solid state physics and light propagation in high contrast integrated waveguides in order to give basic tools for understanding the underlying physics behind silicon electro-optical modulator operation. Chapter 2 introduces the main parameters of electro-optical modulators and is followed by a description of the main physical mechanisms that may be used for optical modulation in silicon. The state-of-the-art of silicon and silicon-based modulators is also provided. Additionally, the passive and active characterization techniques and setups are presented. Chapter 3 is devoted to the design, fabrication and characterization of an ultra-fast and compact slow wave, plasma dispersion-based silicon electro-optical modulator. Chapter 4 is dedicated to the design, characterization and fabrication of a novel type of engineered silicon slow wave waveguide exhibiting a high group index over a wide wavelength range. Potential applications of such a waveguide for wavelengthdivision-multiplexing (WDM) high speed modulation are explored. Finally, conclusions on the work realized are provided in Chapter 5. 


\section{Chapter 1}

\section{Physics Background}

To facilitate understanding of the dominant mechanisms governing light modulation in silicon, this chapter takes a look back at the fundamentals of solid state physics [8]. The following section provides a starting point to identify the phenomena triggering charge carrier motion across silicon pn junctions. Furthermore, we provide an overview of light propagation in optical waveguides[9] based on the example of a simple planar configuration. Two approaches are presented: the ray optics picture and its extended version, which allows us to understand light propagation via TIR, and the electromagnetic approach using Maxwell's equations, which enables us to derive the guided modes and the corresponding field patterns.

\subsection{Fundamentals on Solid State Physics}

\subsubsection{Wigner-Seitz and Brillouin zones}

A crystal is a solid in which the constituent atoms, molecules, or ions are packed in a regularly repeated pattern extending in all three spatial dimensions. Let $\vec{a}_{1}, \vec{a}_{2}, \vec{a}_{3}$ be primitive vectors of a crystalline solid such that the crystal structure remains invariant under translation by any vector $\vec{R}$, being a linear combination of these basis vectors (i.e. multiplied individually by an integer, $n_{1}, n_{2}, n_{3}$, respectively). Each point of the Bravais lattice can hence be defined by the following set of vectors as illustrated in Figure 1-1.

$$
\vec{R}=n_{1} \vec{a}_{1}+n_{2} \vec{a}_{2}+n_{3} \vec{a}_{3}
$$




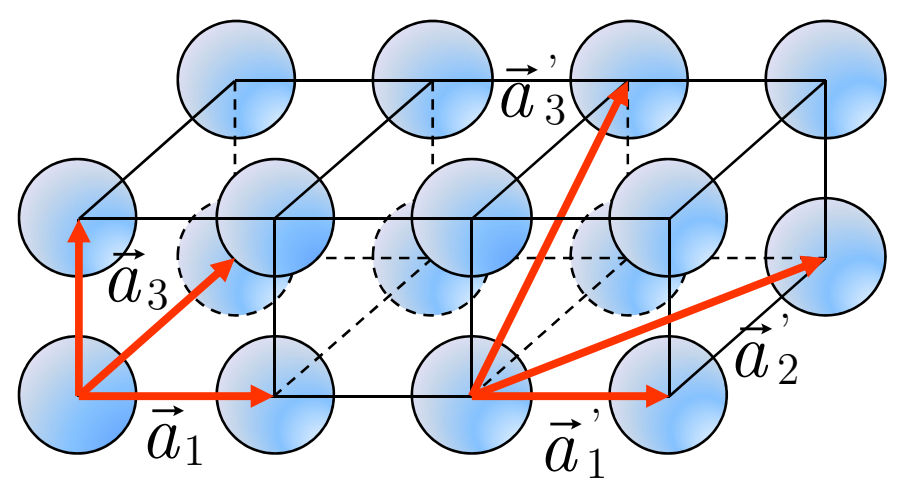

Figure 1-1: Examples of two random combinations of primitive vectors in a cubic lattice structure

Silicon crystallizes in a diamond lattice structure, which consist of two interpenetrating face-centered cubic lattices as depicted in Figure 1-2.

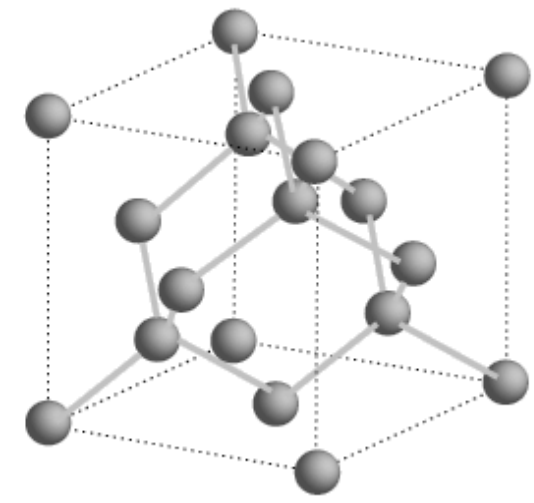

Figure 1-2: Diamond lattice structure

Once the primitive vectors of the direct lattice are chosen, a so-called reciprocal lattice is defined in order to make convenient the study of band structures and electron dynamics within the crystal. The primitive vectors $\vec{b}_{1}, \vec{b}_{2}, \vec{b}_{3}$, which generate the reciprocal lattice are:

$$
\vec{b}_{1}=2 \pi \frac{\vec{a}_{2} \times \vec{a}_{3}}{\vec{a}_{1}\left(\vec{a}_{2} \times \vec{a}_{3}\right)} \quad \vec{b}_{2}=2 \pi \frac{\vec{a}_{3} \times \vec{a}_{1}}{\vec{a}_{1}\left(\vec{a}_{2} \times \vec{a}_{3}\right)} \quad \vec{b}_{3}=2 \pi \frac{\vec{a}_{1} \times \vec{a}_{2}}{\vec{a}_{1}\left(\vec{a}_{2} \times \vec{a}_{3}\right)}
$$

The resultant reciprocal lattice vector is developed as a linear combination of the primitive reciprocal lattice vectors.

$$
\vec{K}=k_{1} \vec{b}_{1}+k_{2} \vec{b}_{2}+k_{3} \vec{b}_{3}
$$


Where $k_{1}, k_{2}, k_{3}$ are integers.

In solid state physics, it is usually convenient to define the so-called Wigner-Seitz cell. The Wigner-Seitz cell is build by drawing perpendicular bisector planes in the reciprocal lattice from the chosen centre to the nearest equivalent reciprocal lattice sites. Figure 1-3 shows the Wigner-Seitz cell of a diamond lattice structure in the reciprocal lattice also referred to as the first Brillouin zone.

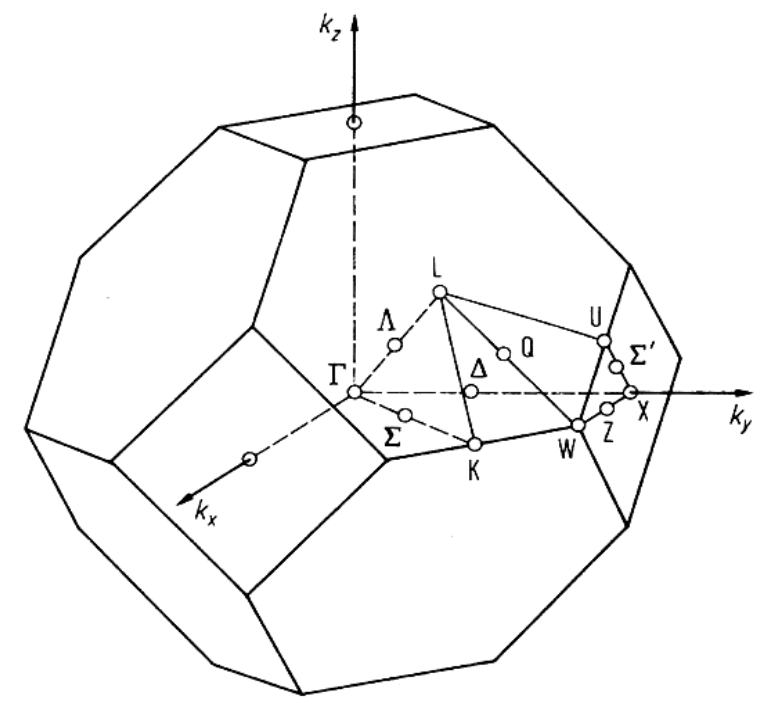

Figure 1-3: Brillouin zone of the diamond lattice structure

\subsubsection{Bloch theorem and band structure}

Electrical properties of semiconductors have been extensively studied and led to a complete description of some key semiconductor materials. The band structure of a crystalline solid, which links the energy of a single electron to its momentum, may be approximated by solving the time independent Schrödinger's Equation.

$$
\begin{gathered}
\left(-\frac{\hbar^{2}}{2 m} \nabla^{2}+U(\vec{r})\right) \Psi_{k}(\vec{r})=E_{k}(\vec{r}) \Psi_{k}(\vec{r}) \\
U(\vec{r})=U(\vec{r}+\vec{R})
\end{gathered}
$$

Where $\Psi_{k}(\vec{r})$ is the eigen function of the one-electron Hamiltonian $H$ and $E_{k}(\vec{r})$ represents the eigen energy. $\vec{R}$ is the direct lattice vector. $U(\vec{r})$ is the crystalline periodic potential representing the influence of the crystal ions and electrons. 
After Bloch theorem, since $U(\vec{r})$ is a periodic potential, the solutions of Equation (1.4) are of the form of Bloch functions.

$$
\Psi_{n k}(\vec{r})=e^{i \vec{k} \vec{r}} U_{n k}(\vec{r})
$$

Where $U_{n k}(\vec{r})$ is periodic in the direct lattice and $n$ is the band index. From Bloch theorem again, one may show that the energy is periodic in the reciprocal lattice, that is, $E_{k+K}=E_{k}$. The problem can hence be reduced to a Brillouin zone cell defined previously. The main consequence of this periodicity is that all semiconductors feature a band gap, i.e. a region in which there are no allowed energy states. In turn, the allowed energy levels form bands. Upper bands are called conduction band while lower band are called valence bands. Electrons lying in the valence band do not participate in the conduction process. The parameter $E_{g}$ is called the band gap and corresponds to the energy required for an electron to transit from the valence band to the conduction band where it can contribute to the conduction process. In a metal, the conduction and valence band overlap, meaning that they have high conductivity. Additionally, what separates a semiconductor from an insulator is the size of the gap. Since the gap is taller in an insulator than in a semiconductor, a huge amount energy is necessary for an electron to transit from the valence band to the conduction band, which explains their high resistivity to the current conduction process. Figure 1-4 depicts the simplified band structures of semiconductors, insulators and metals.

(a)

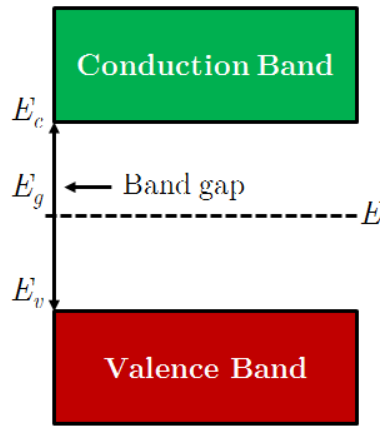

(b)

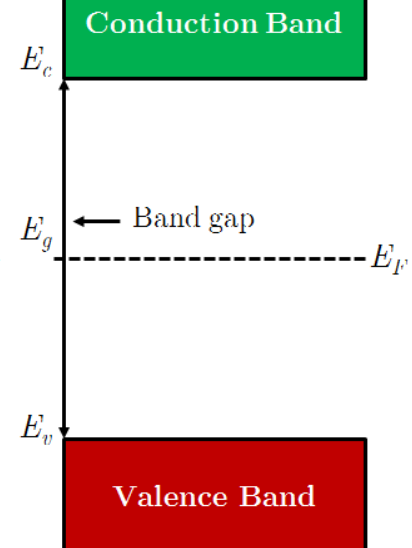

(c)

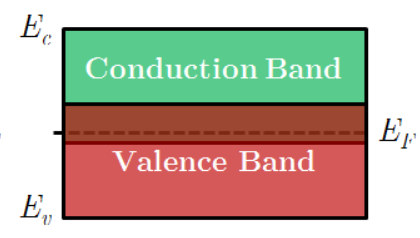
Figure 1-4: Simplified band structure of (a) semiconductors (b) Insulators and (c) Metals.
$E_{c}$ denotes the bottom of the conduction band, $E_{v}$ the top of the valence band and $E_{F}$ the Fermi level. The Fermi level is defined as the highest occupied energy state at $\mathrm{T}=0 \mathrm{~K}$. 
The band structure of semiconductors, i.e. the energy versus momentum may be approximated by parabolic functions:

In the conduction band:

$$
E=E_{g}+\frac{\hbar^{2} k^{2}}{2 m_{e}}
$$

In the valence band:

$$
E=-\frac{\hbar^{2} k^{2}}{2 m_{h}}
$$

Where $E g$ is the band gap, $\hbar$ is the Planck constant, $k$ is the wave vector, $m_{e}$ and $m_{h}$ are the free electron and hole effective masses, respectively.

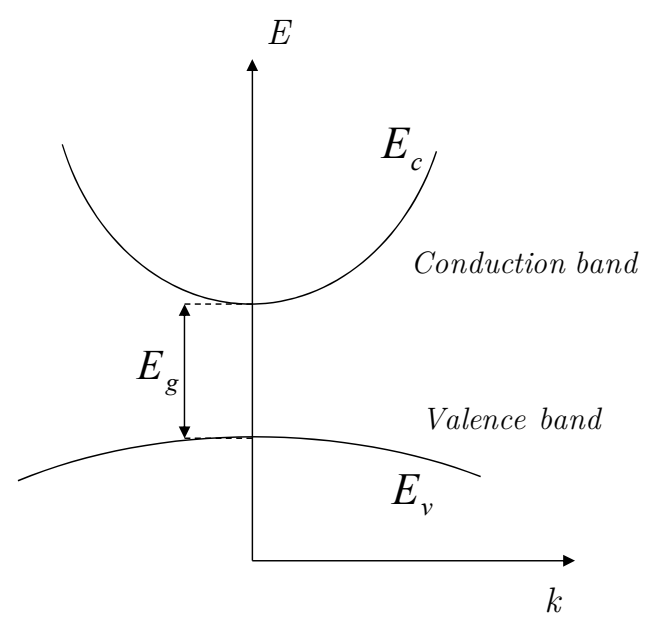

Figure 1-5: Band structure of an intrinsic semiconductor in the k-space

The effective mass represents mathematically the curvature of the energy band of a semiconductor and is defined in terms of tensorial components by:

$$
\frac{1}{m_{i j}^{*}}=\frac{1}{\hbar^{2}} \frac{\partial^{2} E(k)}{\partial k_{i} \partial k_{j}}
$$

As can be observed in Equation (1.9), the greater the effective mass, the smaller the curvature of the band. Physically, charge carriers can reach a maximum velocity inversely proportional to the effective mass. As parabolic band structure is only an approximation, the real band structures of semiconductors are 
computed by means of specific numerical methods. Silicon band diagram is illustrated in Figure 1-6. It is worth noting that this material has an indirect band gap, which implies that if an electron lies in the conduction band, it will require some change of momentum $\Delta k$ to gain the valence band. This change of momentum can be produced by a phonon, i.e. a vibration of the lattice, but has very weak probability to occur simultaneously with an electron transition from the conduction band to the valence band. Therefore, since the electron cannot rejoin the valence band by radiative recombination (i.e. emission of a photon by electron-hole pair recombination process), conduction band electrons typically last quite some time before recombining through less efficient means. As a result, silicon is naturally not efficient for light-emitting or laser diodes.

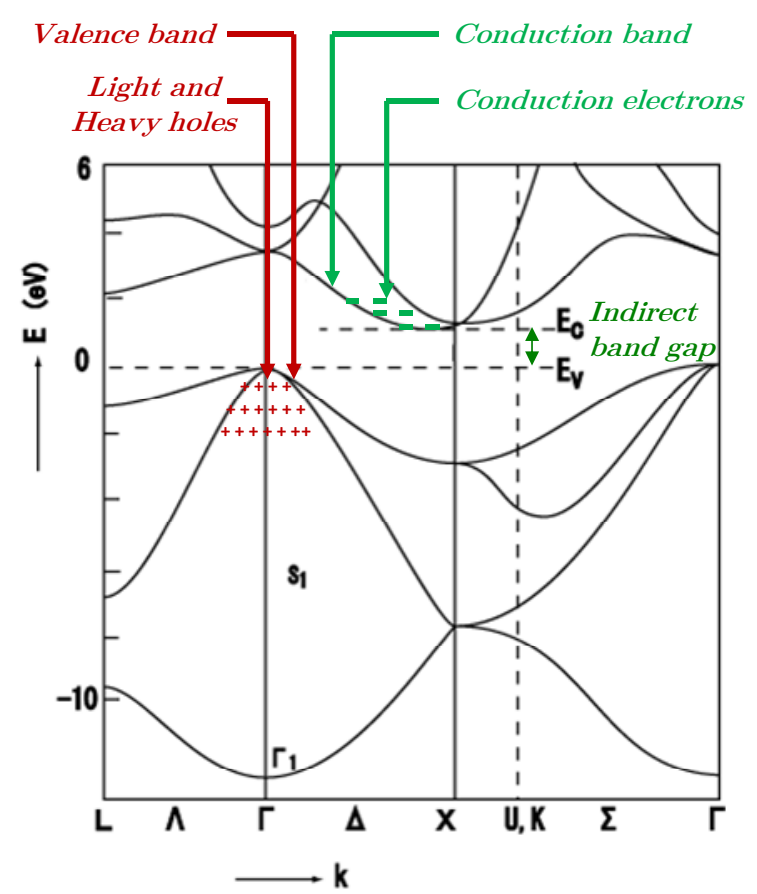

Figure 1-6: Calculated silicon band structure. The valence band is split in two bands with wider/narrower curvature corresponding to heavy/light holes, respectively (blues crosses). The conduction electrons lie in the conduction band (red minuses). (After [10])

\subsubsection{Doping silicon}

Silicon is located in the fourth (IV) columns of the periodic table of elements, which means that it has four bonding (Valence) electrons making it easy to combine with other elements. Usually found amorphous in nature, silicon can crystallize with high purity in a diamond cubic structure as a result of well established technological processes. Doping silicon is the process of introducing 
impurities from the third (III) of fifth (IV) columns of the periodic table of elements in order to modify and enhance its intrinsic electrical properties. Elements from the third (fifth) columns have three (five) bounding electrons, respectively. As they are introduced into the silicon crystal, and substitute some individual silicon atoms, III-V elements introduce either a lack or an excess of one electron per atom, and hence are called "acceptor" or "donor" (of electrons), respectively. The resultant doped semiconductor is denominated "p-type" (positive) or " $n$-type" (negative), respectively (Figure 1-7) ("Intrinsic" means that the semiconductor is left undoped).

(a)

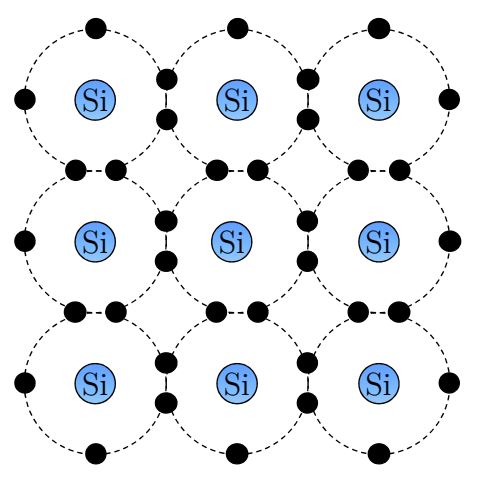

Intrinsic (b)

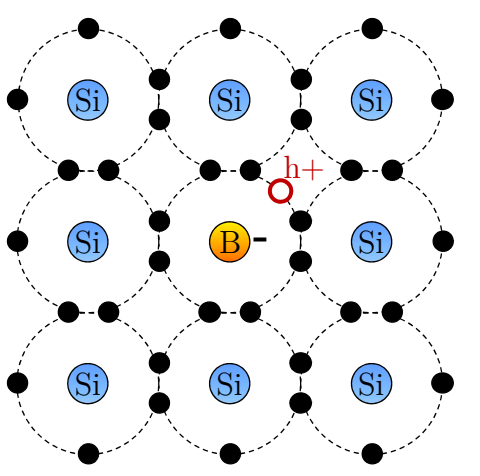

P-type (c)

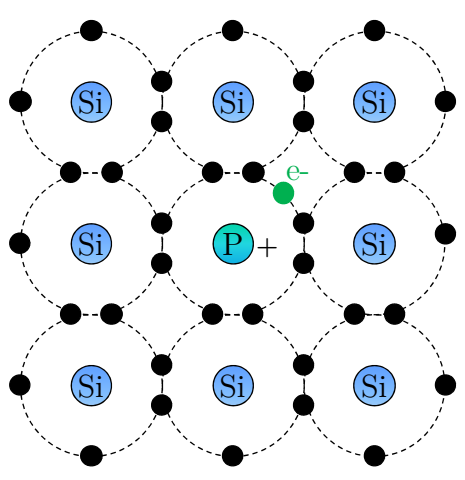

N-type

Figure 1-7: Schematic representation of (a) intrinsic silicon, (b) p-type silicon with acceptor impurity (boron) and (c) n-type silicon with acceptor impurity (Phosphorus)

The introduction of impurities creates new energy levels called donor $E_{d}$ and acceptor $E_{a}$ levels corresponding to respectively to $n^{-}$and $p$-type semiconductor, as shown in Figure 1-8.

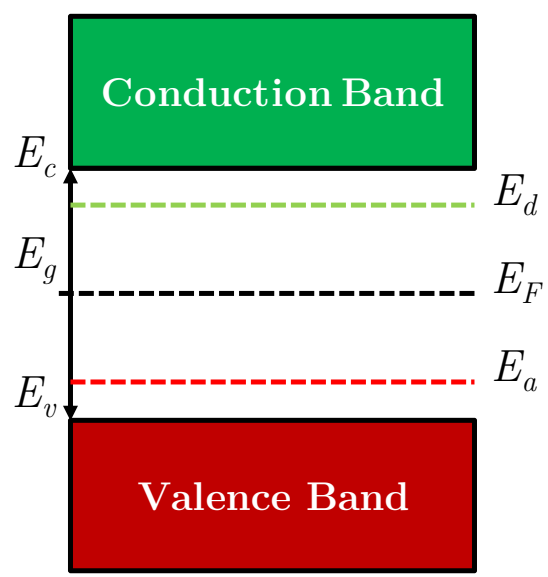

Figure 1-8: Schematic of a band structure with donor and acceptor energy levels 
The excess or lacking electrons (holes) can move almost "freely" through the crystal (as they are weakly bond to the crystal) in the form of a free carrier electrical current. The free electron and hole motions can be controlled through the realization of junctions between $p$ and $n$-type semiconductors. A variety of junctions exists and exhibit different electrical response. Here, we are interested in one very common type of semiconductor junction: the $p n$ diode.

\section{2 pn Junction Theory}

\subsubsection{Forming a pn junction in silicon}

A $p n$ junction is formed by combining together two $p$ and $n$-type semiconductors. The resultant boundary can be ideally thought as a straight non-conductive volume called the depletion region as depicted in Figure 1-9. The physics of abrupt junctions was first described after [11] Shockley's ideal model, which will be developed in the following.

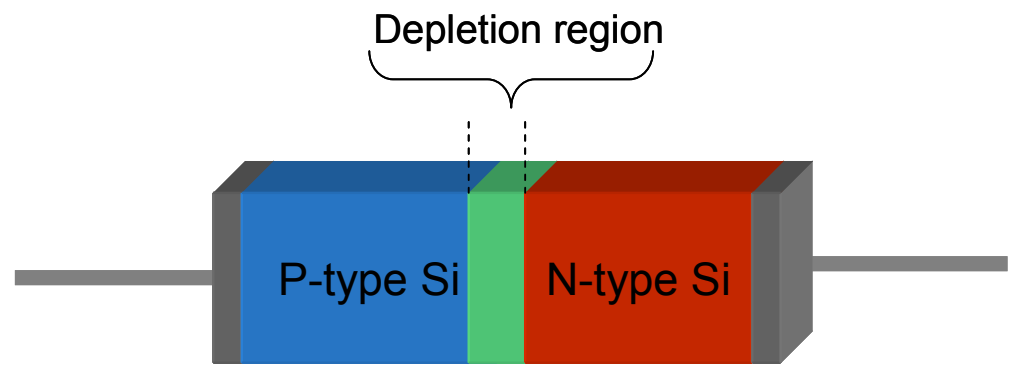

Figure 1-9: schematic representation of an ideal abrupt silicon $p n$ junction

\subsection{2 pn Junction in equilibrium state}

Assuming an abrupt $p n$ junction (Figure 1-9), when both $p$ and $n$-type semiconductors are in close contact a concentration gradient appears, causing negative charges (electrons) to diffuse in the $p$-type region, and to leave positively charged ions (acceptors). Likewise, the positives charges (holes) tend to diffuse in the $n$-type region, leaving negatively charged ions (donors). This charge separation gives rise to an electric field preventing electrons and holes to diffuse further by tending to pull them back to their original positions (i.e. before forming the $p n$ junction at $t=0)$. As charge carriers experience counteracting forces, they reach a steady state, leaving an isolating layer refers to as depletion region (or space charge region) because it is depleted of charge carriers (Figure 
1-10). Thus, the junction is at equilibrium under a potential difference designated built-in-potential $V_{b i}$, which can be derived from Figure 1-11 as:

$$
q V_{b i}=q V_{p}+q V_{n}=\left(E_{i}-E_{F}\right)_{p . s i d e}+\left(E_{F}-E_{i}\right)_{n . s i d e}
$$

Where $E_{F}$ and $E_{i}$ are the real and the intrinsic Fermi level of the semiconductor.

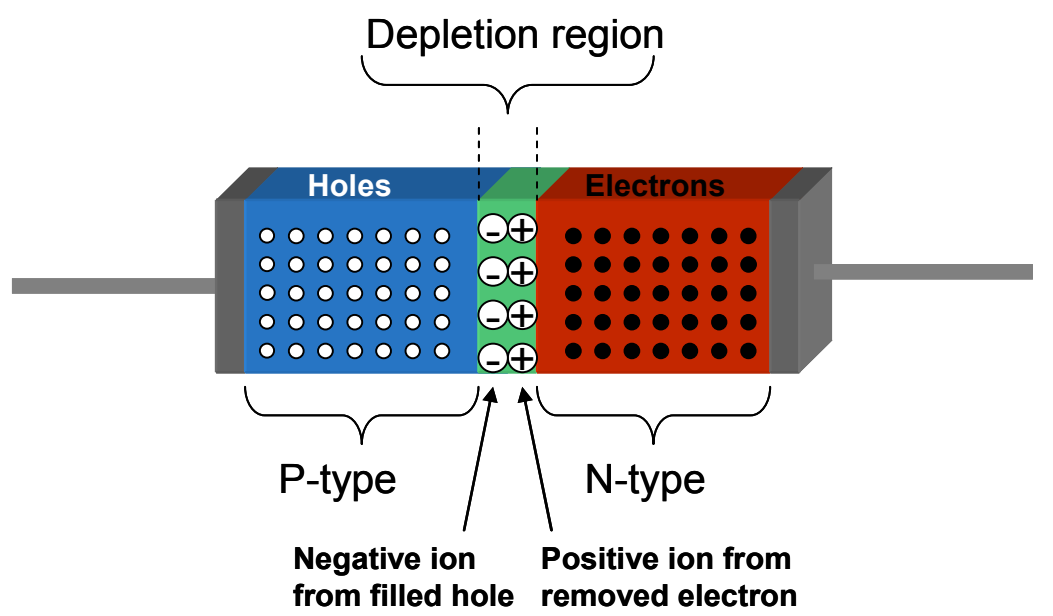

Figure 1-10: $p n$ junction at equilibrium

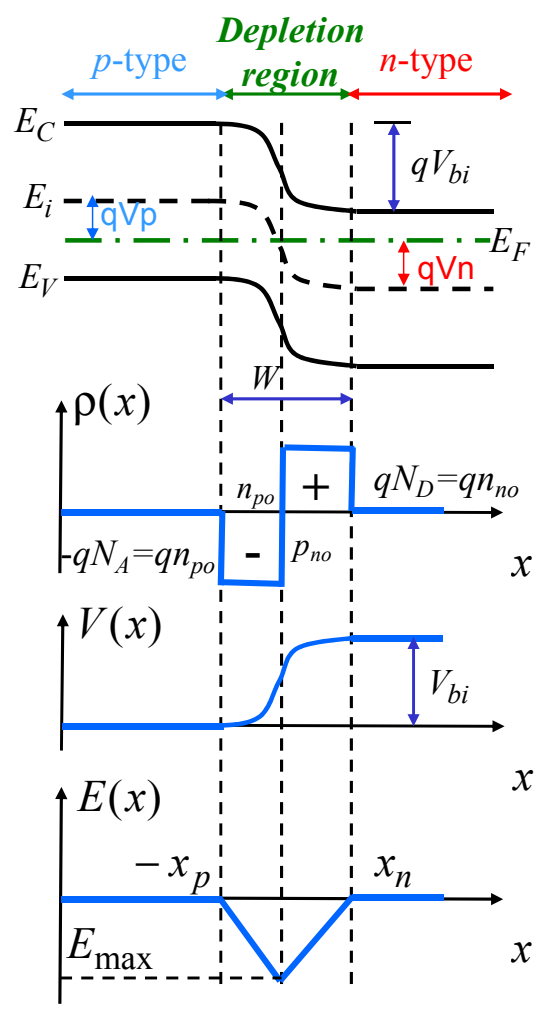

(a)

(b)

Figure 1-11: Abrupt pn junction at thermal equilibrium (a) energy band diagram. (b) space-charge distribution. (c) Potential as a function of the distance. $V_{b i}$ is the built-in potential, (d) Electric field distribution 
The majority carrier concentrations, i.e. electrons in the $n$-type region $n_{n_{0}}$, and holes in the $p$-type region $p_{p_{0}}$ are given by:

$$
\begin{aligned}
& n_{n_{0}}=n_{i} \exp \left(\frac{\left(E_{F}-E_{i}\right)_{n . s i d e}}{k_{B} T}\right) \simeq N_{D} \\
& p_{p_{0}}=n_{i} \exp \left(\frac{\left(E_{i}-E_{F}\right)_{p . s i d e}}{k_{B} T}\right) \simeq N_{A}
\end{aligned}
$$

Where $N_{A}$ and $N_{D}$ are the concentration of donor and acceptors, respectively. Hence from $(1.10),(1.11)$ and (1.12), the built-in potential can be calculated as:

$$
V_{b i}=\frac{k_{B} T}{q} \ln \left(\frac{p_{p_{0}} n_{n_{0}}}{n_{i}^{2}}\right) \simeq \frac{k_{B} T}{q} \ln \left(\frac{N_{A} N_{D}}{n_{i}^{2}}\right)
$$

Using 1D Poisson's Equation:

$$
\frac{\partial^{2} V}{\partial x^{2}}=\frac{\rho(x)}{\mathcal{E}_{r} \mathcal{E}_{0}}
$$

One can derive the depletion width for a symmetric two sided abrupt junction:

$$
W=\sqrt{\frac{2 \varepsilon_{r} \varepsilon_{0}\left(N_{A}+N_{D}\right) V_{b i}}{q N_{A} N_{D}}}
$$

For a one-sided abrupt junction $\left(p^{+} n\right.$ or $\left.p n^{+}\right) \quad N_{B}=N_{D}$ or $N_{A}$ depending on whether $N_{D}>>N_{A}$ or vice versa:

$$
W=\sqrt{\frac{2 \varepsilon_{r} \varepsilon_{0}\left(V_{b i} \mp V\right)}{q N_{B}}}
$$

The depletion layer capacitance for a one sided abrupt junction is given by: 


$$
C=\frac{d Q_{c}}{d V}=\frac{q N_{B} d W}{d V}=\sqrt{\frac{q N_{B} \mathcal{E}_{r} \mathcal{E}_{0}}{2\left(V_{b i} \mp V\right)}}
$$

\subsection{3 pn junction under an applied electric field}

Depending upon the voltage applied across the cathode and anode (forward or reverse bias) the junction does not behave the same way. Here, we describe the charge carrier motion under an external electric field and derive the ideal Shockley's current-voltage characteristic of a $p n$ junction. The description of charge carrier behavior from to Shockley's Equation is valid under the following assumptions:

1. The depletion layer is abrupt.

2. The density of injected minority carrier density is much smaller than the majority carrier density.

3. There is no generation current in the depletion layer and electron and hole currents are constant through the depletion layer.

Using continuity Equation, the minority carrier densities in the quasi neutral region are given by:

$$
\begin{aligned}
& \Delta n_{p}(x)=n_{p}-n_{p_{0}}=n_{p_{0}}\left(e^{q V / k T}-1\right) e^{\left(x-x_{n}\right) / L_{p}} \\
& \Delta p_{n}(x)=p_{n}-p_{n 0}=p_{n 0}\left(e^{q V / k T}-1\right) e^{-\left(x-x_{n}\right) / L_{p}}
\end{aligned}
$$

Where $L_{n}=\sqrt{D_{n} \tau_{n}}$ and $L_{p}=\sqrt{D_{p} \tau_{p}}$ are the diffusion lengths of minority carriers, i.e. the average distance electrons and holes travel into the $p$ and $n$-type regions, respectively without recombining with a particle of the opposite charge or being trapped by lattice defects. $D_{n}=\mu_{n} k T / q, \quad D_{p}=\mu_{p} k T / q$ are the diffusion coefficients (Einstein relationships) and $\mu_{n}, \mu_{p} \tau_{n}$ and $\tau_{p}$ are respectively the mobilities and lifetime of electrons and holes. The lifetime is basically the time between generation and recombination of electrons and holes caused by either electron-hole recombination or charge carrier trapping. Equations (1.18) and (1.19) are represented respectively in Figure 1-12 and Figure 1-13, showing the motion of charge carriers across a $p n$ junction polarized under either forward or reverse bias. 
Forward bias: When a forward bias is applied across the junction, i.e. the $p$ type region is connected to the positive terminal (cathode) and the $n$-type region is connected to the negative terminal (anode) the electron and holes are pushed towards the junction (Figure 1-12). As a result, the densities of minority carriers (holes in the $n$-type region, electrons in the $p$-type region) increase in the quasi neutral region. With increasing forward-bias voltage, the depletion zone eventually becomes thin enough and the potential barrier is lowered so that the space charge region electric field can no longer prevent charge carrier motion across the $p n$ junction, hence reducing the electrical resistance. Electrical current starts flowing at a given threshold voltage.

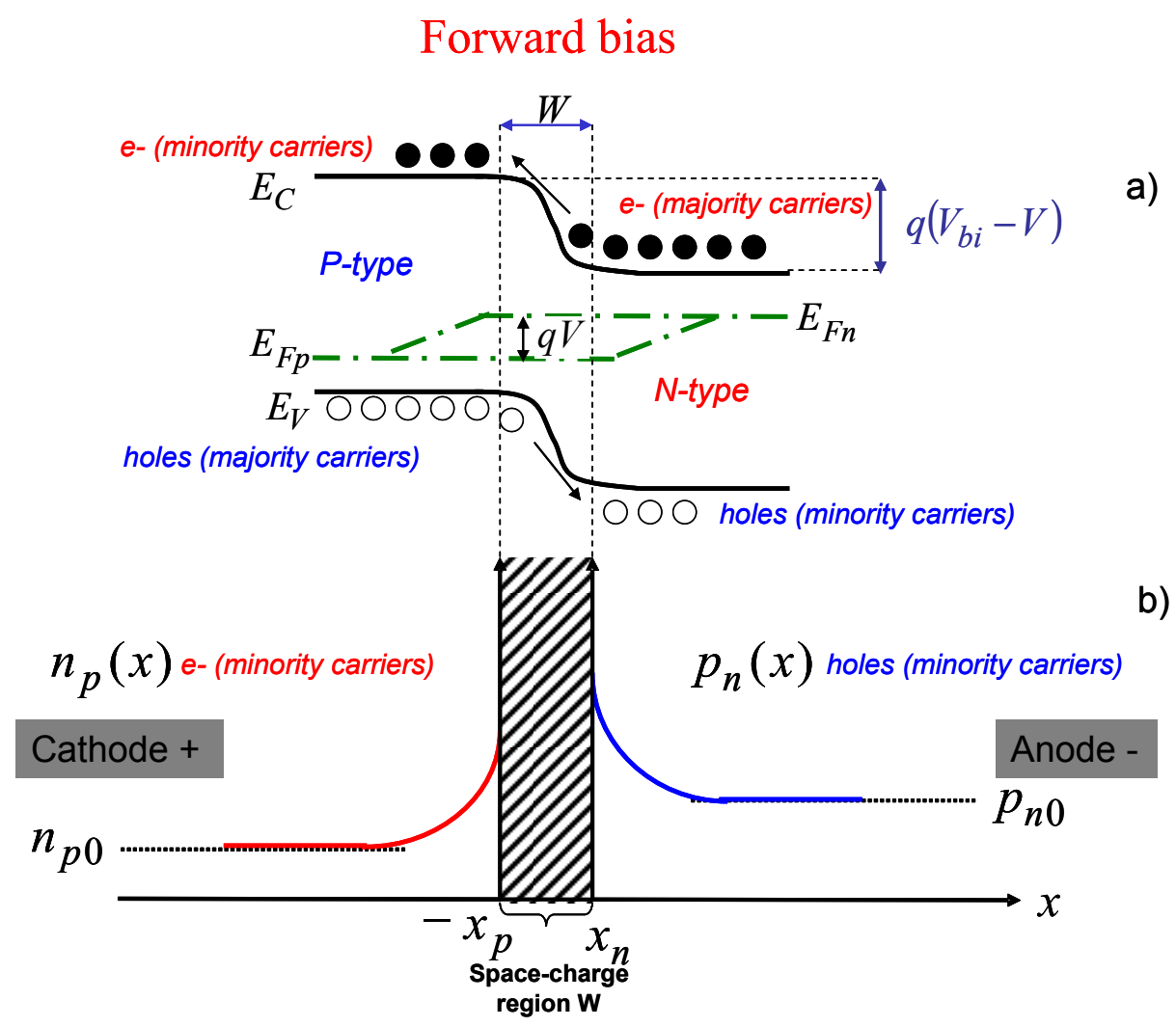

Figure 1-12: (a) Energy band diagram under forward bias. (b) Carrier distribution under forward bias

Reverse bias: When a reverse bias is applied, i.e. the $p$-type region is connected to the negative terminal (cathode) and the $n$-type region is connected to the positive terminal (anode), electrons and holes are pulled away from the junction thus increasing the size of the depletion region (Figure 1-13). Increasing further the reverse-bias voltage causes the space charge region to widen and the potential barrier to be taller, which prevent charge carrier motion from being triggered 
across the junction. The junction is depleted of charge carriers. As a result, very little reverse saturation current flows due to minority carriers being collected on the order of the diffusion length.

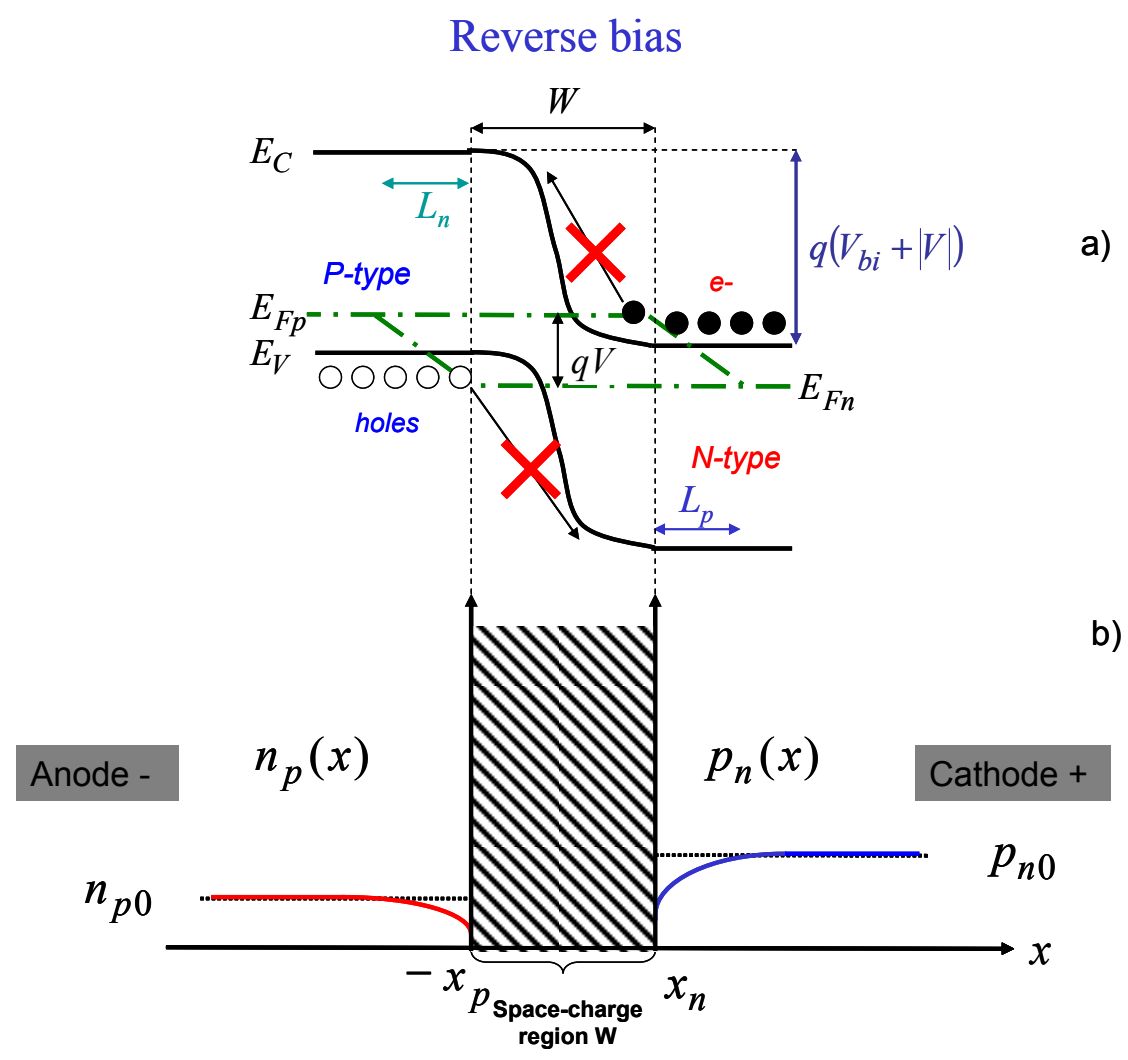

Figure 1-13: (a) Energy band diagram under reverse bias. (b) Carrier distributions under reverse bias

\subsubsection{Ideal current-voltage characteristics: Shockley's equation}

Shockley' Equation describes an ideal diode behavior under an external applied voltage. This ideal current-voltage characteristic is derivated from the basic current-density Equations:

$$
\begin{aligned}
& J_{n}=J_{n}^{\text {drift }}+J_{n}^{\text {diff }}=q \mu_{n} n \xi+q D_{n} \nabla n \\
& J_{p}=J_{p}^{\text {drift }}+J_{p}^{\text {diff }}=q \mu_{p} p \xi+q D_{p} \nabla p \\
& J_{\text {cond }}=J_{n}+J_{p}
\end{aligned}
$$

$D_{n}=\mu_{n} k T / q, D_{p}=\mu_{p} k T / q$ are the diffusion coefficients (Einstein relationships), $\mu_{n}, \mu_{p}, n, p, \nabla n, \nabla p$ are respectively the mobilities, concentrations and gradients of concentration of electrons and holes. $J_{n}$ an $J_{\mathrm{p}}$ are respectively the electron and hole current densities, which consist of the contribution of two terms: the drift 
and diffusion components respectively caused by the field and the carrier concentration gradient. $J_{\text {cond }}$ is the conduction current density summing the contribution of electron and hole current densities. From Equations (1.18), (1.19), and $(1.20),(1.21)$ one can derive the minority carrier diffusion current densities (Figure 1-14). In this case, The drift current contribution is negligible, i.e. $J_{p}^{\text {drift }} \ll J_{p}^{\text {diff }}$, hence it follows:

$$
\begin{aligned}
& J_{n}^{\text {diff }}=-q D_{n} \frac{\partial n_{p}}{\partial x}=\frac{q D_{n} n_{p 0}}{L_{p}}\left(e^{q V / k T}-1\right) e^{\left(x+x_{p}\right) / L_{n}} \\
& J_{p}^{\text {diff }}=-q D_{p} \frac{\partial p_{n}}{\partial x}=\frac{q D_{p} p_{n 0}}{L_{p}}\left(e^{q V / k T}-1\right) e^{-\left(x-x_{n}\right) / L_{p}} \\
& J_{\text {tot }}=J_{n}^{\text {diff }}+J_{p}^{\text {diff }}
\end{aligned}
$$

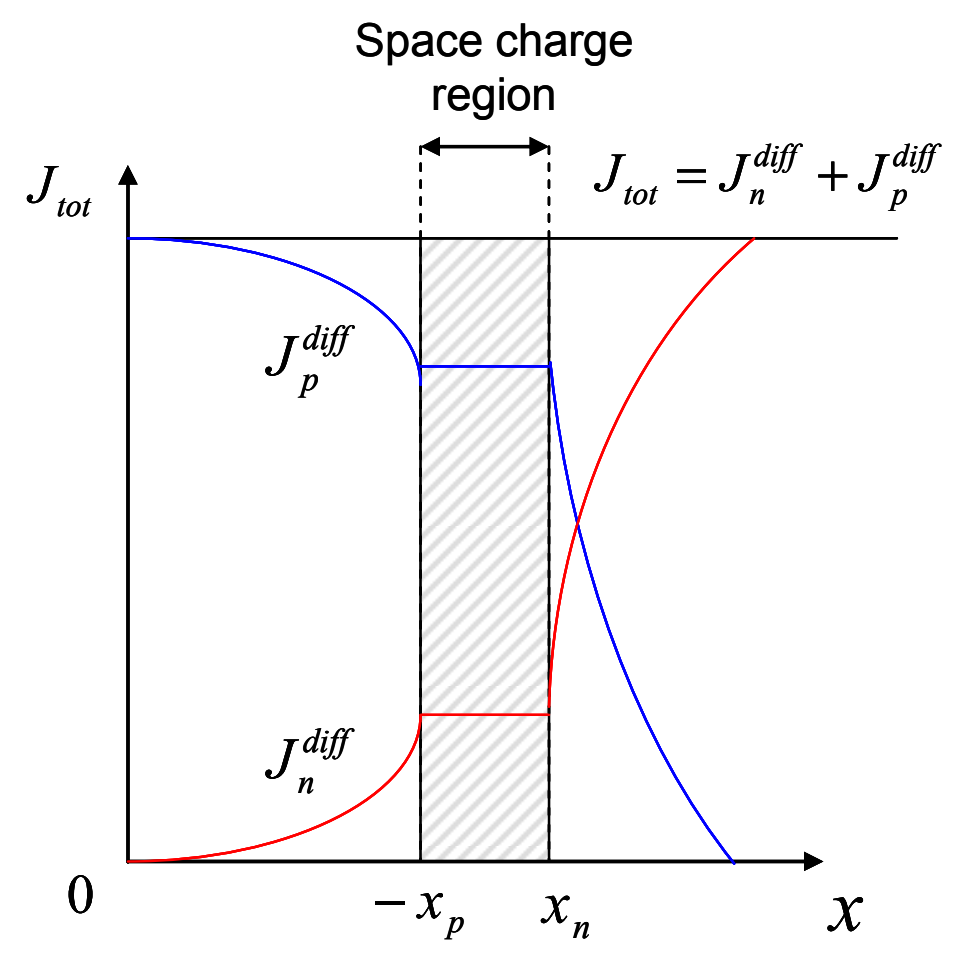

Figure 1-14: Current densities in a PN junction

At the edges of the space charge region $\left(-x_{p}, x_{n}\right)$ assuming that there is no electron-hole generation-recombination processes taking place, the total current is then the sum of the minority carrier diffusion current: 


$$
J_{\text {tot }}=J_{n}^{\text {diff }}||_{x_{p}}+\left.J_{p}^{\text {diff }}\right|_{x_{n}}=J_{s}\left(e^{q V / k T}-1\right)
$$

Where $J_{s}=\frac{q D_{p} p_{n 0}}{L_{p}}+\frac{q D_{n} n_{p 0}}{L_{n}}$.

Equation (1.26) is the Shockley's mathematical relationship describing the current dependence of an ideal pn diode as a function of the voltage applied. Figure 1-15 shows the diode operation under forward and reverse bias.

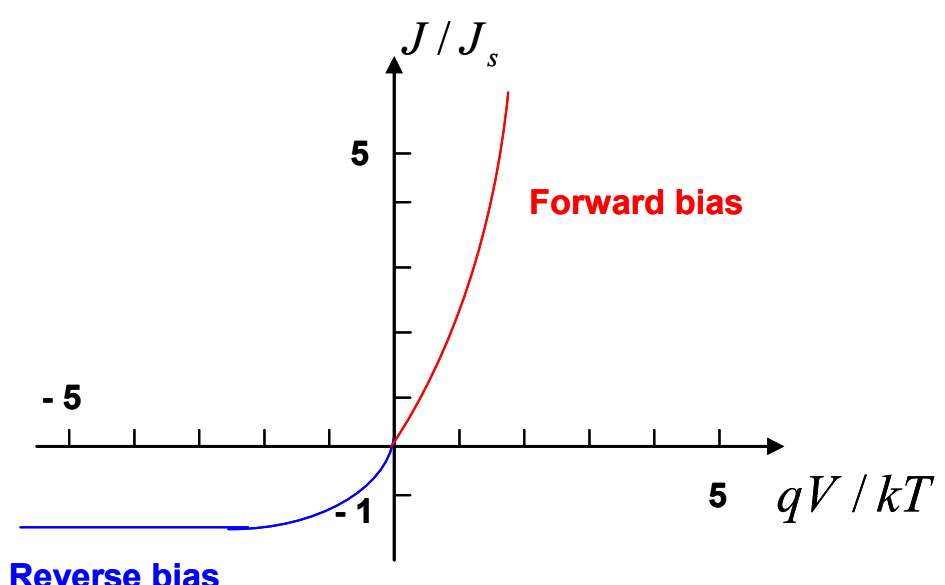

Figure 1-15: Current voltage characteristics of an ideal $p n$ junction

As can be observed in Figure 1-15 the diode response depends on the sign of the applied voltage. In reverse bias, very little current flows through the junction. This is called the reverse saturation current, $J_{s}$. In forward bias the current increases exponentially. Shockley's Equation well describes the current-voltage characteristics of Germanium $p n$ junctions for low density currents. However, for $\mathrm{Si}$ and GaAs pn junctions, more complex modeling is required as Shockley's characteristic provides only a qualitative description. Generation and recombination of carriers in the depletion region, surface effects, tunneling of carriers between states within the band gap, have to be included. For the sake of completeness, Figure 1-16 shows the real current-voltage characteristics of a practical Si diode. 


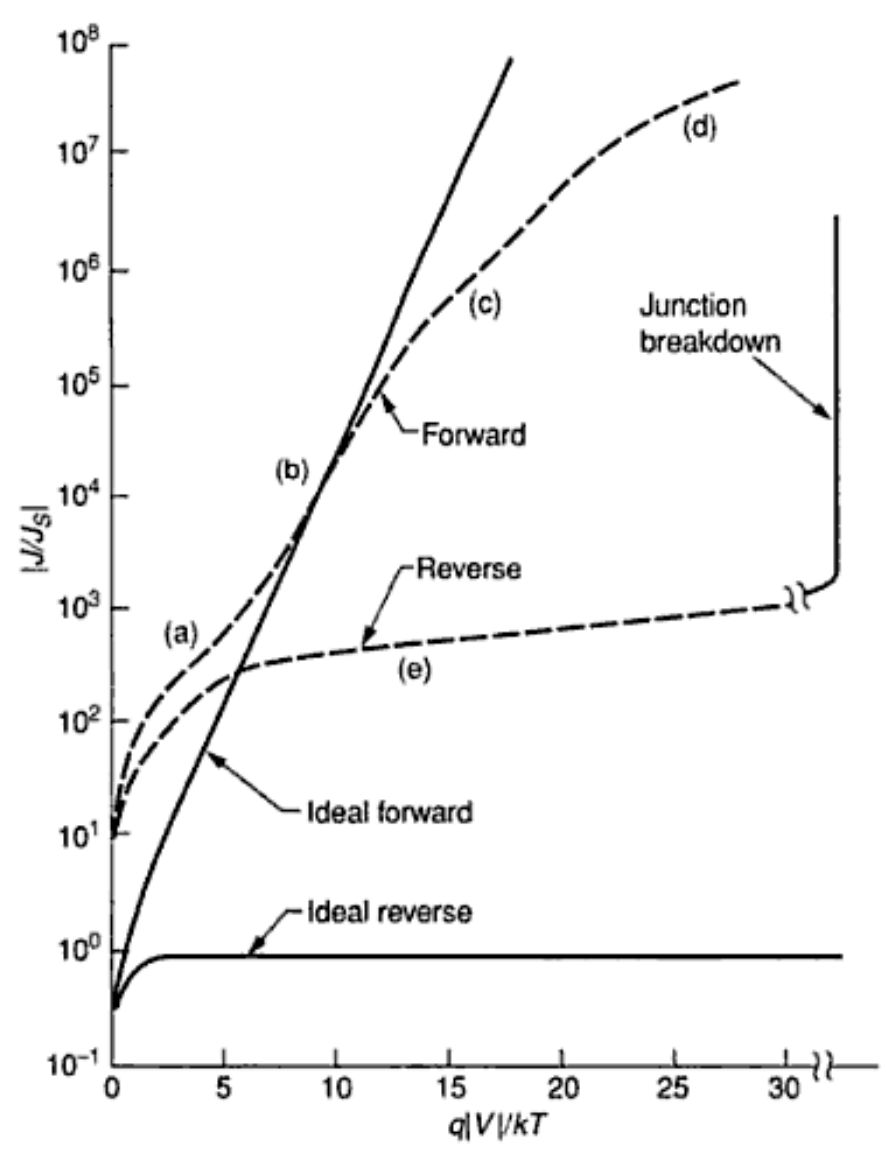

Figure 1-16: Current voltage characteristics of a real $\mathrm{Si}$ diode (a) generationrecombination current region. (b) Diffusion current region. (c) High-injection region. (d) Series resistance effect. (e) Reverse leakage current due to generation-recombination and surface effects. Picture taken from reference [12]

\subsubsection{Fabrication process of pn junctions}

In practice, a $p n$ junction is made either by diffusion, implantation or molecular beam epitaxy (MBE). In the case of diffusion, the dopants diffuse in under heating so that the surface acceptor concentration exceeds the donor concentration. Implantation enables to make sharper junctions by bombarding the crystal with ion species from the III or $\mathrm{V}$ column of the periodic table of the elements. The drawback of this method is the damage caused to the crystal due to implanted impurities. Further annealing is generally required for the ions to redistribute uniformly within the crystal (denominated dopant activation) and repair the crystal dislocations due to high energy ions impinging on the semiconductor surface. MBE may be useful to make horizontal $p n$ junction, or stacked doped layers, for instance. 


\subsection{Fundamentals of Light Propagation in Optical Waveguides}

\subsubsection{Ray optics approach}

Assuming two isotropic and homogeneous media with respective refractive indices $n_{1}$ and $n_{2}$, a light ray propagating from one medium to the other is refracted according to Snell's-Descartes's law. The relationship between the angles formed the incident and refracted ray is given by.

$$
n_{1} \sin \left(\theta_{1}\right)=n_{2} \sin \left(\theta_{2}\right)
$$

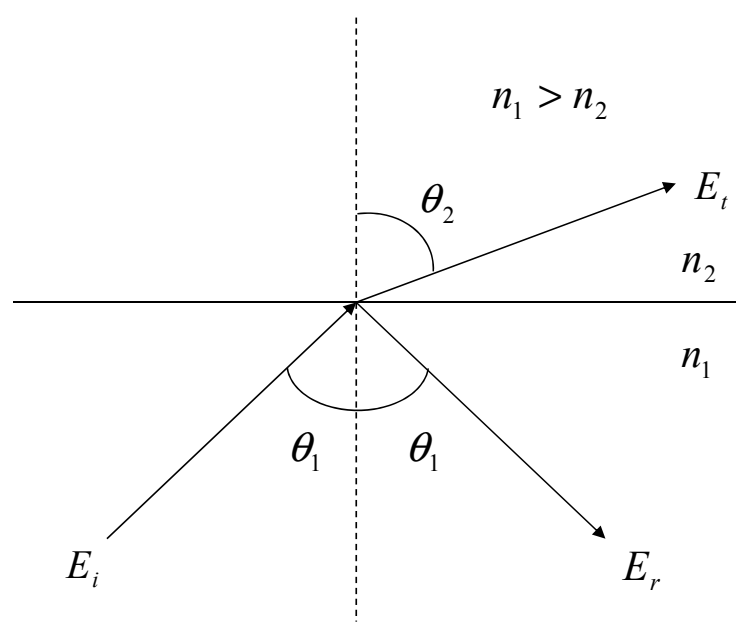

Figure 1-17: Snell's-Descartes Law

If we now consider a $2 \mathrm{D}$ slab waveguide, i.e. infinite in the $\mathrm{x}$-dimension, as depicted in Figure 1-18, the angle of incidence is such that the incident ray is not refracted but reflected back into the medium with refractive index $n_{1}$. This is the case when $\theta_{1}>\theta_{c}$, where the critical angle $\theta_{c}$ is given by:

$$
\theta_{2}=90^{\circ} \Rightarrow \sin \theta_{c}=\frac{n_{1}}{n_{2}}
$$

In that case the confined rays follow a zig-zag path and are hence guided within the central layer of the waveguide by TIR. 


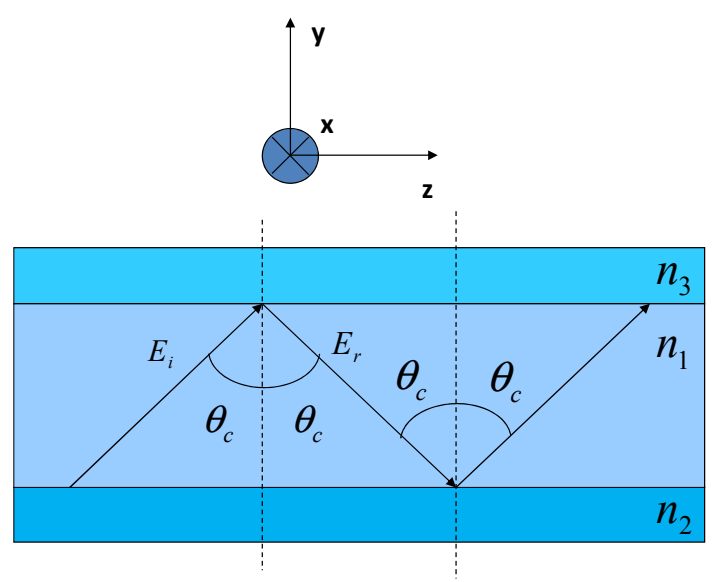

Figure 1-18: Light guidance by total internal reflection in a 2D slab waveguide along the z-axis

Even though the condition $\theta_{1} \geq \theta_{c}$ is satisfied, only a discrete set of rays with certain angles can be guided. These discrete sets of guided rays are called modes of the slab waveguide. In addition, the guidance of optical rays depends on their polarization. One can distinguish between two polarizations in slab waveguides: under pure TE (TM) polarization, the electric (magnetic field) is perpendicular to the plane of incidence (the plan defined by the incident, refracted and reflected rays, see Figure 1-19).

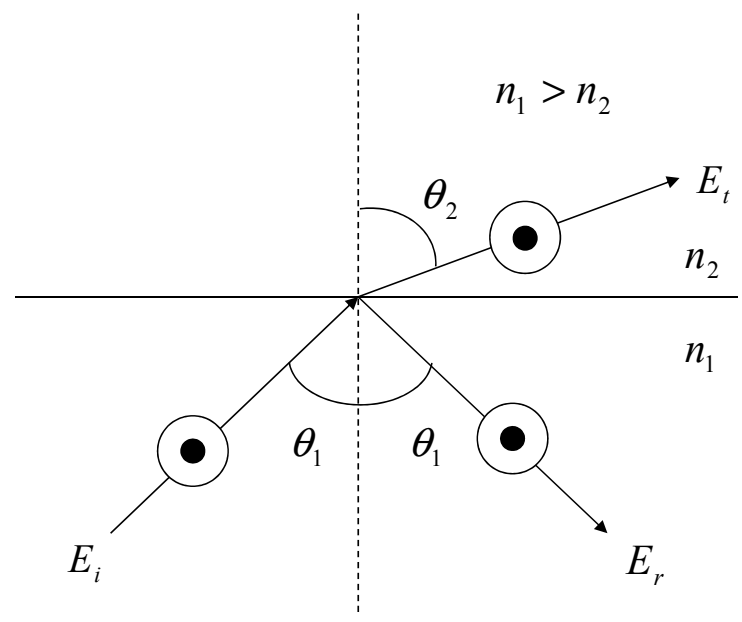

Figure 1-19: TE (TM) polarized rays: the electric (magnetic) field is perpendicular to plane of incidence.

\subsubsection{Extension of the ray optics approach}

Now considering that the rays are propagating waves with a given wave vector $k$, one can define the components: 


$$
\begin{aligned}
& k_{z}=n_{1} k_{0} \sin \theta_{1} \\
& k_{y}=n_{1} k_{0} \cos \theta_{1}
\end{aligned}
$$

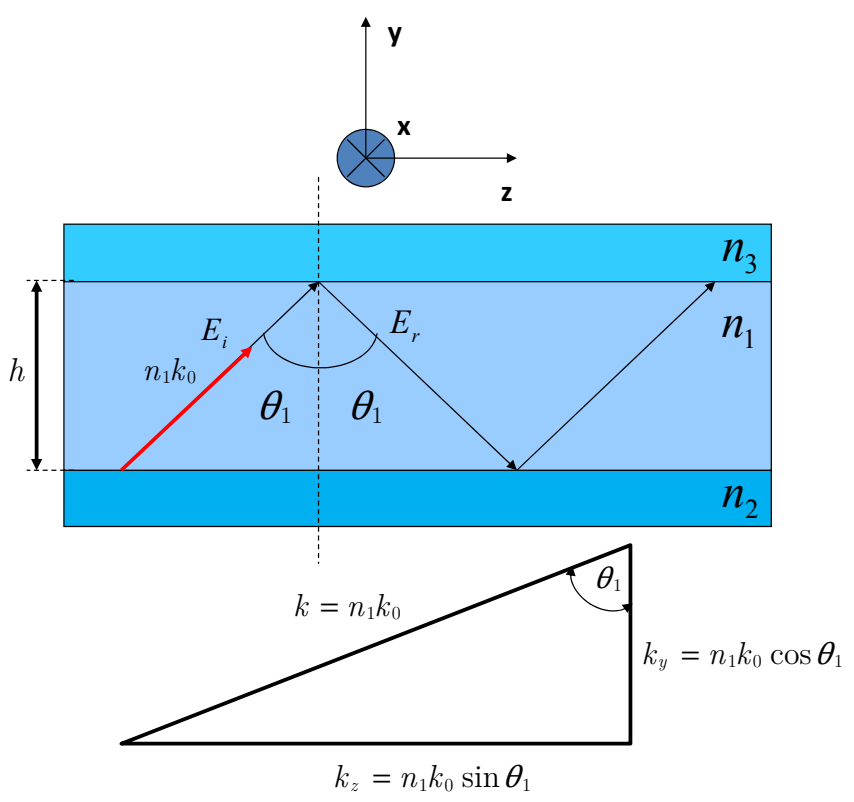

Figure 1-20: Extension of the ray optics approach associating a propagating wave following the paths traced by the ray

The transversal (y axis) phase shift experienced by the wave due to the zigzag path in a round trip is given by:

$$
\varphi=2 k_{y} h=2 k_{0} n_{1} h \cos \theta_{1}
$$

Moreover, the phase shifts experienced by the ray due to total internal reflection at the upper $\left(\varphi_{c}\right)$ and lower-boundaries $\left(\varphi_{s}\right)$ are given for $\mathrm{TM}$ and $\mathrm{TE}$ polarizations respectively by:

$$
\begin{gathered}
\boldsymbol{\varphi}_{c}=2 \tan ^{-1} \frac{\sqrt{\left(\frac{n_{2}{ }^{2}}{n_{1}}\right) \sin ^{2} \theta_{1}-1}}{\frac{n_{2}}{n_{1}} \cos \theta_{1}} \\
\varphi_{s}=2 \tan ^{-1} \frac{\sqrt{\sin ^{2} \theta_{1}-\left(\frac{n_{2}{ }^{2}}{n_{1}}\right)}}{\cos \theta_{1}}
\end{gathered}
$$


And the self consistency condition for constructive interference (and therefore light guidance) implies that the total phase shift must be zero or a multiple of $2 \pi$ hence:

$$
2 k_{0} n_{1} h \cos \theta_{m}-\varphi_{c}-\varphi_{s}=2 \pi m
$$

Where $\mathrm{m}$ is a finite integer and corresponds to the mode order. Each mode has a unique propagation constant which component along the propagation direction is given by:

$$
\beta_{m}=k_{0} n_{1} \sin \theta_{m}
$$

Equation (1.35) shows the link that exists between the Ray optics model characterized by the propagation angle $\boldsymbol{\theta}_{m}$ and the electromagnetic wave treatment characterized itself by the propagation constant. Usually, the effective index of propagation is used to characterize the guided mode as follows:

$$
n_{e f f, m}=\frac{\beta_{m}}{k_{0}}=n_{1} \sin \theta_{m}
$$

And to achieve guidance:

$$
n_{2}, n_{3}<n_{e f f, m}<n_{1}
$$

Consequently, the greater the effective index, the stronger the confinement of the field inside the core is (with index $n_{1}$ ).

The ray optics approach and its extended version have given simultaneously an intuitive and quantitative picture optical propagation via TIR in optical waveguides. However, these models do not provide information on the field distribution and its related amplitude and intensity. A complete description of the mode profiles can be carried out solving Maxwell's Equations. 


\subsubsection{Electromagnetic approach}

Maxwell's Equations enable to make a rigorous study of the mode profiles propagating along the considered waveguide.

$$
\begin{aligned}
& \partial D=\rho \\
& \nabla \times E=-\frac{\partial B}{\partial t} \\
& \nabla B=0 \\
& \nabla H=J+\frac{\partial D}{\partial t}
\end{aligned}
$$

Where: $D=\varepsilon_{m} E, B=\mu_{m} H$

One can derive the wave equation that describes propagation of electromagnetic waves:

$$
\nabla^{2} E=\mu_{m} \varepsilon_{m} \frac{\partial^{2} E}{\partial t^{2}}
$$

For example, the solution for TE modes in an asymmetric planar waveguide will be derived solving the following simplified wave Equation. As the field is uniform and only exists in the $\mathrm{x}$ direction the wave Equation has the form:

$$
\frac{\partial^{2} E_{x}}{\partial y^{2}}+\frac{\partial^{2} E_{x}}{\partial z^{2}}=\mu_{m} \varepsilon_{m} \frac{\partial^{2} E_{x}}{\partial t^{2}}
$$

The field propagates along the z-axis and has the form:

$$
E_{x}=E_{x}(y) e^{-j \beta z} e^{j \omega t}
$$

Injecting (1.44) into (1.43) we obtain:

$$
\frac{\partial^{2} E_{x}}{\partial y^{2}}+k_{y i}^{2} E_{x}=0
$$


Where, $k_{y i}^{2}=k_{0}^{2} n_{i}^{2}-\beta^{2}, i=1,2,3 ;(i=1$ : waveguide core $; i=2$ : upper cladding; $i=3$ lower cladding).

Solving Equation (1.45) the modes in an asymmetrical planar waveguide are in the form:

Field profile in the upper cladding:

$$
E_{x}=E_{2} \exp \left[-k_{y 2}\left(y-\frac{h}{2}\right)\right] \quad \text { for } y \geq(h / 2)
$$

Field profile in the core:

$$
E_{x}=E_{1} \exp \left[-j k_{y 1} y\right] \quad \text { for }-(h / 2) \leq y \leq(h / 2)
$$

Field profile in the lower cladding:

$$
E_{x}=E_{3} \exp \left[-k_{y 3}\left(y+\frac{h}{2}\right)\right] \quad \text { for } y \leq-(h / 2)
$$

From the boundary conditions of the electric field at the interfaces, one obtains the following Equation (1.49) that allows us to calculate the $\beta$ parameter.

$$
\tan ^{-1}\left[\frac{k_{y 3}}{k_{y 1}}\right]+\tan ^{-1}\left[\frac{k_{y 2}}{k_{y 1}}\right]=k_{y c} h+m \pi \quad \text { for } y \geq(h / 2)
$$

\subsubsection{Conclusion}

In summary, we have exposed a short background on solid state physics together with an overview of the light propagation mechanisms in optical waveguides. The following chapter introduces the main parameters of electro-optical modulators as well as a description of the main physical mechanisms that may be used for optical modulation in silicon. 


\section{Chapter 2}

\section{Optical Modulators}

This chapter introduces the main parameters of electro-optical modulators followed by a description of the main physical mechanisms that may be used for optical modulation in silicon[13]. The state-of-the-art of silicon and silicon-based modulators is also provided. Additionally, the passive and active characterization techniques and setups are presented.

\subsection{What is an Optical Modulator?}

\subsubsection{Introduction and background}

An optical modulator is a device that modulates the primary properties (amplitude, phase, polarization...) of a coherent light waveform (carrier) propagating through free space or in an optical waveguide. The modulating signal may come in several forms such that modulation may be carried out either via electro-optical, magneto-optical, mechano-optical (see example in Figure 2-1) or acousto-optical effects. The prefix placed before "optical" defines the nature of the modulating signal. The modulating characteristics tells what device is useful for which specific applications such as displays, active Q switching, mode locking of lasers, optical metrology, telecommunications in general including optical fiber communications. Actually, today's long and medium range communication networks rely mainly on optical digital fiber links where encoding electrical signals into near-infrared modulated light is an essential function traditionally realized by discrete electro-optical modulators. 


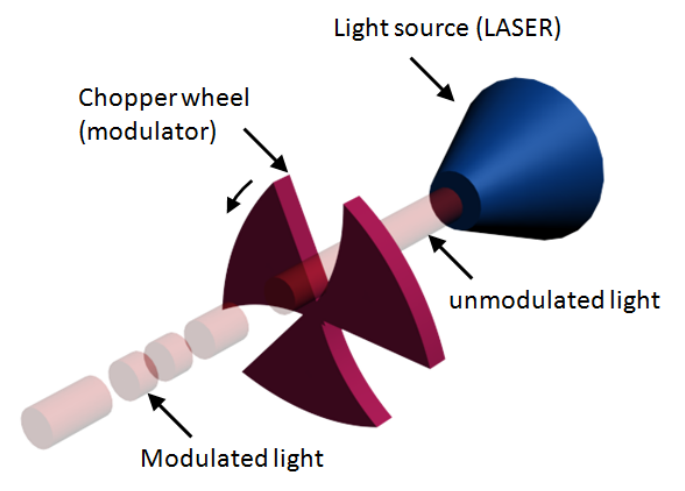

Figure 2-1: Basic working principle of a mechano-optical modulator

In contrast with analog optical modulation where the optical carrier is gradually modulated in phase or intensity via a continuous electrical signal, digital optical modulation converts a continuous light waveform (containing no information) into a discontinuous carrier that carries information in a binary form i.e. '1's and '0's referred to as 'bits'. These binary states correspond to when light is respectively switched on and off. Figure 2-2 illustrates an example of analog and digital amplitude modulations of a Mach-Zehnder interferometer (MZI) modulator.
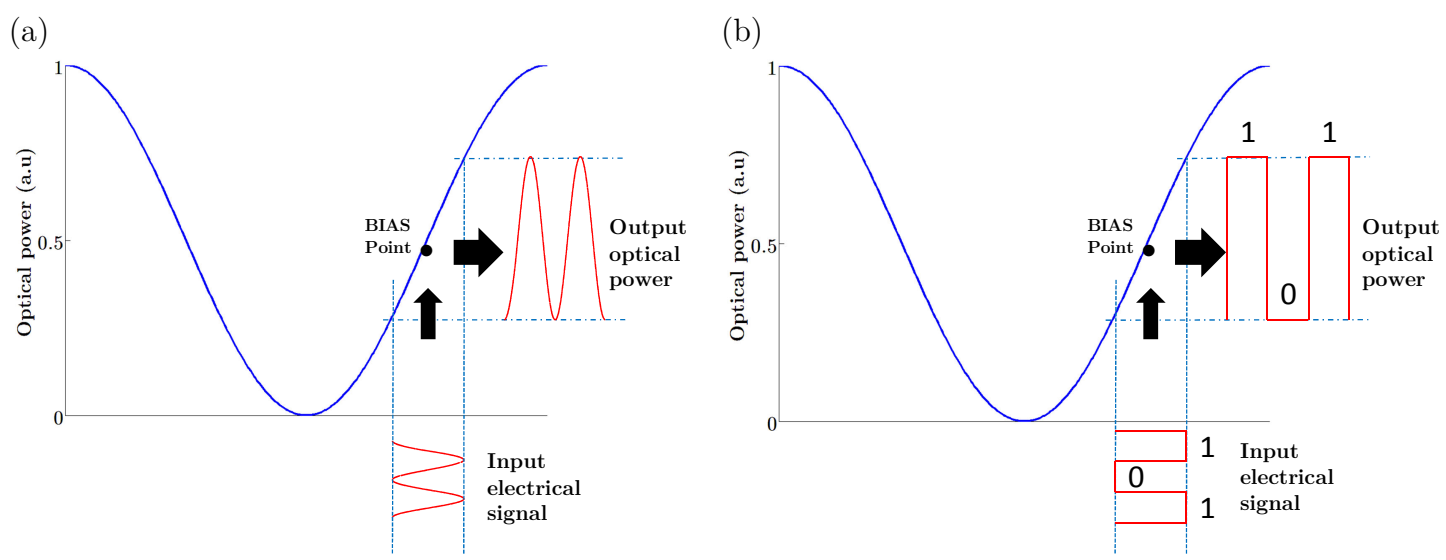

Figure 2-2: (a) Analog (b) digital amplitude modulation in a MZI modulator.

Existing commercial high speed modulators are essentially based on either electro-refraction or electro-absorption. Electro-refraction (respectively electroabsorption) is achieved via changing the real (resp. imaginary) part of the material's refractive index.

Common high speed transmission systems [14] include coherent light sources, typically vertical surface emitting lasers (VCSELs), Fabry-Pérot (FP) or distributed feedback lasers (DFP), which may be directly modulated. However, 
direct modulation is usually restricted to shorter links and lower speed $(\sim 2.5$ Gbits/s) due to the frequency alterations caused by the current supplied to the laser, which broadens the lasing peak and increase chromatic dispersion. For high data rates up to $40 \mathrm{Gbits} / \mathrm{s}$ and long haul transmissions, the lasers are operated in continuous wave and externally modulated by means of low chirp electroabsorption, or Mach-Zehnder modulators (MZM). The Internet originates from of such high speed modulation techniques and clearly surpasses copper cables for long and medium reach interconnects in term of bandwidth, loss and immunity to electromagnetic interferences. As a result, optical fibers and external lithium niobate $\left(\mathrm{LiNbO}_{3}\right)$ modulators coupled to a continuous wave $(\mathrm{CW})$ laser are the most successful combination for metro and long haul data transmission applications.

\subsubsection{Amplitude modulator main parameters}

Extinction ratio (ER): The extinction ratio of an amplitude modulator [14] is defined as the ratio of the output optical power in the "on" state $P_{o n}$ to the output optical $P_{\text {off }}$ in the "off" state (see Figure 2-3) and is given as:

$$
E R=10 \log _{10}\left(\frac{P_{o n}}{P_{\text {off }}}\right)
$$

Note that the DC ER or static extinction ratio is generally larger than the dynamic ER due to potential radio-frequency $(\mathrm{RF})$ losses at higher microwave frequencies and other RF performance issues.

Insertion loss (IL): The insertion loss is defined as the ratio between the optical input power $P_{i n}$ before entering the modulator and the "on" state as shown in Figure 2-3. Fiber-to-the chip coupling loss as well as passive component loss (taper, MMI, Y-junctions, curves...), which are technically not part of the device are excluded from the $P_{i n}$ value.

$$
I L=10 \log _{10}\left(\frac{P_{i n}}{P_{o n}}\right)
$$




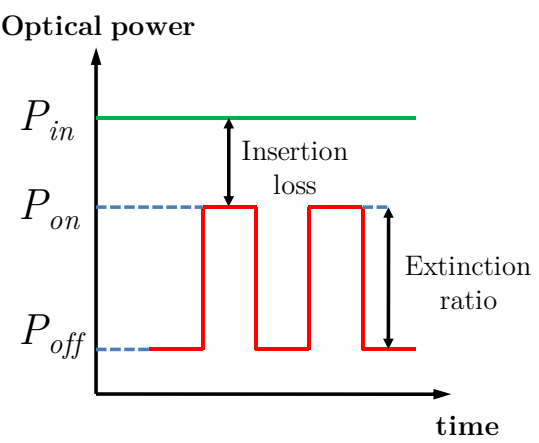

Figure 2-3: Graphical illustration of the extinction ratio and insertion loss

Drive voltage $\left(\boldsymbol{V}_{p p}\right)$ : The drive voltage is the voltage required by the modulator to achieve the desired level of performance in terms of ER. $V_{p p}$ denotes the peak-to-peak voltage. Low drive voltage are indeed highly desirable to reduce the overall power consumption (or switching energy), however the great majority of silicon optical modulators require relatively high drive voltages comprised between $2 \mathrm{~V}$ and $8 \mathrm{~V}$. It should be noticed that current $45 \mathrm{um}$ CMOS transistor swing voltage requirement is $\sim 1 \mathrm{~V}$, therefore modulators should attain this value for efficient integration of photonic components with advanced CMOS electronics.

Rise and fall time: The rise and fall time are respectively the time interval for the rising edge of a transmitted optical bit to transition from $10 \%$ to $90 \%$ and $90 \%$ to $10 \%$ of the maximum transmitted power " $P_{\text {on }}$ ".

3dB electro-optical roll-off bandwidth (GHz): In digital optical communications, the data must be transmitted through a system with minimum distortions. To achieve high quality in signal transmission, the $3 \mathrm{~dB}$ EO roll-off bandwidth of individual active components (modulator, photodetectors...) must match the targeted transmission data rate (in Gbits/s). For non-return-to-zero (NRZ) encoded data the optimum bandwidth is comprised between 0.75 and 0.8 times the data rate. In the case of $\mathrm{EO}$ modulators, this $3 \mathrm{~dB}$ drop in power transmission usually results from the limited rise and fall time of the device and/or RF electrode performance. While long rise and fall times distort the transmitted signal, increase inter-symbol-interferences (ISI) and prevent the modulator from responding to the maximum electrical peak to peak amplitude, $\mathrm{RF}$ losses attenuate the effective voltage applied to the device. Note that the $3 \mathrm{~dB}$ roll-off bandwidth is related to the rise and fall time by:

$3 d B B W=0.35 / \max \left(t_{\text {rise }}, t_{\text {fall }}\right)$. 
Data transmission rate (Gbits/s): The data rate that can be transmitted by a given modulator stands for its capacity to encode an electrical signal onto an optically modulated waveform at a given speed. In the case of NRZ binary data encoding, the maximum data rate that a modulator can support is usually limited to 1.25 to 1.33 times its $3 \mathrm{~dB}$ roll-off bandwidth. For instance, a modulator featuring a $30 \mathrm{GHz}$ bandwidth is capable of transmitting NRZ data streams at 40 Gbits/s (i.e. 40,000,000,000 bits/s).

Bit error rate (BER): The performance of a digital communication system is measured by the probability of error per bit, which is referred to as the bit error rate (BER). The BER value is the probability of mistaking " 1 " for "0". A typical acceptable BER is $10^{-9}$ or less (i.e., an average of one error every $1,000,000,000$ bits). The BER measurement represents a critical performance evaluation test to properly assess the maximum achievable data rate of the modulator in real digital transmission systems. Depending on the requirement, a BER lower than $10^{-9}$ may require the use of forward-error-correction (FEC), which may increase the complexity of the overall transmission system.

Eye diagram: In digital communications, an eye diagram is a pattern formed by an oscilloscope, which consists of repetitively sampled, superimposed (usually) random data transitions produced by a given device. In the case of an EO modulator driven under an electrical pseudo-random-bit-sequence ( $\mathrm{PRBS}^{-1}$ ), the modulated optical signal is photodetected (i.e. converted back into an electrical signal) and sampled repetitively in a synchronous manner by means of an external trigger. The eye shape gives useful indications on the performance in a digital transmission system. Qualitatively, an open eye is a good indicator of the modulation capabilities of the characterized optical modulator. Eye closure (or distortion) may be the results of bandwidth limitations, i.e. too long rise and fall time compared to the electrical bit transitions ("0" to " 1 ", " 1 " to " 0 "), noise of the overall system, reflections due to imperfect electrical termination etc... Additionally, an eye diagram provides useful quantitative information on the performance of a modulator such as, rise time and fall time, extinction ratio, signal-to-noise ratio (SNR), jitter etc...

To illustrate the 3dB EO roll-off bandwidth limitations and its impact on the eye shape, four eye diagrams that would theoretically be achieved in modulators with variable $3 \mathrm{~dB}$ EO bandwidths (respectively, 10, 20, 30 and 40 
$\mathrm{GHz}$ ) driven by a NRZ electrical $\mathrm{PRBS}^{-1}$ signal at $40 \mathrm{Gbits} / \mathrm{s}$, are represented. As can be observed, the resultant eye patterns are clearly distorted below $30 \mathrm{GHz}$ 3dB EO roll-off bandwidth, which would affect the device throughput performance in real digital communication systems.
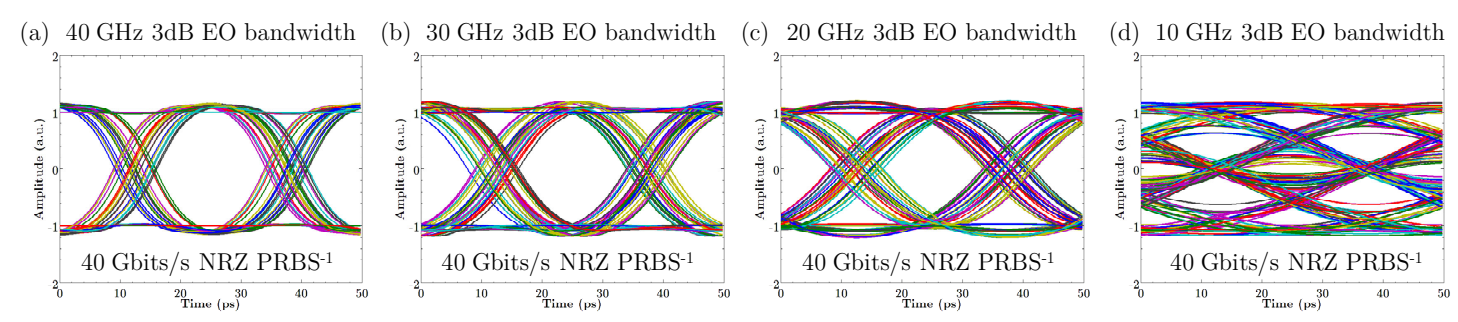

Lower $3 d B$ EO Bandwidth

Figure 2-4: Theoretical eye patterns of optical modulators featuring respectively (a) 40 $\mathrm{GHz}$, (b) $30 \mathrm{GHz}$, (c) $20 \mathrm{GHz}$, and (d) $10 \mathrm{GHz} 3 \mathrm{~dB}$ EO bandwidths and driven under a NRZ PRBS ${ }^{-1} 40$ Gbits/s electrical signal [15].

Optical bandwidth: The optical bandwidth or operating wavelength of an optical modulator is defined as the range of wavelengths range in which the performance of the modulator is maintained. Commercial $\mathrm{LiNbO}_{3}$ modulator feature optical bandwidth of $\sim 80 \mathrm{~nm}$ approximately. Commercial Electroabsorption (EA) modulators have usually operating bandwidth on the order of $\sim 40 \mathrm{~nm}$.

\subsubsection{Amplitude modulator basic parameter calculation}

To introduce the working principle of a typical amplitude modulator, we will describe the basic concepts through a concrete example. As mentioned previously, commercial $\mathrm{LiNbO}_{3}$ amplitude modulators are based on symmetric MZIs with one phase shifter in both arms. The input optical power $P_{\text {in }}$ injected into the device is split ideally equally $\left(0.5 P_{\text {in }}\right.$ or $\left.P_{\text {in }}(\mathrm{dB})-3 \mathrm{~dB}\right)$ by a passive splitting component. If no bias voltage is applied to both phase shifters, the light waves propagating through the arms do not experience any alteration of their phase and recombine at the output of the MZI to form a constructive interference pattern. The modulator is "turned on" and transmits a "1". By contrast, if a bias voltage is applied to one of the phase shifter, light propagating through the altered waveguiding medium experiences a phase shift (here via electro-optic effect) and recombine destructively with the light wave propagating through the other arm and which phase remains unchanged. The modulator is "turned off" and 
transmits a "0". Indeed, the amplitude of the destructive interference depends of the phase shift. Complete extinction is normally achieved when the phase shift difference reaches $\pi$ if no absorption takes place. The required voltage (respectively, length) to accomplish such a phase difference is called $V_{\pi}$ or halfwave voltage (respectively, $L_{\pi}$ or interaction length). The illustration below depicts the basic modulation principle of a MZI.

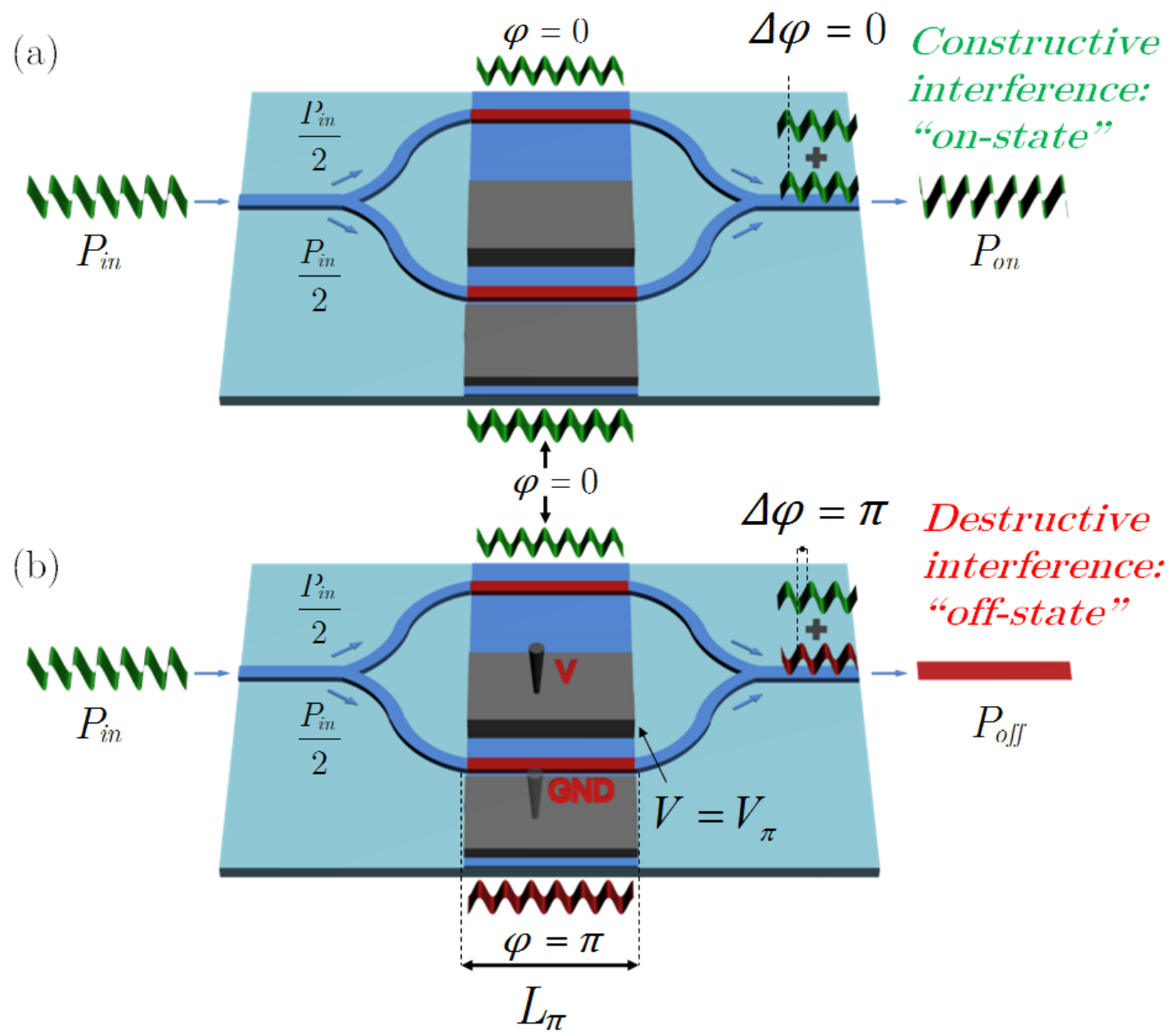

Figure 2-5: Basic principle of operation of an EO MZI modulator (a) The phase shifters (in red) are unbiased and the propagating waves experience no phase difference. Both light waves recombine at the MZI output in the form of a constructive interference. The EO modulator is the "on-state" (b) One of the phase shifter is biased and the wave propagating through the altered waveguiding medium experiences a phase shift ( $\pi$ for maximum extinction). Both light waves recombine at the MZI output in the form of a destructive interference. The EO modulator is the "off-state"

Phase shift: The phase shift experienced by the lightwave propagating within the biased EO medium is given by: 


$$
\Delta \varphi=\frac{2 \pi L}{\lambda} \Delta n_{e f f}
$$

Where $L$ is the phase shifter length, $\lambda$ is the wavelength, and $\Delta n_{\text {eff }}$ is change in effective index of the propagating mode.

Interaction length: Additionally, one may calculate the length required to achieve a $\pi$-phase shift as:

$$
\Delta \varphi=\pi \Rightarrow L_{\pi}=\frac{\lambda}{2 \Delta n_{e f f}}
$$

For instance, assuming that a light wave with $1.55 \mu \mathrm{m}$ wavelength experiencing a effective refractive index change of $1.10^{-3}$, the length required to achieve a $\pi$-phase shift is $L_{\pi} \sim 775 \mu \mathrm{m}$.

Modulation efficiency ( $\boldsymbol{V}_{\boldsymbol{\pi}} \boldsymbol{L}_{\boldsymbol{\pi}}$ product): To compare the performance of the EO phase modulators among the variety of existing devices, a useful figure of merit, which relates the length required to achieve a $\pi$-phase shift $L_{\pi}$ and the halfwave voltage $V_{\pi}$ is universally found in the literature. The modulation efficiency, a reliable and universal comparative figure, is defined as the $V_{\pi} L_{\pi}$ product. If the effective index change versus voltage follows a linear trend, which is the case for $\mathrm{LiNbO}_{3}$ modulators based on the Pockels effect, the $V_{\pi} L_{\pi}$ product is constant. In other words, shorter modulation lengths will be achieved at the expense of higher drive voltage. In silicon modulators, this variation is generally not linear due to the non-linear dependence of the physical effect upon applied voltage. In that case, the modulation efficiency is dependent upon the applied voltage.

Chirp: Chirp in optical modulators arises from the Kramer-Kronig coupling between the real and the imaginary part (absorption coefficient) of the refractive index shown below[16].

$$
\Delta n(\omega)=(c / \pi) P \int_{0}^{\infty} \frac{\Delta \alpha\left(\omega^{\prime}\right) d \omega^{\prime}}{\omega^{\prime^{2}}-\omega^{2}}
$$


Where $\omega$ is the photon frequency, $c$ is the speed of light in a vacuum, and $P$ is the Cauchy principal value. From Equation (1.54), one can observe that any change in the absorption coefficient of a given material will give rise to a change in real refractive index (which produces hence phase shift) and vice-versa. Therefore, amplitude modulators are subject to frequency chirping, i.e. (the instantaneous frequency of a light pulse is time dependent) and phase modulators experience amplitude fluctuations. Therefore, chirped light pulses broaden in dispersive media such as optical fibers. Because pulse broadening increase with distance, for long-haul and high bit rate applications the chirp parameter must be zero (chirp-free) or in some cases non-zero, depending on the travel distance and the fiber dispersion coefficient, which may be used to achieve some amount of pulse compression.

Switching energy and energy per bit: For digital optical modulators, the switching energy required to make a transition from " 0 " to " 1 " is given as:

$$
E_{s}=C V_{p p}^{2}
$$

Where $\mathrm{C}(F)$ is the capacitance of the electrical line and $V_{p p}(V)$ is the voltage applied to the modulator. This is equivalent to when the line experiences a charge $Q(C)$. The line consumes an energy $E=Q . V_{p p}$. In an optical modulator, no energy is required to make the remaining transitions, i.e. "0" to "0", " 1 " to " 1 ", " 1 " to "0", assuming negligible leakage current within the device. In a NRZ PRBS signal, the four types of transitions have equal probability to take place (0.25). Consequently, the energy per bit is $1 / 4$ of the switching energy $\left(E_{s}\right)$. Alternatively, the energy per bit may also be calculated as the average power RF consumed by the modulator, i.e. $1 / 4$ of the total required RF power in the case of a NRZ PRBS signal plus the required DC tuning power (where appropriate) divided by the bit rate:

$$
E(p J / \text { bit })=\frac{\text { Average RF power }(m W)+D C \text { tuning power }(m W)}{\text { Bit rate }(\text { Gbit } / s)}
$$

Example: In a $Z_{0}=50 \Omega$ impedance matched MZI EO modulator (ex: Travelling wave (TW) modulator) operating under a NRZ PRBS signal, the average RF 
power may be estimated as $1 / 4$ of the total power defined as $P_{t o t}=V_{p p}{ }^{2} / Z_{0}$. Assuming that the DC tuning power, which is the power required to adjust the relative phase in both arms of a MZI modulator, or the resonant wavelength of a $\mathrm{RR}$, is zero, the energy per bit may be calculated as:

$$
\text { E.bit }{ }^{-1}=\frac{1}{4} \cdot \frac{V_{p p}^{2}}{Z_{o} . \text { Bit rate }}
$$

Alternatively, the energy per bit consumption of the device may be estimated from the capacitance value of the line as:

$$
\text { E.bit }^{-1}=\frac{1}{4} \cdot C V_{p p}^{2}
$$

An estimate of what should be the required optical interconnect system energy per bit to be competitive with current and near-future off-chip electrical interconnects is $<1 \mathrm{pJ} /$ bit, and $<100 \mathrm{fJ} /$ bit to provide sufficient advantage. For on-chip interconnects, it should be much lower than 50 to 200fJ/bit[2]. This means that for a single external modulator, which is only one building block among others (Lasers, detectors are also part of the interconnect system), the required energy per bit would be 10fJ/bit to a few tens of fJ/bit. For a TW modulator, achieving such low values would only be possible by either decreasing the drive voltage to values lower than $\sim 1 \mathrm{~V}$, which are actually required for CMOS compatible technologies, and/or by increasing the bit rate.

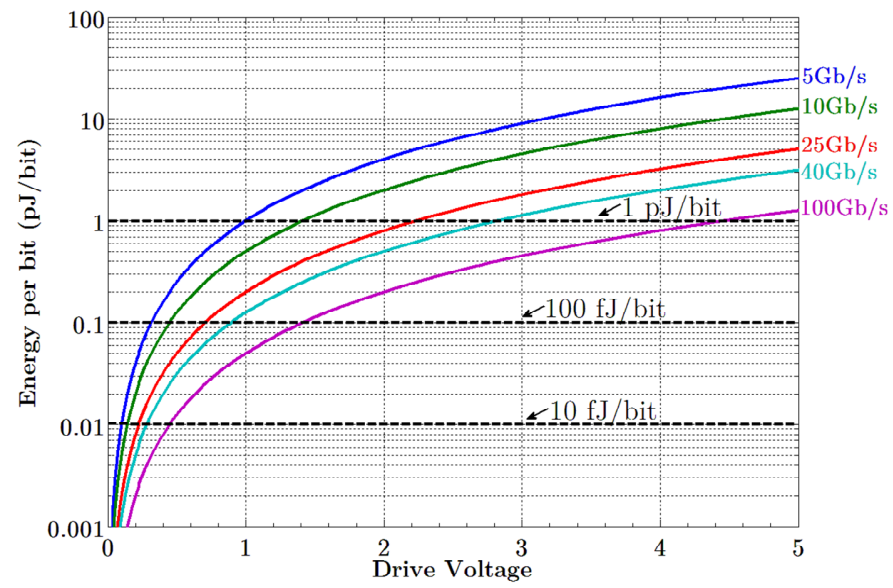

Figure 2-6: Projected Energy per bit consumption of a $50 \Omega$ impedance matched TW MZI modulator versus drive voltage for varying bit rate. 
Figure 2-6 and Table 2-1 illustrate the energy per bit dependence upon drive voltage for a $50 \Omega$ impedance matched modulator. As can be observed, assuming a $1 \mathrm{~V}$ drive voltage, the modulator alone consumes more at and below 40 $\mathrm{Gb} / \mathrm{s}$ rate $(>125 \mathrm{fJ} / \mathrm{bit})$, than the required system energy for off-chip interconnects $(\sim 100 \mathrm{fJ} / \mathrm{bit})$. Decreasing further the drive voltage to $0.5 \mathrm{~V}$ would theoretically enable the modulator to attain the required system energy per bit target from 25 Gbit/s data rate $(<50 \mathrm{fJ} / \mathrm{bit})$ and beyond for both off and chip interconnects. It is worth noting that these figures are only estimations of the potential requirements of optical interconnects and might be subject to variations in the coming years on the basis of CMOS device technology evolution. Therefore, they should not be taken as absolute targets as they might vary depending upon the application field.

\begin{tabular}{|c|c|c|}
\hline Bit rate & Drive voltage & Energy per bit \\
\hline $5 \mathrm{~Gb} / \mathrm{s}$ & $1 \mathrm{~V}$ & $1000 \mathrm{fJ} / \mathrm{bit}$ \\
\hline $10 \mathrm{~Gb} / \mathrm{s}$ & $1 \mathrm{~V}$ & $500 \mathrm{fJ} / \mathrm{bit}$ \\
\hline $25 \mathrm{~Gb} / \mathrm{s}$ & $1 \mathrm{~V}$ & $200 \mathrm{fJ} / \mathrm{bit}$ \\
\hline $40 \mathrm{~Gb} / \mathrm{s}$ & $1 \mathrm{~V}$ & $125 \mathrm{fJ} / \mathrm{bit}$ \\
\hline $100 \mathrm{~Gb} / \mathrm{s}$ & $1 \mathrm{~V}$ & $50 \mathrm{fJ} / \mathrm{bit}$ \\
\hline $5 \mathrm{~Gb} / \mathrm{s}$ & $0,5 \mathrm{~V}$ & $250 \mathrm{fJ} / \mathrm{bit}$ \\
\hline $10 \mathrm{~Gb} / \mathrm{s}$ & $0,5 \mathrm{~V}$ & $125 \mathrm{fJ} / \mathrm{bit}$ \\
\hline $25 \mathrm{~Gb} / \mathrm{s}$ & $0,5 \mathrm{~V}$ & $50 \mathrm{fJ} / \mathrm{bit}$ \\
\hline $40 \mathrm{~Gb} / \mathrm{s}$ & $0,5 \mathrm{~V}$ & $31 \mathrm{fJ} / \mathrm{bit}$ \\
\hline $100 \mathrm{~Gb} / \mathrm{s}$ & $0,5 \mathrm{~V}$ & $12,5 \mathrm{fJ} / \mathrm{bit}$ \\
\hline
\end{tabular}

Table 2-1: Summary of the projected Energy per bit consumption of a $50 \Omega$ impedance matched TW MZI modulator meeting CMOS drive voltage requirements $(<1 \mathrm{~V})$.

\subsubsection{Conclusion}

The basic concepts and parameters presented above will serve as references for the work exposed further on in this thesis. In what follows, we will therefore introduce the physical mechanisms that may be used to achieve efficient optical modulation in silicon. 


\subsection{Silicon as an Efficient Material for Optical Modulation}

Nowadays, the growing trend of carrying information over much shorter distances coupled to the foreseen microelectronics copper interconnect bottleneck has pushed researchers to move towards silicon as an alternative photonic material [17]. Although it is well known that silicon is not naturally efficient for coherent light emission purpose owing to its indirect band gap, and neither has intrinsic linear Pockels effect (typically used in $\mathrm{LiNbO}_{3}$ modulators), pioneering works demonstrated that Raman lasing [18] and high speed modulation [19] is achievable in this material. Naturally, improvements in terms of performance and consumption are underway to meet the stringent specifications of the industrial environment. Therefore, what makes silicon so attractive for light processing applications lies basically in its capacity to meet the ever increasing demand for high-bandwidth technologies, via ultra fast, energy efficient, small footprint optical networks-on-chip and above all, cost-effectiveness compared to its III-V compound counterparts.

Since modulation is a key aspect to achieve these long-standing goals, we introduce in the following the main modulation techniques that may or may not be applied to silicon photonics technology.

\subsection{Physical Mechanisms for Optical Modulation in Silicon}

As mentioned earlier optical modulation can be achieved via two main physical mechanisms:

- Electro-refraction is achieved through changing the real part of the material's refractive index.

- Electro-absorption is achieved through changing the imaginary part of the material's refractive index.

These effects originate from different physical mechanisms which intensity (or existence) depends on the considered material. Since we are particularly interested 
in silicon, we will briefly discuss the suitability of each technique for achieving efficient modulation in this material.

\subsubsection{Electro-optic effects}

The electro-optic effect is basically the mechanism through which the refractive index of a material is changed under an applied electric field. After solid state theory, this is the results of bond charge redistribution throughout the material. Hence, the resulting change in the optical impermeability $\eta$ tensor under an applied electric field $E$ produces the refractive index change induced by the electro-optic effect [20], which respective linear (Pockels) and quadratic (Kerr) coefficients $r_{i j k}$ and $s_{i j k l}$ may be written as:

$$
\Delta\left(\frac{1}{n_{x, y, z}^{2}}\right)=\Delta \eta_{i j}=\eta_{i j}(E)-\eta_{i j}(0)=r_{i j k} E_{k}+s_{i j k l} E_{k} E_{l}
$$

$n_{x, y, z}$ is the refractive index and $E_{k / l}$ are the electric field components in Cartesian coordinates $x, y$, and $z$. While the linear electro-optic coefficient usually dominates over the quadratic one when both are present in a given medium, it vanishes when the crystalline material exhibits inversion symmetry and only the Kerr effect is leftover. $\mathrm{LiNbO}_{3}$, which has a non-centrosymmetric structure features a high Pockels $r_{33}$ coefficient and can be hence used as a refractive index modulating medium. Indeed, most of the high speed commercial electro-optic modulators are based on this material. By contrast, silicon is a centrosymmetric material owing to its diamond crystalline structure and thus exhibits no linear Pockels effect. As a result, only the Kerr effect remains, which relative weakness has been overcome via efficient all-optical experiments in waveguiding structures with strong light confinement and its use has been demonstrated for some non-linear applications [21]. However, because silicon is subject to strong two-photon absorption, which limits the intensity of the Kerr effect, a figure of merit valid for any material has been defined in reference [22]. Alternatively, it is however possible to achieve a Kerr induced refractive index change under an external applied electric field. The absolute refractive index change has been estimated and reaches $\Delta n=1.10^{-6}$ and $\Delta n=1.10^{-4}$ for applied electric field values of respectively $E=10^{5} \mathrm{~V} / \mathrm{cm}$ and $E=10^{6}$ $\mathrm{V} / \mathrm{cm}$ at $1.3 \mu \mathrm{m}$ One should remind however that the breakdown electric field of 
silicon at a concentration of $N_{i}=10^{15} \mathrm{~cm}^{-3}$ is $E=4.10^{5} \mathrm{~V} / \mathrm{cm}$, hence potential silicon devices based on the electric field induced Kerr effect would be limited to low refractive index changes[16]. In 2006, electro-optical effects in silicon have been induced through breaking its crystalline symmetry [23].

\subsubsection{Franz-Keldish effect}

In general, bulk semiconductors can absorb photons, which incident energies are equal to or above that of the material's band gap. However, it was predicted that under a sufficiently strong external electric field, the valence and conduction bands may tilt allowing the electron and hole wave functions to extend within the band gap. As a result, transitions requiring energies lower than the band gap can occur via photon assisted tunneling. In other words, under no applied electric field, the semiconductor is transparent. However, when a bias is applied to the semiconductor the effective band gap decreases causing more photons to be absorbed and hence the material to become opaque as shown in Figure 2-7. This physical phenomenon is known as the Franz-Keldish effect.

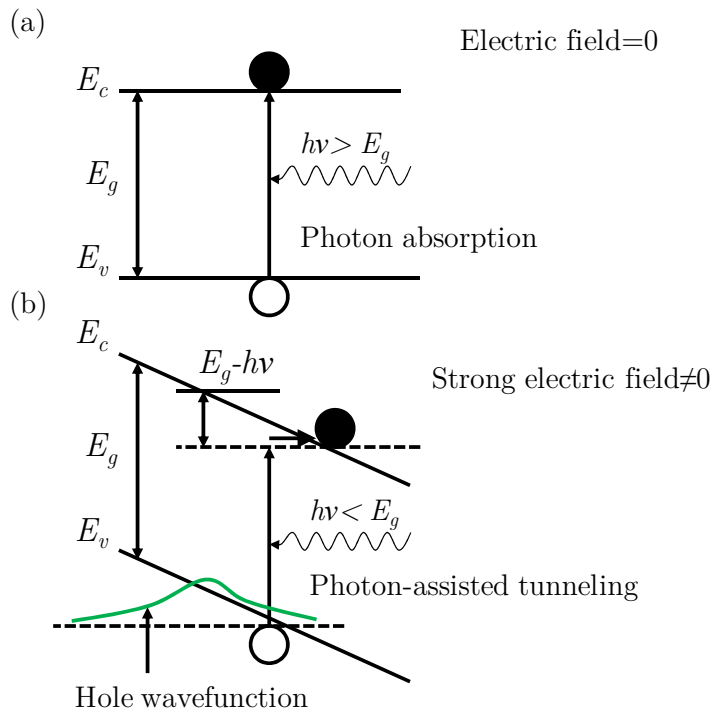

Figure 2-7: (a) Fundamental photon absorption process in a bulk semiconductor at thermal equilibrium. Electronic transitions occur at photon energies equal to or higher than the band gap (b) The energy band tilting under a strong electric field yields to the Franz-Keldysh absorption process. Electronic transitions occur at photon energies lower than the band gap $E_{g}$.

This effect is mostly used for III-V compound-based electro-absorption modulators. Commercial versions of this type of device are normally integrated 
along with a DFB laser. In silicon, however, the Franz-Keldish effect is weak at telecommunication wavelengths. Electro-absorption reaches a maximum at $\lambda=1.07 \mu \mathrm{m}$ under an applied electric field of $E=10^{5} \mathrm{~V} / \mathrm{cm}$. The refractive index change at this point is $\Delta n=1.3 .10^{-5}$. At $\lambda=1.55 \mu \mathrm{m}$, the refractive index change is on the order of $\Delta n=2 \cdot 10^{-6}$ under the same applied electric field. Assuming a quadratic dependence of the refractive index change upon the applied electric field, we may extrapolate and calculate the achieved refractive index change as $\Delta n=7.10^{-5}$ for an applied electric field $E=2.10^{5} \mathrm{~V} / \mathrm{cm}$.

Considering a specific example, we may imagine a $0.220 \mu \mathrm{m}$ high intrinsic (dopant concentration reaching $N_{i}=10^{15} \mathrm{~cm}^{-3}$ ) silicon layer with one electrode on top and another beneath. In such a geometry, the applied voltage to achieve an effective index change of $\Delta n=7.10^{-5}$ is $4.4 \mathrm{~V}$. However, this situation is far from realistic since on one hand, we lie dangerously close to the electric field breakdown of silicon at this concentration (again, $E=4.10^{5} \mathrm{~V} / \mathrm{cm}$ at $N_{i}=10^{15} \mathrm{~cm}^{-3}$ ) and on the other hand, it is not desirable to deposit a metal layer too close to the waveguiding region owing the increased absorption loss. Additionally, in a real case, both top and back contact would be deposited on top of $\mathrm{SiO}_{2}$ layers, which would increase the required voltage to achieve the same refractive index change.

To achieve efficient electro-absorption modulation, hybrid germanium/silicon modulators based on the Franz-Keldish effect were demonstrated [24]. A pure Germanium modulator was also reported based on this effect [25].

\subsubsection{Quantum confined stark effect}

Quantum confined stark effect (QCSE) is based on strong excitonic phenomena that changes the absorption edges of materials. To achieve efficient electroabsorption multi-quantum well (MQW) structures consisting of a stack of thin layers of two materials with different band gap energy (typically InGaAs/InAlAs) are realized. As an external electric field is applied perpendicular the quantum wells, the bands tilt and the electron and hole wavefunctions spatially separate. As a result, the electron subband energy $E_{e}$ increases while the hole subband energy $E_{h}$ decreases and the difference between these two energies $\left(E_{c}-E_{h}\right)$ reduces accordingly. In addition, the exciton binding energy $\mathrm{E}_{e x}$, which is high in quantum well structures, decreases also. As a result, because the effective bandgap decreases, the energy required to make an electronic transition is lower under an 
applied electric field (that is when, $E_{\operatorname{tran}(E>0)}<E_{\operatorname{tran}(E=0)}$ ) and the absorption edge of the quantum well structure is shifted towards longer wavelengths. QCSE-based modulators are very efficient and compact devices and can be monolithically integrated with a DFB laser. Commercial devices working at 10 Gbit/s are available. Much higher modulation speeds are however achievable [26].

QCSE was also demonstrated in Si/SiGe MQW structures [27]. The device showed strong absorption coefficient and band edge shift, which makes it comparable with current III-V compound QCSE-based modulators. One should mention however that QCSE are usually thick $(\sim 1 \mu \mathrm{m})$ and this makes light coupling from a silicon nanowire to a QCSE modulator challenging.

(a)

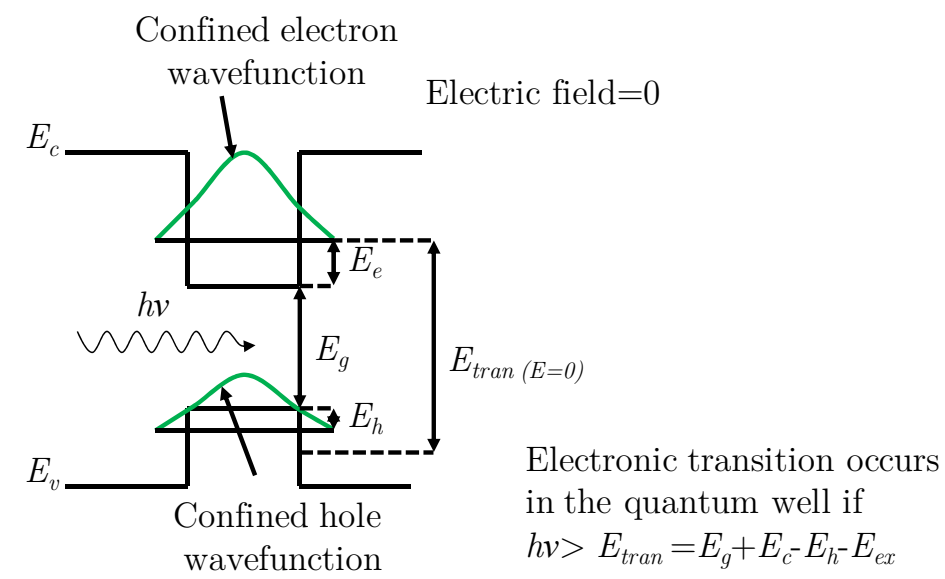

(b)

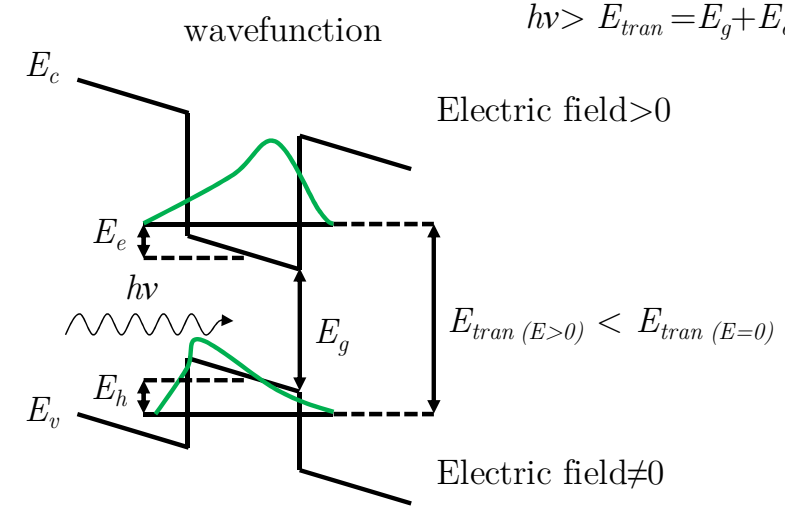

Figure 2-8: Schematic description of the Quantum Stark effect (a) Quantum well under no applied electric field (b) Quantum well under an applied electric field; the energy required to make a transition $E_{\text {tran }}$ decrease and the absorption edge is shifted towards longer wavelengths.

\subsubsection{Thermo-optic effect}

The high thermo optic coefficient (Equation (1.60)) of silicon enables large refractive index changes. However, this is a rather slow effect as it occurs on time 
scale on the order of hundreds of nanoseconds to microseconds. As a result, thermo-optic effect is clearly not suitable for high speed purposes but rather for applications where speed is not critical. The change in refractive index in silicon produced by temperature variations is given by:

$$
\frac{\Delta n}{\Delta T}=1.86 .10^{-4} /{ }^{o} C
$$

As an example, a temperature variation of only $10{ }^{\circ} \mathrm{C}$ may lead to local refractive index variation of $\Delta n=1.86 .10^{-3}$, which is more than one order of magnitude higher than the maximum theoretical refractive index variation achieved via either the Franz-Keldish effect or Kerr effect in pure silicon. While silicon's high thermo-optic coefficient is beneficial in terms of thermo-optical control, it may become a drawback due to the high sensitivity of photonic devices to low temperature changes. As a result, thermal management is a key aspect of silicon photonics-based devices

\subsubsection{Plasma dispersion effect}

The plasma dispersion effect is the result of free carriers changing the refractive index and absorption coefficient of a given semiconductor. According to the predictions of Drude's model and summing the contribution of free electrons and holes the total change in refractive index and optical absorption is given by [16]:

$$
\begin{aligned}
& \Delta n=-\frac{e^{2} \lambda^{2}}{8 \pi^{2} c^{2} \varepsilon_{0} n}\left(\frac{\Delta N_{e}}{m_{e}}+\frac{\Delta N_{h}}{m_{h}}\right) \\
& \Delta \alpha=\frac{e^{3} \lambda^{2}}{4 \pi^{2} c^{3} \varepsilon_{0} n}\left(\frac{\Delta N_{e}}{m_{e}}+\frac{\Delta N_{h}}{m_{h}}\right)
\end{aligned}
$$

Where $e$ is the electronic charge, $\lambda$ is the wavelength, $c$ is the speed of light in a vacuum, $\varepsilon_{0}$ is the permittivity of free space, $n$ is the refractive index of unperturbed silicon, $m_{e}$ and $m_{h}$ are respectively the effective masses of electrons and holes, $\mu_{e}$ and $\mu_{h}$ are the respective mobilities of electrons and holes, $\Delta N_{e}$ and $\Delta N_{h}$ are respectively the variations in concentration of electrons and holes. Taking $m_{e}=0.26 m_{0}$ and $m_{h}=0.39 m_{0}$ which are the actual conductivity effective masses of 
electrons and holes, and mobility values at $300 \mathrm{~K} \mu_{e}=1400 \mathrm{~cm}^{2} . \mathrm{V} \cdot \mathrm{s}^{-1}$ and $\mu_{h}=450$ $\mathrm{cm}^{2}$.V. $\mathrm{s}^{-1}$, we can derive the predicted changes in refractive index and absorption induced by electron and holes as a function of their respective concentration variations. As a comparison, we may contrast Drude's theoretical calculations with Soref's empirical experimental results [16] that describe the change in refractive index and optical absorption via the following relationships:

$$
\begin{aligned}
& \Delta n=-8.8 \times 10^{-22} \Delta N_{e}-8.5 \times 10^{-18} \Delta N_{h}^{0.8} \\
& \Delta \alpha=8.5 \times 10^{-18} \Delta N_{e}+6.0 \times 10^{-18} \Delta N_{h}
\end{aligned}
$$

where $\Delta N_{e}$ and $\Delta N_{h}$ are respectively the variations in concentration of electrons and holes in $\mathrm{cm}^{-3}$. The first observation that can be made is that the refractive index change induced by the electrons is linearly dependent upon their concentration (or density). This linear dependence is also found in the Drude model case. However, the refractive index change induced by holes is sub-linearly dependent upon their concentration within the semiconductor in contrast with the Drude case. In both case, the change in absorption follows a linear tendency, but electrons absorb more light in the case of Soref's model. Figure 2-9 below depicts these variations and allows us to observe a certain amount of discrepancy between the two models.
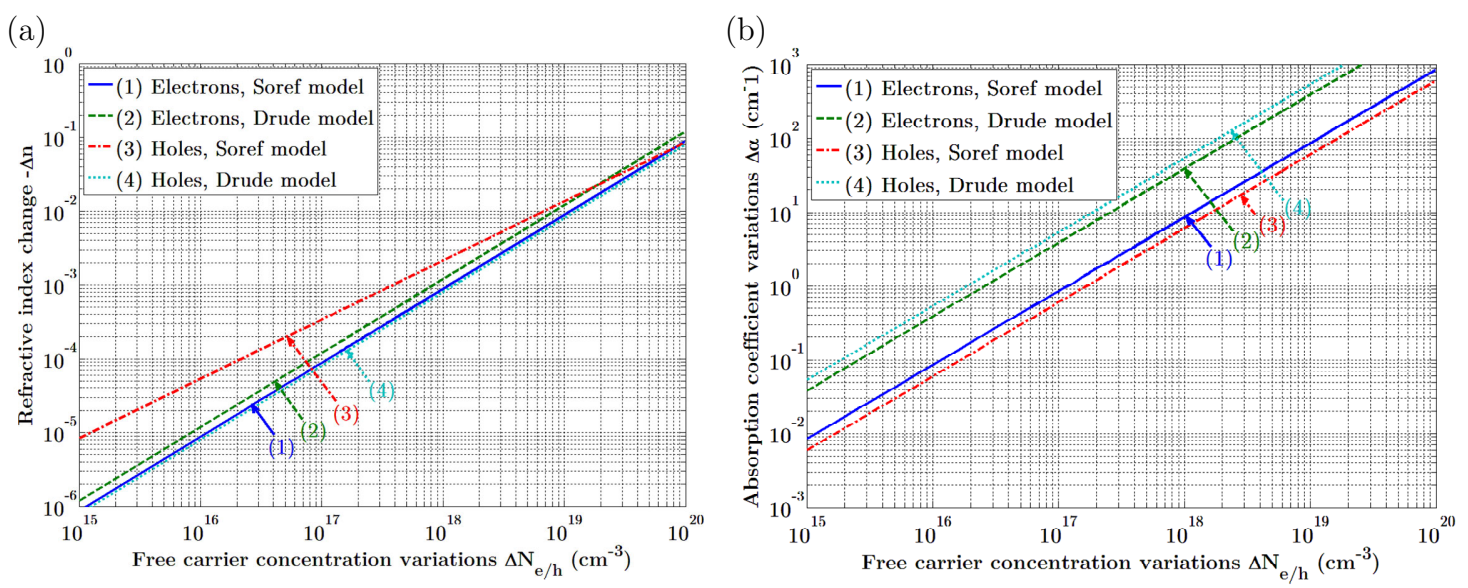

Figure 2-9: (a) Refractive index change versus free carrier concentration variations calculated from the Soref's empirical model (respectively Drude's theoretical model) induced by electrons (1) (resp. (2)) and holes (3) (resp. (4). (b) Absorption coefficient versus free carrier concentration variations calculated from the Soref's empirical model (respectively Drude's theoretical model) induced by electrons (1) (resp. (2)) and holes (3) (resp. (4)). 
As can be seen, while the Drude model predicts that electrons are slightly more efficient in changing the refractive index than holes do, Soref's experimental results expose a completely different picture. Indeed, holes are clearly more efficient than electrons in changing the refractive index for carrier concentrations up to $10^{19} \mathrm{~cm}^{-3}$. Electrons become then slightly more electro-refractive than holes. However, in practice, reaching such high concentration variations is on one hand extremely difficult to achieve and may on the other hand lead to high optical loss. Regarding the absorption, the two models are in clear disagreement as Drude's model predicts changes in absorption one order of magnitude higher than the Soref model. Furthermore, while Soref's model shows that hole are less electroabsorptive than electrons, Drude's model foresees the contrary. Overall, refractive index variations on the order of $\Delta n=1.10^{-4}$ up to $\Delta n=2.10^{-3}$ are achievable with corresponding carrier concentration variations around $\Delta N_{h}=2.10^{16} \mathrm{~cm}^{-3}$ to $\Delta N_{h}=1.10^{18} \mathrm{~cm}^{-3}$ at $1.55 \mu \mathrm{m}$ wavelength. Figure 2-10 (a) shows the amount of free holes required to achieve a $\pi$-phase shift over a given length called here $L_{\pi}$ together with the corresponding absorption losses. As can be seen, they increase dramatically from free hole concentration levels of $\Delta N_{h} \sim 2.10^{17} \mathrm{~cm}^{-3}$. However, since high concentrations induce high refractive index changes, one may estimate the length required to achieve a $\pi$-phase shift in a given silicon waveguiding structure, which for obvious reasons will scale accordingly. The corresponding net loss or insertion loss in $\mathrm{dB}$ is calculated as follows:

$$
\text { Net loss }(d B)=-10 \log _{10}\left[\exp \left(-\left(\alpha_{0}+\alpha_{\text {carriers }}\right) L_{\pi}\right)\right]
$$

Where $\alpha_{0}$, is the absorption induced by the waveguiding structure (due to material absorption, sidewall roughness etc..) and $\alpha_{\text {carriers }}$ is the absorption induced by the free carriers. $L_{\pi}$ is the length required to achieve a $\pi$-phase shift. In the previously indicated range of free carrier concentration variations, $L_{\pi}$ values may vary from $7.75 \mathrm{~mm}$ down to $0.4 \mathrm{~mm}$, which interestingly means that the net loss figure induced by free holes remain below $2.5 \mathrm{~dB}$ after Figure 2-10 (b). High concentrations may be achieved via injection of free carriers (say up to $\Delta N_{e / h}=1.10^{18} \mathrm{~cm}^{-3}$ ) while depletion is more restricted to lower amounts. Additionally, in practice, refractive index change in the injection (resp. depletion) regime will preferably be achieved in a pin diode (resp. in a pn junction). This also implies that both carrier types will contribute to the refractive index change. 
Convenient designs are therefore required to make the contribution of one type of carrier to the refractive index change dominant over the other. Let us remind that the values calculated here are made considering that the refractive index change is uniform all over the waveguiding structure. Although this is far from realistic in practice, and hence local refractive index changes must be calculated, injection is generally more efficient than depletion as the free carriers fill a greater volume of material. Depletion is in turn limited to the space charge region width, which implies only partial overlap between free carriers and optical mode. On the other hand, we will see further on that these effects occur on very different time scale. Intrinsically, injection, which relies on minority carrier generation/recombination processes, is relatively slow while depletion, which involves majority carriers, follows a capacitive trend but with very short intrinsic rise and fall times if the $\mathrm{RC}$ constant of the device is kept small.

(a)

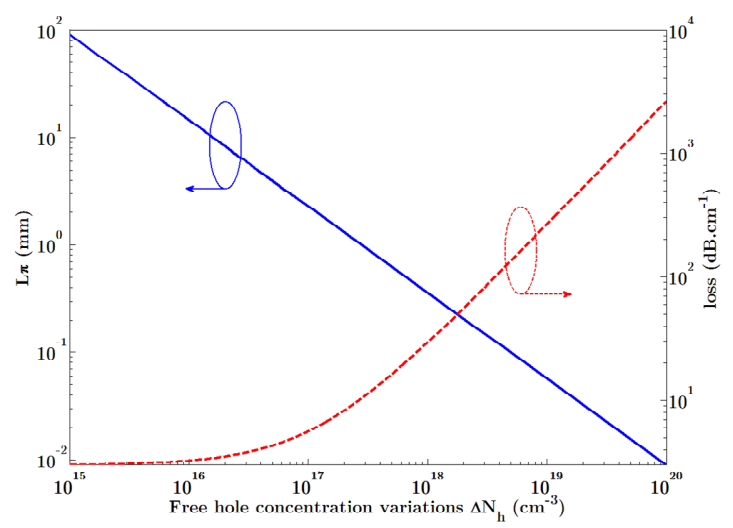

(b)

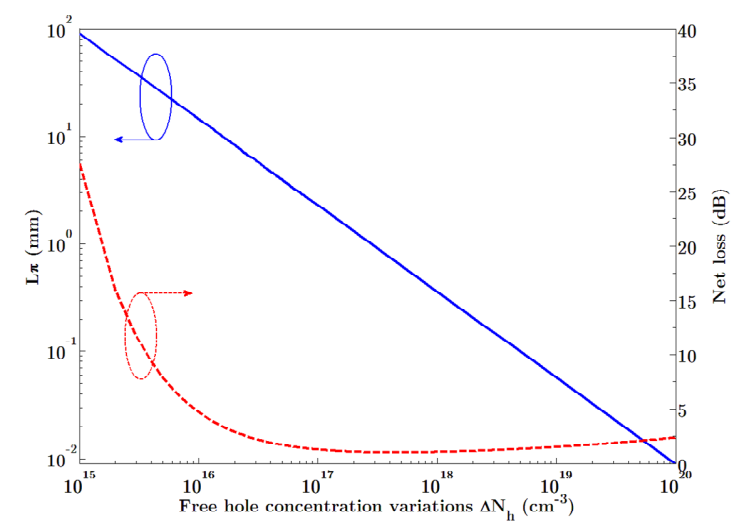

Figure 2-10: (a) $L_{\pi}$ (Length to achieve a $\pi$-phase shift) variations (blue plain line) plotted together with the loss in $\mathrm{dB} \cdot \mathrm{cm}^{-1}$ (red dashed line) induced by free hole concentration variations based on the Soref empirical model. (b) $L_{\pi}$ variations (blue plain line) plotted together with the net loss (red dashed line) induced by free hole concentration variations based on the the Soref empirical model. In both cases, $\alpha_{0}=0.691 \mathrm{~cm}^{-1}$, which is equivalent to $3 \mathrm{~dB} \cdot \mathrm{cm}^{-1}$. It is worth noting that this loss figure is highly dependent upon the fabrication process and/or waveguide dimensions and hence may be lowered. (via decreasing sidewall roughness, for instance).

\subsubsection{Conclusion}

In conclusion, the plasma dispersion effect is to our knowledge one of the most effective mechanism to achieve high speed electro-refraction in silicon with relatively low loss. Well-known electrical structures permit interesting variations 
in free carriers concentrations in almost any given light waveguiding structure, which opens a wide range of possibilities in the field of silicon active devices.

\subsection{Silicon-Based Modulators: The State-of-the-Art}

\subsubsection{Silicon modulators}

Several refractive index change mechanisms in silicon have been presented in the previous section and the majority of them are relatively weak, except to some extent, in the case of the plasma dispersion effect and QCSE. Fortunately, modulation performance is indeed not exclusively dependant on the absolute refractive index change, but rather on the amount of interaction between light and the altered medium. In other words, the performance of real devices will strongly depend upon their capacity to optimize and in some cases enhance lightmatter interactions. Therefore, over the last decade, researchers have demonstrated a variety of modulator architectures in an effort to scale their size and operating drive voltage (hence power) while making them CMOS or nearlyCMOS compatible for cost effectiveness and reliability purposes.

\section{Pockels modulators}

First of all, it has already been mentioned that silicon's natural centro-symmetric crystalline structure rules out any linear electro-optic Pockels effect, conventionally used as a modulation mechanism in commercial $\mathrm{LiNbO}_{3}$ modulators, for instance. In 2006 however, breaking the crystal symmetry by depositing a straining layer on top of a silicon waveguide was demonstrated as a means to induce the linear electro-optic effect [23]. Further evidence of the Pockels effect in strained silicon was reported very recently[28]. Although being the highest reported so far in pure silicon, the electro-optic coefficient is however relatively weak and large drive voltages are hence required to achieve sufficient modulation. Increasing the bandwidth and uniformity of the induced Pockels effect as well as the repeatability of the process is required for industrial applications.

\section{Franz Keldish and quantum confined Stark effect modulators}

Very recently, the combination of CMOS compatible alternative materials such as germanium [24] and graphene [29] with silicon have also been proposed to enable 
electro-optical modulation based on electro-absorption in hybrid devices, however limited so far to a bandwidth of around $1 \mathrm{GHz}$. Recent improvements in the field of Ge electro-absorption modulators, enabled $30 \mathrm{GHz}$ bandwidth and $12.5 \mathrm{Gbit} / \mathrm{s}$ data transmission with adiabatic coupling to thick $(3.5 \mu \mathrm{m})$ silicon waveguides[25]. In addition, strong QCSE in Ge/SiGe MQW structures was also demonstrated as an efficient way to produce electro-absorption modulators [27].

\section{Free carrier plasma dispersion modulators}

Up to date, the free-carrier dispersion effect is the most effective mechanism to achieve high-speed electro-optical modulation in silicon [30]. As seen previously, it is related to the variation in concentration of free carriers in a semiconductor [16]. Since the first silicon modulator exceeding a $1 \mathrm{GHz}$ bandwidth was demonstrated based on this effect [31], numerous devices have been reported by either accumulation [32], injection [33, 34] or depletion [19, 35-37] of free carriers.

Briefly, accumulation modulators are based on biased MOS structure, which accumulate free carriers at the $\mathrm{Si} / \mathrm{SiO}_{2}$ interface. These modulators suffer generally from high capacitance and a lack of modulation efficiency due to the low amount of carrier involved. $10 \mathrm{Gbit} / \mathrm{s}$ operation with $3.8 \mathrm{~dB}$ ER was however demonstrated in a relatively long structure $(\sim 3.5 \mathrm{~mm})$, which modulation efficiency was $3.3 \mathrm{~V} . \mathrm{cm}$.

Injection modulators are usually based on forward biased pin diode, in which a minority carrier current flows through the intrinsic silicon region where the mode propagates. Because of the slow carrier/generation recombination process modulation are generally limited to $1 \mathrm{GHz}$. To counteract such a fundamental limitation, a pre-emphasis driving scheme consisting in applying short peaks of current at the beginning of each electrical bits to accelerate the carrier generation process and followed by a reverse bias operation to extract carriers more efficiently, may been employed. In such a configuration, modulation speeds up to $18 \mathrm{Gbit} / \mathrm{s}$ with $3 \mathrm{~dB}$ ER have been achieved in a ring resonator[38]. Additionally, carrier injection in a $200 \mu \mathrm{m}$ rib waveguide modulator integrated into a MZI operating at $10 \mathrm{Gbits} / \mathrm{s}$ was reported. The short modulation length is due to the device modulation efficiency, $V_{\pi} L_{\pi} \sim 0.036 \mathrm{~V} . \mathrm{cm}$ which is 2 orders of magnitude lower than in the previously mentioned MOS modulator. Injection modulators are simultaneously electro-absorptive (leading to chirp and extra insertion losses) and electro-refractive due to the uniformity of the plasma and the high amount of injected carriers. Special care must be taken to minimize the 
insertion losses and power consumption. Moreover, electrical pre-emphasis adds complexity to the overall device.

Depletion modulators are based on the change in space charge region depletion width of a $p n$ junction and hence involve only majority carriers. Therefore, no generation/recombination process takes place since the modulator behaves as a RC circuit with specific rise and fall time constant. This effect is intrinsically fast but involves a lower amount of carriers than in the case of the injection regime, which implies that carrier depletion-based modulator are generally less efficient than their injection counterparts. Special care in decreasing the access (or series) resistance and decreasing the junction capacitance must be taken especially in the case of lumped electrode configuration. In the case of a TW electrode design, the resistance and capacitance is distributed along the modulator as the electrical and optical signals travel at similar speeds.

40 Gbit/s silicon modulation [19] was achieved in 2008 in a 1mm long travelling device with however low ER $(\sim 1 \mathrm{~dB})$ owing to the lack of efficiency $(\sim 4 \mathrm{~V} . \mathrm{cm})$ of the device and RF losses. More recently, a transmission rate of 40 Gbit/s was reported using both compact (1mm long) and large footprint (3.5mm long) MZI TW modulators based on carrier depletion operation [39]. For the compact version of the device ( $1 \mathrm{~mm}$ long), in spite of a fair modulation efficiency of $\sim 2.7 \mathrm{~V}$.cm, the state-of-the-art bit rate performance was achieved with a lower extinction ratio (ER) of $3.5 \mathrm{~dB}$ making error free modulation, i.e. bit-error-rate $(\mathrm{BER})<10^{-9}$, essentially unviable. It should be pointed out that the BER measurement stands for the critical performance evaluation test to properly assess the maximum data rate of the modulator in real digital transmission systems. A less efficient but high speed (40 Gbit/s) polarization independent (TE $V_{\pi} L_{\pi} \sim 11$ V.cm and TM $V_{\pi} L_{\pi} \sim 14$ V.cm) silicon modulator was also reported at the same time [40].

\section{Free carrier plasma slow wave modulators}

In an effort to increase the interaction between free carriers and optical mode, which is precisely the main line of this work, low power and high level of integration have been achieved in silicon by using photonic crystal ( $\mathrm{PhC}$ )-based MZI modulators [41, 42]. The dispersion of $\mathrm{PhC}$ waveguides offers the possibility to enhance the modal effective index variation by up to 2 orders of magnitude. Therefore, the active length of conventional rib modulators can be reduced by the 
same factor to achieve the phase shift needed for the modulation. This figure of merit, which will be described further on, is called the enhancement factor.

\section{Injection PhC modulators}

The first $\mathrm{PhC}$ modulator was demonstrated in 2005 and showed low speed operation $(300 \mathrm{KHz})$ under a drive voltage of $7.5 \mathrm{mV}$ and for an active length of $80 \mu \mathrm{m}$. The modulation was achieved via free holes injected through a pip diode integrated into a MZI. Two years later, the same group followed up on these results [43] and reported high-speed operation using a PhC-based MZI having similar dimensions. The active element is made up of a pin diode in one arm of a MZI with both p- and n-type concentrations reaching $5 \times 10^{17} \mathrm{~cm}^{-3}$. The authors reported a modulation bit rate of $1 \mathrm{~Gb} / \mathrm{s}$ under a square pattern drive voltage (not PRBS) with ER of $20 \%(\sim 1 \mathrm{~dB})$. Maximum DC ER of $93 \%(\sim 11.5 \mathrm{~dB})$ was obtained with an injection current of $7.1 \mathrm{~mA}$. Interestingly, this level of injection current is similar to a $500 \mu \mathrm{m}$ long rib waveguide modulator reported 10 years earlier[44]. Another PhC-based modulator embedded in a capacitor was also demonstrated [45]. The device features a figure of merit of $0.18 \mathrm{~V} . \mathrm{cm}$, which means that for a $6 \mathrm{~V}$ applied voltage, a pi-phase shift is achieved over a length of $300 \mu \mathrm{m}$. This reduced interaction length is approximately one order of magnitude lower than for conventional rib waveguide MOS and depletion modulators. Maximum DC modulation depth of $90 \%$ was obtained with a $6 \mathrm{~V}$ applied voltage for a group index $n_{g} \sim 100$ at $\lambda=1551 \mathrm{~nm}$. Furthermore, the modulator was driven under a square electrical signal (not PRBS) at 1.6 Gbits/s with $3 \mathrm{~dB}$ extinction ratio and with $n_{g} \sim 50$ at $\lambda=1548 \mathrm{~nm}$. In addition, the low capacitance also enabled the modulator power consumption to be decreased down to $0.54 \mathrm{pJ} / \mathrm{bit}$ at the expense of a lower operating bandwidth and higher losses due to strip WG to $\mathrm{PhC}$ impedance mismatch (5dB/port). Very recently, pre-emphasized 10 Gbits/s operation with $7.2 \mathrm{~dB}$ ER in a $200 \mu \mathrm{m}$-long $\mathrm{PhC}$ modulator embedded in $p n$ junction has been reported [42] in spite of a $3 \mathrm{~dB}$ roll-off frequency of $3 \mathrm{GHz}$. The device feature modulation efficiencies of $V_{\pi} L_{\pi} \sim 0.056 \mathrm{~V} . \mathrm{cm}$ and $0.016 \mathrm{~V} . \mathrm{cm}$ when respectively operated in both depletion and injection regimes and only in the injection regime at a group index of $\sim 18$. BER measurements have been performed and reach $\sim 1.310^{-4}$, which according to the author is beyond the typical threshold $\left(\mathrm{BER}<10^{-3}\right)$ of detection systems that employ forward error correction [46]. While this may be true for some applications, it adds complexity 
(and probably cost) to the overall transmission system. In general $\mathrm{BER}<10^{-9}$, that is error free transmission, is required.

\section{Injection cascaded ring modulators}

In an effort to increase the optical bandwidth of slow wave modulators, a slowlight Mach-Zehnder modulator loaded with ten 7.2 um-radius cascaded ring resonators was reported[47]. The fabricated device operates at $10 \mathrm{~Gb} / \mathrm{s}$ in free carrier injection regime with pre-emphasis with a driving voltage of $1 V_{p p}$ over a wavelength range of $1 \mathrm{~nm}$ (1551-1552nm) and without any adjustment of the resonant wavelengths (no thermal DC tuning). The extinction ratio lies between 3 and $5 \mathrm{~dB}$ over the same bandwidth. The MZI arm length is $478 \mu \mathrm{m}$. Compared to a conventional MZI (i.e. not ring-loaded) the injection current $(4.21 \mathrm{~A})$ is decreased by a factor lying between 4 and 9: 0.86, 0.45 and 1.05 at 1551.0, 1551.5, and $1552.0 \mathrm{~nm}$, respectively.

\section{Depletion laterally corrugated waveguide modulator}

Meanwhile, we demonstrated the first carrier depletion-based slow wave modulator as a means to enhance the modulation efficiency[48]. This result is the central achievement of this thesis. The device is based on a $500 \mu \mathrm{m}$ long periodic laterally corrugated waveguide (LCWG) embedded in a self aligned pn junction [49]. It combines the attractive properties of slow light propagation in a nanostructured periodic waveguide together with a high speed semiconductor $p n$ diode, and exhibits error free modulation up to $20 \mathrm{Gbit} / \mathrm{s}$ with $6.3 \mathrm{~dB}$ ER at group index of $n_{g} \sim 11$. The longer phase shifter features $10 \mathrm{Gbit} / \mathrm{s}$ error free operation with $9.5 \mathrm{~dB}$ ER at the same group index. These results are supported by modulation rate capabilities reaching $40 \mathrm{Gbit} / \mathrm{s}$ (ER 3dB). Modulation efficiencies are $V_{\pi} L_{\pi} \sim 1.27 \mathrm{~V} . \mathrm{cm}$ is obtained for a group index of 11 and as low as $\sim 0.45 \mathrm{~V}$.cm when the group index increases up to $\sim 22$, which is respectively 2 and 5 times greater than in a 400nm reference rib waveguide modulation. All the details are provided in chapter 3 .

\section{Hybrid silicon modulators}

Silicon-III-V compounds hybrid [50] and silicon-organic hybrid (SOH) [51] modulators were shown to exhibit error free modulation beyond 40 Gbit/s. The combination of plasmonics with silicon has also been shown as a promising 
candidate to push even further the limits of electro-optical modulation [52] over ultra short lengths.

\section{Amorphous silicon modulators}

Electro-optical modulation in a reverse biased a-Si:H pin waveguiding structure was also demonstrated recently. The device is operated at large voltages (up to $90 \mathrm{~V}$ ) and features a maximum $V_{\pi} L_{\pi}$ product of $\sim 63 \mathrm{~V} \mathrm{~cm}$. The low temperature involved in the process makes this modulator readily compatible with CMOS processes[53].

\section{Summary}

The following table summarizes the main achievements in the field of silicon modulators classified by both the year of publication and the electrical modulating structure. The "best" and "worst" parameter values for each modulator family are respectively represented in green and red. 


\begin{tabular}{|c|c|c|c|c|c|c|c|c|c|c|c|c|}
\hline Reference & Group & Year & Electrical structure & Optical structure & Modulation efficiency & EO BW & Bit rate & Vpp & Dyn. ER & Loss & Length & Switching energy \\
\hline & & 10 & & & & $\$ 011$ & & & 101010 & & & \\
\hline LIA05-OE [32] & Intel corporation & 2005 & MOS Accumulation & MZI & $3,3 \mathrm{~V} . \mathrm{cm}$ & $10 \mathrm{GHz}$ & $10 \mathrm{G}$ & $3,5 \mathrm{~V}$ & $3,8 \mathrm{~dB}$ & $10 \mathrm{~dB}$ & $3450 \mu \mathrm{m}$ & $66 \mathrm{pJ} / \mathrm{bit}$ (ED) \\
\hline DAN09-OFC [54] & LightWire & 2009 & MOS Accumulation & $M Z I$ & $0,2 \mathrm{~V} . \mathrm{cm}$ & $10 \mathrm{GHz}$ & $10 \mathrm{G}$ & $1,2 \mathrm{~V}$ & $9 \mathrm{~dB}$ & $\mathrm{~N} / \mathrm{A}$ & $800 \mu \mathrm{m}$ & $<3 \mathrm{pJ} /$ bit \\
\hline FUJ10-OFC [55] & NEC & 2010 & MOS accumulation & $\mathrm{MZI}$ & $0,5-0,67$ V.cm & $\mathrm{N} / \mathrm{A}$ & $12,5 \mathrm{G}$ & $3,5 \mathrm{~V}$ & $3-6 \mathrm{~dB}$ & $1,4 \mathrm{~dB}$ & $120 \mu \mathrm{m}$ & $\mathrm{N} / \mathrm{A}$ \\
\hline 00 & 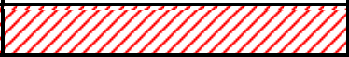 & 1 & 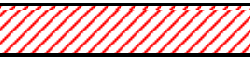 & & & 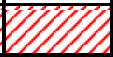 & & & & & & \\
\hline GUN06-IEEE [56] & Luxtera & 2006 & PN Depletion & $\mathrm{MZI}$ & $\mathrm{N} / \mathrm{A}$ & $\mathrm{N} / \mathrm{A}$ & $10 \mathrm{G}$ & $2,5 \mathrm{~V}$ & $5 \mathrm{~dB}$ & $N / A$ & $\mathrm{~N} / \mathrm{A}$ & $N / A$ \\
\hline LIAO07-OE [19] & Intel corporation & 2007 & PN depletion & $\mathrm{MZI}$ & $4 \mathrm{~V} . \mathrm{cm}$ & $30 \mathrm{GHz}$ & $40 \mathrm{G}$ & $6 \mathrm{~V}$ & $1 \mathrm{~dB}$ & 1,8 & $1000 \mu \mathrm{m}$ & $16 \mathrm{pJ} / \mathrm{bit}(\mathrm{ED})$ \\
\hline WAT08-GIV [57] & Sandia national labs & 2008 & PN depletion & Microdisks & $\mathrm{N} / \mathrm{A}$ & $\mathrm{N} / \mathrm{A}$ & $10 \mathrm{G}$ & $3,5 \mathrm{~V}$ & $\mathrm{~N} / \mathrm{A}$ & $N / A$ & $2 \mu \mathrm{m}(r)$ & $0,085 \mathrm{pJ} / \mathrm{bit}(\mathrm{AD})$ \\
\hline GAR09-OE [35] & UNIS-UPV-IEF-U.St An-U.Ghent* & 2009 & PN depletion & Ring & 7,75 V.cm (2V) & $19 \mathrm{GHz}$ & $\mathrm{N} / \mathrm{A}$ & $\mathrm{N} / \mathrm{A}$ & $N / A$ & $\mathrm{~N} / \mathrm{A}$ & $40 \mu \mathrm{m}(r)$ & $\mathrm{N} / \mathrm{A}$ \\
\hline PAR09-OE [58] & ETRI Korea & 2009 & PN depletion & MZI & $1,8 \mathrm{~V} . \mathrm{cm}$ & $\mathrm{N} / \mathrm{A}$ & $12,5 \mathrm{G}$ & $4 \mathrm{~V}$ & $3 \mathrm{~dB}$ & $3,3 \mathrm{~dB}$ & $750 \mu \mathrm{m}$ & $\mathrm{N} / \mathrm{A}$ \\
\hline GIL09-PTL [59] & Alcatel-Lucent-Bell & 2009 & PN depletion & $M Z I+$ rings & $3 \mathrm{~V} . \mathrm{cm}$ & $35 \mathrm{GHz}$ & $\mathrm{N} / \mathrm{A}$ & $\mathrm{N} / \mathrm{A}$ & $\mathrm{N} / \mathrm{A}$ & $0,5 \mathrm{~dB}$ & $270 \mu \mathrm{m}(\mathrm{r})$ & $\mathrm{N} / \mathrm{A}$ \\
\hline GIL10-JQSTE [60] & Alcatel-Lucent-Bell & 2010 & PN depletion & $M Z I+$ rings & 2V. Cm & $>15 \mathrm{GHz}$ & $10 \mathrm{G}$ & $4 \mathrm{~V}$ & $1,35 \mathrm{~dB}$ & $2 \mathrm{~dB}$ & $280 \mu \mathrm{m}(\mathrm{r})$ & $8 \mathrm{pJ} / \mathrm{bit}$ \\
\hline TSU10-JSTQE [61] & IM. Singapore & 2010 & PN depletion & MZI & 2,56 V.cm & $N / A$ & $10 \mathrm{G}$ & $5 \mathrm{~V}$ & $6,1 \mathrm{~dB}$ & $2 \mathrm{~dB}$ & $2000 \mu \mathrm{m}$ & $12,5 \mathrm{pJ} / \mathrm{bit}(\mathrm{ED})$ \\
\hline FEN10-OE [62] & Kotura & 2010 & PN depletion & MZI & 1,4 V.cm & $12 \mathrm{GHz}$ & $12.5 \mathrm{G}$ & $8 \mathrm{~V}$ & $7,2 \mathrm{~dB}$ & $1,9 \mathrm{~dB}$ & $1000 \mu \mathrm{m}$ & $25,6 \mathrm{pJ} / \mathrm{bit}(\mathrm{ED})$ \\
\hline THO10-OE [49] & UNIS* $^{*}$ & 2010 & PN depletion & MZI & $6 \mathrm{~V} . \mathrm{cm}$ & $N / A$ & $10 \mathrm{G}$ & $6,5 \mathrm{~V}$ & $6 \mathrm{~dB}$ & $15 \mathrm{~dB}$ & $3500 \mu \mathrm{m}$ & $21,1 \mathrm{pJ} / \mathrm{bit}(\mathrm{ED})$ \\
\hline DON10-OL [36] & Kotura & 2010 & PN depletion & Ring & 0,71 V.cm & $\mathrm{N} / \mathrm{A}$ & $12.5 \mathrm{G}$ & $1 \mathrm{~V}$ & $8 \mathrm{~dB}$ & $4 \mathrm{~dB}$ & $12 \mu \mathrm{m}(\mathrm{r})$ & $0,01 \mathrm{pJ} / \mathrm{bit}(\mathrm{AD})$ \\
\hline ZHE10-OE [37] & Luxtera-Sun & 2010 & PN depletion & Ring & $\mathrm{N} / \mathrm{A}$ & $15 \mathrm{GHz}$ & $5 \mathrm{G}(\mathrm{EF})$ & $2 \mathrm{~V}$ & $3 \mathrm{~dB}$ & $6 \mathrm{~dB}$ & $15 \mu \mathrm{m}(r)$ & $0,12 \mathrm{pJ} / \mathrm{bit}(\mathrm{AD})$ \\
\hline GAR11-OE [40] & UNIS* $^{*}$ & 2011 & PN Depletion & MZI & 11 \& 14 V.cm (TE,TM) & $\mathrm{N} / \mathrm{A}$ & $40 \mathrm{G}$ & $6 \mathrm{~V}$ & $6,5 \mathrm{~dB}$ & $15 \mathrm{~dB}$ & $1350 \mu \mathrm{m}$ & $4,5 \mathrm{pJ} / \mathrm{bit}(\mathrm{AD})$ \\
\hline THO11-OE [39] & UNIS* $^{*}$ & 2011 & PN Depletion & MZI & $2,7 \mathrm{~V} . \mathrm{cm}$ & $\mathrm{N} / \mathrm{A}$ & $40 \mathrm{G}$ & $6,5 \mathrm{~V}$ & $3,5 / 10 \mathrm{~dB}$ & $5 \mathrm{~dB} / 15 \mathrm{~dB}$ & $1000 / 3500 \mu \mathrm{m}$ & $5,2 \mathrm{pJ} / \mathrm{bit}(\mathrm{AD})$ \\
\hline 0 & & 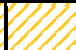 & & & 87 & & & & & & & 80 \\
\hline XU05-NAT [63] & U. Cornell & 2005 & PIN injection & Ring & $\mathrm{N} / \mathrm{A}$ & $\mathrm{N} / \mathrm{A}$ & $1,5 \mathrm{G}$ & $3,3 \mathrm{~V}$ & $6,9 \mathrm{~dB}$ & $N / A$ & $6 \mu \mathrm{m}(\mathrm{r})$ & $\mathrm{N} / \mathrm{A}$ \\
\hline GRE07-OE [34] & IBM & 2007 & PIN injection (PE) & MZI & 0,036 V.cm & $\mathrm{N} / \mathrm{A}$ & $10 \mathrm{G}$ & $7 \mathrm{~V}$ (IPE) & $\mathrm{N} / \mathrm{A}$ & $12 \mathrm{~dB}$ & $200 \mu \mathrm{m}$ & $5 \mathrm{pJ} / \mathrm{bit}(\mathrm{AD})$ \\
\hline XU07-OE [33] & U.Cornell & 2007 & PIN injection (PE) & Ring & $\mathrm{N} / \mathrm{A}$ & $\mathrm{N} / \mathrm{A}$ & $12,5 \mathrm{G}(\mathrm{EF})$ & $8 \mathrm{~V}(\mathrm{IPE})$ & $9 \mathrm{~dB}$ & $\mathrm{~N} / \mathrm{A}$ & $5 \mu \mathrm{m}(r)$ & $0,3 \mathrm{pJ} / \mathrm{bit}(\mathrm{AD})$ \\
\hline MAN07-LEOS [38] & U.Cornell & 2007 & PIN injection (PE) & Ring & $\mathrm{N} / \mathrm{A}$ & $\mathrm{N} / \mathrm{A}$ & $18 \mathrm{G}$ & $6 \mathrm{~V}$ (IPE) & $3 \mathrm{~dB}$ & $N / A$ & $5 \mu \mathrm{m}(r)$ & $N / A$ \\
\hline MAR08-OE[64] & UPS $^{*}$ & 2008 & PIPIN depletion & MZI & $5 \mathrm{~V} . \mathrm{cm}$ & $15 \mathrm{GHz}$ & $\mathrm{N} / \mathrm{A}$ & $\mathrm{N} / \mathrm{A}$ & $\mathrm{N} / \mathrm{A}$ & $5 \mathrm{~dB}$ & $4000 \mu \mathrm{m}$ & $\mathrm{N} / \mathrm{A}$ \\
\hline RAS11-OE[65] & UPS* & 2011 & PIPIN depletion & MZI & $6 \mathrm{~V} . \mathrm{cm}(4 \mathrm{~V})$ & $\mathrm{N} / \mathrm{A}$ & $10 \mathrm{G}$ & $7 \mathrm{~V}$ & $8,1 \mathrm{~dB}$ & $6 \mathrm{~dB}$ & $1800 \mu \mathrm{m}$ & 24,5 pJ/bit (ED) \\
\hline DUS & & $O$ & & & & & & & & & & \\
\hline
\end{tabular}




\begin{tabular}{|c|c|c|c|c|c|c|c|c|c|c|c|c|}
\hline Reference & Group & Year & Electrical structure & Optical structure & Modulation efficiency & EO BW & Bit rate & Vpp & Dyn. ER & Loss & Length & Switching energy \\
\hline & & $\mathbb{Z}$ & & & & & & & & & & \\
\hline DIN10-OE [36] & U. Washington* & 2010 & EO Polymer Pockels & Si slot WG+MZI & 0,8 V.cm (PP) & $3 \mathrm{GHz}$ & $N / A$ & N/A & $N / A$ & $44 \mathrm{~dB}$ (FTF) & $1000 \mu \mathrm{m}$ & $N / A$ \\
\hline WUL10-OL [66] & U. Hamburg & 2010 & EO Polymer Pockels & Slot PhC+MZI & $N / A$ & $40 \mathrm{GHz}$ & N/A & N/A & $N / A$ & $N / A$ & N/A & N/A \\
\hline LIN10-APL [67] & U.Texas & 2010 & EO Polymer Pockels & Slot PhC+MZI & 0,056 V.cm (ng=100) & N/A & N/A & N/A & $\mathrm{N} / \mathrm{A}$ & $45 \mathrm{~dB}$ (FTF) & $308 \mu \mathrm{m}$ & N/A \\
\hline GOU11-OE [68] & U. Washington* & 2011 & EO Polymer Pockels & Si slot WG+ring & N/A & $1 \mathrm{GHz}$ & N/A & $\mathrm{N} / \mathrm{A}$ & $\mathrm{N} / \mathrm{A}$ & N/A & $64 \mu \mathrm{m}(\mathrm{r})$ & $\mathrm{N} / \mathrm{A}$ \\
\hline ALO11-OE [51] & $\mathrm{KIT}^{*}$ & 2011 & EO Polymer Pockels & Si slot WG+Ext MZI & $0,9 . V . c m$ & $>60 \mathrm{GHz}$ & $42,7 \mathrm{G}$ (EF) & $4,1 \mathrm{~V}$ & $N / A$ & $29 \mathrm{~dB}$ & $1700 \mu \mathrm{m}$ & 1,97 pJ/bit (ED) \\
\hline & VII & DIS & 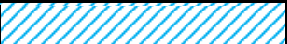 & 0000110 & 7 & 011 & III & WIS & III & 7 & & \\
\hline KUO05-NAT [27] & U.Stanford & 2005 & Ge/SiGe MQWs EA QCSE & Multi-stack WG & $N / A$ & N/A & N/A & $4 \mathrm{~V}$ & $N / A$ & N/A & N/A & $N / A$ \\
\hline LIU08-NATPh [24] & MIT & 2008 & SiGe EA Franz-Keldish & Straight WG & $N / A$ & $1,2 \mathrm{GHz}$ & N/A & $3 \mathrm{~V}$ & $4 \mathrm{~dB}$ & $2 \mathrm{~dB}$ & $50 \mu \mathrm{m}$ & $0,050 \mathrm{pJ} / \mathrm{bit}(\mathrm{AD})$ \\
\hline FEN10-OEGe [25] & Kotura & 2010 & Ge EA Franz-Keldish & Straight WG & $N / A$ & $30 \mathrm{GHz}$ & $12,5 \mathrm{G}$ & $4 \mathrm{~V}$ & $N / A$ & $2,5-5 \mathrm{~dB}$ & $45 \mu \mathrm{m}$ & 0,10 pJ/bit (AD) \\
\hline & & 07 & 6 & & & 111 & & & & & & \\
\hline TAN11-OE [69] & Univ. California & 2011 & InGaAlAs MQWs EA QCSE & MZI & $N / A$ & $42 \mathrm{GHz}$ & $50 \mathrm{G}$ & $2 \mathrm{~V}$ & $9,8 \mathrm{~dB}$ & $5 \mathrm{~dB}$ & $100 \mu \mathrm{m}$ & 0,4 pJ/bit (ED) \\
\hline CHE11-OE [50] & Univ. Californa & 2011 & InAIGaAs MQWs QCSE & Multi-stack WG & 0,24 V.cm & $27 \mathrm{GHz}$ & $40 \mathrm{G}$ & $4 \mathrm{~V}(\mathrm{PP})$ & $11,4 \mathrm{~dB}$ & $1,5 \mathrm{~dB}$ & $500 \mu \mathrm{m}$ & $4 \mathrm{pJ} / \mathrm{bit}$ (ED) \\
\hline 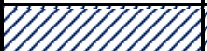 & 771 & DIII & 70111 & 77 & & & & & & & & \\
\hline VLA05-NAT [70] & IBM & 2005 & Thermo-optic & $M Z I+2 D P h c$ & $N / A \quad(n g=10)$ & $10 \mathrm{MHz}$ & $N / A$ & $N / A$ & $N / A$ & $2,5 \mathrm{~dB}$ & $50 \mu \mathrm{m}$ & $N / A$ \\
\hline GU07-APL[43] & U.Texas & 2007 & PIP injection & 2D PhC & $N / A(n g=10)$ & $\mathrm{N} / \mathrm{A}$ & $1 \mathrm{G}$ & 3V (SES) & $3 \mathrm{~dB}$ & $\mathrm{~N} / \mathrm{A}$ & $80 \mu \mathrm{m}$ & $N / A$ \\
\hline CHE09-OL [50] & U.Texas & 2007 & PCP Accumulation & 2D PhC & 0,18 V.cm $(\mathrm{ng}=100)$ & N/A & $1,6 \mathrm{G}$ & $6 \mathrm{~V}$ (SES) & $3 \mathrm{~dB}$ & $40 \mathrm{~dB}$ (FTF) & $300 \mu \mathrm{m}$ & 0,54 pJ/bit (AD) \\
\hline OFA10-IEEE [71] & U. St Andrews & 2010 & Thermo-optic & $M Z I+2 D$ Eng. Phc & $N / A$ & $50 \mathrm{kHz}$ & $N / A$ & $N / A$ & $N / A$ & $8-12 d B$ & $80 \mu \mathrm{m}$ & $N / A$ \\
\hline AKI10-APE [72] & Fujitsu & 2010 & PIN injection (PE) & MZI+ Casc. rings & $N / A$ & N/A & $10 \mathrm{G}$ & 1V & $5 \mathrm{~dB}$ & $N / A$ & $250 \mu \mathrm{m}$ & 0,5 pJ/bit (ED) \\
\hline NGU10-OE [42] & U. Yokohama & 2011 & PN injection (PE) & $M Z I+2 D P h c$ & 0.016 V.cm (ng=18) & $3 \mathrm{GHz}$ & $10 \mathrm{G}$ & $4.2 \mathrm{~V}$ (IPE) & $7,2 \mathrm{~dB}$ & $9,8 \mathrm{~dB}$ & $200 \mu \mathrm{m}$ & 2,9 pJ/bit (AD) \\
\hline BRI11-OE [48] & UPV-UNIS* & 2011 & PN depletion & MZI + Corr WG & 0,45 V.cm (ng=22) & $16 \mathrm{GHz}$ & $40 / 20 \mathrm{G}(\mathrm{EF})$ & $5 \mathrm{~V}$ & $3 / 6,3 \mathrm{~dB}$ & $8 \mathrm{~dB}$ & $500 \mu \mathrm{m}$ & 3,1 pJ/bit (ED) \\
\hline
\end{tabular}

Table 2-2: State-of-the-art of silicon-based modulators

\begin{tabular}{|c|c|c|c|}
\hline (EF): Error free (BER<10-9) & (AD): Available data & (QCSE): Quantum-confined stark effect & (r): radius \\
\hline (IPE): Including pre-emphasis & (ED): Estimated data & (Eng): Engineered & (FTF): Fiber-to-fiber \\
\hline (PE): Pre-emphasis & (PP): Push-pull & (Casc):: Cascaded & (TE, TM):(Transverse electric, Transverse magnetic) \\
\hline (SES): Square electrical signal & (EA): Electro-absorption & (G): Gbits/s & *Device fabrication made at CEA-LETI and/or IMEC \\
\hline
\end{tabular}

Table 2-3: Acronym definitions used in Table 2-2 


\subsubsection{Conclusion and perspectives}

From the previous state-of-the-art analysis, it is clear that optical modulation in silicon is an intensive field of research. As can be observed in Table 2-2, from 2005 (and prior to this date) to the present day, the research effort is mostly concentrated on achieving high speed modulation in pure silicon structures ( $p n$, pin, MOS, pipin...) based on the plasma dispersion effect and to a lesser extent, MQW SiGe and GeSi structures. It is only very recently that high speed modulation has also been reported in hybrid, EO polymer-silicon ( $\mathrm{SOH}$ ) and IIIV-silicon platforms. Although more complex to implement (post-processing required), such highly efficient hybrid structures challenge pure silicon optical modulators as becoming the cornerstone of future enabling technologies. Additionally, numerous type of passively enhancing architectures (rings, cascaded rings, PhCs, LCWG) have been realized since 2005 to enable high speed and efficient optical modulation. While ring resonators have been widely investigated and successfully demonstrated as efficient and low footprint modulators, high speed modulation in slow wave structures such as PhCs and LCWGs, was only reported this year (2011). The last reference [48] stands for the first thorough demonstration of the ground breaking impact of slow light in pure silicon modulators and is precisely the main line of this thesis.

Prior to achieve the latter performance, highly optimized electro-optical modulator designs have been required. Good simulation tools are of paramount importance to predict the device performances. Although current commercial tools efficiently simulate the optical and electrical behavior of almost any given device, the major challenge arises from making them working together in order to provide efficient and systematic simulation procedures. The following section introduces the available optical and electrical simulation tools and their basic principle of operation.

\subsection{Optical Modeling Tools}

The modeling of photonic structures may not always be carried out using approximations in Maxwell's equation. This is especially true when one deals with high index contrast structures with non uniform refractive index profiles all over the propagation length. In that case, Maxwell's equations must be solved in a 
rigorous manner using a full vectorial framework. Several techniques exist to perform these simulations. Among them, we will briefly present those that have been employed to simulate the behaviour of our photonic structures.

\subsubsection{Finite time difference domain (FDTD)}

The FDTD method consists in discretizing Maxwell's equations via finite differences [73]. Basically, the photonic structure to be computed is subdivided into a mesh with (as high as possible) predefined resolution. The advantage of such a method is that it enables the computation and monitoring of the electric and magnetic fields propagating through any type of photonic structure geometry over space and time. However, as mentioned earlier, although this method provides a flexible framework, the high resolution and hence tight mesh required to achieve accurate results leads to time and resource consuming simulations. This issue limits the applicability of such a method to relatively short field propagation length. Alternatively, the 2D FDTD effective index method may alleviate the computational time while providing to some extent fairly accurate results. A common FDTD software package is FULLWAVE TM [74].

\subsubsection{Plane wave expansion (PWE)}

In contrast with FDTD, the plane wave expansion method computes the electromagnetic field as a superposition of planes waves, which are straightforward solutions of Maxwell's equations in an uniform medium. More precisely, the electric and magnetic fields are taken as time-independent solutions of a "master" equation in the frequency domain, leading hence to a relatively simple eigen-value problem. The solutions of this equation are eigen modes that are simply linear combinations of planes waves (which form a basis). The more planes waves, the more accurate the results. This method is particularly interesting due to the fact that the localized electromagnetic modes in periodically shaped structures such as PhCs may be written as Bloch states (as a result of the discrete translational symmetry in the corresponding direction). Because of this invariance, a unit cell, which will be repeated infinitely over a given period in the 3 dimensions (or 2 if the structure is an infinitely tall $\mathrm{PhC}$ ), is to be defined. The method accuracy is highly dependent upon the number of plane waves used for the simulations and the cell size, which again sets a trade-off between computational time and precision. When the unit cell is large, it is called a super- 
cell just as in line defect PhCs. More details and concrete examples are provided in the following sections. The simulations results of this work were performed using an user-friendly software package BANDSOLVE TM [74].

\subsubsection{Beam propagation method (BPM)}

For some structures, which are uniform in the propagation direction, on may use the BPM method. It is basically based on the parabolic approximation of the Helmoltz Equation. Just as for the FDTD, this method uses finite-differences that lead to a discretized structure within a finite computational domain. Using a BPM-based mode solver may be particularly useful to compute the effective indices of a given optical mode and quickly evaluate its electric and magnetic field profiles as its amount of confinement in an uniform structure along the propagation direction. However, the BPM method features a series of restrictions that prevent from solving periodically index perturbed waveguides, and may suffer from a lack of accuracy in simulating high index contrast and multimode structures. These restrictions may be partially removed via additional algorithms such as those implemented in the commercial software package BeamPROP тм [74].

\subsection{Electrical Modeling Tools}

\subsubsection{Semiconductor device simulation framework: ATLAS}

ATLAS $\mathrm{TM}$ is a physically-based $2 \mathrm{D}$ and $3 \mathrm{D}$ device simulator. It predicts the electrical behavior of specified semiconductor structures and provides insight on the internal physical mechanisms associated with device operation. DC, AC and transient behavior of a given device may be modeled via solving the discretized Poisson's equation (derived from Maxwell's law), which basically relates variations in electrostatic potential to local charge densities. The continuity and the transport equations describe the way the electron and hole densities evolve as a result of transport, generation, and recombination processes. ATLAS TM consists of sub-modules that are particularly suitable for any specific electrical device modeling. As an example, S-Spices efficiently simulates the characteristics of silicon-based semiconductor devices including MOS, bipolar, SOI device technologies etc... This relevant tool provides accurate and visual insights of complex silicon-based devices. 


\subsection{Electro-Optical Modeling Convergence}

As the name suggests, electro-optic modulators consist of both an electrical and optical parts. Therefore, device modeling must be realized in both electrical and optical domains. As mentioned previously, ATLAS TM is particularly efficient in simulating any type of semiconductor structure while both BeamPROP's TM mode solver and BANSOLVE's ${ }^{\text {TM }}$ PWE algorithm provide a good platform to simulate respectively the modal effective indices of uniform photonic structures and the propagation characteristics of periodic waveguides. In other words, taken individually, these softwares can efficiently simulate the properties of a given electro-optical device. However, the issue is bridging the simulation results provided by those simulators, which were not initially designed to work together.

\subsubsection{From ATLAS to BPM}

The BPM mode solver provides a suitable and quick tool to readily compute the modal effective indices of simple rib (resp. strip) waveguide geometries in a fullvectorial environment. An effective index value for a given propagating mode is just what we need to evaluate the influence of external electrical perturbations. As seen before, any change in free carrier (electrons and holes) concentration leads to a local change of the refractive index of the material. Consequently, the trick consists in including the calculated refractive index distribution over the entire area of the waveguiding structure. Because we assume that the refractive index change will be uniform all over the propagation length, we can hence calculate the resultant modal effective index caused by the overlap between the free carriers (converted into local refractive index perturbations) and optical mode propagating through the altered medium. Since that for a given electrical structure the intensity of the electrical perturbation is related to the applied electric field, the modal effective index variations, which the overall modulation parameters derives from, can be estimated. The combination of these two tools is particularly suitable to simulate the operation of rib waveguide-based electrooptical modulators. An example of an optical mode propagating through a rib waveguide and overlapping with a given refractive index distribution is illustrated in Figure 2-11. 


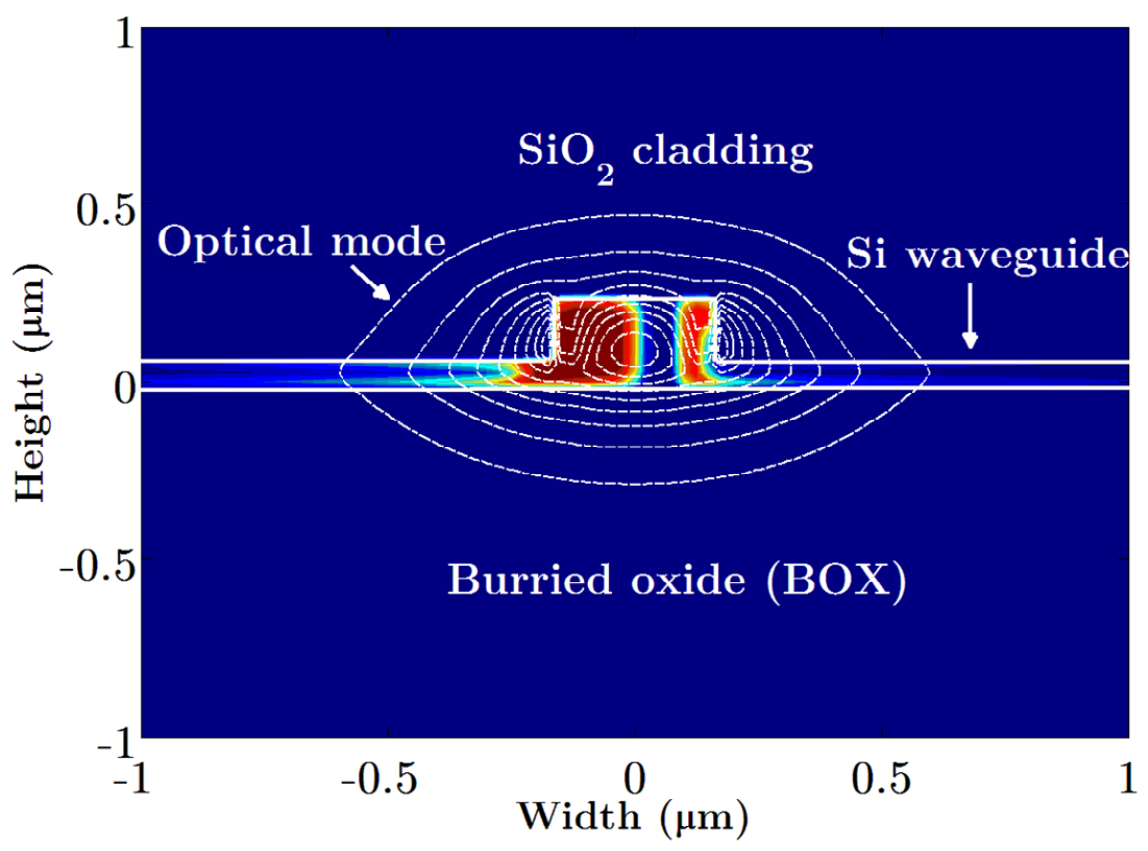

Figure 2-11: White dashed contour lines depict an optical mode profile overlapping with a refractive index change distribution achieved via depletion of a $p n$ junction in a submicrometer rib waveguide. The mode was calculated with Beamprop ${ }^{\mathrm{TM}}$ and the real (resp. imaginary) refractive index change extracted via Soref's Equations (1.63) and (1.64) from the free carrier densities, itselves simulated with ATLASTM.

\subsubsection{From ATLAS to PWE}

When the optical waveguide is no longer uniform over the propagation direction but rather periodic for instance, a PWE-based algorithm is required. Just as for combined ATLAS-BPM simulations, the change in refractive index distribution arising from the variation in free carrier concentration is included into one unit cell, taking into account the differences in shape. Because of this, additional 2D ATLAS simulations corresponding to the different photonic structure sections may be required. An example of a $1 \mathrm{D}$ periodic structure is provided in Figure 2-12. PWE simulations iterations will then be realized for varying free carrier concentration converted via an "in-house" code into a refractive index distribution, which will in turn alter the effective index of the optical mode. This procedure has been followed to simulate the performance of the silicon EO modulators presented in this thesis. The full conversion code is provided in appendix A. 


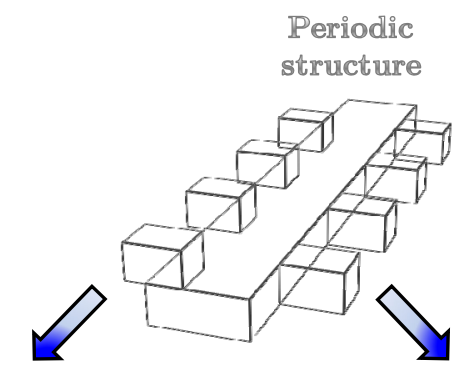

Thin section

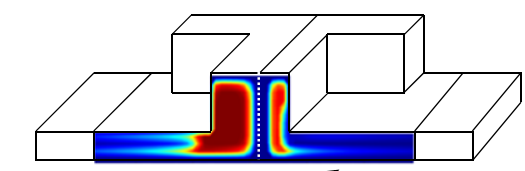

П \ Refractive index. distributions
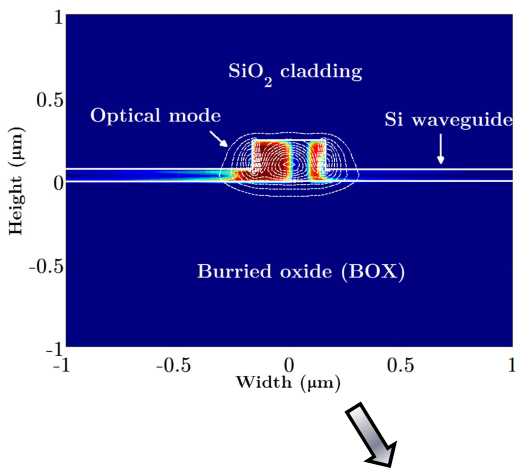

Wide section
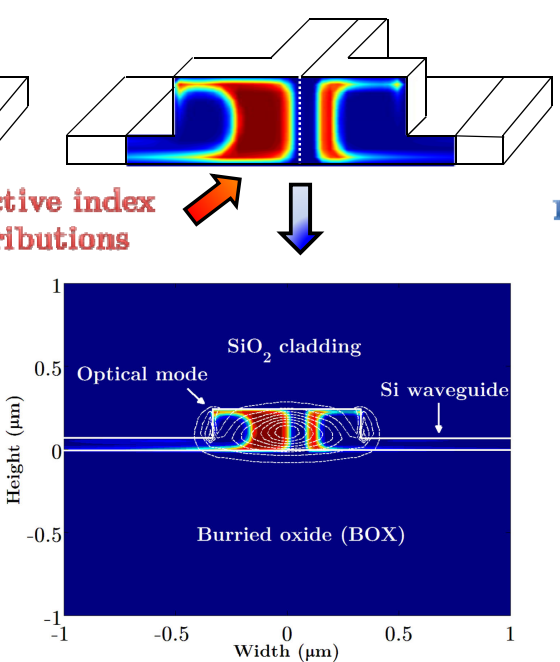

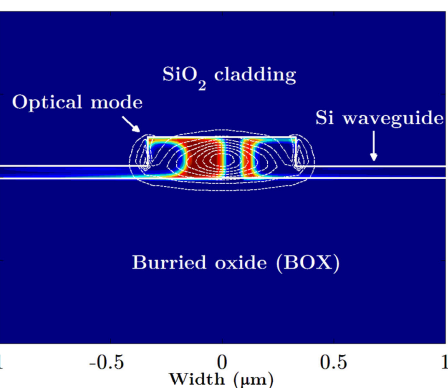

$\sqrt{2}$

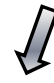

(a)

BANDSOLVE (PWE) 3D PASSIVE DESIGN (b)

ATLAS:

2D FREL CARRTER. DENSTTTES

$\checkmark$

REFRACTIVE INDEX DISTRIBUTIONS ("IN HOUSE" CONVERSION)

(c)

BANSOLVE+ATLAS 3D ACTIVE DESIGN

(d)

ELECTRO-OPTICAL DEVICE PARAMETERS (MODULATOR)

Figure 2-12: Overview of the procedure to simulate electro-optical modulators based on active periodic structure. (a) 3D passive design using PWE algorithm, (a) 2D Electrons and hole densities are converted via a in "in-house" code into refractive index distributions via Soref's Equations (1.63) and (1.64) (c) This densities are included back into the 3D PWE simulator to calculate the (d) modal effective index change for varying refractive index distribution.

\subsubsection{Conclusion}

In conclusion, we have given an overview of the simulations techniques and procedures that have been used to predict the behavior of the EO modulators presented in this thesis. We have mentioned the use of a conversion code that bridges the electrical and optical domain via converting free carrier densities into a non-uniform refractive index distribution, which may be incorporated back into the PWE simulator in order to compute the altered modal effective index and related electro-optical parameters. 


\subsection{Passive and Active Characterization Setups}

\subsubsection{Introduction}

In the previous chapter, we have given an overview of some of the simulations techniques employed to efficiently predict the behavior of EO modulators. However, it is equally important to describe the experimental setups used for characterizing the fabricated devices. Two main types of passive characterization setups, which are widely used for passive silicon photonic chip characterization, are described hereafter. Active experimental setups for static and high speed characterization of EO modulators are also described further on.

\subsubsection{Horizontal coupling versus vertical coupling}

The first issue that "silicon photonicists" have to deal with is coupling light coming from a standard single mode optical fiber (SMOF) with $8 \mu$ m-core diameter to a silicon waveguide having sub-micrometer dimensions in an efficient manner. Putting the fiber in close contact with the waveguide will obviously result in highly inefficient coupling as only a small fraction of light will make its way from the cylinder-shaped fiber to the rectangular waveguide. To solve this problem, intensive research has been carried out and different kinds of coupling techniques have been demonstrated. Basically, we may divide them in two main families: Horizontal (butt- or end-fire coupling) or vertical coupling configurations via grating couplers as described in Figure 2-13. Although their description is beyond the scope of this thesis, the highest coupling efficiencies reported so far reaches values as high as $80 \%(\sim 1 \mathrm{~dB}$ coupling loss per facet) $[75,76]$ and $70 \%$ ( 1.5 dB coupling loss per grating) [77, 78] respectively for inverted tapers and grating couplers. However, these are the very optimized cases which usually trade performance for more complex fabrication process. For "mass testing" purposes, i.e. when only fair coupling efficiency is required, current silicon photonic fabrication platforms offer standard gratings couplers with an average $30 \%$ efficiency with SMOFs with $8 \mu \mathrm{m}$-core diameter $(\sim 5.2 \mathrm{~dB}$ coupling loss per grating). In the case of butt-coupling, if no inverted tapers are used, the coupling efficiency may be improved via the use of lensed fiber with 2.5 um mode field diameter (MFD) coupled to a silicon waveguide of similar width. This technique exhibit typically $15 \%$ to $20 \%$ efficiency ( 8 to $7 \mathrm{~dB}$ coupling loss per facet) but is highly dependent upon the facet quality (an anti reflection coating or facet 
polishing may help improve these values). Additionally, coupling interface-fiber alignment tolerance is usually higher for vertical coupling schemes (typically \pm 1 $\mu \mathrm{m})$ than for horizontal ones $( \pm 0.3-0.4 \mu \mathrm{m})$.

(a)

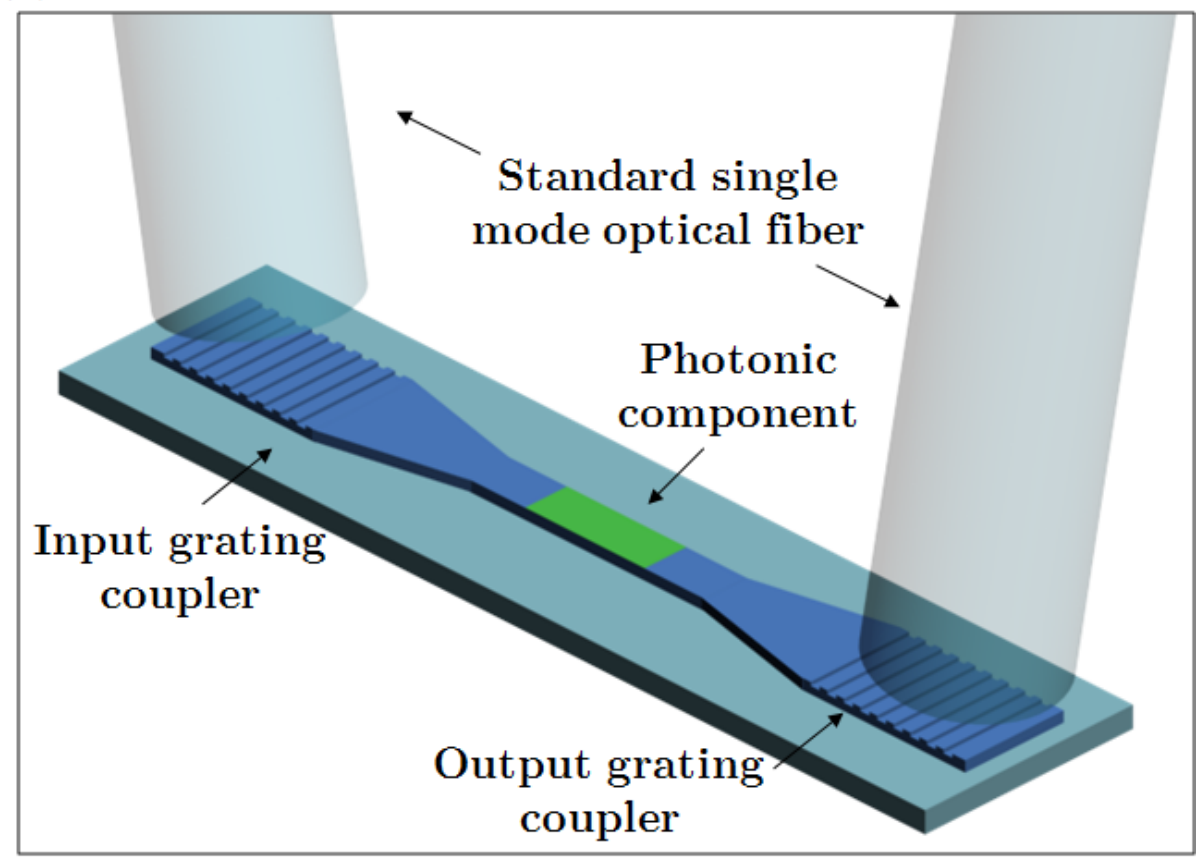

(b)

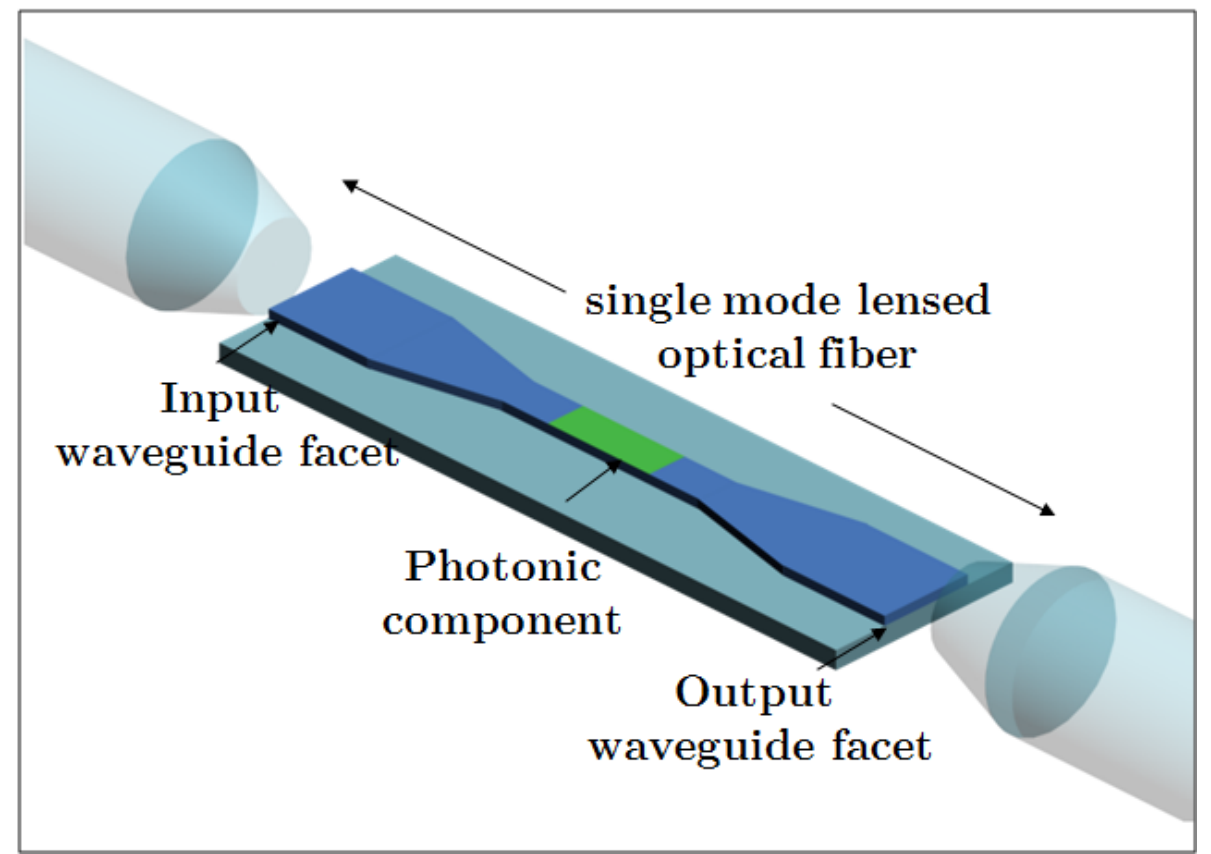

Figure 2-13: "Standard" fiber-to-the-chip coupling techniques. (a) Vertical coupling schemes via grating couplers (b) Horizontal (butt) coupling scheme 


\subsubsection{Small signal characterization}

Small signal measurements allow for the determination of the electrical and electro-optical frequency response of a given electrical or electro-optical device via the calculation of the scattering parameters.

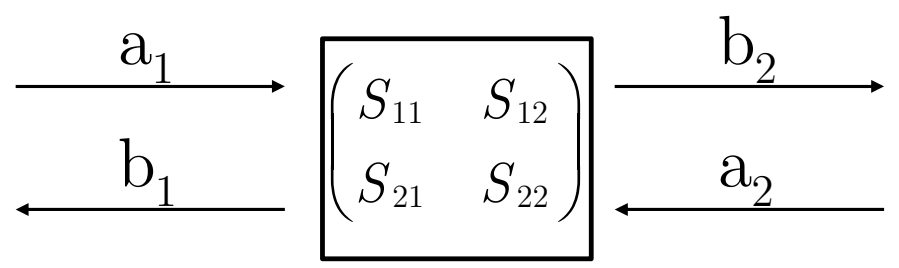

Figure 2-14: Schematic description of a two port network

For a two port-network, as described in Figure 2-14, the S-matrix, which relates the incident and reflected waves through the system, is given by:

$$
\left(\begin{array}{l}
b_{1} \\
b_{2}
\end{array}\right)=\left(\begin{array}{ll}
S_{11} & S_{12} \\
S_{21} & S_{22}
\end{array}\right)\left(\begin{array}{l}
a_{1} \\
a_{2}
\end{array}\right)
$$

Where $a_{i}$ and $b_{i}$ denotes respectively the voltage incident at and leaving each port " $i$ ". In a $50 \Omega$ matched system, i.e. when the electrical transmission line is terminated by a $50 \Omega$ resistance, the transmitted RF voltages will be completely absorbed and no reflections will take place. Hence, if $a_{2}=0$ or $a_{1}=0$ depending on which port the load is placed, and from matrix (1.66) the scattering coefficients may be written as:

$$
\begin{aligned}
& a_{2}=0 \Rightarrow S_{11}=\frac{b_{1}}{a_{1}} \quad \text { and } \quad S_{21}=\frac{b_{2}}{a_{1}} \\
& a_{1}=0 \Rightarrow S_{22}=\frac{b_{2}}{a_{2}} \quad \text { and } \quad S_{12}=\frac{b_{1}}{a_{2}}
\end{aligned}
$$

These scattering coefficients may be represented either in linear magnitude or in decibels $(\mathrm{dB})$ as:

$$
S_{i j}(d B)=20 \log _{10}\left[S_{i j}(\text { linear magnitude })\right]
$$

As we will see in Chapter 3, the EO modulator have a $50 \Omega$ impedance matched travelling wave configuration. Therefore, we are particularly interested in 
evaluating on one hand, the $S_{11}$ and $S_{21}$ electrical parameters to estimate the corresponding reflections and RF losses along the transmission line and, on the other hand, the $S_{21}$ electro-optical parameter to estimate the 3dB roll-off bandwidth of our modulator or device under test (DUT).

Figure 2-15 describes the generic setup used to estimate $S_{21}$ electro-optical parameter magnitude. The equipment models are given in Chapter 3. Transverse electric (TE) polarized light emitted by an external cavity laser (ECL) is injected into the modulator via grating couplers. The transmitted power is optimized through the use of a polarization controller (PC). The small electrical signal delivered by the port 1 of the RF vectorial network analyzer (VNA) is coupled a DC bias via a bias-Tee and applied through high speed ground-signal-ground (GSG) probes to the travelling wave electrodes terminated externally by a $50 \Omega$ load coupled to a DC block. The output modulated signal is amplified with an erbium-doped fiber amplifier (EDFA) and photodetected by a high speed photodiode connected to the port 2 of the RF VNA in order to extract the electro-optical S21 transfer function. It is worth noting that the setup calibration is performed using an impedance standard substrate. For completeness, pictures of the entire setup are also shown in Figure 2-16.

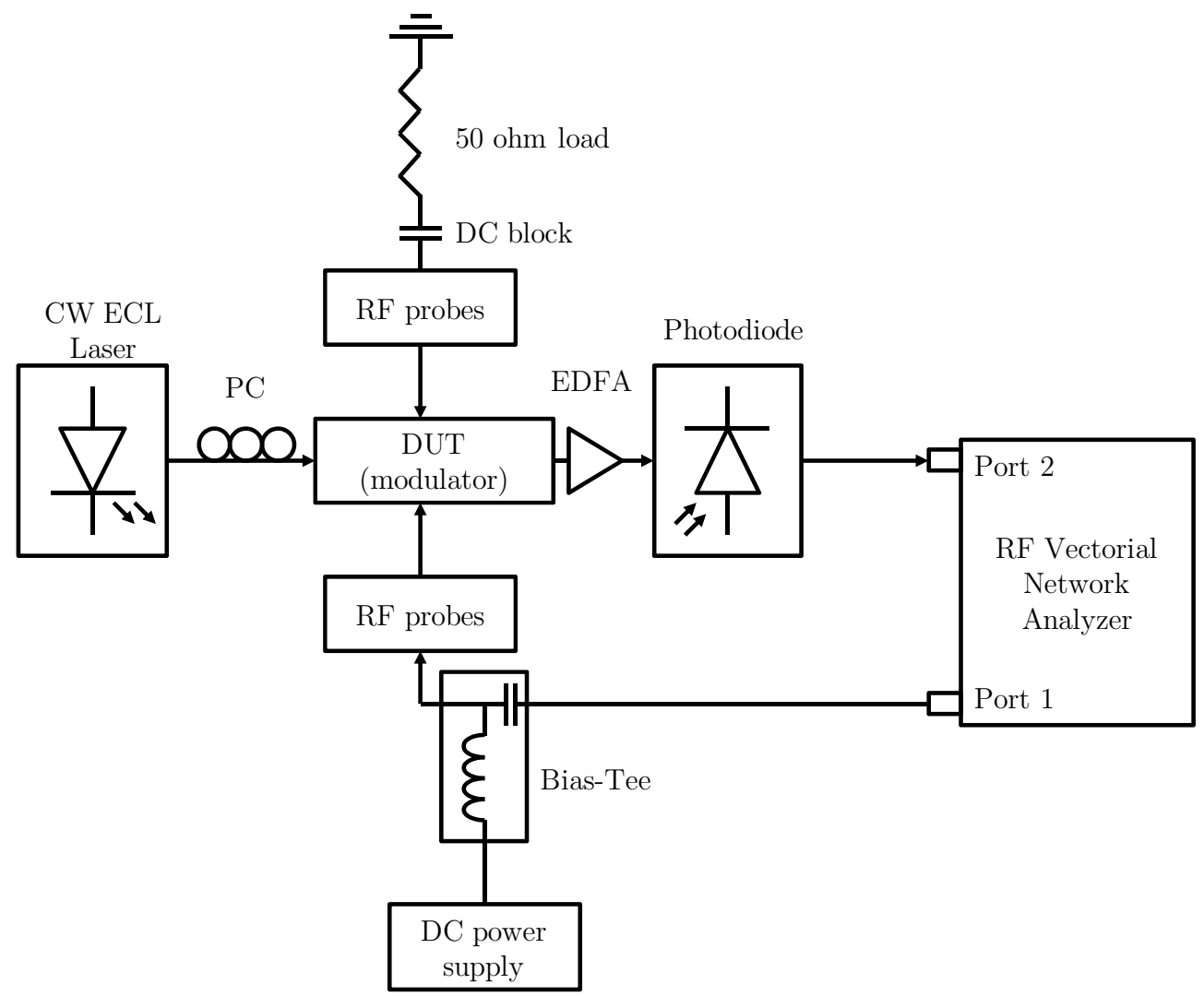

Figure 2-15: Schematic of the characterization setup to determine the S21 electro-optical response 


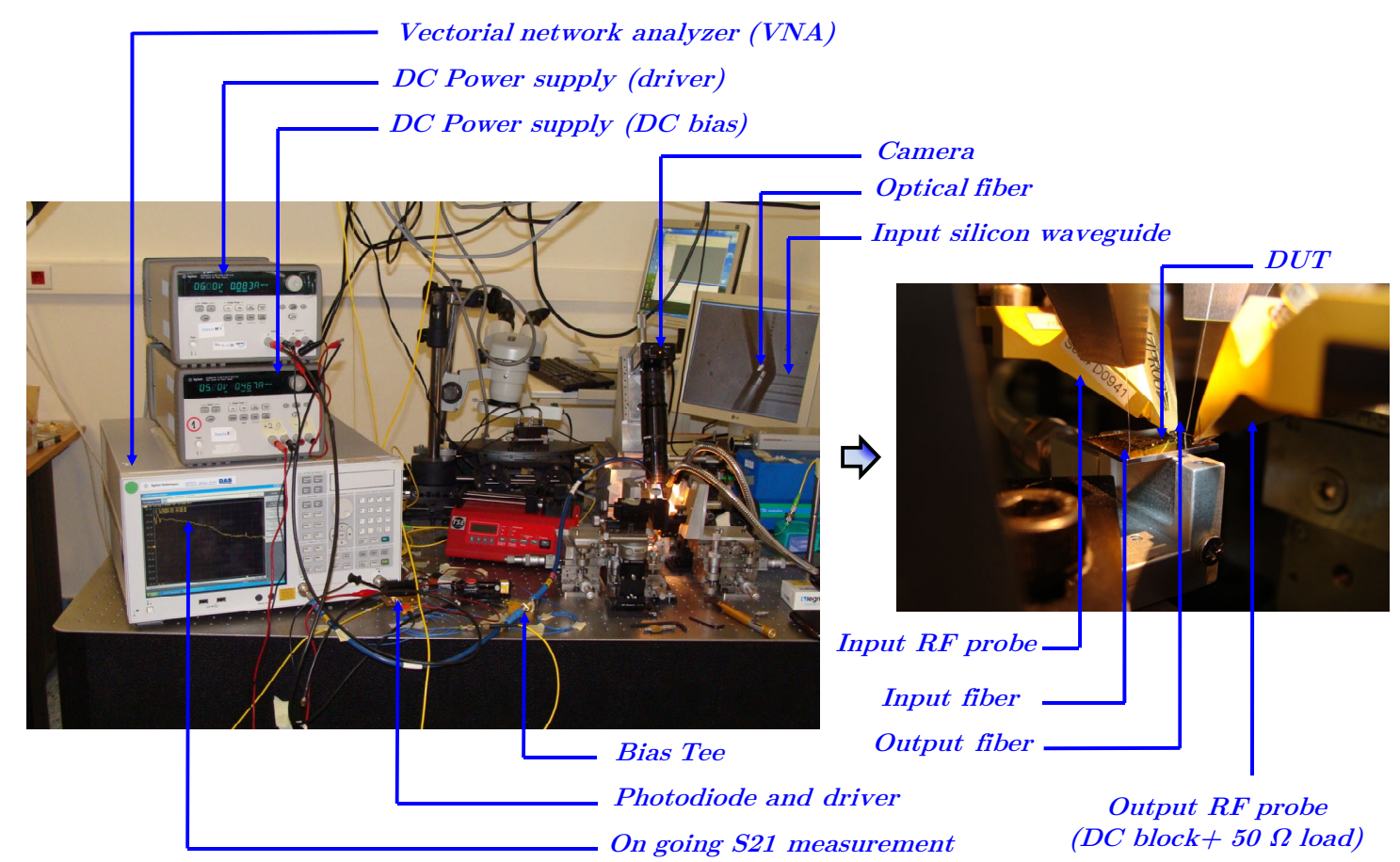

Figure 2-16: Pictures of the characterization setup to determine the S21 electro-optical response

\subsubsection{Data transmission and bit error rate measurements}

The electro-optical $S_{21}$ provides a fairly accurate indication on the maximum data transmission rate that a given electro-optical device may support without degrading the optical signal integrity through the analysis of the resultant eye diagram (see section 2.1.2). The description of the generic setup is given in Figure 2-17 below.

Data transmission measurements are realized by driving the modulator with a non-return to zero (NRZ) pseudo-random bit sequence (PRBS) of length $2^{31}-1$ delivered by a bit pattern generator (BPG) connected to an external clock. The electrical signal is generally amplified through a high speed RF amplifier to achieve a decent voltage swing $\left(V_{p p}\right)$ and combined to a DC bias using a bias-tee. For consistency with $\mathrm{S}_{21}$ electro-optical measurements, the modulating signal is applied to the travelling wave electrodes, which are terminated externally by a 50 $\Omega$ resistance coupled to a DC block. The output modulated optical signal is then filtered via and optical filter $(\mathrm{OF})$, split equally $(-3 \mathrm{~dB})$ and photodetected by a digital communication analyzer (DCA). The BER is measured together with the eye diagram using an error analyzer (EA) and evaluated at the photodetector as a 
function of the variation in optical power using a variable optical attenuator (VOA). Pictures of the entire setup are also shown in Figure 2-18.

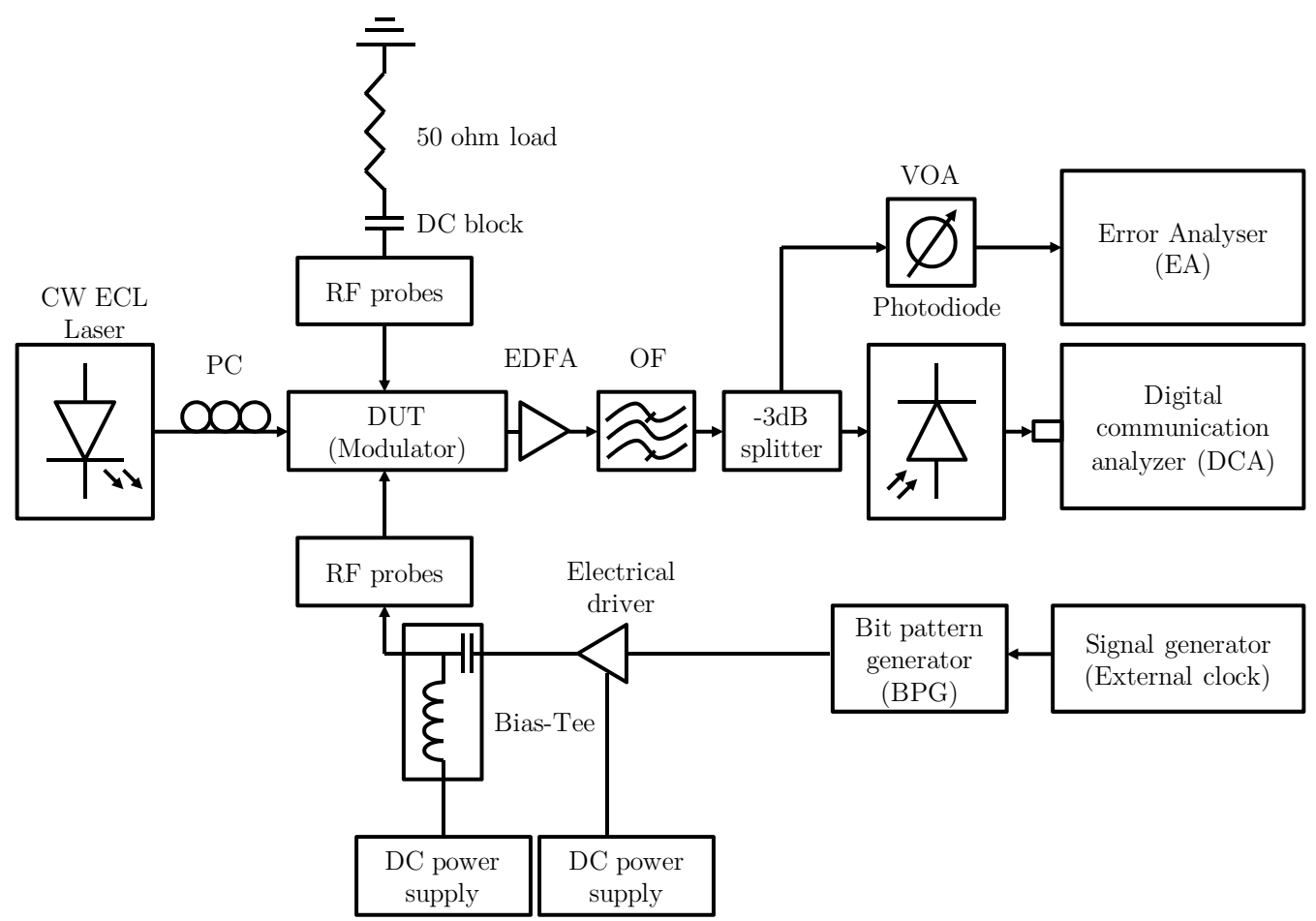

Figure 2-17: Schematics of the characterization setup for eye diagram acquisition and BER measurements

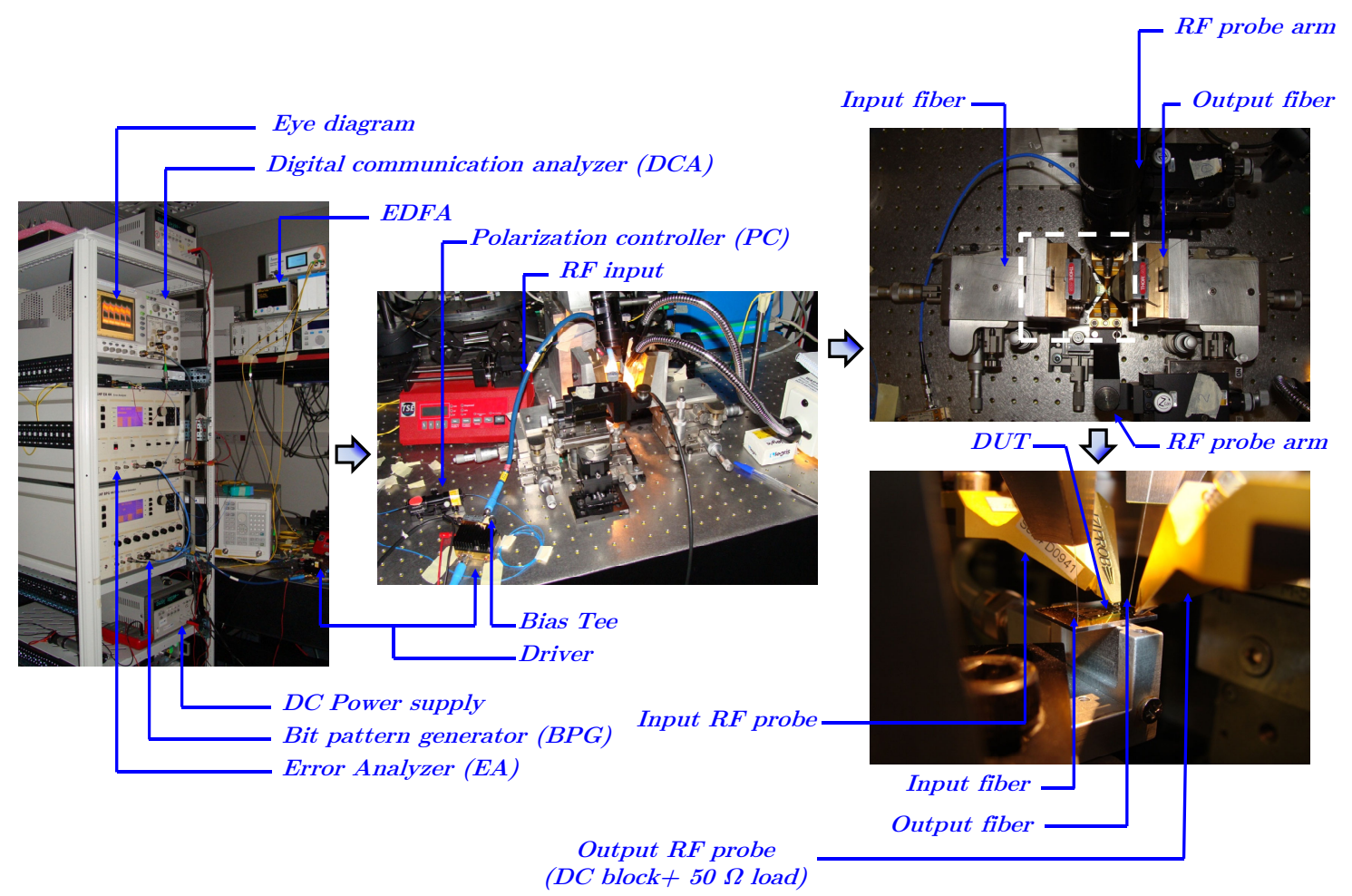

Figure 2-18: Pictures of the characterization setup for eye diagram acquisition and BER measurements 


\section{Chapter 3}

\section{Compact Depletion-Based Slow Wave Modulator}

This chapter is devoted to the design, fabrication and characterization of an ultrafast and compact plasma dispersion-based silicon electro-optical modulator, which combines the attractive properties of slow light propagation in a nanostructured periodic waveguide together with a high speed semiconductor pn diode. The resulting device feature high modulation efficiency and is entirely CMOS compatible.

\subsection{Motivation and Background}

As exposed in the preceding sections, the progress towards high speed modulation in silicon has experienced a dramatic increase in the last 5 years, pushing for the first time the bandwidth of modulators to up to $30 \mathrm{GHz}$ with a record transmission rate of $40 \mathrm{Gbit} / \mathrm{s}$ [79] in a depletion type MZI. However, this stateof-the-art performance in terms of speed has been achieved at the expense of a low extinction ratio of approximately $1 \mathrm{~dB}$. The reasons that may be put forward for such low extinction ratio are on one hand, the long interaction length required to achieve a $\pi$-phase shift and on the other hand, the impedance mismatch between the modulator and driver. The figure of merit $V_{\pi} L_{\pi}=4 \mathrm{~V} . \mathrm{cm}$ suggests that for $V_{p p}=6 \mathrm{~V}$ applied voltage over a $1 \mathrm{~mm}$ long phase shifter, the achieved phase shift would be $\Delta \Phi=0.15 \pi$, meaning incomplete modulation. In addition, the impedance mismatch between the driver and modulator at high frequency causes a portion of the drive voltage to be lost, thus leading to lower modulation efficiency than expected. Recently, an equivalent transmission rate of $40 \mathrm{Gbit} / \mathrm{s}$ 
was reported using both compact (1mm long) and large footprint (3.5mm long) Mach-Zehnder interferometer (MZI) modulators based on carrier depletion operation [39]. However, for the compact version of the device, the state-of-the-art bit rate performance was achieved with a lower ER of $3.5 \mathrm{~dB}$, making error free modulation essentially unviable.

These examples well illustrates the common issues encountered by depletion silicon modulator designers. First, the relatively low refractive index change produced by the plasma dispersion effect in a reverse biased $p n$ diode gives rise to interaction lengths on the order of several millimeters in order achieve a $\pi$-phase shift. Therefore, within the current state of knowledge on silicon modulators, the trade-off between phase shifter compactness and achievable phase shift naturally arises. Apart from the large footprint, the choice of a long phase shifter may be impaired by the loss of the CPWG transmission line signal limiting the voltage effectively applied to the phase shifter under high speed operation and the higher influence of the velocity mismatch between the co-propagating electrical and optical signals (where appropriate). The latter issues may be partially overcome by opting for shorter phase shifter with the same electrical design but at the expense of a lower phase shift.

Being aware of the abovementioned issues, the realization of silicon modulators featuring simultaneously high bandwidth, high ER, low loss and short interaction length appears to be challenging. This is the reason why in this chapter, we propose a technique to increase the interaction between the depleted free carriers and optical mode by forcing light to travel over a longer optical path while keeping the optical structure as small as possible. This apparent paradox can readily be solved using slow wave periodic structures which feature a repeated pattern all over the propagation region. Although the idea of enhancing the sensitivity of active silicon devices exploiting $\mathrm{PhCs}$ was initially proposed nearly 10 years ago [80], there has been no clear experimental evidence of a really efficient silicon optical modulator based on the plasma dispersion effect in slow wave periodic structures. The idea of using 1D slow wave structure to achieve high speed modulation over a short length has been theoretically investigated, proposed and accepted within the FP7-HELIOS project, offering a suitable platform to complete the fabrication of such a novel silicon modulator. 


\subsection{Target Applications of Slow Wave Structures}

The attractive features of the slow wave phenomenon have spurred researchers to seek promising application fields. Slow light "on-chip", usually produced in periodic dielectric structures, has very attractive properties since it allows a deep enhancement of light-matter interactions. This is of great interest for silicon, a material not initially destined to exhibit active optical functionalities. Though passive PhCs have been extensively studied, there is relatively little literature on slow wave-based active devices. Therefore, we gathered the available bibliography on experimentally demonstrated slow wave enhanced devices and identified four main sectors of application: access network, optical buffering, all-optical signal processing and sensing.

For access networks, the slow wave phenomenon has been proposed and used as a mean to enhance the efficiency of electro-optic modulators based on the plasma dispersion effect [45] as well as that of thermo-optic modulators [70]. Indeed, electro-optic modulation in a line defect $2 \mathrm{D}$ PhC has been shown to be viable. The resulting device features a very high figure of merit of 0.18 V.cm, meaning that for a $6 \mathrm{~V}$ applied voltage, a pi-phase shift is achieved over a length of $300 \mu \mathrm{m}$. This reduced interaction length is approximately one order of magnitude lower than for conventional rib waveguide electro-optical modulators (rib waveguide EOM). Furthermore, the modulator was driven under a square electrical signal at $1.6 \mathrm{Gbits} / \mathrm{s}$ and shown $3 \mathrm{~dB}$ extinction ratio. In addition, the low capacitance of the device also allowed for an energy per bit consumption as low as $0.54 \mathrm{pJ} /$ bit at the expense of lower operating bandwidth and higher losses. Vlasov et al. [70] shown also a dramatic slow wave enhancement in a line defect $2 \mathrm{D} \mathrm{PhC}$ via thermo-optic (TO) effect. The group index is controlled externally varying the electrical current flowing through the heating pads. The resulting device features a small footprint $\left(400 \mu \mathrm{m}^{2}\right)$, low operating power at the expense of a lower operating speed. Numerous applications are foreseen for this device, including enhanced TO modulators, tunable time delay lines and dynamic dispersion compensators.

Optical buffering is also a key application field of the slow wave phenomenon as demonstrated by Xia et al. [81] Their device consists of 100 cascaded ring resonators with $6.5 \mu \mathrm{m}$ radius. The total group delay (510 ps) is given by the sum of the delay introduced by each ring resonator. Furthermore, the footprint of such delay line $\left(9000 \mu^{2}\right)$ is considerably smaller than in the 
case of a simple $4 \mathrm{~cm}$ long bent photonic wire, increasing therefore the total achievable delay per unit area. This enhanced device footprint is at the expense of the three times higher insertion losses and reduced bandwidth compared to bent photonic wires.

All-optical processing is also pointed as an alternative to electro-optical control of light. Slow waves actually help increase the interaction between the propagating light and the non-linear material as demonstrated by Monat et al [82] through strongly enhanced third harmonic generation and self phase modulation in a line defect $2 \mathrm{D} \mathrm{PhC}$. As a result, the peak pump power to generate such a non-linear effect is significantly reduced to a few watts. Engineered $\mathrm{PhCs}$ were employed to avoid counterproductive dispersion effects [83]

Finally, using PhCs as sensors has become a trend in sensing applications. The sensing techniques are either based on the shift produced by the refractive index change associated with the introduction of a liquid containing proteins, molecules, or DNA on either the photonic band gap $[84,85]$ or the position of the Fabry-Pérot fringes in the slow light regime [86].

\begin{tabular}{|c|c|c|}
\hline Applications & Slow-wave exploitation & Example \\
\hline \multirow[b]{2}{*}{ Access network } & $\begin{array}{c}\text { Enhanced efficiency in EO } \\
\text { modulation }\end{array}$ & $\begin{array}{c}\text { Very low } \mathrm{V}_{\pi} \mathrm{L}_{\pi} \mathrm{PhC} \\
\text { waveguide modulator }[45]\end{array}$ \\
\hline & $\begin{array}{l}\text { Enhanced efficiency in TO } \\
\text { modulation, tunable time delay } \\
\text { lines, dynamic dispersion } \\
\text { compensators }\end{array}$ & $\begin{array}{c}\text { Compact and low power } \mathrm{PhC} \\
\text { TO modulator }[70]\end{array}$ \\
\hline $\begin{array}{l}\text { Optical } \\
\text { buffering }\end{array}$ & $\begin{array}{c}\text { Enhanced and tunable group delay } \\
\text { in optical delay line }\end{array}$ & $\begin{array}{l}\text { Compact and low power } \mathrm{PhC} \\
\text { TO modulator [70]and } \\
\text { ultracompact optical buffering } \\
\text { with rings [81] }\end{array}$ \\
\hline $\begin{array}{l}\text { All-optical } \\
\text { signal } \\
\text { processing }\end{array}$ & Enhanced nonlinear effects & $\begin{array}{l}\text { Slow-light enhanced nonlinear } \\
\text { optics in } \mathrm{PhC}[83]\end{array}$ \\
\hline Sensors & Enhanced light-matter interaction & Enhanced bio-sensing[84-86] \\
\hline
\end{tabular}

Table 3-1: target applications of slow light-based devices and corresponding experimental realizations 
In conclusion, the slow wave phenomenon has confirmed researcher's expectations in terms of light-matter interaction enhancement by showing interesting advances in the field. This attractive enhancement is often related to higher losses and lower bandwidth. However, engineered PhCs shown that this does not hold as a general rule. More applications should emerge in the future. The main target applications of the slow wave phenomenon are summarized in Table 3-1.

\subsection{Optical Design}

\subsubsection{Slow light propagation background}

The group velocity of light, defined by $v_{g}=c / n_{g}$, where $n_{g}$ is the group index, provides an estimate on how much the group velocity of a wavepacket propagating through a dispersive material is reduced compared to the speed of light in a vacuum $c$. The slow light phenomenon was first and foremost shown in atomic media by electromagnetically induced transparency [87], in semiconductors exhibiting population oscillations [88], as well as by excitation of acoustic waves in fibers [89]. Likewise, silicon-on-insulator (SOI) technology provides an effective platform to produce slow light "on-chip" in 1, 2 or even 3 dimensional periodic structures, where the dielectric periodicity is usually achieved via ordered nanostructuring [70, 90-92] as shown in Figure 3-1.

(a)

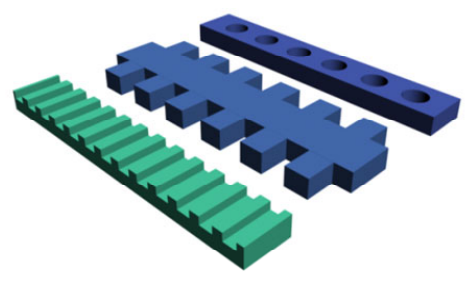

(b)

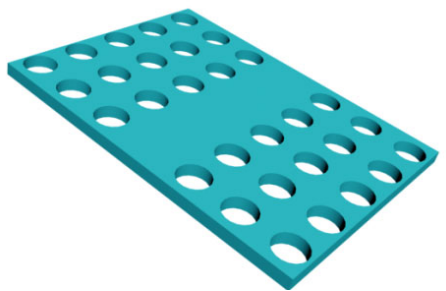

(c)

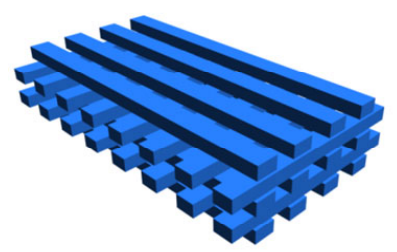

Figure 3-1: (a) one dimensional PhCs. (b) two dimensional $\mathrm{PhC}$ with line defect. (c) three dimensional « wood pile» $\mathrm{PhC}$

The period, size and shape of the repeated pattern define the frequency at which the speed of light theoretically drops inside the waveguide, thus providing complete liberty for the designer. The nature of slow light, which consists in the coupling in phase and amplitude between successive back and forth reflections experienced by the propagating wave at each unit cell, can intuitively be described with the ray optics theory in mind. Here, we focus on a 1 dimensional 
(1D) laterally corrugated periodic structure (LCWG), although the slow wave propagation principle remains the same for the other types of $\mathrm{PhCs}$. The optical properties of such waveguide can readily be modeled under a 3 dimensional PWE environment in a fairly short time. A typical band diagram of a deep-etched LCWG is depicted in Figure 3-2 (a). Here, we describe two slow light propagation mechanisms by either (b) omnidirectional reflection or (c) backscattering.

(a)

(b)

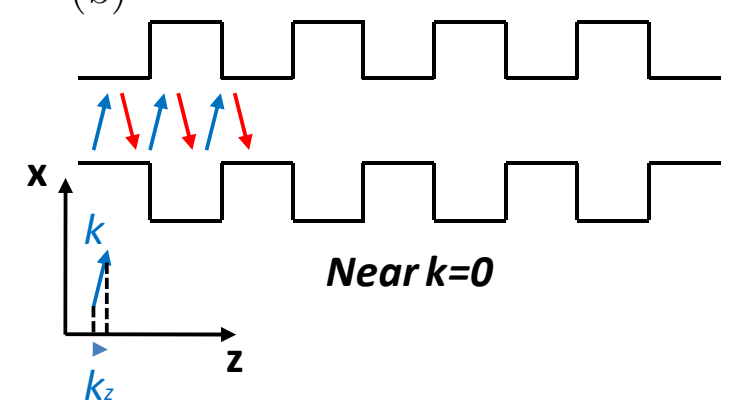

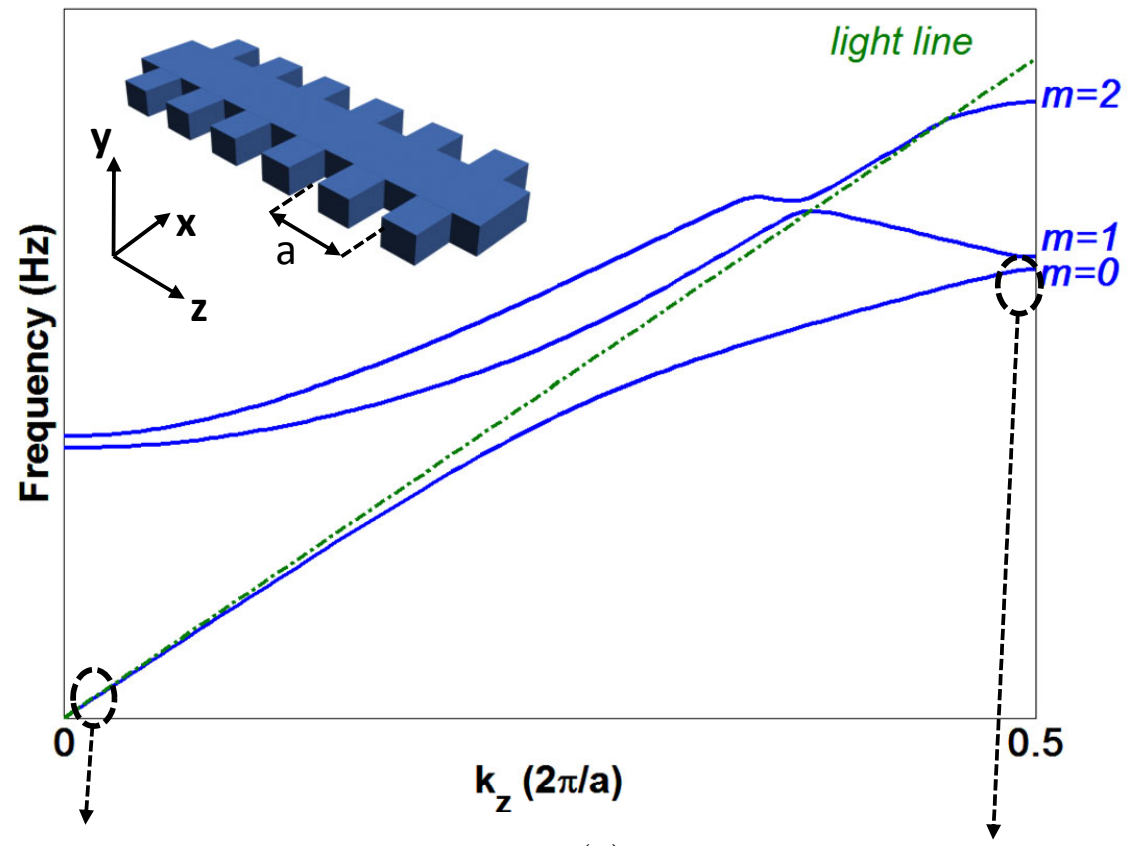

(c)

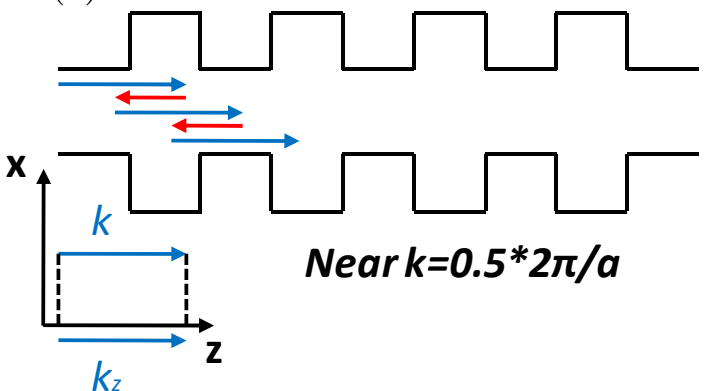

Figure 3-2: (a) Band diagram of a LCWG. The blue solid lines represent the first three eigen odd modes with respect to the plane of symmetry of the structure $\mathrm{X}=0$ as explained further in section 3.3.2. Only those located below the light line (green dashed) propagate through the optical structure theoretically without losses. (b) Slow light propagation by omnidirectional reflections and (c) backscattering.

(b) omnidirectional propagation: $\mathrm{PhCs}$ feature an exclusive property that makes them capable of reflecting light propagating at any angle of incidence, i.e. there is no cut-off angle. The ray picture model clearly suggests that light propagating with normal or nearly-normal angle of incidence may form respectively a standing 
wave with no forward component, i.e. $k_{z}=0$ or a slow mode having very small forward components.

(c) Backscattering: Light propagating at parallel incidence (i.e. $k_{z}=0.5 \times 2 \pi / a$ ) experiences multiple reflections at each unit cell due to the periodic grating. The coherent interaction in phase and amplitude between the forward and backscattered propagating modes forms a standing wave with zero group velocity. When light propagates at nearly parallel incidence, i.e. within the vicinity of the Brillouin zone edge, the forward and backward travelling components still interact but slightly out of phase, giving rise to a slowly forward moving interference pattern: a slow mode. This may be pictured as a three steps forwardtwo steps backward motion as illustrated in Figure 3-2 (c). As one moves further away from the Brillouin zone the forward and backward travelling components are almost completely out of phase and total internal reflection becomes the dominant propagation mechanisms as in straight waveguides with regular group velocity. From such physical insight, one may think that slow light occurs only at the edge of the Brillouin zone in a reduced bandwidth. However, recent works on 2D $\mathrm{PhCs}$ with line defect, i.e. a 2D PhCs with a single line of missing hole, (W1 PhCs) [93-96] refuted this hypothesis showing that slow light may also be achieved inside the Brillouin zone over a wide bandwidth by increasing the coupling between modes of different orders naturally interacting with each other. These so-called engineered $\mathrm{PhCs}$ are of great interest for $\mathrm{EO}$ modulation applications owing to their artificially increased optical bandwidth. Interestingly, at the time of writing, this effect was not yet proposed nor demonstrated in LCWGs.

\subsubsection{Parity and TE, TM-like modes}

It should be pointed out that the dispersion relation shown above concentrates only on some particular guided modes of the periodic structure. Indeed, although pure TE/TM modes vanish in 3D, a TE/TM-like classification similar to that used in 2D problems, can be defined. However, because this terminology may sometimes be confusing, we can define the mode parity (even/odd) with respect to any plane of symmetry of the optical waveguiding structure and associate it to TE- or TM-like polarizations [97]. To be consistent with the PWE simulator conventions, we define TE-like (resp. TM-like) modes having no nodes in the 
center as being odd (resp. even) with respect the plane of symmetry of the structure $\mathrm{X}=0$ as illustrated in Figure 3-3. Alternatively and considering that the waveguide is surrounded by only one material (e.g. substrate BOX and covered by $\mathrm{SiO} 2$ have the same refractive index), we could also define another mirror plane $\mathrm{Y}=0$. However, this time the symmetry would be reversed and we would define TE-like (resp. TM-like) modes having no nodes in the center as being even (resp. odd) with respect the plane of symmetry of the structure $\mathrm{Y}=0$.

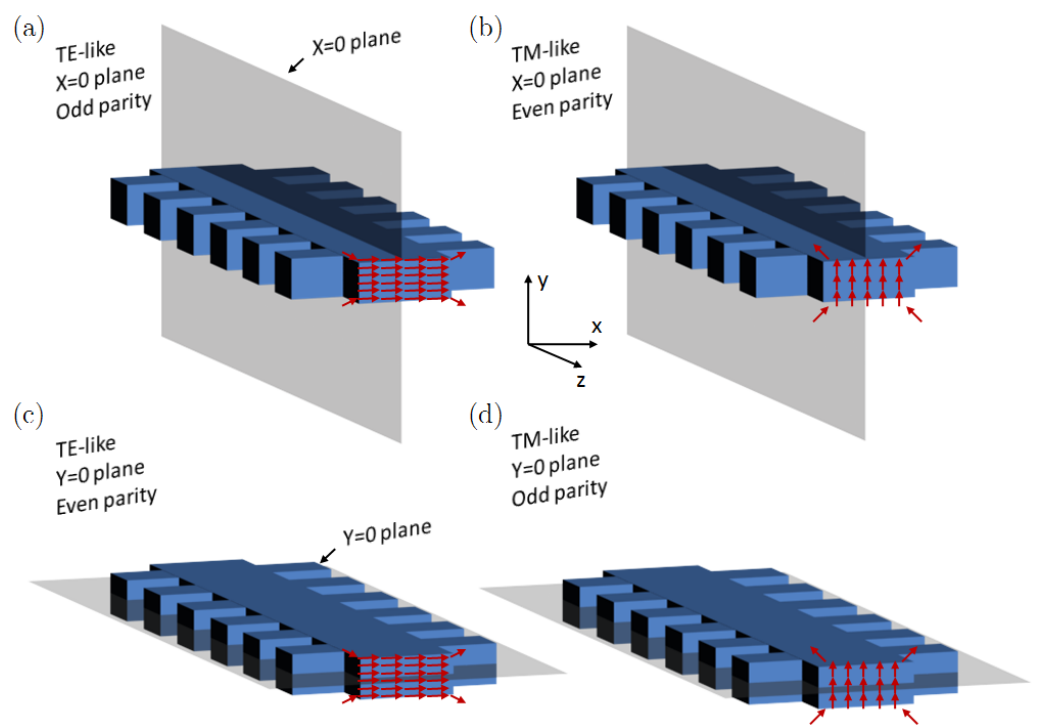

Figure 3-3: Electric field vectors predominantly (a) perpendicular to the mirror plane of the structure $(\mathrm{X}=0)$ and consistent with odd/TE-like modes (b) parallel to the mirror plane $(\mathrm{X}=0)$ and consistent with even/TM-like modes. (c) parallel to another mirror plane of the structure $(\mathrm{Y}=0)$ and consistent with even/TE-like modes (d) perpendicular another mirror plane of the structure $(\mathrm{Y}=0)$ and consistent with odd/TM-like modes

\subsubsection{Slow wave structure choice}

As previously seen, enabling light to propagate with slow group velocity in the form of Bloch modes is a matter of inducing multiple reflections inside any periodically patterned waveguide. Thereby, the wide variety of architectures potentially available should be only limited by the technological constraints, i.e. lithography resolution, etching process, and any other restrictive factors. The consequences of the fabrication process limitations and/or deviations when patterning slow wave structures may lead to several concerns such as periodicity breaking and shape variations over the propagation length which may eventually trigger disorder induced scattering loss as well some divergence compared to the expected optical behavior. However, the ceaseless progress in CMOS process 
optimization made over the years considerably reduced the gap initially existing between theoretical and real device performance. As a matter of fact, slow light has been successfully observed in passive silicon-on-insulator (SOI) dielectric structures such as PhCs [70, 91], coupled ring resonators [81, 98, 99] and more recently in LCWGs [100] as developed in the following. The choice of LCWGs as a passive approach to enhance the electro-optic modulation efficiency has been motivated by several factors listed hereafter:

(a) Geometry: Compared to other W1 PhCs which consists of closely packed elements, LCWGs features attractive geometrical properties as they consist only of a succession of simple narrow and wide rectangular dielectric elements. Nevertheless, when generating the mask layout, the designer should consider the specific lithography process limitations, essentially given by the wavelength of the illuminating radiation. Within the European framework ePIXfab [101], both standard 248 and $193 \mathrm{~nm}$ deep UV lithography processes are provided by CEALETI [102] and IMEC [103]. These processes are generally subject to optical proximity effects, which occur when the pattern of an illuminated structure interfere constructively of destructively with that of neighbouring elements, modifying therefore its size. For instance, in ultra dense W1 PhCs, the holes closer to the defect are smaller in size compared to the holes inside the bulk of the lattice. The same may happen when printing small sized and closely packed corrugated elements. To minimize such effects, the shape of the corrugations should be altered in advance on the layout if the minimum feature size is smaller than the wavelength of light used for the exposure. The designer should follow the design rules assessed by the two institutions and specify the minimum size on target or critical dimension (CD) to be reached by adjusting the exposure dose. Although also subject to optical proximity effects to a lesser extent, higher resolution at the expense of much longer exposure time is also achievable using electron-beam lithography, removing therefore the need of altering strongly the shape of the structure on the mask layout. This process is available at the Valencia Nanophotonics Technology Center (NTC)[104]. 
(a)

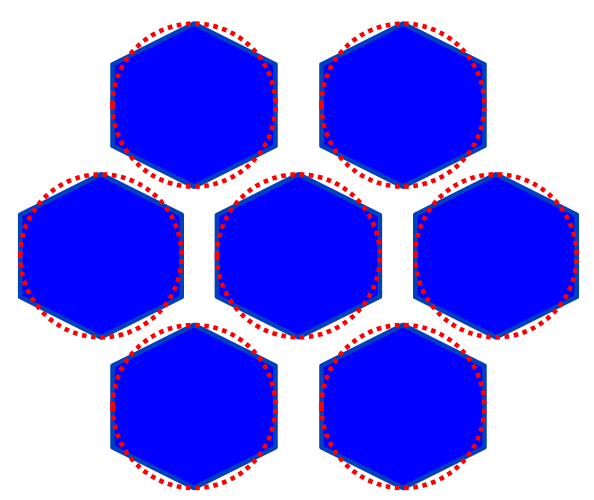

(b)

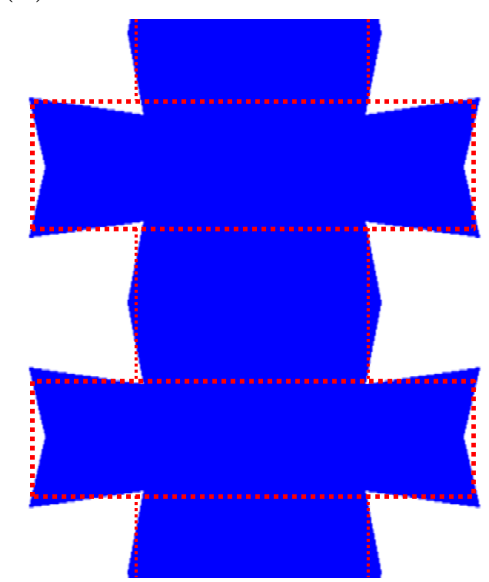

Figure 3-4: Examples of compensation for optical proximity effects in a (a) 2D triangular lattice $\mathrm{PhCs}$ and (b) 1D laterally corrugated waveguide. The blue structures stand for the design altered in advance and the red dashed line is the actual printed structure

(b) Losses: Among the variety of integrated slow wave structures (especially W1 $\mathrm{PhCs}$ ), the choice of LCWGs as a modulation enhancer has been made owing to its simpler geometry and expected lower sensitivity to fabrication disorder than W1 PhCs do. When operating below the light line, all excited modes, including Bloch modes are theoretically lossless in perfectly ordered W1 PhCs with high refractive index contrast. However, in real structures, radiative scattering may occur if on one hand the respective positions of holes with respect to each other is altered compared to the design (disorder) and/or on the other hand the holes exhibit some irregularities (sidewall roughness).

Firstly, due to the lack of confinement in the vertical direction, light tends to scatter in the vertical direction as shown in Figure 3-5. This radiative mechanism generates losses (per unit distance) that have been demonstrated to grow as the inverse of the group velocity $\left(1 / V_{g}\right)[105]$. Sometimes, scattered light may be recovered into the waveguiding region. This is because $\mathrm{W} 1 \mathrm{PhCs}$ reflect light back to the waveguide region even at large angles when operating inside the band gap.

Secondly, the most serious loss mechanism, which also originates from any imperfections such as positional disorder and/or sidewall roughness, is caused by back reflections that couple to the forward propagating modes in an uncontrolled and destructive manner. This effect is called backscattering and is thought to be responsible for losses (per unit distance) to scale with the square of the group velocity $\left(1 / V_{g}^{2}\right)[105]$. As a matter of fact, propagation losses in W1 PhCs operating in the slow light regime are still subject of controversy owing to the 
complexity of the mechanisms involved and the difficulties to separate them experimentally. The extrinsic nature of these issues have been subject to numerous publications[90, 106-108], questioning sometimes the suitability of W1 PhCs for low loss applications [105]. Another study [108] suggests that the backscattering loss mechanism only becomes dominant very near to the band edge, i.e. for very low group velocities (below c/100), whereas out-of-plane scattering dominates for moderately low group velocity (below c/20) scaling sublinearly with the latter rather than the expected inversely proportional dependence

Although there is no clear quantitative demonstration of what the main sources of losses in LCWGs are, one may point out the increased interaction between optical mode and waveguide defects such as sidewall roughness [109], period disorder (similar to hole position disorder in $\mathrm{W} 1 \mathrm{PhCs}$ ) as well as fast to slow mode coupling impedance mismatch. While the first two are fully related to the quality of the fabrication process, the third may be minimized by convenient tapering of the structure [110]. Besides, guided modes in LCWGs are fully index guided, whereas W1 PhCs feature both band gap guided and index guided modes. This means that no light recovery mechanism is possible in LCWGs and light scattered at any defect (disorder, roughness) will be able to escape freely within the low index cover cladding as for regular photonic wires.

In summary, both W1 PhCs and LCWGs are subject to optical propagation losses usually inherent to the fabrication deviations (roughness, disorder...) and specific waveguide geometry. In the fast light regime, state of the art devices were reported to feature propagation losses as low as $4.1 \pm 0.9 \mathrm{~dB} / \mathrm{cm}[111]$ and $0.92 \pm$ $0.14 \mathrm{~dB} / \mathrm{cm}$ [112] for W1 PhCs and 1D deep-etched LCWGs, respectively. Still, some doubts about the loss dependency against group velocity subsist when entering in the slow light regime. In W1 PhCs, backscattering losses, which grow as $1 / V_{g}^{2}$ are expected to be dominant over out-of-plane scattering losses, which grow as $1 / V_{g}$ (or sub-linearly[108]), but only for very low group velocity (i.e. below c/100). Interestingly, the need for such very low group velocity is not mandatorily required for modulation applications due the trade-off arising between useful optical bandwidth (i.e. the wavelength/frequency interval where the expected performance of the device is maintained), loss and low group velocity. Namely, the group velocity dependence upon frequency usually features a steep slope limiting the useful operating bandwidth. For lower group velocities, i.e. as we move closer to the band edge, this restriction becomes more severe, 
which clearly impairs potential applications. Therefore, we set our light speed objective to moderately low group velocity, i.e. around and beyond c/25. As we will see in this chapter, this range of group velocity values is sufficient to enhance drastically the modulation efficiency without increasing the insertion losses to unacceptable values on one hand, and without decreasing strongly the optical bandwidth on the other hand.

(a)

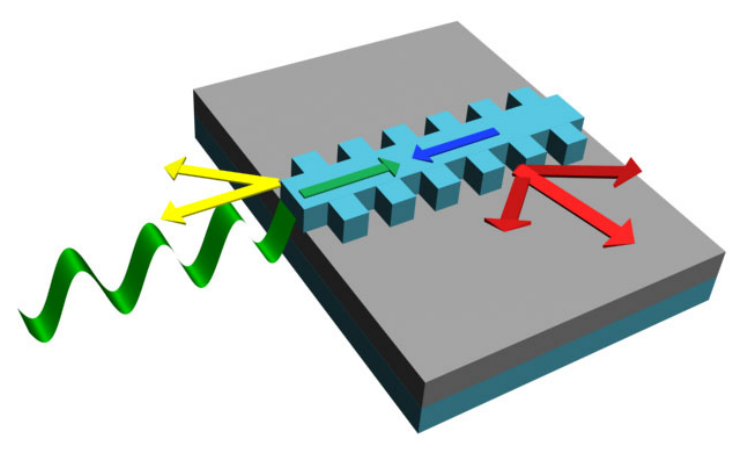

(b)

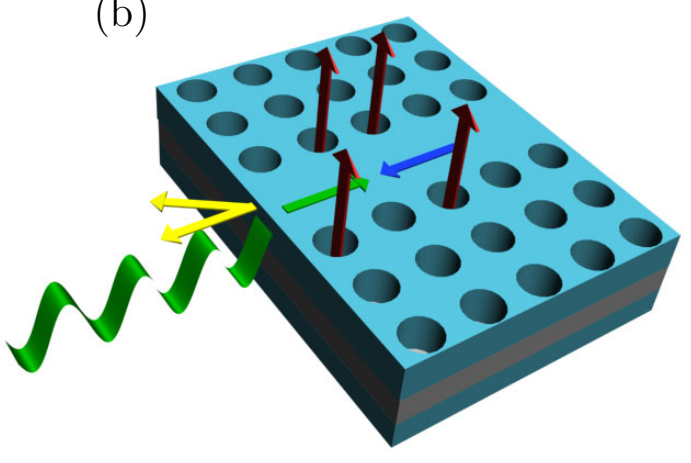

Figure 3-5: Schematic description of the different extrinsic loss mechanisms occurring (a) LCWGs (b) W1 PhCs. In (a) and (b), the green arrows illustrates the forward wave propagation motion. The yellow arrows depict the coupling losses due to fast to slow wave impedance mismatch. Note that this source of losses may be minimised by convenient tapering (not represented here) at both input and output of the waveguides $[110,113]$. The blue arrows depict the backscattered light due to waveguide defects (roughness, positional disorder) interfering destructively with the slow forward propagating wave. The red arrows describe in the scattering losses due to fabrication imperfections (roughness, disorder): in (a) light scatters freely in all directions into the cladding. (b) Because the allowed modes inside the line defect are confined by the photonic band gap in-plane light is has no choice but escaping in the vertical direction through the holes, i.e. where there is a lack of confinement.

(c) Compactness: In contrast with line defect W1 PhCs, LCWGs feature attractive aspects in terms of compactness since the effective refractive index difference effectively experienced by the optical mode can be high enough for a relatively small size ratio between the narrow and wide waveguide sections. Therefore, with the resolution constraints in mind, the designer should be able to generate corrugated waveguides, which maximum width would only be a few hundred nanometers wider than typical single mode silicon nanowires. This is of paramount importance for the case of modulators as the heavily doped regions 
(contacting regions) can be positioned closer to the waveguide, thus enhancing the intrinsic modulator speed.

By contrast, W1 PhCs require several repetitions of periodic elements (around ten) on both side of the defect to increase the confinement in-plane, therefore increasing the device footprint (Figure 3-6). In addition, since the metal contacts cannot be deposited on top of the holes for obvious reason, the contact to active area separation would become large thus probably affecting the intrinsic speed of the modulator unless good electrical design is made.

(a)

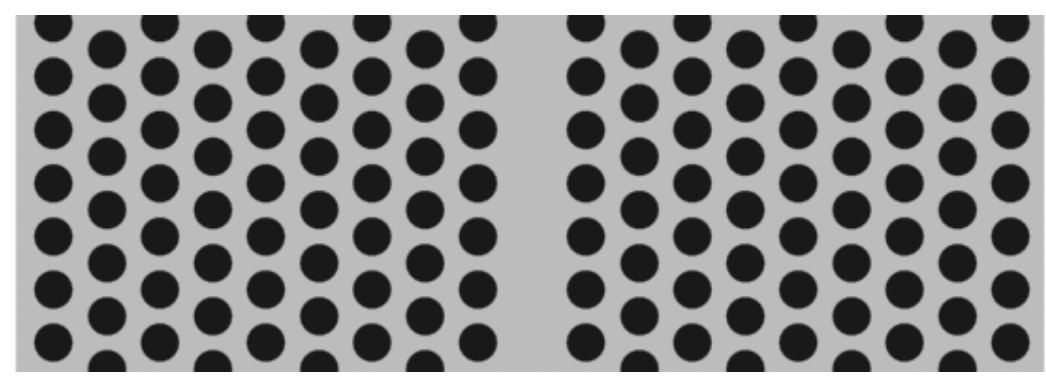

(b)

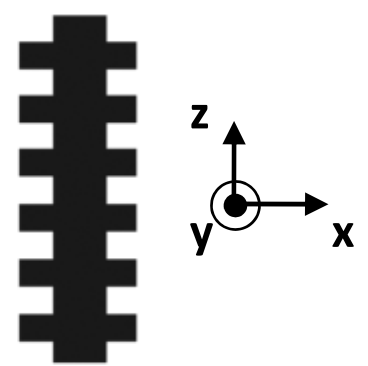

Figure 3-6: Footprint of a (a) W1 $\mathrm{PhC}$ with triangular lattice with 9 periods repeated on both side of the defect (b) LCWGs

(d) Computational time: Designing slow wave periodic waveguides in general may be made relatively simple owing to the modeling tools available. By contrast to the widely used FDTD method, the PWE method provides a fast a rigorous way to work out most of the properties of any periodic waveguide. If the FDTD algorithm may be used to describe the electromagnetic field evolution in most of the nanophotonic components, simulations may result time consuming for calculating the properties of periodic structures. On the contrary, the PWE algorithm is based on solving Maxwell's equations in the frequency domain leading to a rather simple eigenproblem. The solutions of the transformed time independent equations can be described as a superposition of plane waves, which forms the localized field distribution at any given frequency. The exactitude of the calculations is given by the number of plane waves involved in the calculations, which intrinsically set a trade-off between accuracy and computational time. Furthermore, in both FDTD and PWE calculations, the simulation region is to be defined by the user. The main difference between simulating periodic structures with FDTD or PWE is that the latter only requires the definition of a unit cell (or supercell in the case of more complex structures) that will be repeated in all simulation directions due to the periodic boundary 
conditions, while FDTD requires the inclusion of the whole waveguide within the simulation region. Therefore, since the computational time is closely related to the size of the simulation regions for both numerical methods, PWE outperforms FDTD in simulating periodic structures. With PWE, though, the supercell should be large enough to prevent identical neighboring cells to couple with each other. In other words, there should sufficient empty space between the boundaries of the supercell and those of the structure. Additionally, not all periodic structures requires the same computational time. The advantage of LCWGs over W1 PhCs is clearly that their unit cell may be much smaller resulting in a shorter simulation time as illustrated in Figure 3-7.

(a)

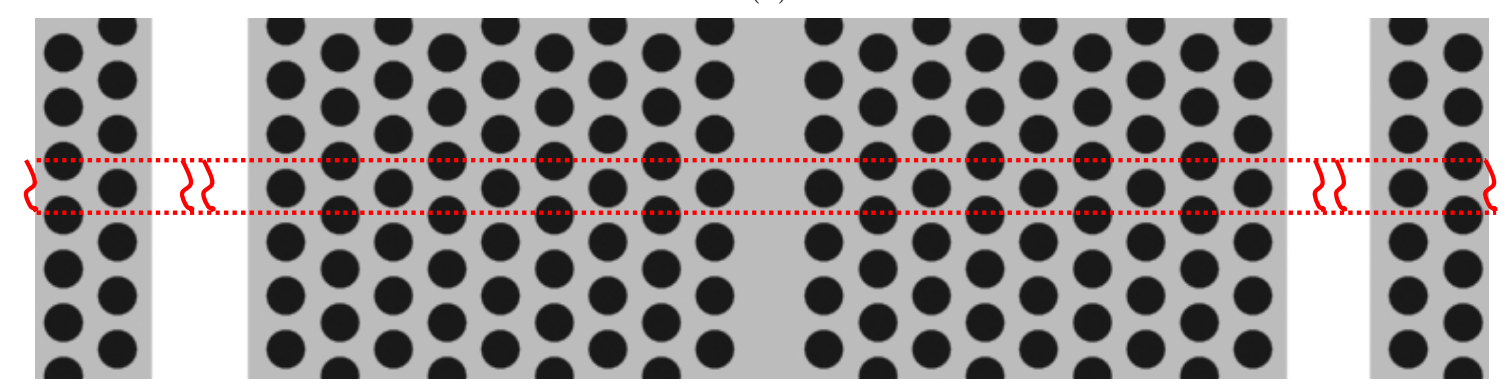

(b)
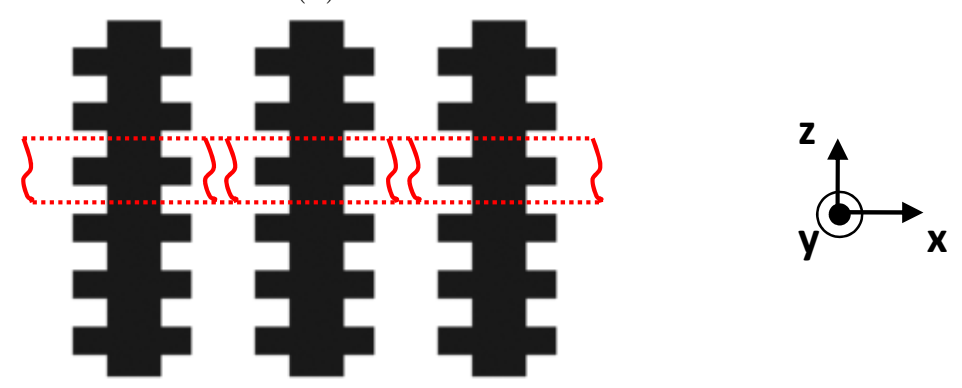

Figure 3-7: The red shaded line depicts the unit cell of a (a) W1 $\mathrm{PhC}$ with triangular lattice with 9 period repeated on both side of the defect (b) LCWGs. The unit cell is extended on both side of the periodic structure to avoid coupling with their periodically repeated neighbors. Although not depicted here, the same holds in the vertical direction (y-axis).

One of the main drawbacks of the PWE method is that it is unable to compute the losses within the structure whereas FDTD does. This is obviously of paramount importance especially in periodic structures in general where losses are still thought to limit their applicability. Nonetheless, experimental methods for propagation loss estimation in waveguides such as cut-back are expected to be more reliable as they include real fabrication process deviations. 
On the whole, the choice of a slow wave passive waveguide to enhance modulation is not straightforward as both W1 PhCs and LCWGs exhibit complementary and interesting features. However, the main characteristics that make LCWGs more attractive than their W1 PhC counterparts are: simpler geometry (less disorder issues), compactness, and less time consuming in terms of calculations. We believe that these arguments make LCWGs more suitable to demonstrate successfully high speed electro-optical modulation under an enhanced slow wave scheme.

\subsubsection{Slow wave structure overview}

As pointed out in the previous section, the slow wave waveguide has been designed by the PWE method. The slow wave behavior usually takes place close to the Brillouin zone edge in a rather narrow bandwidth. Just as for $\mathrm{W} 1 \mathrm{PhCs}$, in which the hole size and period essentially define the frequency where slow light propagation occurs, LCWG properties are entirely encompassed by a set of intrinsic parameters as illustrated in Figure 3-8 (a). Namely, the deep-etched version of this waveguide consists of an etched silicon epilayer of height $H$, forming an alternation of narrow and wide sections of respective widths $W$ and $W e$, repeated over a period $\Lambda$. The shallow-etched version of this waveguide is defined by the same set parameters except for the slab of height (h), which corresponds to the remaining silicon layer as shown in Figure 3-8 (b). The reason for proposing a shallow-etched version of the LCWG is because integrated silicon EO modulators require positioning the contacting regions on both side of the waveguide, i.e. where the cathode and anode may be deposited without overlapping the optical mode. These parameters have to be chosen in order to enable slow light to propagate around $1.55 \mu \mathrm{m}$ wavelength. The variety of parameters gives many degrees of liberty for designing a given LCWG. Therefore, to avoid time-consuming simulations, the range of parameters should be limited by setting some of them to a fixed value. In fact, the main dimensioning restrictions arise from the fabrication process capabilities. 
(a)

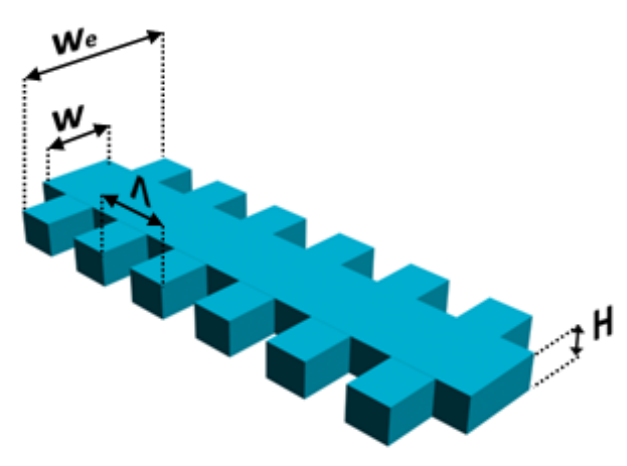

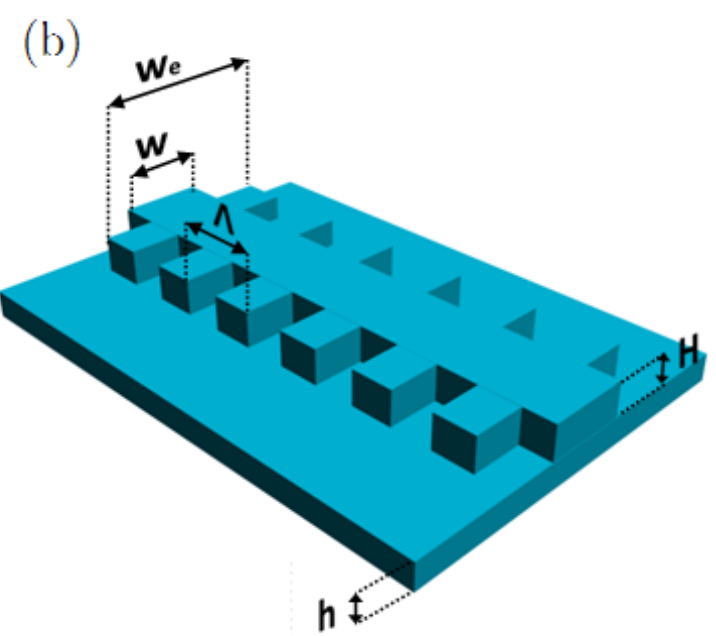

Figure 3-8: : Schematic of a (a) deep-etched LCWG defined by a set of 4 parameters: waveguide Height $(H)$, narrow and wide section widths, respectively $W$ and $W e$ and period $\Lambda$ and (b) shallow-etched LCWG with slab height $(h)$

\subsubsection{Parity and TE, TM-like modes in the shallow-etched slow light structure}

In contrast with the deep-etched version of the LCWG, the shallow-etched slow wave structure modal symmetry with respect to the $Y=0$ plane vanishes due to the presence of the slab. Hence, only modal symmetries with respect to the plane $\mathrm{X}=0$ are left over, as shown in Figure 3-9. In the following, the correspondence between modal parity and predominant polarization will be as follows. TE-like (resp. TM-like) modes with no nodes in the center correspond to odd (resp. even) parity with respect to the mirror plane $\mathrm{X}=0$.

(a)

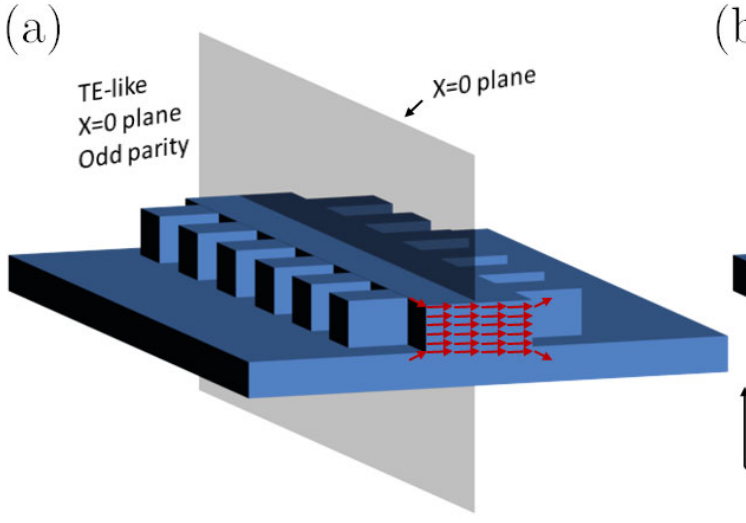

(b)

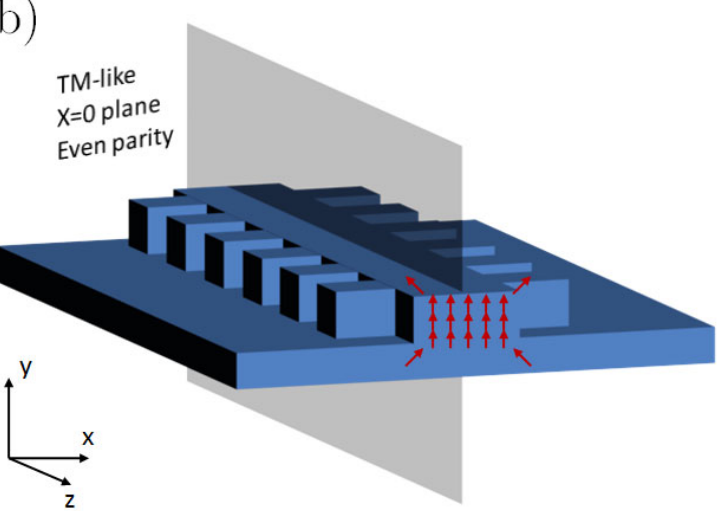

Figure 3-9: Electric field vectors predominantly (a) perpendicular to the mirror plane of the structure $(\mathrm{X}=0)$ and consistent with odd/TE-like modes (b) parallel to the mirror plane $(\mathrm{X}=0)$ and consistent with even/TM-like modes. 


\subsubsection{Fabrication constraints drive design}

Process limitations essentially reduce the flexibility on the choice of the structural parameters.

First, the choice of the silicon wafer conditions the total optical structure height ( $H$ or $H+h$ for the deep- and shallow-etched waveguide, respectively). For instance, The European wafer-scale fabrication service of silicon photonic integrated circuits (IC's) (ePIXfab [101]) commonly process wafer with 200 and $220 \mathrm{~nm}$ hick silicon epilayer on top of a $2 \mu \mathrm{m}$ thick Buried oxide Layer (BOX). Although other silicon thicknesses are available, these are more maturely processed and hence frequently used for cost-sharing purposes. In the following, we will consider the total height of the waveguide (including the slab) as $H+h=220 \mathrm{~nm}$.

Second, the slab height $(h)$ is mainly conditioned by two features, of which one is purely optical and the other electrical. On one hand, the single mode behavior of the waveguide should be ensured by decreasing as much as possible the slab height, but on the other hand, a fairly thick low resistive slab should be aimed at. Although the details of the electrical design are developed in the next section, the slab height thickness will lie in the range $h=50$ to $100 \mathrm{~nm}$.

Third, the last three parameters $W$ and $W e$ and $\Lambda$ are essentially limited by the resolution of the lithography process. Current state-of-the-art fabrication capabilities of photonic integrated circuits offer two different resist exposure processes. For both high-volume manufacturing and R\&D purposes, the $193 \mathrm{~nm}$ deep-UV lithography process coming from the microelectronics industry offers resolution down to approximately $120 \mathrm{~nm}$ (depending on the feature shape). Lower resolution at lower cost is also available through $248 \mathrm{~nm}$ deep-UV lithography. For pure research or low volume manufacturing, electron-beam lithography is available providing maximum a resolution of about $30 \mathrm{~nm}$. In any case, the minimum feature on the LCWGs should not be lower than $100 \mathrm{~nm}$. This sets a lower boundary on the minimum feature size of the slow wave waveguide.

Finally, the design of our LCWGs has been carried out with the aforementioned constraints, which are summarized in Table 3-2. 


\begin{tabular}{|c|c|}
\hline Total height $(\boldsymbol{H}+\boldsymbol{h})$ & $220 \mathrm{~nm}$ \\
\hline Slab height $(\boldsymbol{h})$ & $50-100 \mathrm{~nm}$ \\
\hline min. Feature size & $100-120 \mathrm{~nm}$ \\
\hline
\end{tabular}

Table 3-2: Summary of the waveguide dimensioning constraints

\subsubsection{Slow wave waveguide modeling}

The LCWGs have been modeled under BANDSOLVE ${ }^{\mathrm{TM}}$ from RSOFT $^{\mathrm{TM}}$ [74], which provides a 3D PWE simulation engine with user-friendly interface. The software solves Maxwell's equation in the frequency domain and computes the band diagram of any dielectric structure, which geometrical and optical parameters should be defined by the user. From the calculated band structure one may extract the majority of key parameters such as band gaps, group index, slow Bloch-mode field profile, effective index of refraction and any other parameters that may be of interest for the designer. Although the fabrication constraints reduce to some extent the degrees of liberty, the number of varying parameters remains high. The PWE method provides an accurate platform to work out the main optical properties of such a waveguide, however, one needs to carry out a fast preliminary study based on the beam propagation method (BPM) to avoid running too fast into a trial and error time-costly method. In the following, we describe the procedure used to model the LCWGs. Since the number of parameters of this particular structure is high $\left(W, W_{e}, W_{i} \Lambda\right.$, and $\left.h\right)$, we need at least to estimate the period at which the Bragg condition (given below) is satisfied in a particular structure. The following method aims at fixing both the period $\Lambda$, waveguide width and slab height in order to reduce the number of parameter to be varied from 5 to 2 . The Bragg condition is as follows.

$$
\Lambda n_{\text {eff }}=m \frac{\lambda_{\text {Bragg }}}{2}
$$

Where $\Lambda$ is the Bragg grating period, $n_{\text {eff }}$ the effective index of the optical mode, $m$ the mode order and $\lambda_{\text {Bragg }}$ the Bragg Wavelength. For the LCWG case, the formula needs to be slightly altered based on the following schematic. 


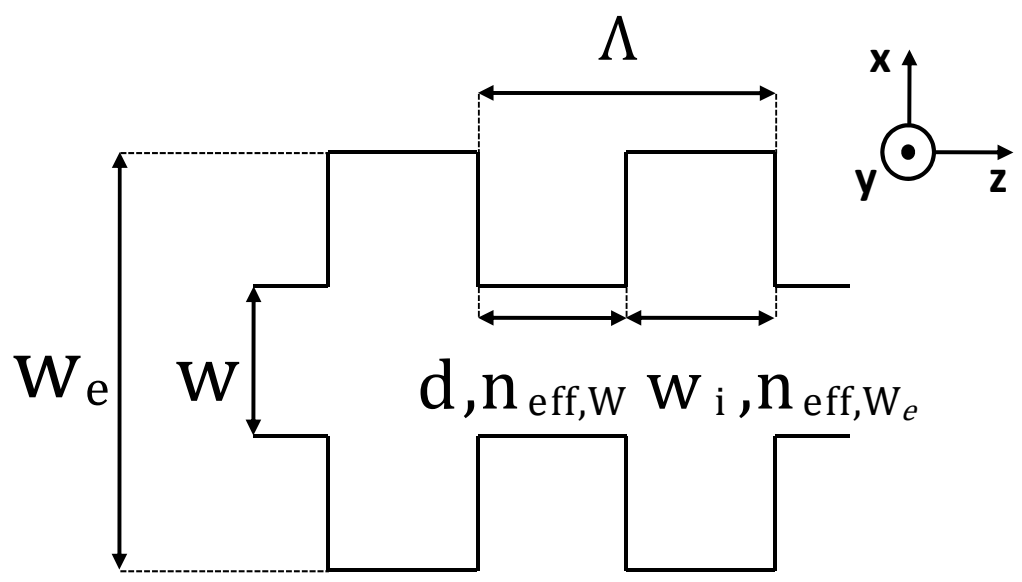

Figure 3-10: Top down view of the parameterized shallow-etched LCWG (the slab is not represented here)

$$
d n_{e f f, W}+W_{i} n_{e f f, W e}=m \frac{\lambda_{B r a g g}}{2}
$$

Considering a duty cycle $D=\frac{W_{i}}{\Lambda}=50 \%$, it follows:

$$
\frac{\Lambda}{2}\left(n_{\text {eff }, W}+n_{\text {eff, }, W_{e}}\right)=m \frac{\lambda_{\text {Bragg }}}{2}
$$

and

$$
\Lambda=m \frac{\lambda_{\text {Bragg }}}{\left(n_{\text {eff }, W}+n_{\text {eff }, W_{e}}\right)}
$$

Therefore by properly evaluating the effective indices of the modes propagating into the wide $\left(W_{e}\right)$ and narrow section $(W)$, one can derive the corrugated waveguide period $(\Lambda)$. In order to illustrate how this key parameter may approximately be estimated, we use a commercial full vectorial mode solver (FVMS) BEAMPROP ${ }^{\mathrm{TM}}$. The wide and thin section are set to arbitrary respective values of $W_{e}=550 \mathrm{~nm}$ and $W=300 \mathrm{~nm}$ and we assume a $h=50 \mathrm{~nm}$ thick slab. The mesh resolution is set in agreement with that which will be further used for finer but relatively time-saving PWE simulations. For the sake of completeness, the 3 dimensional mesh and waveguide parameters are indicated in Table 3-3. 


\begin{tabular}{|c|}
\hline Mesh Resolution ( $\boldsymbol{\mu m})$ \\
\hline $\mathrm{X}=0,015625$ \\
\hline $\mathrm{Y}=0,015625$ \\
$\mathrm{Z}=0,013010$ \\
\hline Refractive Index \\
\hline $\mathrm{nSi}=3,47641$ \\
\hline $\mathrm{nSiO}_{2}=1,44402$ \\
\hline
\end{tabular}

Table 3-3: Summary of the main FVMS simulation parameters

Figure 3-11 depicts 2D cut views of the fundamental modes propagating respectively through $300 \mathrm{~nm}$ and $550 \mathrm{~nm}$ wide rib silicon waveguides at $1.55 \mu \mathrm{m}$ wavelength. Then, from Equation (4.4) one can derive the period of a first order Bragg grating $\Lambda=332 \mathrm{~nm}$. This value provides a starting point for continuing the design with the PWE method.
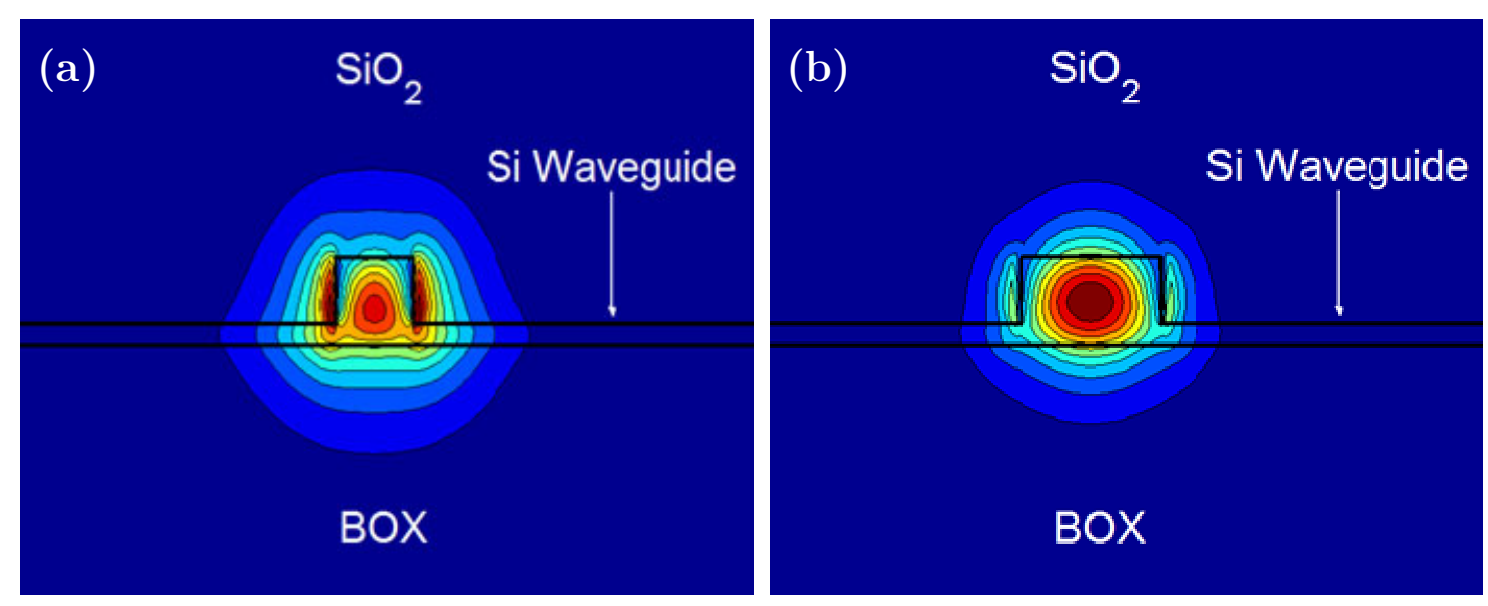

Figure 3-11: 2D cut of the fundamental mode profiles propagating through (a) $300 \mathrm{~nm}$ and (b) $550 \mathrm{~nm}$ wide waveguides @ $1.55 \mu \mathrm{m}$ wavelength. The effective index values are respectively (a) $n_{\text {eff, } w}=2.109451$ and $n_{\text {eff, } w e}=2.55557$

The remaining parameters are the width $\left(W_{e}\right)$ and length $\left(W_{i}\right)$ of the transversal corrugations. They are to be varied over a certain range, which boundaries are mainly delimited by the seeming fabrication constraints. For instance, the minimum separation $(\Lambda-W i)$ between two consecutive transversal elements should be $100 \mathrm{~nm}$ as well as the minimum length $W i$ should not be lower than $100 \mathrm{~nm}$ (Minimum resolution of deep UV lithography). In addition, the distance between the thin and wide waveguide edges should be as large as 
possible to minimize proximity effects (C.f. 3.3.3 (a)) while maintaining the waveguide operation around $1.55 \mu \mathrm{m}$ wavelength. In order to work out the Bragg wavelength $\lambda_{\text {Bragg }}$, i.e. the wavelength at which multiple back and forth reflections start taking place into the periodic waveguide, we run a $2 \mathrm{D}$ scan under a $3 \mathrm{D}$ PWE environment over the range $W_{i}=[100-200 \mathrm{~nm}]$ and $W_{e}=[500-800 \mathrm{~nm}]$. The Bragg wavelength corresponds to the photonic band edge operating point, that is, when the group velocity of the propagating wave theoretically vanishes.
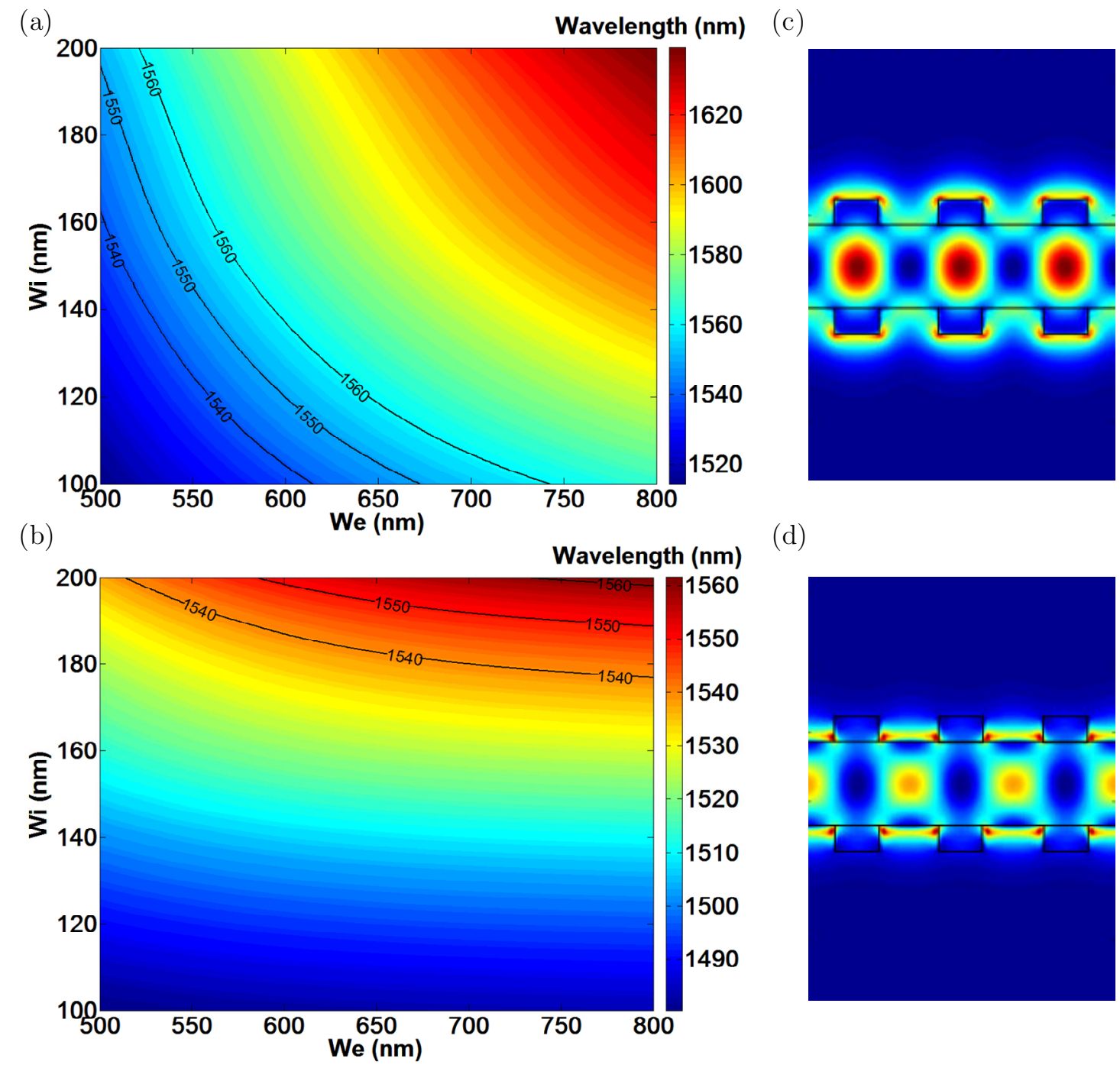

(d)

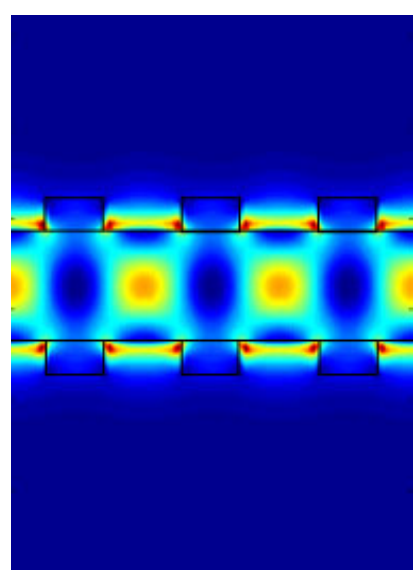

Figure 3-12: Contour plot of the Bragg wavelength @ the photonic band edge versus width $\left(W_{e}\right)$ and length $\left(W_{i}\right)$ of the transversal corrugations, respectively for the (a) fundamental and (b) first order mode. Corresponding top views of the square electric field of the (c) fundamental and (d) first order Bloch modes. $\Lambda=330 \mathrm{~nm}, W=300 \mathrm{~nm}$, $h=50 \mathrm{~nm}$ 
To make the waveguide parameter values compatible with the $5 \mathrm{~nm}$ grid imposed by the GDS file generation for deep-UV lithography, we conveniently round the period to $\Lambda=330 \mathrm{~nm}$. Although a slight blue shift (due to the shorter period) of the operating wavelength compared to BPM simulations is expected, a scan of the waveguide dimensions over the considered range should allow the compensation for such displacement. The PWE calculation results for the fundamental and first order modes, depicted in Figure 3-12, offer a set of parameter combinations $\left(W_{e}\right)$ and $\left(W_{i}\right)$ for which the waveguide exhibits slow light behavior at $1.55 \mu \mathrm{m}$ wavelength. Besides, a close look at the square electric field contours of both fundamental and first order gives an additional piece of information on the confinement of the Bloch modes into the waveguide.

The choice of the waveguide parameters relies mainly on the critical dimensions (CD), i.e. the minimum dimensions that may be resolved by the lithography process as already mentioned in section 3.3.3 (a). Since these LCWGs are expected to be fabricated with both $193 \mathrm{~nm}$ deep-UV and electron beam lithography process, 4 early designs consisting of different combinations of $\left(W_{e}\right)$ and $\left(W_{i}\right)$ were selected for passive fabrication runs with CD of about $100 \mathrm{~nm}$ in order to tackle potential fabrications issues. The initial design dimensions are summarized below (Table 3-4).

\begin{tabular}{|c|c|c|c|c|c|c|c|}
\hline $\begin{array}{c}\text { Design } \\
\boldsymbol{n}^{\boldsymbol{o}}\end{array}$ & $\begin{array}{c}\boldsymbol{W} \\
(\mathbf{n m})\end{array}$ & $\begin{array}{c}W \boldsymbol{i} \\
(\mathbf{n m})\end{array}$ & $\begin{array}{c}W e \\
(\mathbf{n m})\end{array}$ & $\begin{array}{c}\Lambda \\
(\mathbf{n m})\end{array}$ & $\begin{array}{c}\boldsymbol{\Lambda}-\boldsymbol{W} \boldsymbol{i} \\
(\mathbf{n m})\end{array}$ & $\begin{array}{c}\text { slab height } \\
(\mathbf{n m})\end{array}$ & $\begin{array}{c}\text { mode } \\
\text { order }\end{array}$ \\
\hline 1 & 300 & 100 & 650 & 330 & 230 & 50 & 0 \\
\hline 2 & 300 & 125 & 600 & 330 & 205 & 50 & 0 \\
\hline 3 & 300 & 150 & 550 & 330 & 180 & 50 & 0 \\
\hline 4 & 300 & 170 & 530 & 330 & 160 & 50 & 0 \\
\hline 5 & 400 & 140 & 800 & 320 & 180 & 50 & 1 \\
\hline 6 & 300 & 150 & 550 & 330 & 180 & 50 & 0 \\
\hline 7 & 300 & 150 & 800 & 310 & 160 & 50 & 0 \\
\hline
\end{tabular}

Table 3-4: Summary of the shallow-etched LCWGs parameters $(\mathrm{h}=50 \mathrm{~nm})$

As can be seen, the waveguide width $(W)$, period $(\Lambda)$ wide section width $\left(W_{e}\right)$ were altered to increase the variety of designs. For instance, the waveguide width $(W)$ and wide section width $\left(W_{e}\right)$ of design $n^{\circ} 5$ were increased to simultaneously 
reduce the waveguide losses, and make it more accessible to the existing standard lithography techniques. As a result, the effective index increases and the Bragg wavelength increases accordingly. This in turn imposes to reduce the Period $(\Lambda)$ in order to compensate for such effect and to position the Bragg wavelength (or photonic band edge) back to around $1.55 \mu \mathrm{m}$ wavelength. To limit the period reduction and hence the distance between two consecutive transversal elements $(\Lambda-W i)$, the Bragg wavelength may be chosen to match the first order mode $(\mathrm{m}=1)$.

For electro-optic modulation applications, the slab height is closely related to the modulation efficiency and speed. Concretely, a thick slab helps charge carriers to experience lower electrical resistance as will be discussed in the following section. Therefore, it is relevant to assess the performance of shallowetched LCWGs while dealing with tight design constraints. Following the same method as that abovementioned, designs featuring a $100 \mathrm{~nm}$ thick slab were worked out. These 3 designs, extracted from Figure 3-13 (a), (b) and (c) are respectively summarized hereafter.

\begin{tabular}{|c|c|c|c|c|c|c|c|}
\hline $\begin{array}{c}\text { Design } \\
\boldsymbol{n}^{\boldsymbol{o}}\end{array}$ & $\begin{array}{c}\boldsymbol{W} \\
(\mathbf{n m})\end{array}$ & $\begin{array}{c}\boldsymbol{W i} \\
(\mathbf{n m})\end{array}$ & $\begin{array}{c}W \boldsymbol{e} \\
(\mathbf{n m})\end{array}$ & $\begin{array}{c}\boldsymbol{\Lambda} \\
(\mathbf{n m})\end{array}$ & $\begin{array}{c}\Lambda-W \boldsymbol{i} \\
(\mathbf{n m})\end{array}$ & $\begin{array}{c}\text { slab height } \\
(\mathbf{n m})\end{array}$ & $\begin{array}{c}\text { mode } \\
\text { order }\end{array}$ \\
\hline 1 & 400 & 160 & 700 & 305 & 145 & 100 & 1 \\
\hline 2 & 300 & 170 & 600 & 300 & 130 & 100 & 0 \\
\hline 3 & 200 & 150 & 700 & 305 & 160 & 100 & 0 \\
\hline
\end{tabular}

Table 3-5: Summary of the shallow-etched LCWGs parameters $(\mathrm{h}=100 \mathrm{~nm})$ 
(a)
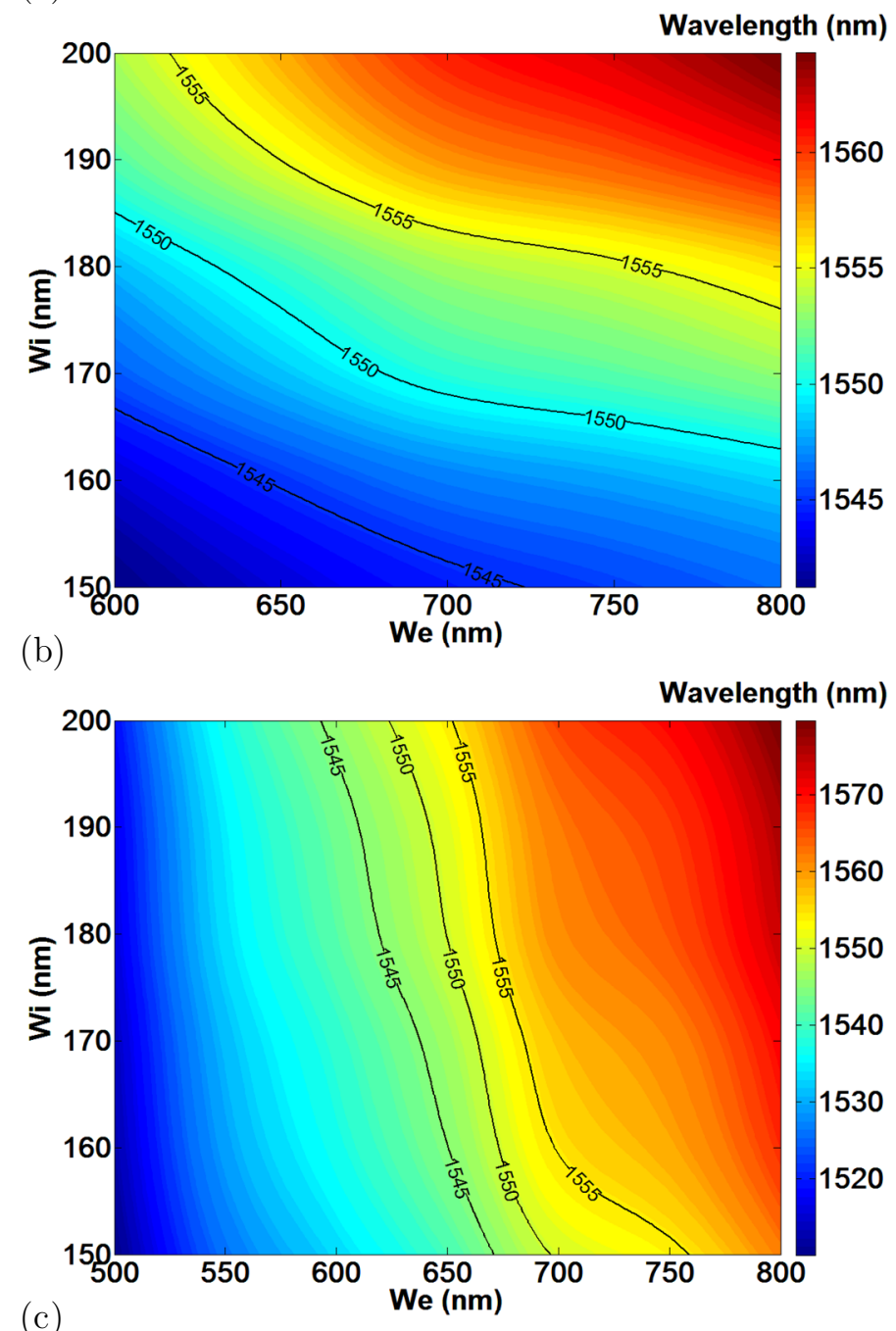

(c)

Wavelength $(\mathrm{nm})$

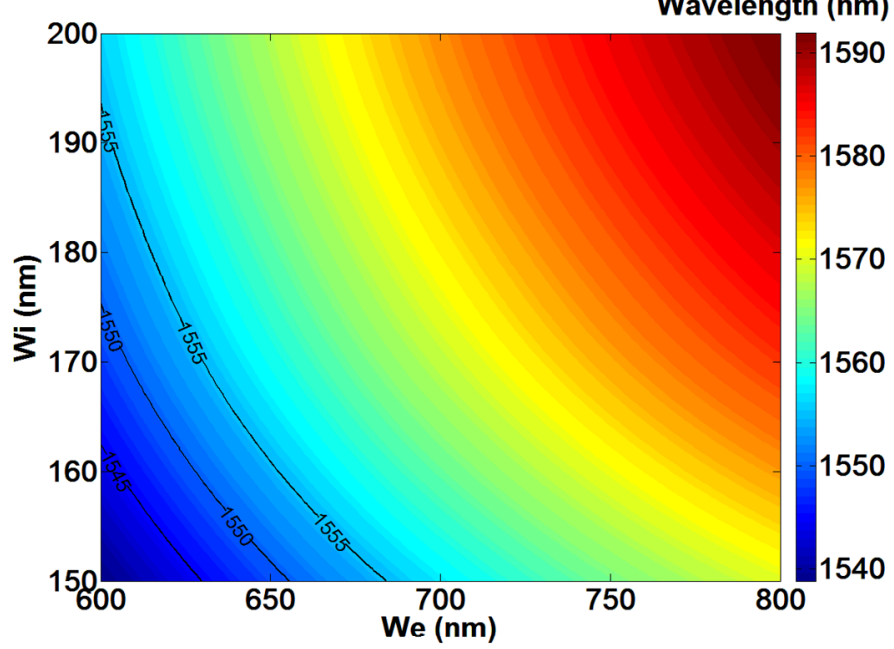

Figure 3-13: Contour plot of the Bragg wavelength @ the photonic band edge versus width $\left(W_{e}\right)$ and length $\left(W_{i}\right)$ of the transversal corrugations, respectively for the (a) first order mode. $(\Lambda=305 \mathrm{~nm}, W=400 \mathrm{~nm})(\mathrm{b})$ fundamental mode $(\Lambda=300 \mathrm{~nm}, W=300$ $\mathrm{nm}$ ) and (c) fundamental mode $(\Lambda=305 \mathrm{~nm}, W=200 \mathrm{~nm})$. 
Overall, our shallow-etched LCWG design method has been presented as a combination of two different simulation engines: PWE and BPM. The high refractive index contrast inherent to SOI technology causes the grating period $\Lambda$ to be almost twice smaller $(300-330 \mathrm{~nm})$ than in Bragg grating fibers where the optical mode confinement is lower. Consequently, to meet standard lithography capabilities, some restrictions on the corrugated elements size arise. For standard $193 \mathrm{~nm}$ deep-UV lithography, proximity effects are expected to causes slight geometrical differences between the design and the actual fabrication. Such deviations are expected to be much lower using high resolution e-beam lithography. Finally, a variety of shallow-etched LCWGs designs has been worked out for both $\mathrm{h}=50 \mathrm{~nm}$ and $\mathrm{h}=100 \mathrm{~nm}$ thick slab versions, paving the way for further fabrication and optical characterization.

\subsection{Electrical Design}

\subsubsection{Slow wave phase shifter description}

Whilst designing passive silicon slow wave structures is a fundamental step, practical active devices require further work. Here, we aim at showing that slow wave structures are poised to enhance strongly the performance of active components by carrying out an analysis of electro-optic modulation (SW EOM) in slow wave LCWGs. In what follows, we provide a description of such a device.

The passive optical structure consists of a $W=300 \mathrm{~nm} \times H=220 \mathrm{~nm}$ single mode silicon rib waveguide with a $h=50 \mathrm{~nm}$ thick slab and corrugated elements of dimensions $W_{e}$ and $W_{i}$ repeated over a period $\Lambda=330 \mathrm{~nm}$. As discussed in the previous section, this periodic repetition of identical transversal elements gives rise to slow light propagation at the edge of the Brillouin zone (photonic band edge). Thus, light propagates with lower group velocity over a certain frequency range (around the Bragg wavelength), depending upon the width $\left(W_{e}\right)$ and length $\left(W_{i}\right)$ of the transversal elements. The electrical structure, an asymmetrical $p n$ junction shown Figure 3-14, was proposed and demonstrated in references [35, 114 ] in which it turned out to be an efficient modulation scheme. Consequently, this particular structure has been employed in the electrical simulations in association with LCWGs to show the improvement they may bring with respect to conventional rib waveguides. 


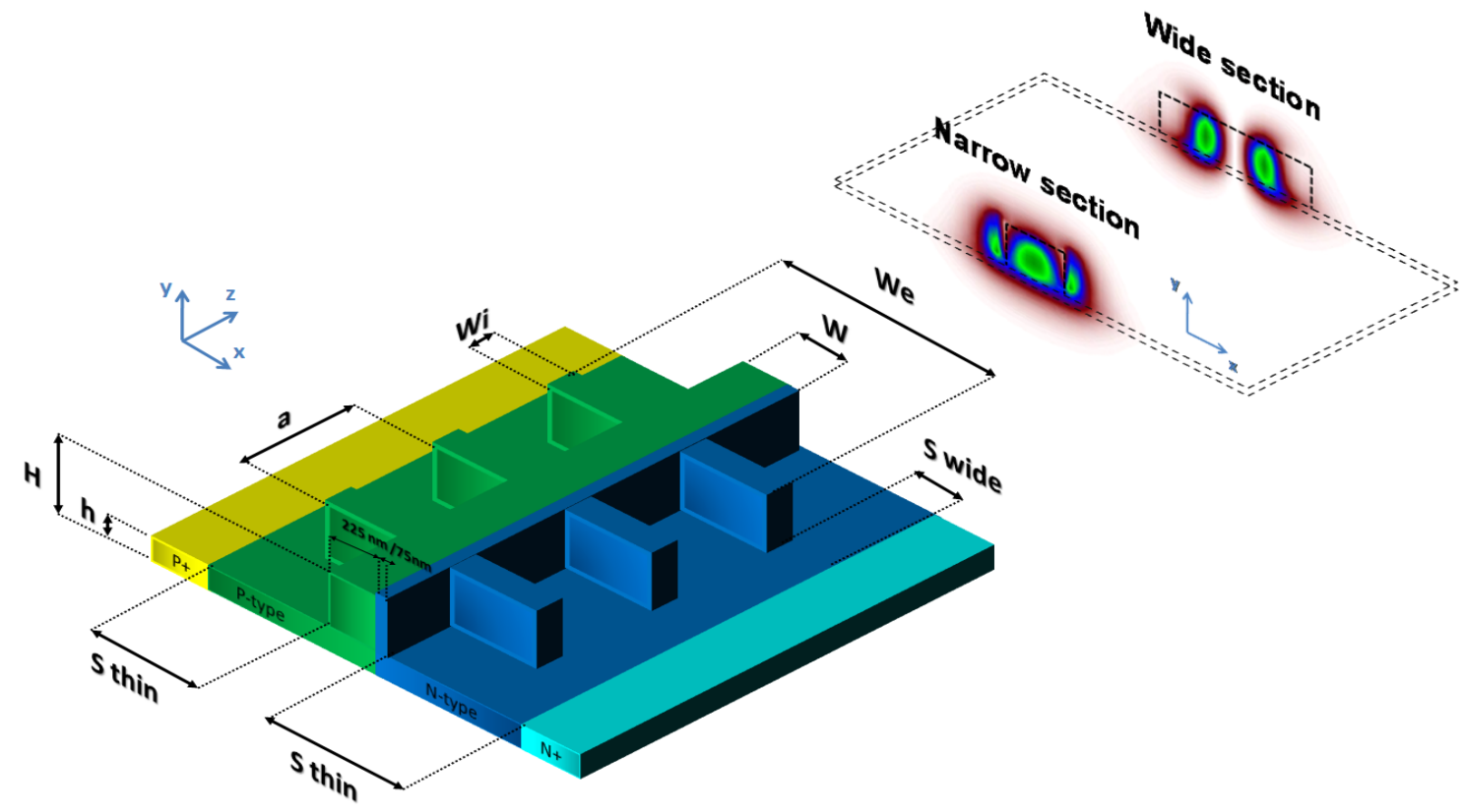

Figure 3-14: Schematic of the slow wave phase shifter (SWPS) and fundamental slow mode $\left|E_{x}\right|^{2}$ profile respectively in the narrow and wide section.

The SWPS is divided into two $n$-type and $p$-type doped regions, which respective net doping concentrations are $6.10^{17} \mathrm{~cm}^{-3}$ and $2.10^{17} \mathrm{~cm}^{-3}$, respectively. Both $\mathrm{n}^{+}$an $\mathrm{p}^{+}$doped regions are implanted with a concentration of $1.10^{20} \mathrm{~cm}^{-3}$ to ensure good ohmic contacts and are positioned at a distance $S_{\text {wide }}=1 \mu \mathrm{m}$ away from the thin section of the waveguide. The parameter $S_{\text {thin }}$ is to be determined depending on the dimensions of both $W_{e}$ and $W_{i}$. The passive device design guideline may be orientated by the doping concentrations into the waveguide as the optical properties of the corrugated structure are slightly modified by the presence of impurities. Therefore, the doping concentration distributions along the waveguide cross section, though only slightly altering the refractive index distribution with respect to a non doped waveguide have even so been taken into account in the calculations. The refractive index distribution for a doped waveguide is derived from Soref's expression [16] at $\lambda=1.55 \mu \mathrm{m}$ with no bias applied:

$$
n=n_{e}+n_{h}+n S i=-\left(8.8 \cdot 10^{-22} N_{e}+8 \cdot 5 \cdot 10^{-18} N_{h}^{0.8}\right)+n S i
$$

The density of electrons $N_{e}$ and holes $N_{h} N_{h}$ are calculated solving Poisson's equation under the semiconductor CAD environment provided by ATLAS [115]. 
The refractive index of undoped silicon is $n S i=3.47641$ The determination of $W_{e}$, and $W_{i}$ has been made as a result of PWE calculations, which include the altered refractive index distribution calculated above in Equation (4.5). The two dimensional scan was carried out in order for wavelengths surrounding $1550 \mathrm{~nm}$ to match the edge of the Brillouin zone, i.e. the slow wave region. Figure 3-15 below illustrates the results of such calculations.

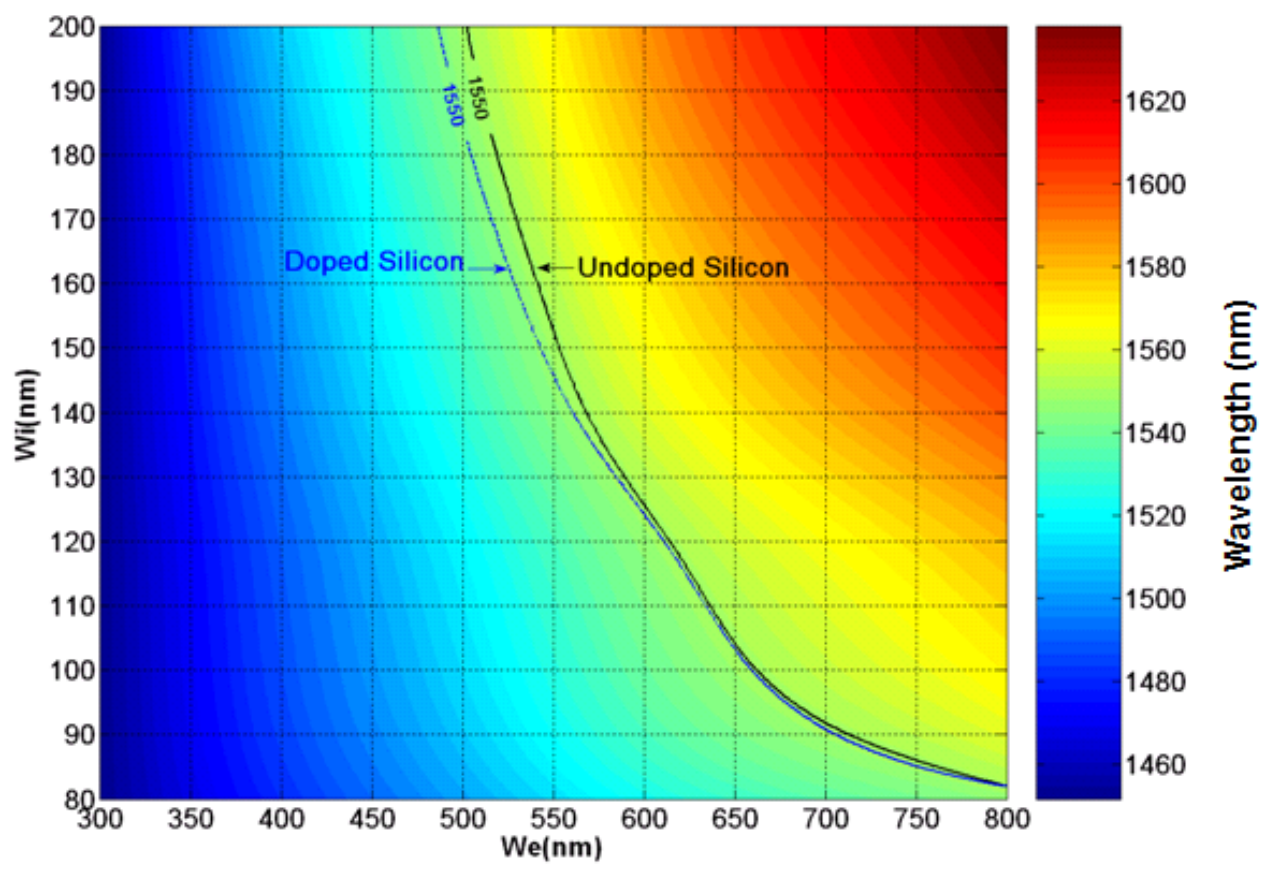

Figure 3-15: Contour plot of the fundamental mode wavelengths in the slow light regime versus varying periodic elements size for undoped silicon corrugated waveguides. The blue contour line has been derived including the slight refractive index variation at $\lambda=1.55 \mu \mathrm{m}$ due to the impurities introduced into the waveguide. Results are plotted at the band edge $(k=0.5 * 2 \pi / \mathrm{a})$.

From Figure 3-15, the basic couple of parameters $\left(W_{i}, W_{e}\right)$ can be extracted to set the corrugation dimensions. The multiple choices offered are oriented by two primary fabrication concerns:

1. The minimum separation $\left(\Lambda-W_{i}\right)$ between two consecutive transversal elements should be $100 \mathrm{~nm}$ as well as the minimum length $W_{i}$ should not be lower than $100 \mathrm{~nm}$ to avoid proximity effect

2. The corrugations should not be closer than $500 \mathrm{~nm}$ from the $p+$ and $n+$ highly doped regions, i.e. $S_{\text {wide }}>500 \mathrm{~nm}$ to avoid excessive optical losses. 
Among many other suitable dimensions, those selected for our enhanced modulator to work at $1.55 \mu \mathrm{m}$ wavelength in the slow light regime are set to $W_{e}=650 \mathrm{~nm}$ and $W_{i}=100 \mathrm{~nm}$, although further device optimizations and experimental tests could adjust slightly these values. The following section shows how the slow wave waveguides enhance the modulator performances in terms of refractive index change and interaction length.

\subsubsection{DC analysis}

The SWPS performance is first evaluated under static regime for varying applied drive voltages, i.e. when the modulation is time-independent. As discussed previously, enhancing the interaction relies on the intensity of the interaction between the free carriers and the slow optical mode propagating through a LCWG. Intuitively, the slower the mode propagates, the greater the interaction with free carriers. Hence, the interaction length can be strongly reduced compared to conventional rib waveguide-based EO modulators, resulting in a shorter phase shifter and/or lower power requirements.

The SWPS is operated under reverse bias from $V_{\text {ini }}=0 \mathrm{~V}$ up to $V_{\text {fin }}=-10 \mathrm{~V}$. As a result, the space charge region width increases as carriers are depleted from the junction. The effect of the applied drive voltages on the real refractive index distribution is calculated in $2 \mathrm{D}$ using again Soref's expression at $\lambda=1.55 \mu \mathrm{m}$, shown in Equation (4.6).

$$
\begin{aligned}
n(V) & =n_{e}(V)+n_{h}(V)+n S i(V) \\
& =-\left(8.8 .10^{-22} N_{e}(V)+8.5 .10^{-18} N_{h}(V)^{0.8}\right)+n S i
\end{aligned}
$$

The real refractive index change $\Delta n=n\left(V_{\text {fin }}\right)-n\left(V_{\text {ini }}\right)$ distributions in both thin and wide waveguide sections have been worked out for different drive voltages as illustrated in Figure 3-17. These real refractive index distributions have been then included into a PWE simulator by means of an in-house conversion program (Appendix A) in order to work out the effective index change as a function of the applied reverse bias variations. The effective index change results from the overlap between the optical mode and the real refractive index distribution. The design method flow for the DC analysis of the slow wave modulator is summarized in Figure 3-16. 

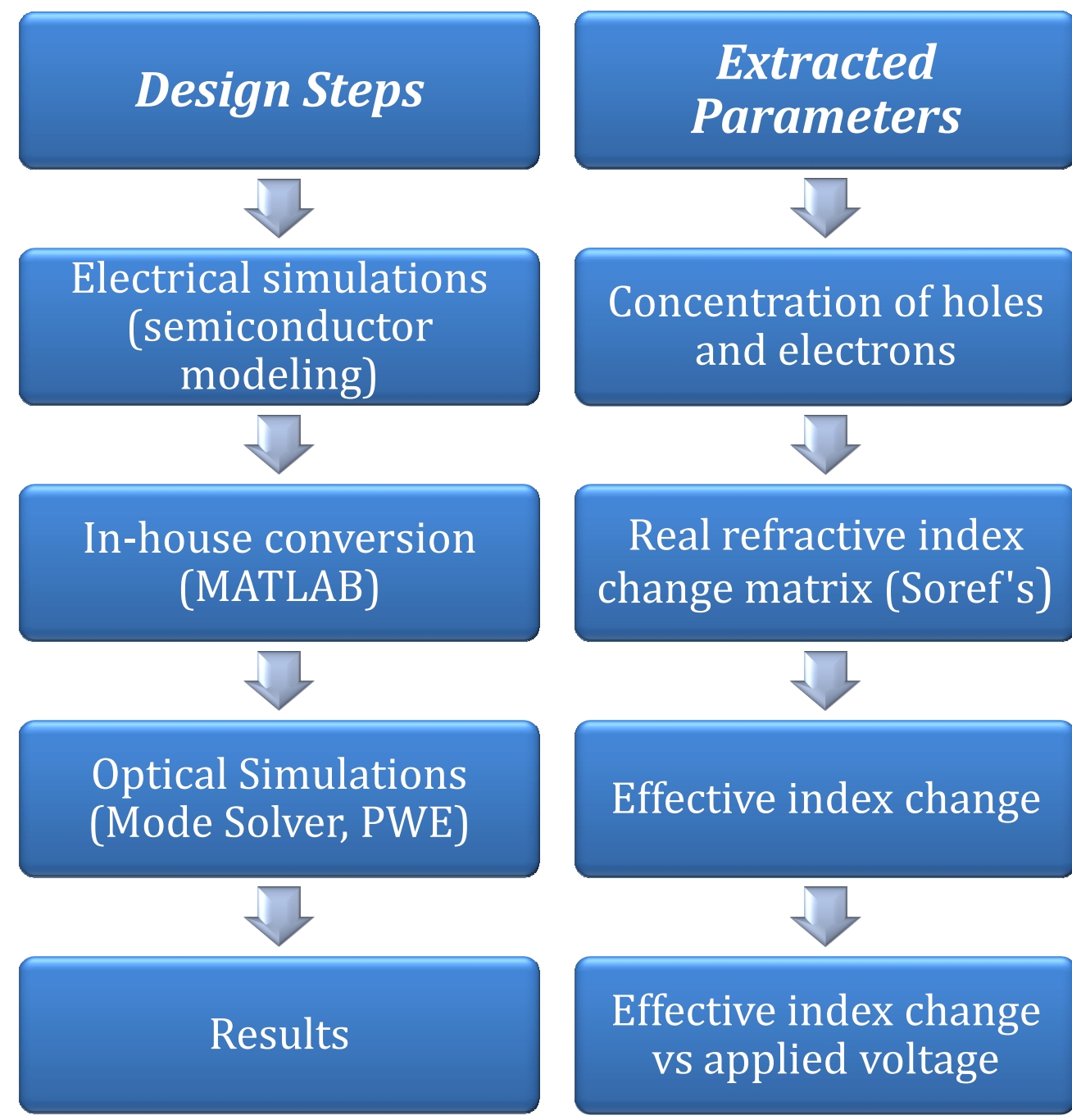

Figure 3-16: Design method flow for the DC analysis of the slow wave modulator 


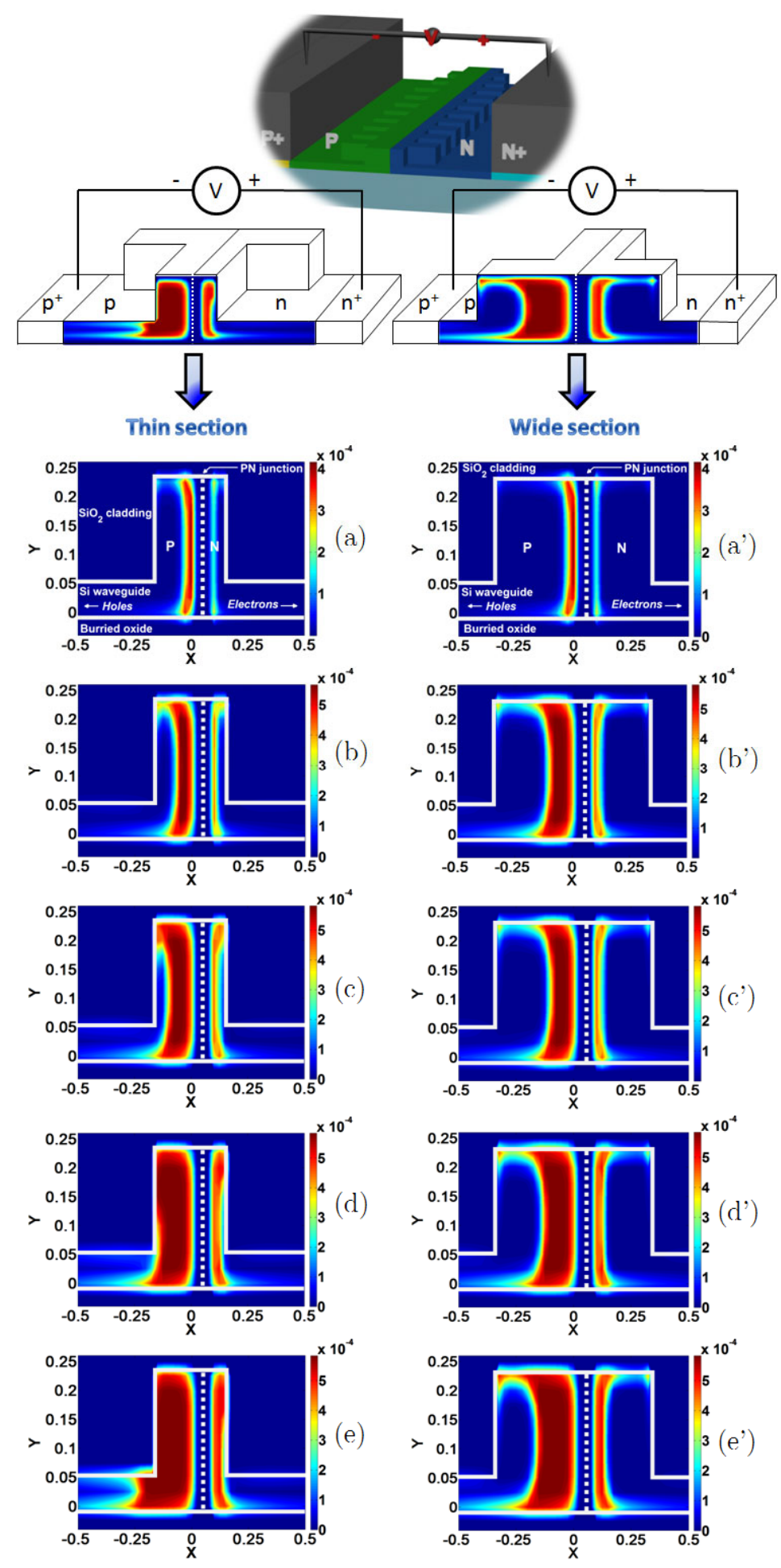

Figure 3-17: real refractive index change $\Delta n$ under different applied drive voltages (a), (a') $\Delta V=-2 \mathrm{~V} ;$ (b), (b') $\Delta V=-4 \mathrm{~V} ;$ (c), (c') $\Delta V=-6 \mathrm{~V} ;$ (d), (d') $\Delta V=-8 \mathrm{~V} ;$ (c), (c') $\Delta V=-$ $10 \mathrm{~V}$ for the thin and wide sections of the LCWG, respectively. The junction is in steady state. Waveguide cross section boundaries are represented by the white plain lines

Figure 3-18 shows the effective index change versus applied reverse bias for varying group index $\left(n_{g}\right)$ as a result of our computational method. These results 
clearly suggest that the effective index change is strongly increased as the group index does. To provide a clear picture of the improvement our slow wave modulator might offer, the effective index change enhancement has been quantified by calculating the ratio between the effective index change achieved in a LCWG as well as in a conventional rib waveguide (embedded in the same electrical structure) of dimensions $300 \mathrm{~nm}$ x $220 \mathrm{~nm}$ and with a $50 \mathrm{~nm}$-thick slab.

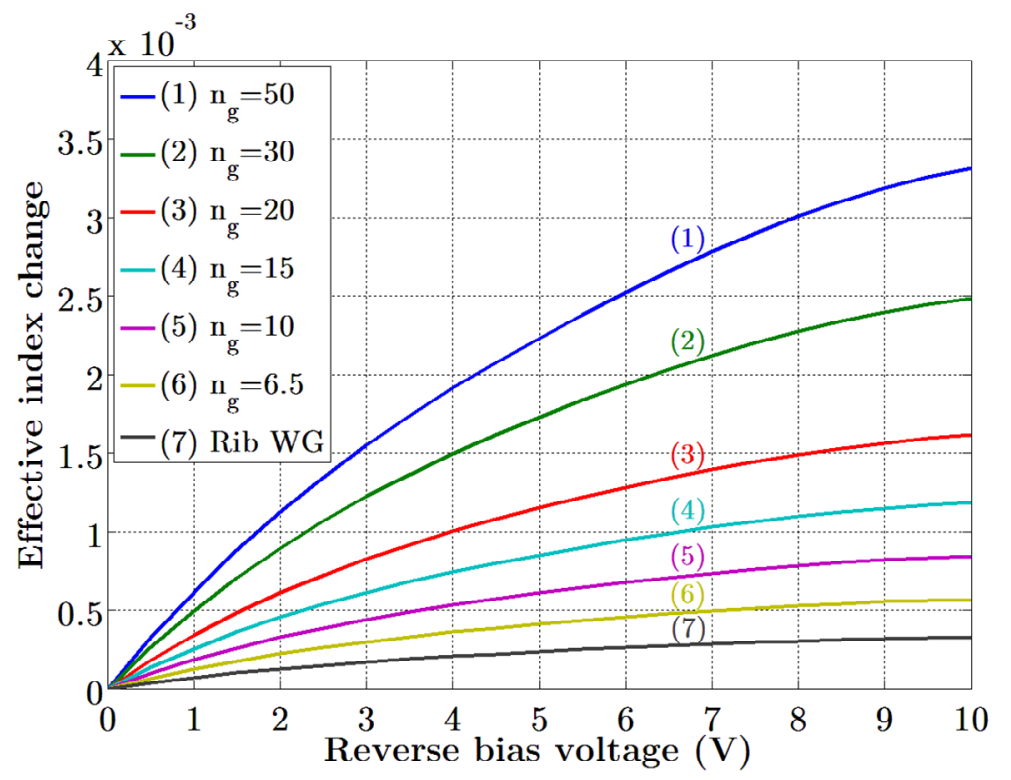

Figure 3-18: DC response of the slow wave phase shifter: Effective index change versus applied reverse bias for varying group index

Such a parameter is denoted the enhancement factor (EF) and is defined as follows:

$$
E F=\Delta n e f f_{c o r r} / \Delta n e f f_{r i b}=\Delta \varphi_{\text {corr }} / \Delta \varphi_{\text {rib }}
$$

Where $\Delta n e f f_{\text {corr }}\left(\right.$ resp. $\left.\Delta \varphi_{\text {corr }}\right)$ and $\Delta n e f f_{r i b}$ (resp. $\Delta \varphi_{\text {rib }}$ ) are the change in effective index (resp. phase shift) in the LCWG and rib WG, respectively. This EF has been calculated as a function of the applied voltage as illustrated in Figure 3-19. 


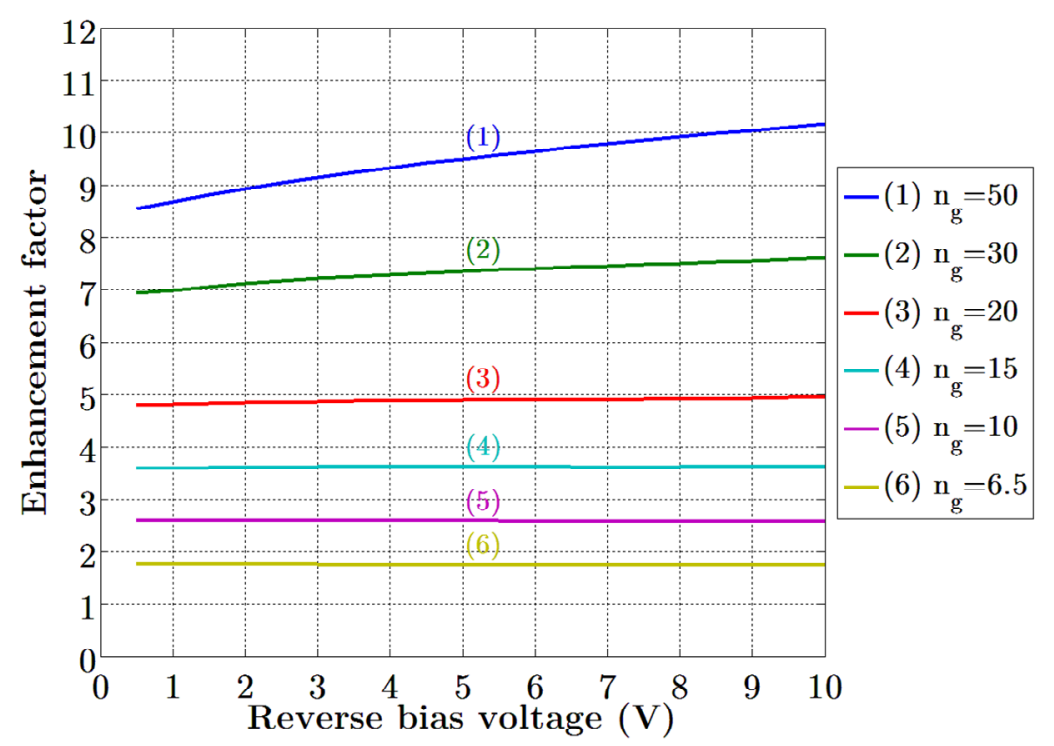

Figure 3-19: Figure of merit versus applied reverse bias calculated from Equation (4.7) for varying group index.

The first observation that can be made is the strong effective index variation enhancement achieved in the slow light regime compared to that achieved the conventional rib waveguide. The information that can be directly extracted from Figure 3-19 is the size reduction with respect to rib waveguide EOM. As an example, the refractive change $\Delta n_{e f f}=2.616 .10^{-4}$ or an applied drive voltage variation of $-6 \mathrm{~V}$ in a rib waveguide, leads to an interaction length $L_{\pi, r i b}=$ $\lambda / 2 \Delta n_{\text {eff }}=2962.5 \mu \mathrm{m}$ to achieve a $\pi$-phase shift. Figure $3-19$ shows that this refractive index change is almost one order of magnitude greater ( $\approx 9.65)$ for a group index $n_{g}=50$ at $-6 \mathrm{~V}$. The resulting length to achieve the same phase shift in the slow wave device is then only $L_{\pi, c o r r}=307 \mu \mathrm{m}$. For the sake of completeness, the interaction length versus applied drive voltage and for varying group index is depicted in Figure 3-20. These results suggest that even for relatively low group indices $\left(n_{g}<15\right)$, the reduction in device length is already significant compared to the rib waveguide modulator and sub-mm long phase shifter may be achieved. 


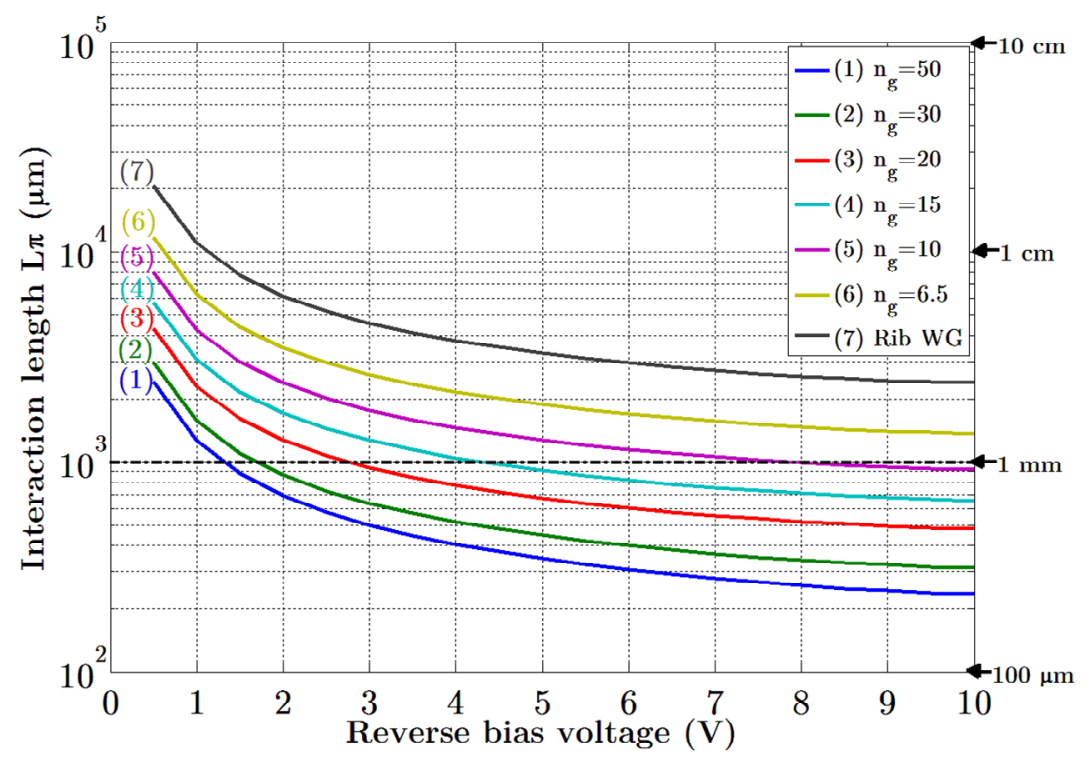

Figure 3-20: Interaction length versus applied reverse bias for varying group index

To complete the analysis, one shall simulate the real device by inserting the SWPS depicted previously in Figure 3-14 into a MZI in order to convert the phase modulation into amplitude modulation as illustrated in Figure 3-21. This SW EOM consists of one $300 \mathrm{~nm}$ wide rib waveguide (reference arm), on one hand and one SWPS on the other hand (active arm). For the sake of clarity, the device is assumed to be lossless and the splitter to divide and recombine the optical power equally.

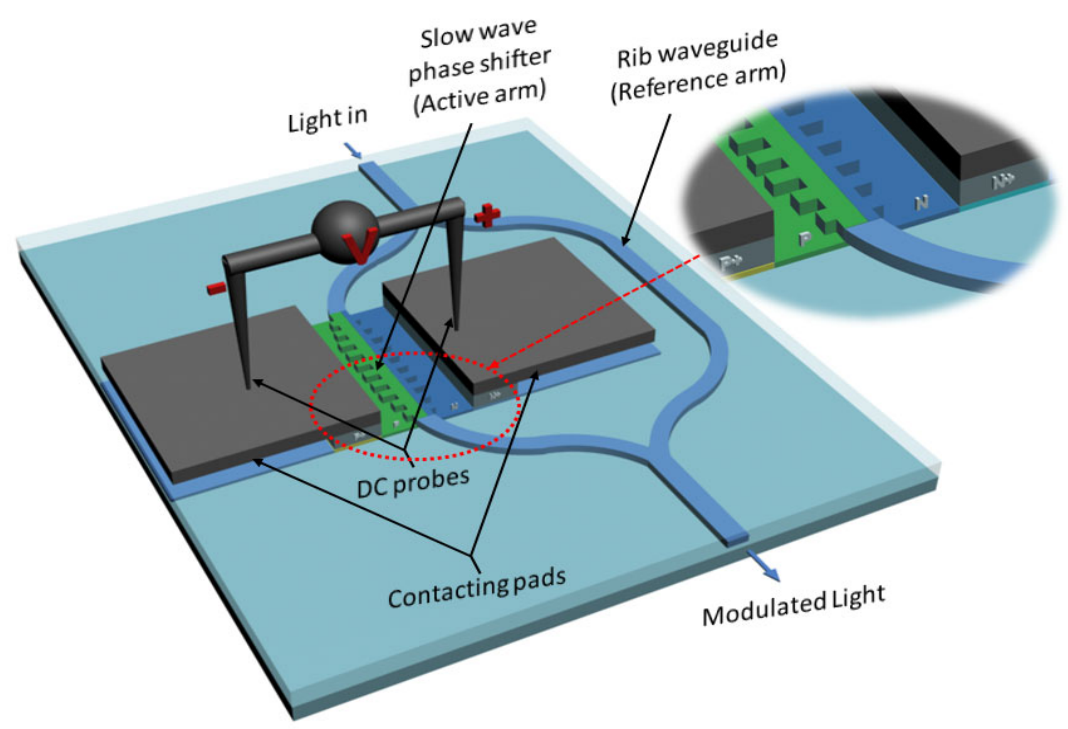

Figure 3-21: Schematic of the reverse biased slow wave single drive amplitude modulator operated in DC. The inset depicts a portion of the phase slow wave shifter. The silicon slab is only represented in the active part of the MZI for clarity. 
When no bias is applied the transmission spectrum of the SW EOM is:

$$
T=\cos ^{2}\left(\frac{\pi}{\lambda} n e f f_{S W P S} \Delta L\right)=\cos ^{2}\left(\frac{\pi}{\lambda} n e f f_{S W P S} L_{S W P S}\right)
$$

Where $n e f f_{S W P S}$ is the effective index of the unbiased SWPS and $\Delta L$ is the optical length difference, i.e. the length of SWPS $\left(\Delta L=L_{S W P S}\right)$

When the phase shifter is reverse biased the transmission spectrum is given by:

$$
T=\cos ^{2}\left(\frac{\pi}{\lambda} L_{S W P S}\left(n e f f_{S W P S}+\Delta n e f f\left(V_{a p p}\right)\right)\right)
$$

Where $\Delta n e f f\left(V_{a p p}\right)$ is the effective index variation within the SWPS as a function of the applied drive voltage.

The resulting transmission spectra of a $500 \mu \mathrm{m}$ long phase shifter for varying group indices illustrated in Figure 3-22 (a) clearly show the potential enhancement in real active devices. In the "fast light" regime (Figure 3-22 (b)), i.e. when the SWPS nearly exhibits a rib waveguide modulator-like behavior, the achieved phase shift for a fixed drive voltage is only a fraction of $\pi(\Delta \Phi=0.17 \pi$ for $n_{g}=3.8$ and $\left.V=-8 \mathrm{~V}\right)$. As the group index increases, i.e. as one enters the slow light regime, the achieved phase shift builds up accordingly. Namely, $\Delta \Phi=0.49 \pi$, $0.95 \pi$, and $1.3 \pi$ for $n_{g}=11,21$ and 30 respectively and $V=-8 \mathrm{~V}$ (resp. Figure 3-22 (b), (c), and (d)). Therefore, for a fixed applied voltage a group index of $\sim 21$, the phase shifter is $\sim 5.5$ times more efficient, meaning that a the SWPS enables the conversion of partial modulation into a complete one, which will eventually improve the ER and/or decrease the interaction length. 


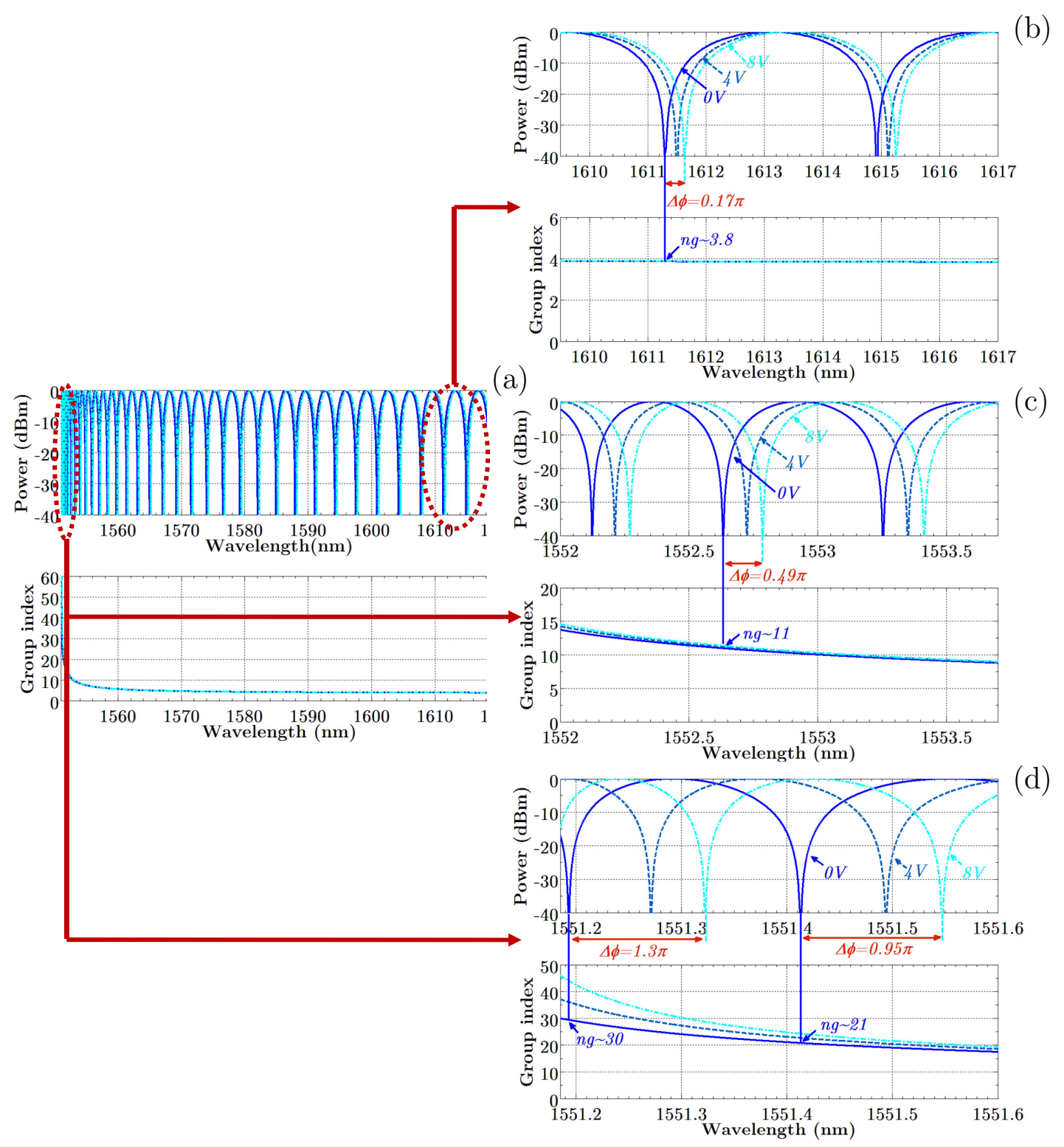

Figure 3-22: Transmission spectra of a Mach-Zehnder modulator (MZM) with a $500 \mu \mathrm{m}-$ long SWPS inserted the active arm for varying applied reverse bias from $V=0 \mathrm{~V}$ (unbiased) to $V=-8 \mathrm{~V}$. (a) depicts the overall transmission spectrum of the MZM. Insets (b), (c), and (d) show portions of spectrum (a) over narrower wavelength ranges to appreciate the phase shift enhancement for increasing group index.

Here, we have illustrated the phase shift enhancement linked to the SWPS for a fixed drive voltage. However, the problem may be tackled the other way around: because partial modulation may even so give rise to a sufficiently high DC ER, say $>7 \mathrm{~dB}$ for practical digital applications, it might be desirable to lower the drive voltage while maintaining the same partial phase shift. For instance, in an effort to reduce cost and space and improve performance, the number of electronic building blocks increases ceaselessly leading to an aggressive 
scaling of the fundamental components, especially transistors. Therefore, due to the reduction in size of the gate oxide, current and future digital logic CMOS technology require much lower drive voltages rounding $1 \mathrm{~V}$. This concretely means that low drive voltages are also highly desirable for optical modulators to ultimately achieve monolithic integration of electronic and photonic components on the same chip. Here, Figure 3-23 shows that that for a low reverse bias, one may achieve large phase shifts and hence high ERs in MZIs consisting of a slightly longer SWPS $(1500 \mu \mathrm{m})$. Indeed, group indices of $\sim 21,26$ and up to 50 enable phase shifts as high as $0.43 \pi, 0.54 \pi$ and $0.97 \pi$ under a DC drive voltage of $1 \mathrm{~V}$.

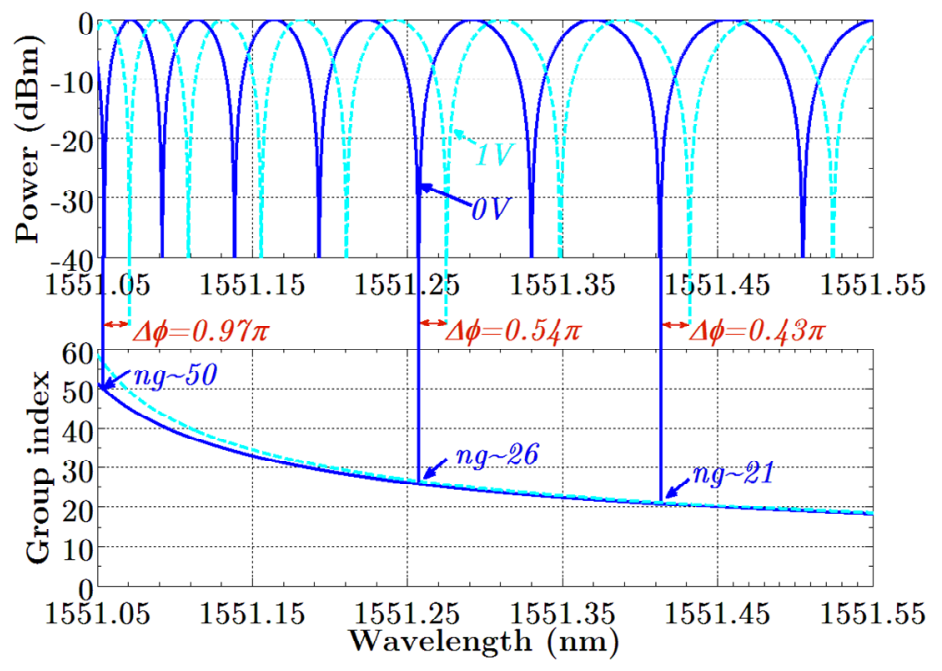

Figure 3-23: Transmission spectrum of a Mach-Zehnder Modulator (MZM) with a 1500 $\mu \mathrm{m}$-long SWPS inserted the active arm for varying applied reverse bias from $V=0 \mathrm{~V}$ (unbiased) to $V=-1 \mathrm{~V}$.

Consequently, because the plasma dispersion effect in silicon turns out to give rise to relatively low effective index change, the need for passive enhancing structures becomes obvious. LCWGs have very advantageous features that may help addressing the challenges and opportunities of silicon optical modulators and silicon components generally speaking.

Overall, a DC analysis has been carried out to estimate the static performance of the modulator. The introduction of a LCWG is shown to drastically enhance the phase shifter performance in terms of effective index change and interaction length. This static performance modeling has to be completed by a transient analysis in order to evaluate the intrinsic high speed modulation capabilities. 


\subsubsection{Transient analysis}

The transient analysis basically consists in applying a linear voltage ramp to the modulator, whose rise and fall time are to be specified as illustrated below.

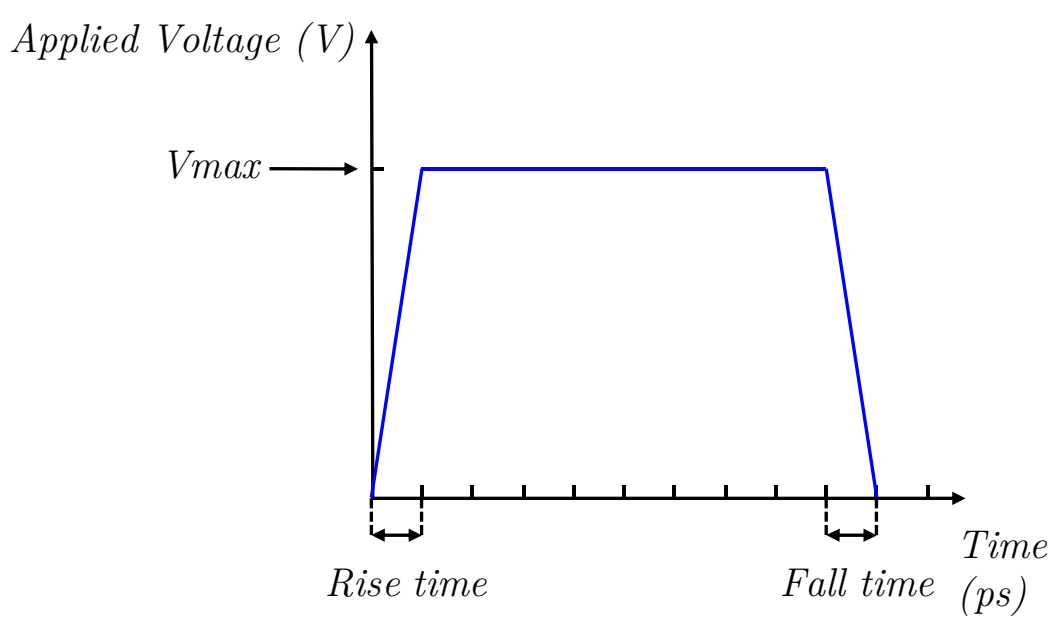

Figure 3-24: Schematic of the ramp excitation applied to the modulator

The applied electric field polarizes the junction following the ramp-like excitation and gives rise to charge carrier motion into the waveguide. The device transient electrical response is computed solving Poisson's equation, carrier continuity equations and transport equations including a set of physical models especially suited for bipolar devices: concentration dependent mobility, field dependent mobility, bandgap narrowing, concentration-dependent lifetime and Auger recombination, etc...

The transient modeling has been realized to work out the intrinsic bandwidth of the SW EOM. A rigorous method consisting in studying the evolution of the effective index as a function of time was followed. To estimate the speed of the modulator, a 1 ps voltage ramp is applied to the electrodes. Although applying tension in such a short time is not practically feasible, this however enables the computation of the intrinsic rise time and fall time of the modulator and therefore its intrinsic bandwidth defined as:

$$
B W=\frac{0.35}{\max \left(t_{\text {rise }}, t_{\text {fall }}\right)}
$$

Where 0.35 is the constant value specific for an RC electrical circuit and $\max \left(t_{\text {rise }}, t_{\text {fall }}\right)$ defines the longest of the two switching times. 
While for the DC analysis, the modal effective index change is worked out as a function of the applied drive voltage (and group velocity) when the junction is in steady state, the transient analysis requires converting the refractive index change matrix into a modal effective index change as a function of time. Thus, electron and hole concentrations are converted into refractive index change matrices by means of Soref's equations. Then, the effective index variations are computed at each time step using the PWE method. The design method flow for the transient analysis of the slow wave modulator is summarized in Figure 3-25.
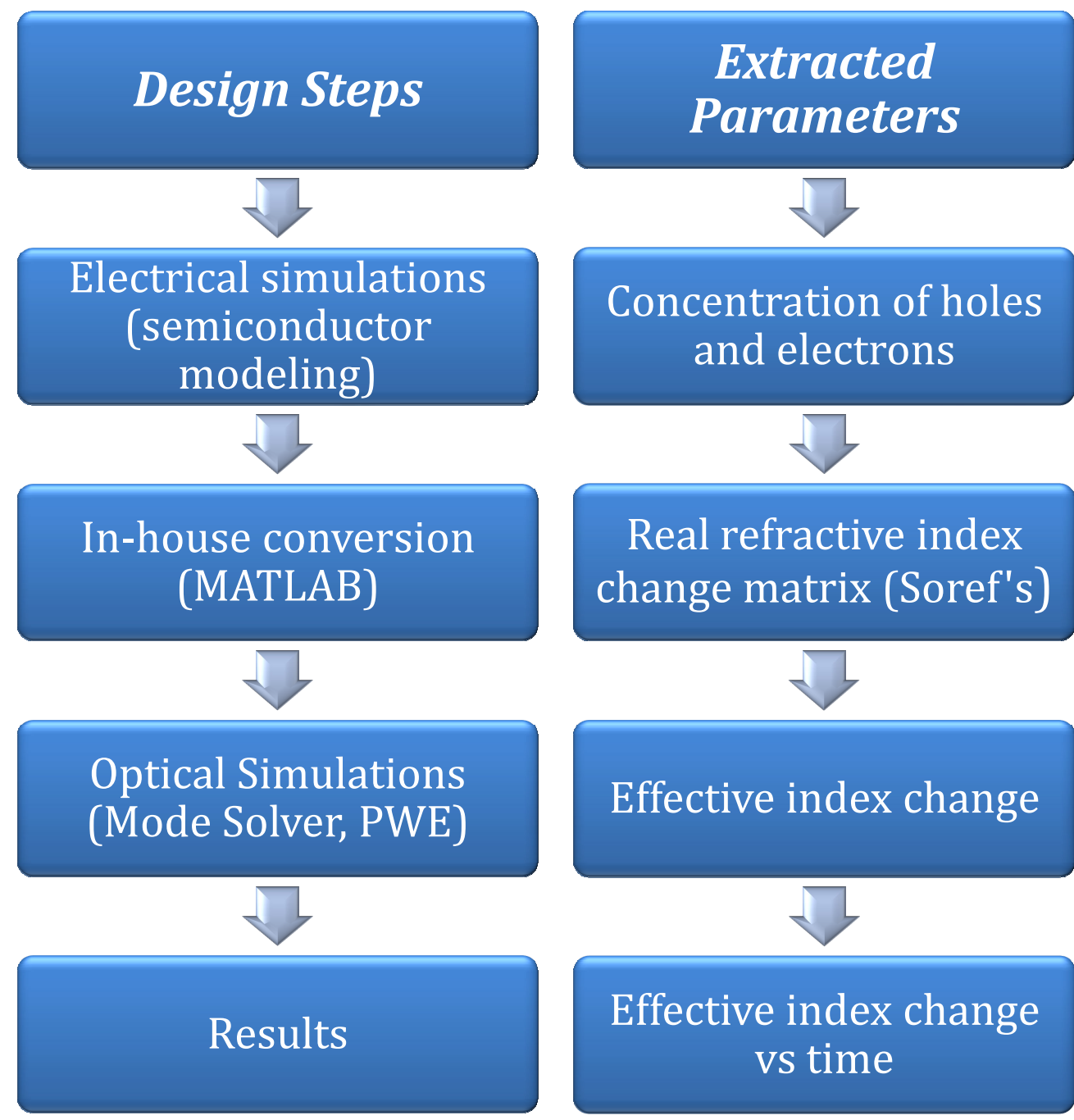

Figure 3-25: Design method flow for the transient analysis of the slow wave modulator 
In this example, the applied drive voltage is set to $V_{\max }=-6 \mathrm{~V}$ and both rise and fall time to 1 ps as previously mentioned. Figure 3-26 shows the refractive index change as a function of time. To ensure the phase shifter reaches its steady state (just as in DC), the junction is polarized over a duration of $100 \mathrm{ps}$ and released over a duration of $100 \mathrm{ps}$, leading to a total time interval of $200 \mathrm{ps}$. Here again, the effective index change enhancement compared to a rib waveguide phase shifter is straightforward. The rise time is defined as time needed to reach $90 \%$ of the maximum effective index change in DC. In this case, the $p n$ junction is reversed biased. Then, the fall time is defined as the time needed for effective index change to fall down to $10 \%$ of its maximum value after the $p n$ junction is released.

The intrinsic rise and fall times are both $t_{\text {rise }}=t_{\text {fall }}=20 \mathrm{ps}$, respectively for all considered group velocities. Using Equation (4.10), the intrinsic modulation bandwidth is therefore slightly higher than $17 \mathrm{GHz}$.

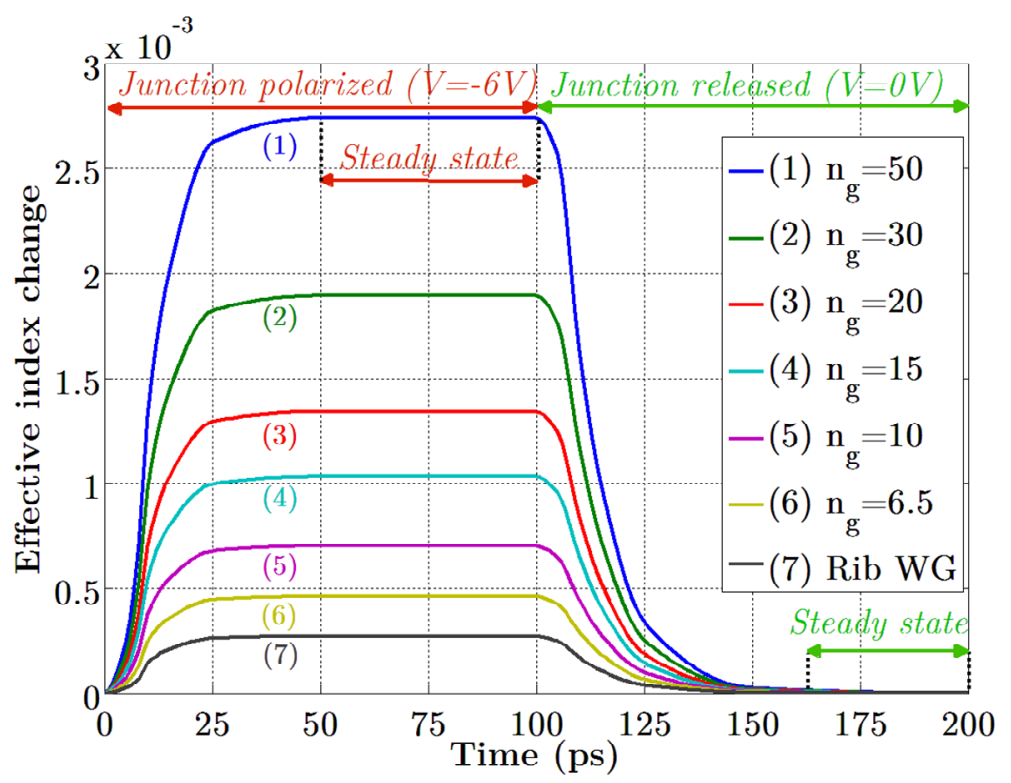

Figure 3-26: Transient response of the slow wave phase shifter: Effective index change versus time for varying group index under an applied voltage $V_{m a x}=-6 \mathrm{~V}$.

To complete the study, one may also extract on one hand the maximum effective index change and resulting interaction length against group index as depicted in Figure 3-27. The drastic reduction in the interaction length is clearly pictured here, confirming that for even moderate group indices $10<n_{g}<15$, submm phase shifters are achievable. 


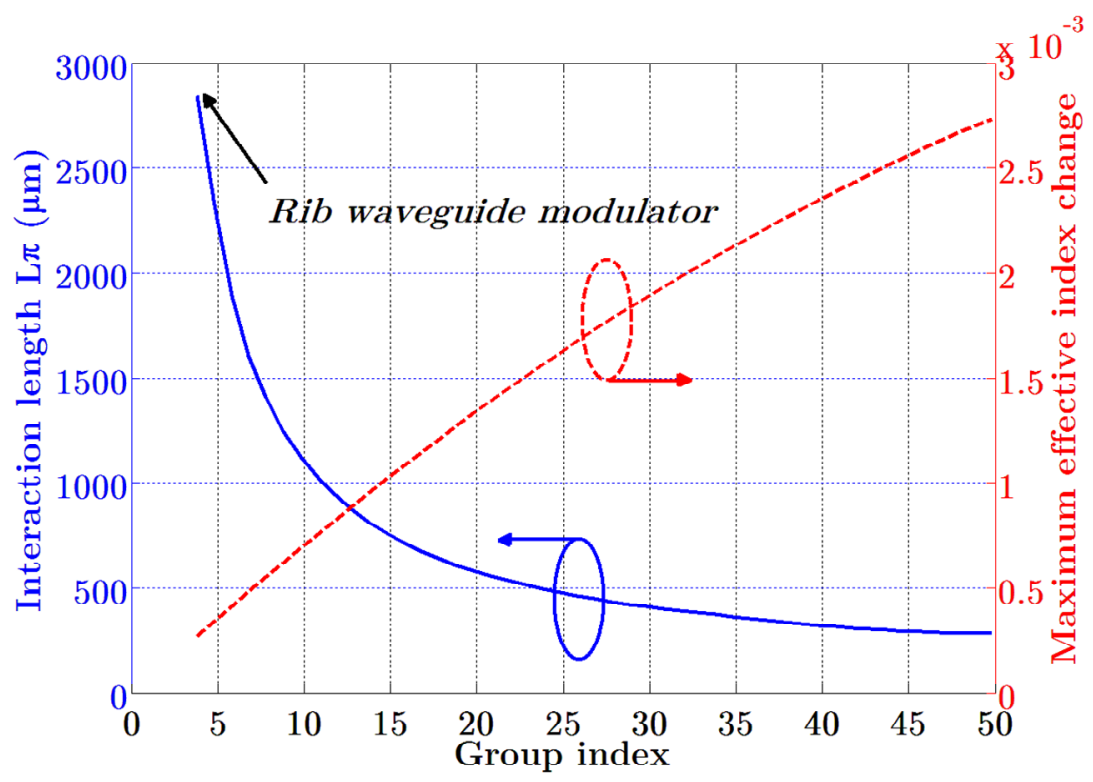

Figure 3-27: Maximum effective index change (red curve) and resulting interaction length (blue curve) against group index for $V_{\max }=-6 \mathrm{~V}$.

Likewise, one may also represent the specific figure of merit $\left(V_{\pi} L_{\pi}\right)$ of this slow wave phase shifter as a function of the group index Figure 3-28. Again, the figure of merit is drastically reduced as the group index increases reaching subV.cm for moderate group indices $7<n_{g}<15$.

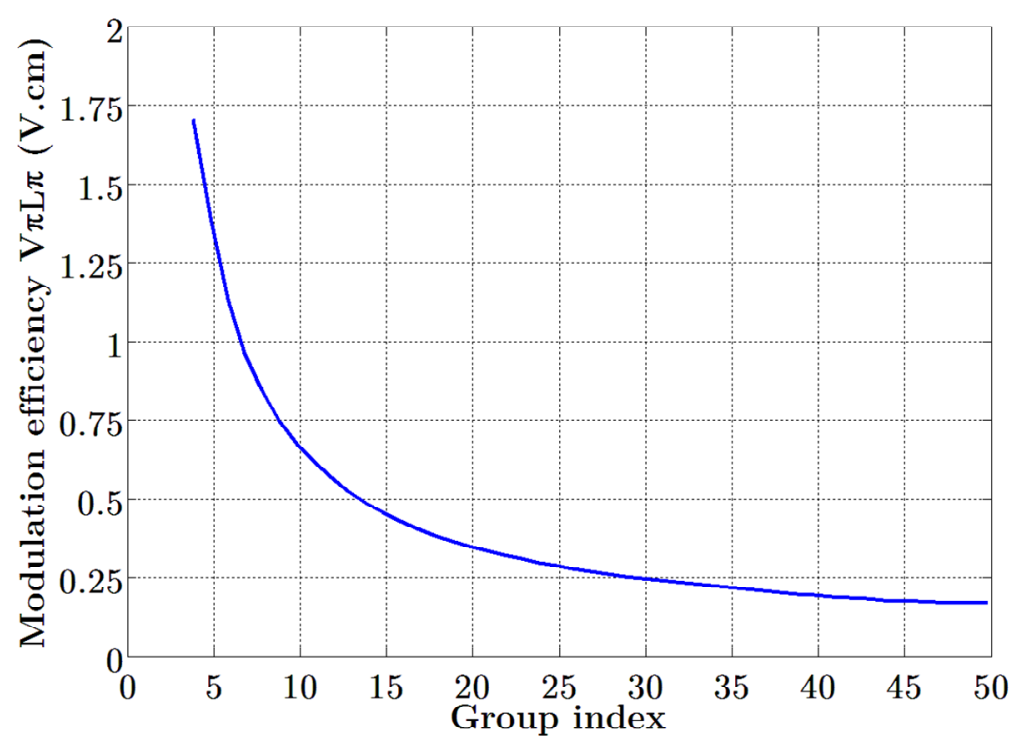

Figure 3-28: Figure of merit of the slow wave phase shifter versus group index for a $V_{\max }=-6 \mathrm{~V}$ reverve bias voltage.

Overall it has been shown that LCWGs are poised to strongly enhance the performance of conventional rib waveguide modulators. The example of a LCWG embedded into an asymmetric pn junction has been presented and complete 
analysis in both DC and transient regimes has been carried out. The slow wave modulator features simultaneously high intrinsic bandwidth $(17 \mathrm{GHz})$ and high ER over sub-mm lengths even for low drive voltages.

Nevertheless, such slow light enhancement hides major challenges due to low group velocity, namely the propagation losses due to enhanced interaction with sidewall roughness and absorbing charges carriers as well as narrow useful optical bandwidth. The following sections are devoted to the analysis of both potential issues.

\subsubsection{Analysis of slow light issues}

(a) Loss: In the previous section, slow light has been shown to potentially enhance the performance of EOMs through a concrete example. However, as already mentioned in section 3.3.3, losses linked to slow wave propagation might be a serious issue for practical devices and low loss applications in general. Models and experiments have been developed to describe the loss mechanisms in $\mathrm{PhCs}$ and their dependency on the group index [90, 106-108]. Notwithstanding, it remains difficult to clearly identify their sources and estimate to what extent they may affect a real device.

Here, two sources of losses in the slow light regime are foreseen: increased absorption due to enhanced optical mode interaction with charge carriers, i.e. free carrier absorption loss (FCAL) as well as scattering due to enhanced optical mode interaction with sidewall roughness, i.e. sidewall roughness scattering loss (SRSL).

Because one intends to operate the EOM in the moderate slow light regime $\left(n_{g}<25\right)$ and in accordance with the majority of high impact publications, [105108], the scaling factor is assumed to be $n_{g}=1 / v_{g}$ for both abovementioned sources of losses. It follows that the total insertion losses (IL) in $\mathrm{dB} / \mathrm{mm}$ may be given by the following Equation (4.11).

$$
\begin{gathered}
I L(d B / m m)=F C A L+S R S L \\
=-(\underbrace{10 \log _{10} \exp \left(-\alpha_{\text {carriers }} n_{g}\right)}_{\text {free carrier absorption }}+\underbrace{10 \log _{10} \exp \left(-\alpha_{\text {roughness }} n_{g}\right)}_{\text {Sidewall roughness scattering }})
\end{gathered}
$$

Where $\boldsymbol{\alpha}_{\text {carriers }}$ and $\boldsymbol{\alpha}_{\text {roughness }}$ are the absorption coefficients due to charge carriers and sidewall roughness, respectively $\left(\right.$ in $\mathrm{mm}^{-1}$ ). 
The carrier absorption coefficients $\boldsymbol{\alpha}_{\text {carriers }}$ for varying applied voltage is then calculated through Soref's expression in a rib waveguide of dimensions identical to the thin section of the LCWG (Table 3-6):

$$
\alpha_{\text {carriers }}(V)=\alpha_{e}(V)+\alpha_{h}(V)=8 \cdot 5 \cdot 10^{-18} N_{e}(V)+6.10^{-18} N_{h}(V)
$$

Where $\alpha_{e}$ and $\alpha_{h}$ are the absorption coefficients of electrons and holes, respectively and $N_{e}$ and $N_{h}$ are the electrons and holes concentrations, respectively.

\begin{tabular}{|c|c|}
\hline Applied voltage $(\boldsymbol{V})$ & $\boldsymbol{\alpha}_{\text {carriers }}\left(\mathbf{m m}^{-1}\right)$ \\
\hline 0 & 0,019380 \\
\hline-2 & 0,015880 \\
\hline-4 & 0,010960 \\
\hline-6 & 0,007704 \\
\hline-8 & 0,005305 \\
\hline-10 & 0,004069 \\
\hline
\end{tabular}

Table 3-6: Absorption coefficient due to charge carriers as calculated from BPM simulations in a rib waveguide of dimensions $W=300 \mathrm{~nm} \times H=220$ and slab height $=50$ $\mathrm{nm}$

As expected, the absorption coefficient due to charge carrier decreases as the applied voltage increases. This is because as the reverse bias voltage builds up, an increasing number of free carriers are swept out of the active region, which is also the waveguiding region. As a result, the absorption decreases. FCAL is then worked out using Equation (4.11) for each reverse bias voltage and depicted in Figure 3-29.

Moreover, SRCL have been set at $0.3 \mathrm{~dB} / \mathrm{mm}$, in accordance with standard low loss waveguides fabricated with deep-UV lithography [6]. The corresponding absorption coefficient $\alpha_{\text {roughness }}=0.0691 \mathrm{~mm}^{-1}$ is then introduced into Equation (4.11) to estimate the SRCL as shown in Figure 3-30. 


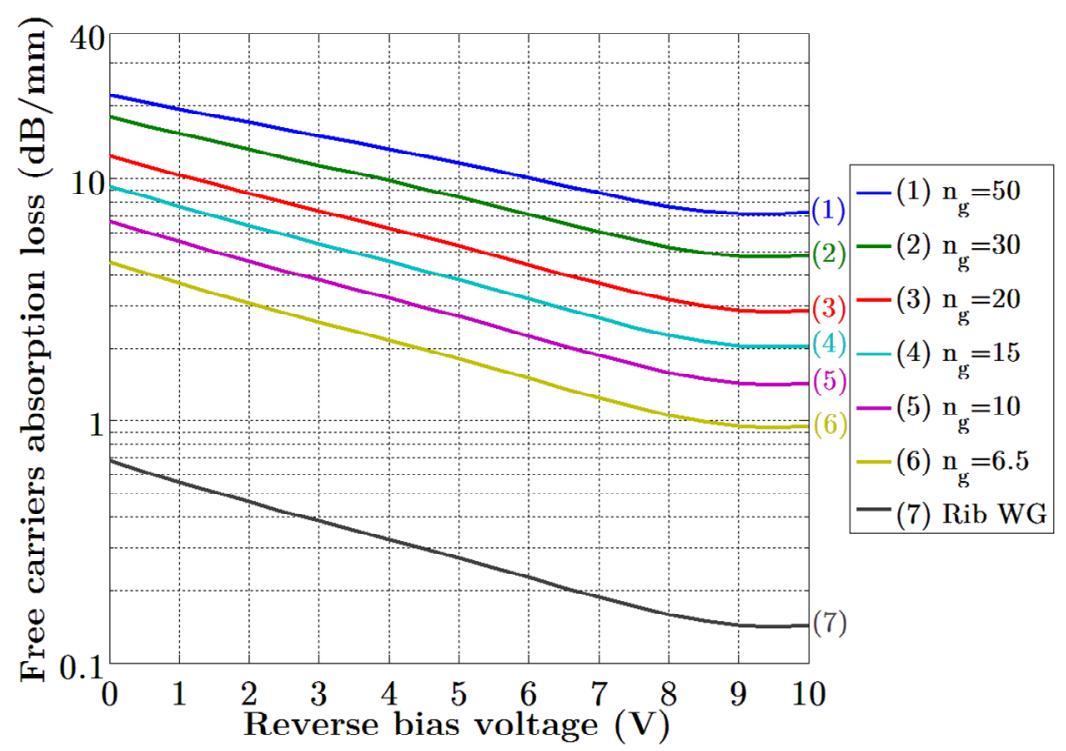

Figure 3-29: Free carrier absorption loss versus applied reverse bias voltage for varying group index

After Figure 3-29 and Figure 3-30, in contrast with the FCAL, which decreases clearly as the reverse bias voltage increases, SRSL increases as the reverse bias voltage does. In both cases, the loss figure variation is all the more high that the group index is itself high. This is because for a fixed wavelength, as we tune the MZI transmission spectra the group index is altered accordingly. The intensity of the variation depends on the initial group index (which is in fact $\left.n_{g}\right)$ i.e. when the modulator is unbiased as previously seen in Figure 3-22. Indeed, the higher the initial group index $\left(n_{g}\right)$, the stronger the group index variation $\Delta n_{g}$ $=n_{g}$ (biased) $-n_{g}$ (unbiased) due to the steep curve slope at higher group index (as exposed thereupon in Figure 3-31). Consequently, operating at higher group index $\left(n_{g}>10\right)$, and high voltage may seriously affect the phase shifter transmission, and hence the single drive MZI performance due to the power asymmetry between the active and reference arm. 


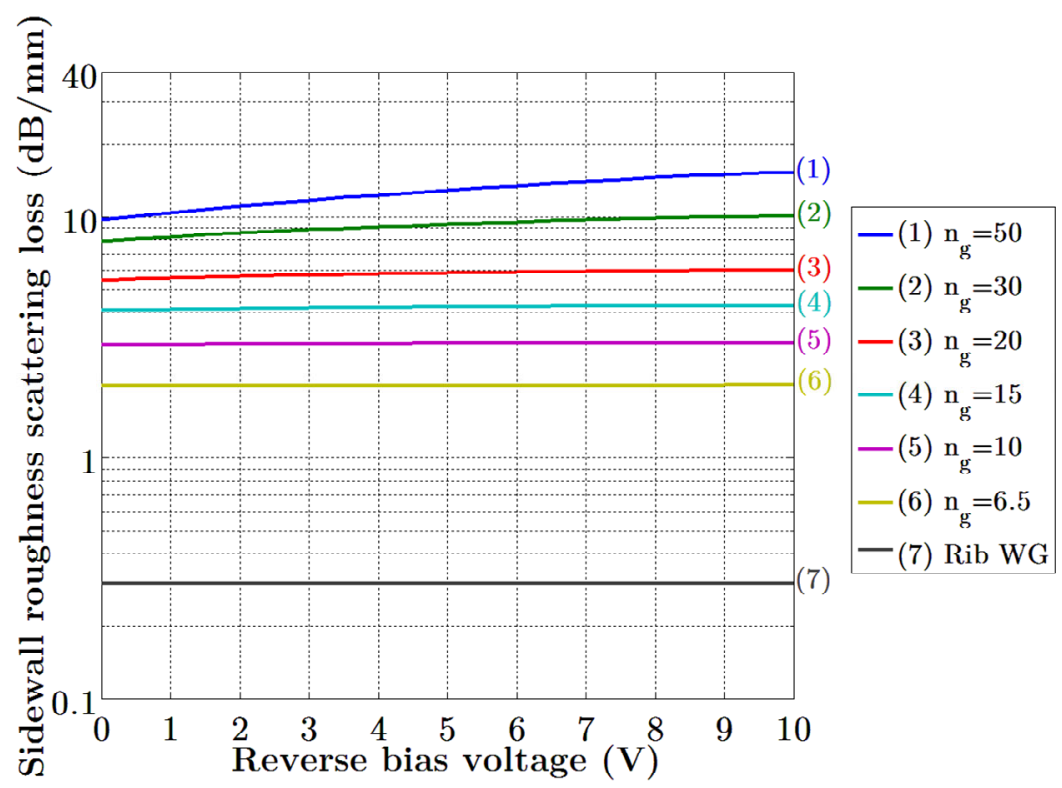

Figure 3-30: Scattering loss due to sidewall roughness versus applied reverse bias voltage for varying group index.

However, it may be pointed out that for higher group indices, the SRSL increases as the reverse bias voltage does in contrast to the FCAL. This is due to the fact that since the group index increases for increasing reverse bias, scattering losses due sidewall roughness enlarges accordingly. The total IL, corresponding to the sum of the SRCL and FCAL as highlighted in Equation (4.11), is shown in Figure $3-32$.

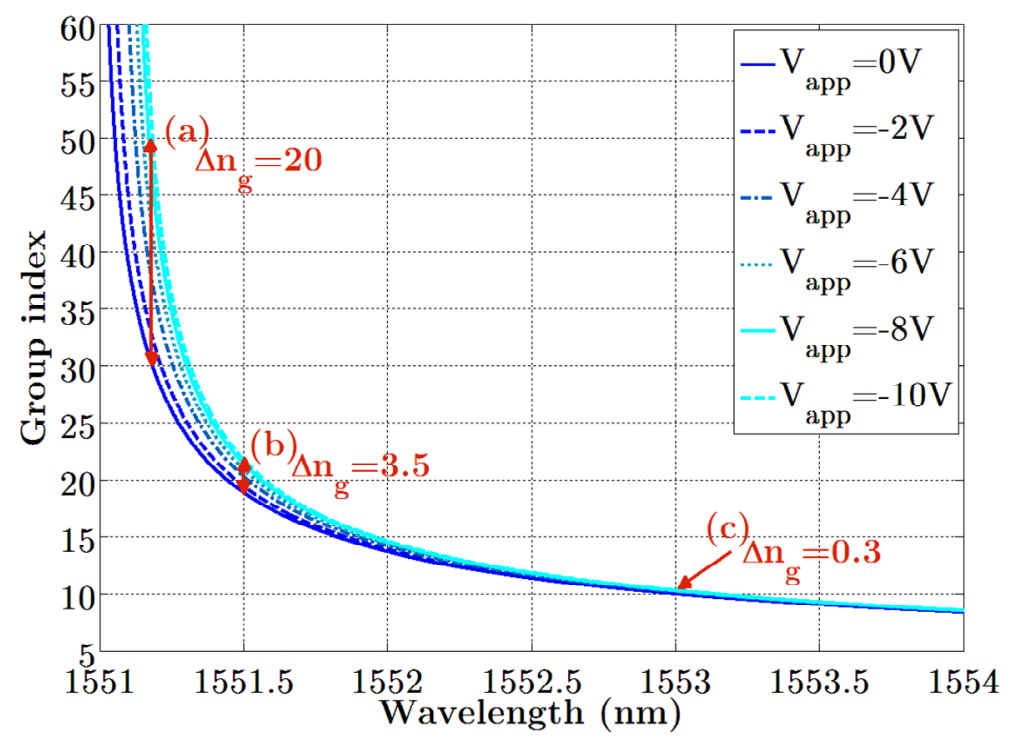

Figure 3-31: Group index versus wavelength for varying applied reverse bias voltage. (a), (b), (c), depict some examples of the group index variation $\Delta n_{g}=n_{g}$ (bias)- $n_{g}$ (unbiased) where $n_{g}$ (unbiased) $=30,20$, and 10 , respectively. 


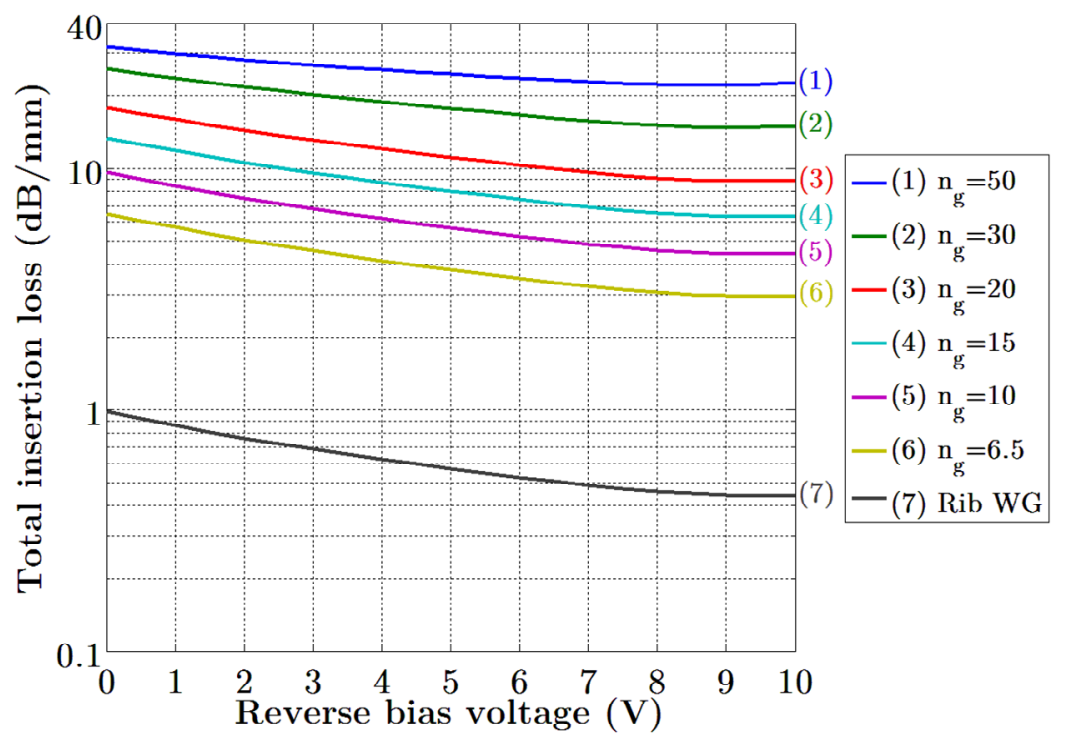

Figure 3-32: Total insertion loss versus applied reverse bias voltage for varying group index

As expected, high group indices are responsible for relatively high insertion loss per unit distance. Notwithstanding, as discussed in section 3.4.2, the fact that slower modes interact more strongly with the free carrier plasma offers three design alternatives over conventional rib waveguide EOMs, namely:

\section{Shorter device operated under the same applied drive voltage \\ 2. Lower drive voltage while conserving the same device length \\ 3. A combination of both abovementioned alternatives}

The first design alternative should be adopted when the conventional rib waveguide EOM exhibits a sufficiently high ER when operated in DC but over a length that may be a significant hurdle for high speed operation. Indeed, because conventional MZIs require long $p n$ junctions (on the order of several $\mathrm{mm}$ ) to achieve decent phase shifts and ERs, the overall performance may be impaired by CPWG RF losses, as well as the higher influence of the velocity mismatch between the electrical and optical signals (where appropriate). Moreover, as stated before, a large device footprint is not desirable. From and below $1 \mathrm{~mm}$ interaction length, these issues may be partially resolved, leading eventually to higher modulation speeds but at the expense of lower phase shifts and ERs [19]. As a result, the objective of using LCWGs is hence to achieve the same or higher phase shifts over much shorter lengths. 
The second design alternative should be picked out on one hand, when the rib waveguide EOM features a low ER even under DC operation. The target of LCWGs would be hence to sustain the phase shifter length in order to enhance the phase shift and ER, accordingly. On the other hand, when the rib waveguide EOM presents a sufficiently high ER, but has to be operated under lower voltage.

The third design alternative should be selected depending on the design specifications. In any case, a trade-off should be established so as to define what level of performance is achieved in rib waveguide modulators and to what extent introducing LCWGs would improve the overall performance without introducing excessive loss.

To illustrate this alternative through concrete examples, we refer back to the design of the SWPS. If the design target is to achieve:

"1. Shorter device operated under the same applied drive voltage (as for rib waveguide EOM)"

According to Figure 3-33, the achieved phase shift in a $1 \mathrm{~mm}$ long rib waveguide EOM and $V a p p=-6 \mathrm{~V}$ is approximately $\Delta \Phi=0.337 \pi$. If one wishes to maintain the same phase shift in a SWPS designed to exhibit a moderate group index $n_{g}=10$, the device length may be reduced down to $387 \mu \mathrm{m}$, suggesting a clear footprint reduction. The associated maximum SWPS loss scales reasonably from $1 \mathrm{~dB}$ (rib waveguide) to $3.7 \mathrm{~dB}$ as shown in Figure 3-34. For higher group indices, say $n_{g}=20$, the device length may be reduced down to $204 \mu \mathrm{m}$, and the maximum phase shifter loss would be only $3.64 \mathrm{~dB}$. This alternative may therefore be of interest as even for moderate group index one can achieve a strong device footprint reduction with moderate insertion loss penalty, which moreover, is nearly independent on the considered group index. 


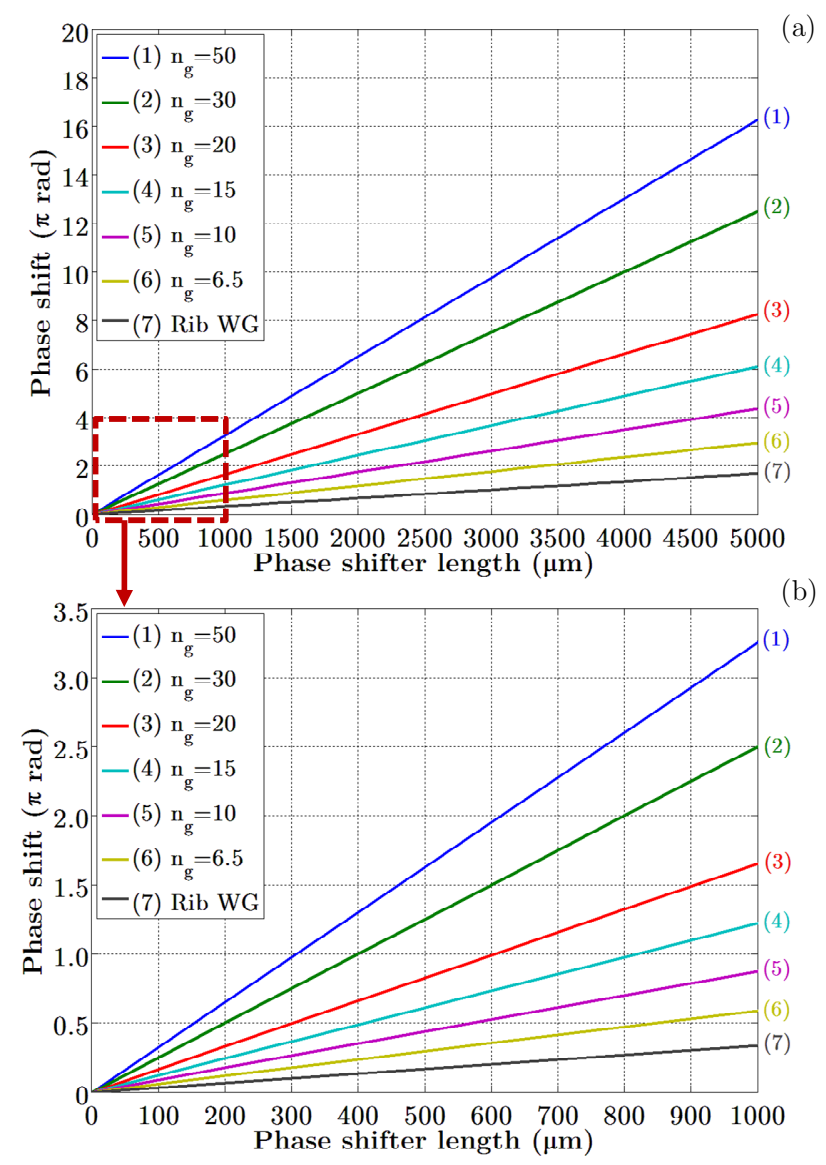

Figure 3-33: (a) Achieved phase shift versus SWPS length for a -6V reverse bias voltage. Inset (b) shows an enlarged view of the region surrounded by a dashed rectangle.

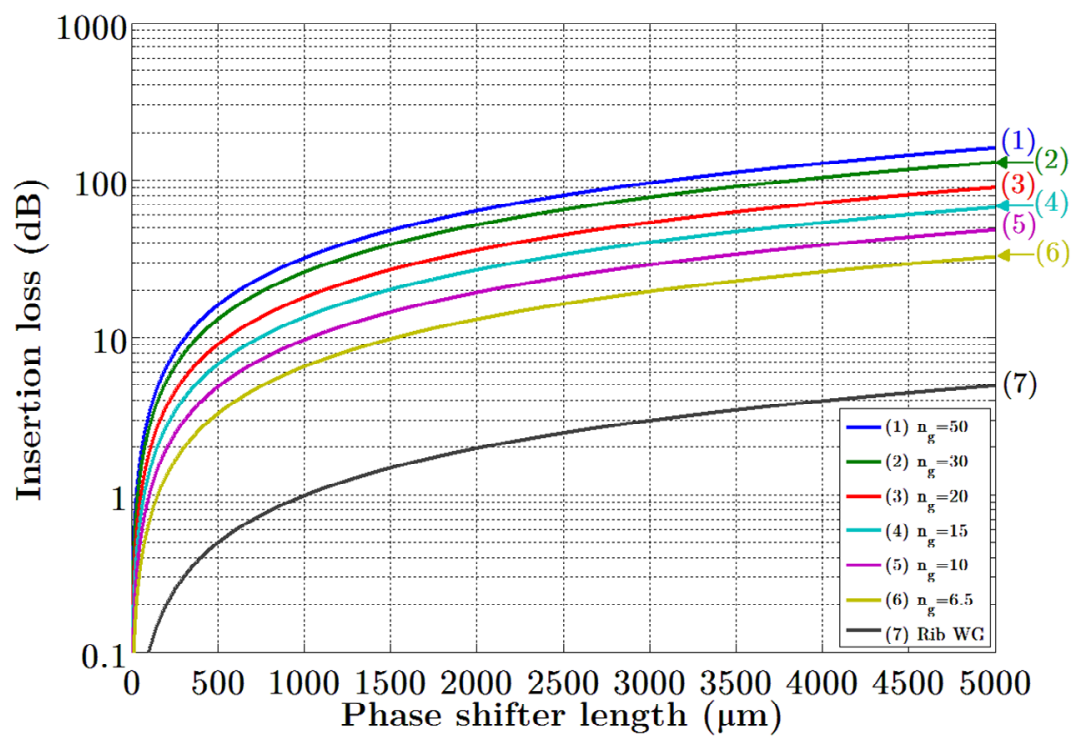

Figure 3-34: Phase shifter loss versus phase shifter length for varying group index assuming a $-6 \mathrm{~V}$ reverse bias voltage 
"2. Lower drive voltage while conserving the same device length (as for rib waveguide EOM)"

According to Figure 3-35, the achieved phase shift in a $1 \mathrm{~mm}$ long rib waveguide EOM and $V a p p=-6 \mathrm{~V}$ is approximately $\Delta \Phi=0.337 \pi$. If one wishes to maintain the same interaction length $(1 \mathrm{~mm})$ in a SWPS designed to exhibit a moderate group index $n_{g}=10$, the required drive voltage may be lowered down to $-1.52 \mathrm{~V}$. The associated maximum phase shifter loss scales however from $1 \mathrm{~dB}$ (rib waveguide) to $7.9 \mathrm{~dB}$. For higher group index, say $n_{g}=20$, the required drive voltage may be decreased down to $-0.76 \mathrm{~V}$, and the maximum SWPS loss would be $16.4 \mathrm{~dB}$. Because the latter values are clearly too high for practical devices, one may consider lower group indices such as $n_{g}=6.5$. In this case, the required drive voltage would be decreased down to $-2.5 \mathrm{~V}$, with associated SWPS loss of about $4.8 \mathrm{~dB}$. This shows that one may achieve low drive voltage with relatively low loss.

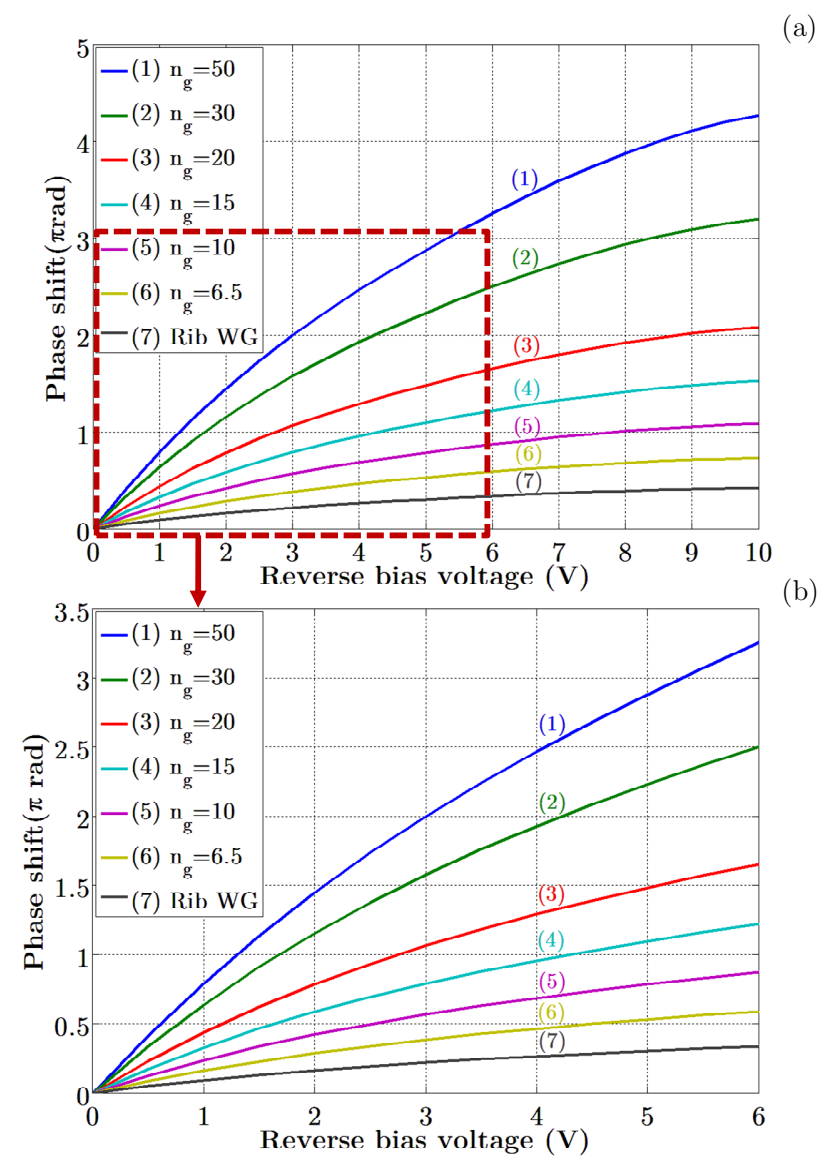

Figure 3-35: (a) Achieved phase shift in a $1 \mathrm{~mm}$ long phase shifter versus applied drive voltage. Inset (b) shows zoomed in view of the region surrounded by a dashed rectangle. 


\section{"3. A combination of both above mentioned alternatives"}

Because the SWPS insertion loss and/or length and/or drive voltage may exceed acceptable values, a trade-off between these three key features may be set. As an example, it was shown that the SWPS designed for a moderate group index $n_{g}=10$, can achieve a length reduction down to $387 \mu \mathrm{m}$, for a $-6 \mathrm{~V}$ applied voltage and $3.7 \mathrm{~dB}$ insertion loss. To address the third design alternative, the drive voltage may be readily decreased to $-3 \mathrm{~V}$, at the expense of a twice longer SWPS $(774 \mu \mathrm{m})$ and insertion loss $(7.4 \mathrm{~dB})$.

Overall, it was shown that even moderate group index may readily enhance the performance of a MZI EOM either by decreasing the device length or the required drive voltage, or a combination of both at the expense of higher, although moderate insertion loss. This suggests the SWPS design, due to inherent degrees of freedom, is subject to discussion. The questions remain as whether this SWPS can meet the specifications of practical nanophotonic circuits and how tight the key parameters may be adjusted.

(b) Optical bandwidth: Another critical point linked to LCWGs and slow wave structures in general is the optical bandwidth, that is the frequency (respectively wavelength) range over which the "enhancement on-target" remains valid. Although there is no universal definition of such a parameter, which once again depends on the required specifications, we define the optical bandwidth in accordance with literature references $[83,94,96,116]$ as the range frequencies (respectively wavelength) over which the group index is constant within $\pm 10 \%$ of the designed value. For the sake of comparison, the optical bandwidth of the LCWG is related to the quality factor of rings resonators defined as $\lambda / \Delta \lambda$ at $-3 \mathrm{~dB}$ as depicted in Figure 3-36. 


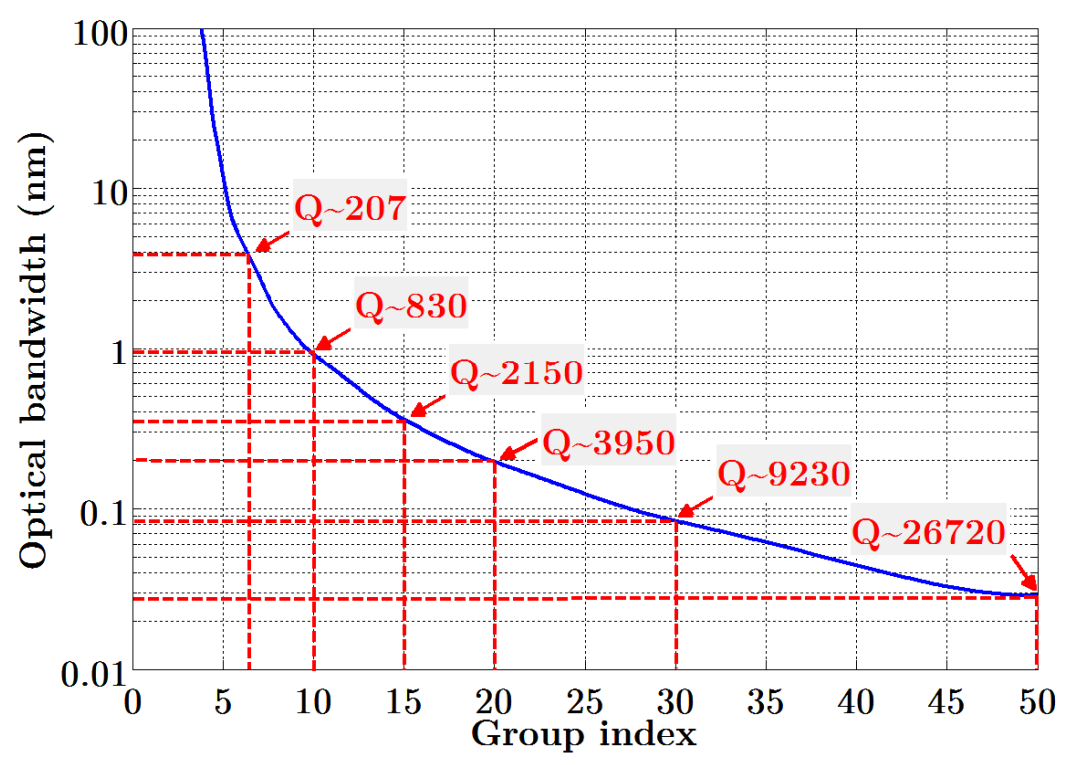

Figure 3-36: Optical bandwidth (after references [83, 94, 96, 116]) versus group index for a LCWG. Q factor equivalents of ring resonators are indicated for the sake of comparison.

The optical bandwidth clearly decreases as the group index $\left(n_{g}\right)$ increases, uncovering hence the main limitation of such a slow wave structure for broad optical bandwidth applications. To illustrate such an issue, we consider the case of a symmetric MZI with two identical SWPS in both arms as illustrated below. The transmission of such a device is given by:

$$
T(\lambda)=\cos ^{2}\left(\frac{\pi}{\lambda} \Delta n e f f\left(V_{a p p}\right) L\right)
$$

If no voltage is applied to the SWPS, i.e. $\Delta \operatorname{neff}\left(V_{a p p}\right)=0$, the transmission is $\mathrm{T}=1$ and flat over the considered wavelength range. The SW EOM is in the "on-state". Then, as we apply a voltage on one of the SWPS (here, we assume that the modulator is single drive for the sake of simplicity), the MZI response spectrum exhibit notches where transmission comes close to zero. Assuming a fixed effective index change for a given applied voltage, the position of the notch may be close to the wavelength of interest by properly selecting the optimum SWPS length $\left(L_{\text {opt }}\right)$ as:

$$
L_{o p t}=\cos ^{-1}(\sqrt{T}) \frac{\lambda_{0}}{\pi \Delta n e f f}
$$


This optimum SWPS length may be selected depending on the desired ER. For instance, if a given $\mathrm{ER}\left(\mathrm{ER}(\mathrm{dB})=T_{o n}-T_{\text {off }}\right)$ is satisfactory for the target application, then the optimum length might be selected accordingly as pictured in Figure 3-37. Here, we assume the SW EOM is operated at $n_{g}=10$ at $\lambda_{0}=1.553 \mu \mathrm{m}$ and for a $\mathrm{V}_{a p p}=-10 \mathrm{~V}$. The ER "on-target" is $>30 \mathrm{~dB}\left(T_{o f f}=0.001\right)$ and the optimum SWPS length is therefore $L_{o p t}=846 \mu \mathrm{m}$. Referring back to Figure 3-32, the maximum IL for this SWPS would be $8.1 \mathrm{~dB}$ (at 0V).

Figure 3-38 illustrates the SW EOM response as a function of the applied voltage. Both SWPS have identical length $L_{o p t}=846 \mu \mathrm{m}$ and therefore similar IL, to avoid ER degradation. It is clear from Figure 3-38 that the notch, i.e. the near "zero transmission" point is located just at the operating wavelength $\lambda_{0}=1.553 \mu \mathrm{m}$ (i.e. corresponding to the designed group index $n_{g}=10$ ) for a $-10 \mathrm{~V}$ applied voltage, confirming $L_{o p t}=846 \mu \mathrm{m}$ as being optimum.

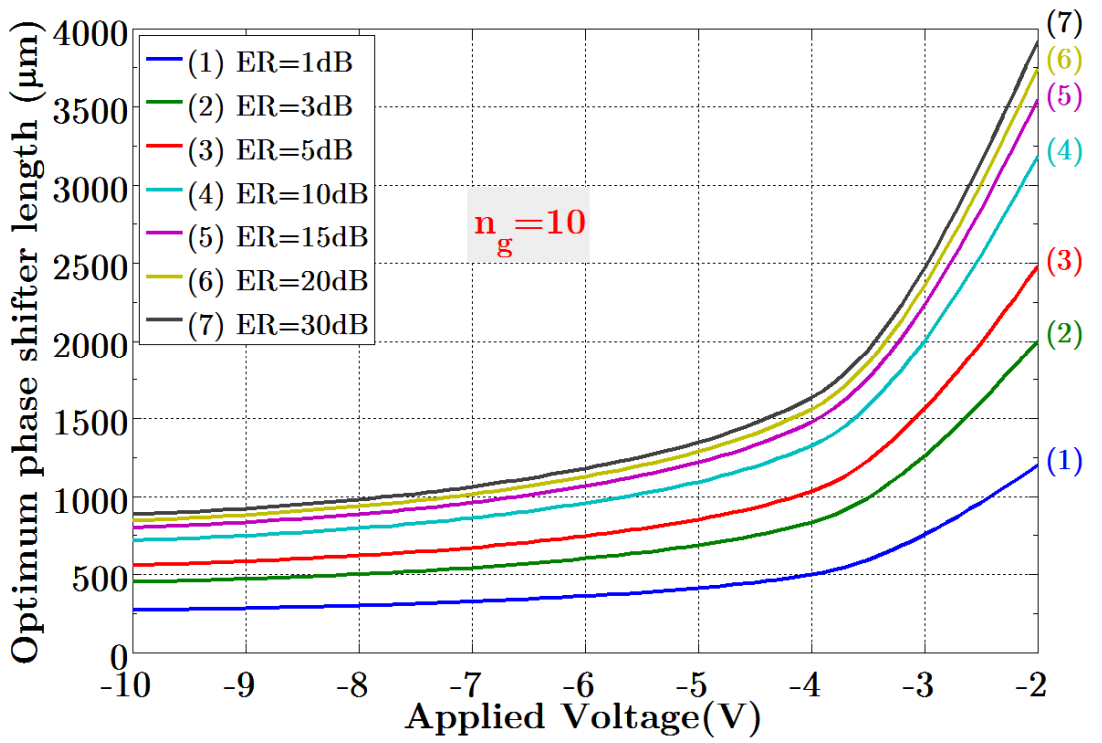

Figure 3-37: Optimum SWPS length versus applied voltage for varying ER "on-target". A group index $n_{g}=10$ is assumed.

On the other hand, a close look at Figure 3-38 clearly shows that the optical bandwidth of such a device depends on how tight the final device specifications are. In other words, the extinction ER "on-target" sets the optical bandwidth over which the device performance fits the specifications. 


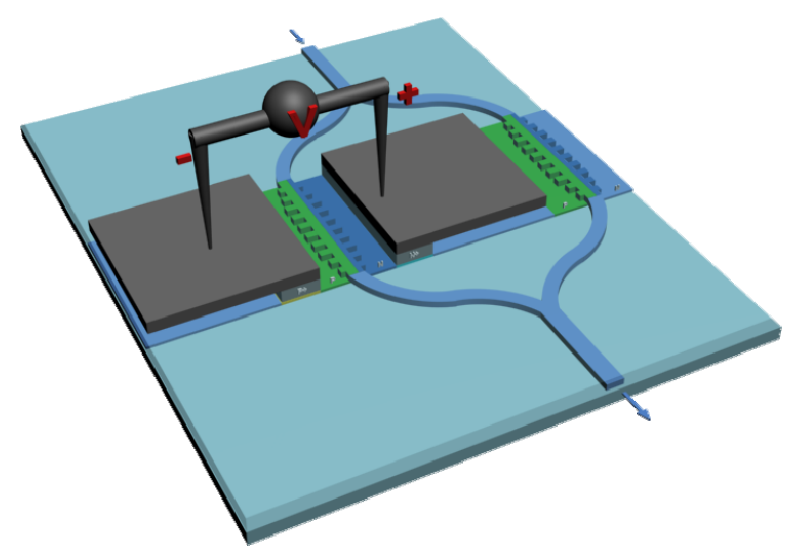

(a)
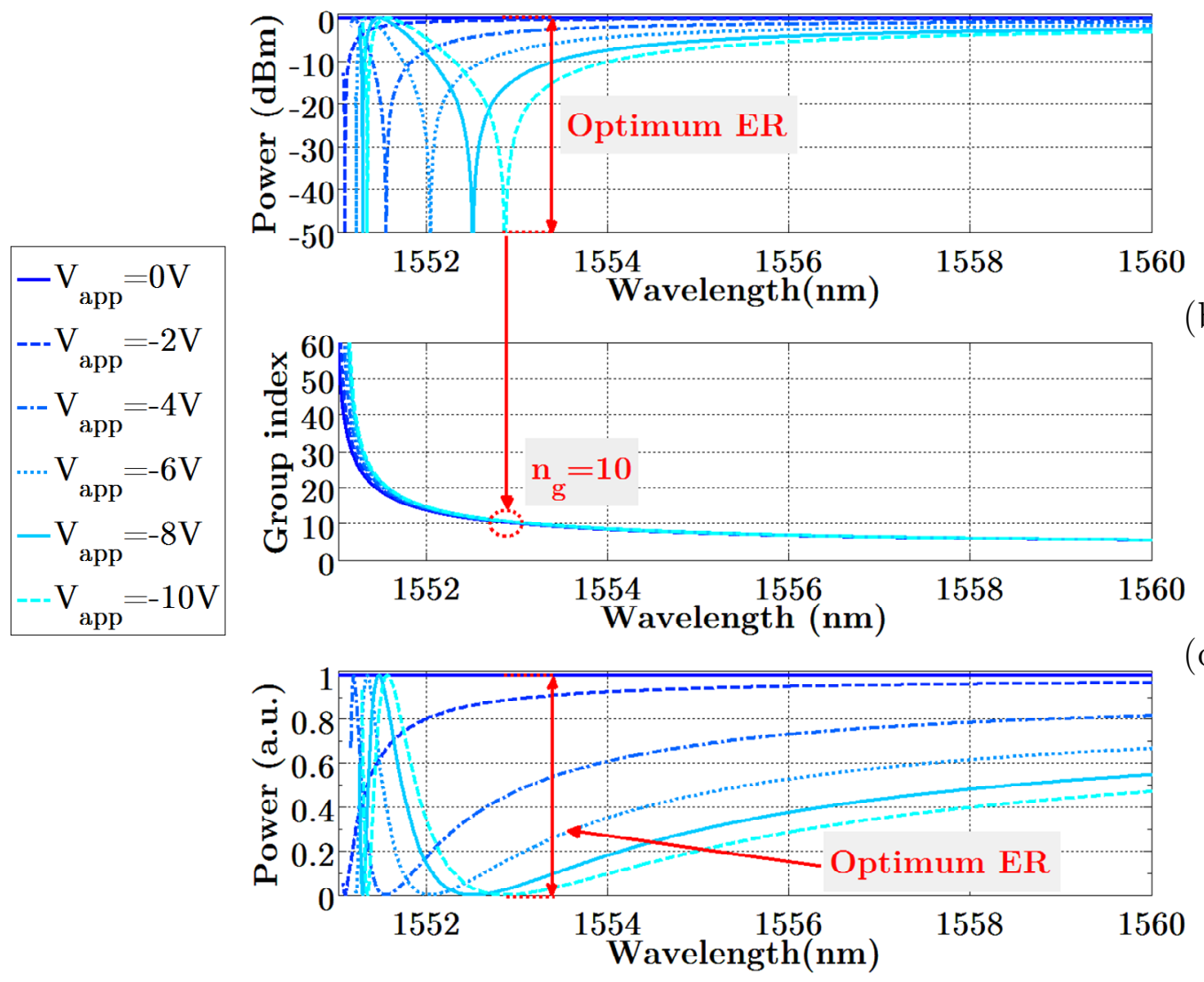

(b)

(c)

Figure 3-38: (a)Logarithmic (dBm), (b) group index versus wavelength and (c) linear (a.u.) response of a symmetric MZI with identical SWPS integrated in both arms to balance losses. A group index $n_{g}=10$ is assumed.

Additionally, one may extract the tolerable group index variation (\%) as a function of the minimum tolerable ER (worst case scenario) as well as the optical bandwidth as a function of the ER "on target" as depicted in Figure 3-39 and Figure 3-40, respectively. As expected, the tolerance on the group index variation becomes more severe as the minimum bearable ER increases. As an example, maintaining a group index tolerance of $\mathrm{x} \%$ around the target value means 
altering the performance of the modulator through the reduction of the maximum ER value down to a lower ER. Namely, setting the minimum tolerable ER at 15 $\mathrm{dB}$ causes the acceptable group index variations to lie in a $6.0 \%, 13.2 \%, 17.3 \%$, $21.0 \%$ and $22.0 \%$ range for design group indices of 30, 20, 15, 10, 6.5 respectively. If one takes the problem the other way around, i.e. one wishes to maintain a $10 \%$ group index variation, (as usually found in the literature [83, 94]) in the worst case scenario the ER would be $11 \mathrm{~dB}, 17.3 \mathrm{~dB}, 19.7 \mathrm{~dB}, 21.3 \mathrm{~dB}$ y $22 \mathrm{~dB}$ for group indices of $30,20,15,10,6.5$ respectively. This suggests that the performance of the SW EOM may be affected more easily at higher group index.

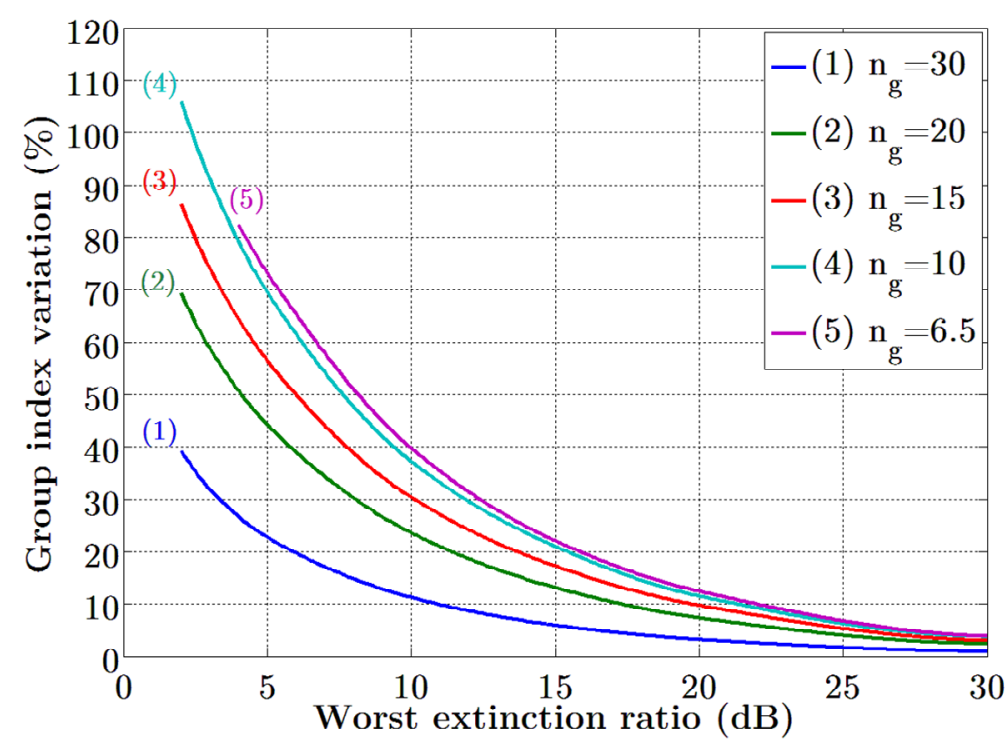

Figure 3-39: Tolerance on the group index variation (in \%) versus the worst extinction ER.

The optical bandwidth may also be defined as a function of the ER "ontarget" for a fixed group index. Since the notch has a finite width and becomes narrower for lower power, the optical bandwidth decreases as the ER "on-target" increases. Namely, for a design group index of 10, the bandwidths corresponding to ERs of $3 \mathrm{~dB}, 5 \mathrm{~dB}, 10 \mathrm{~dB}, 15 \mathrm{~dB}, 20 \mathrm{~dB}, 30 \mathrm{~dB}$ are respectively $9.107 \mathrm{~nm}, 4.481 \mathrm{~nm}$, $1.716 \mathrm{~nm}, 0.889 \mathrm{~nm}, 0.4774 \mathrm{~nm}$, and $0.157 \mathrm{~nm}$ as depicted in Figure 3-40. 
(a)

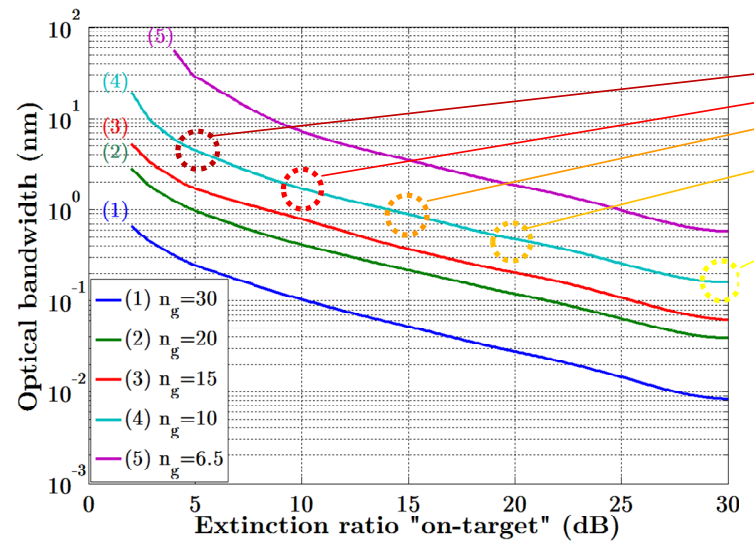

(b)

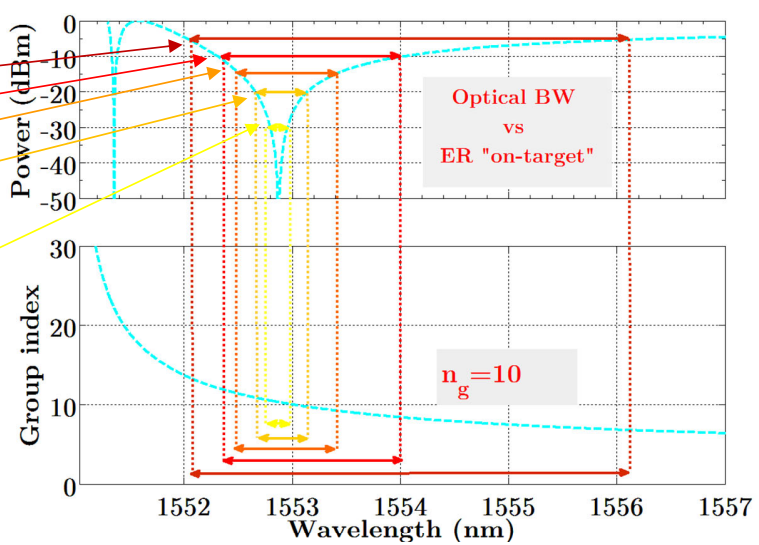

Figure 3-40: Optical bandwidth versus Extinction ratio "on-target"

(c) Temperature sensitivity: from a practical point of view, the group index variation with respect to the design value may be caused by external factors, such as temperature variations. Therefore, we provide here an estimate on the influence of temperature on the SW EOM performance. It is reminded that the SW EOM consists of a MZI with identical SWPS in both arms as depicted in Figure 3-38.

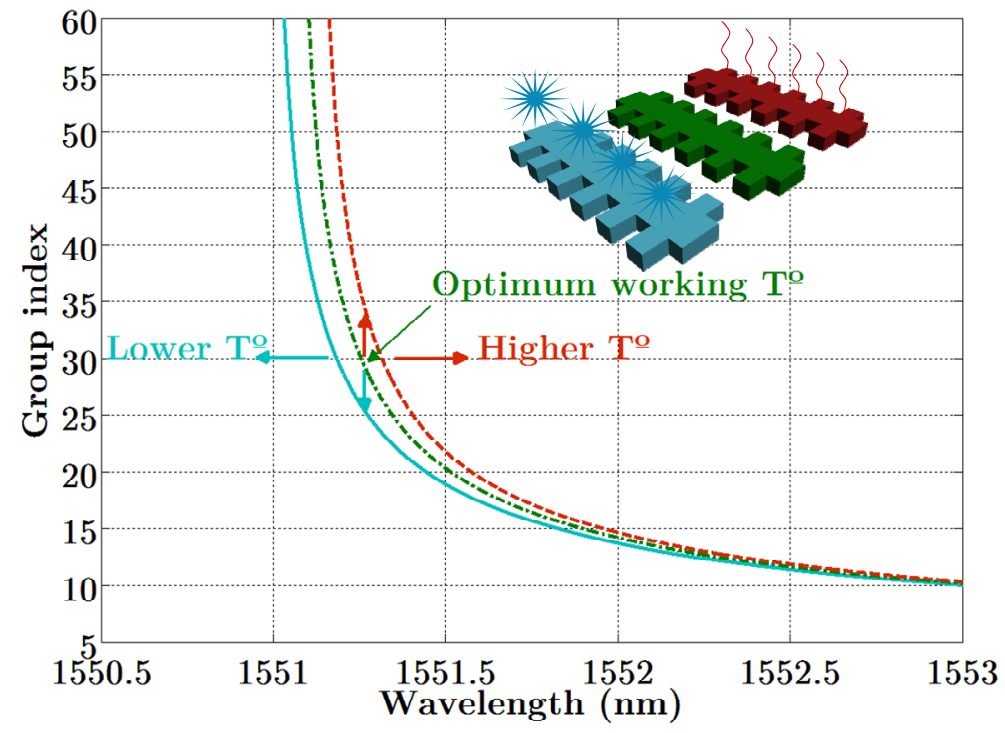

Figure 3-41: Influence of the temperature variation on the group index wavelength dependence

Therefore, the losses are assumed to be balanced when no voltage is applied. Furthermore, for the sake of simplicity, the produced refractive index change is considered to be uniform over the entire device. The temperature variations range 
from $-10^{\circ} \mathrm{C}$ to $+10^{\circ} \mathrm{C}, 0^{\circ} \mathrm{C}$ variations being the optimum working temperature. Positive (resp. negative) temperature variations causes the group index to increase (resp. decrease) as shown in Figure 3-41. Figure 3-42 shows to what extent the SW EOM performance in terms of DC ER "on-target" is affected by temperature variations. As expected, the tolerance to temperature variation is lower for higher group indices due to the steep group index dependency upon wavelength. Note that the remaining ER peaks for $n_{g}=15$ and 20 are due to overmodulation. In other words, for a fixed wavelength, the group index increases to approximately twice its design value as the temperature does, leading to a phase shift twice higher (i.e. a $2 \pi$ phase shift is achieved instead of $\pi$ ).

Table 3-7 summarize the temperature and wavelength spans as a function of the DC ERs "on-target", which clearly sets the allowable temperature variations on chip. This restriction becomes more severe as the group index increases. For instance, if the DC ER "on target" is $15 \mathrm{~dB}$, the allowable temperature range drastically drops from $12{ }^{\circ} \mathrm{C}$ to $1.4^{\circ} \mathrm{C}$ considering design group indices $n_{g}=10$ and $n_{g}=15$, respectively.

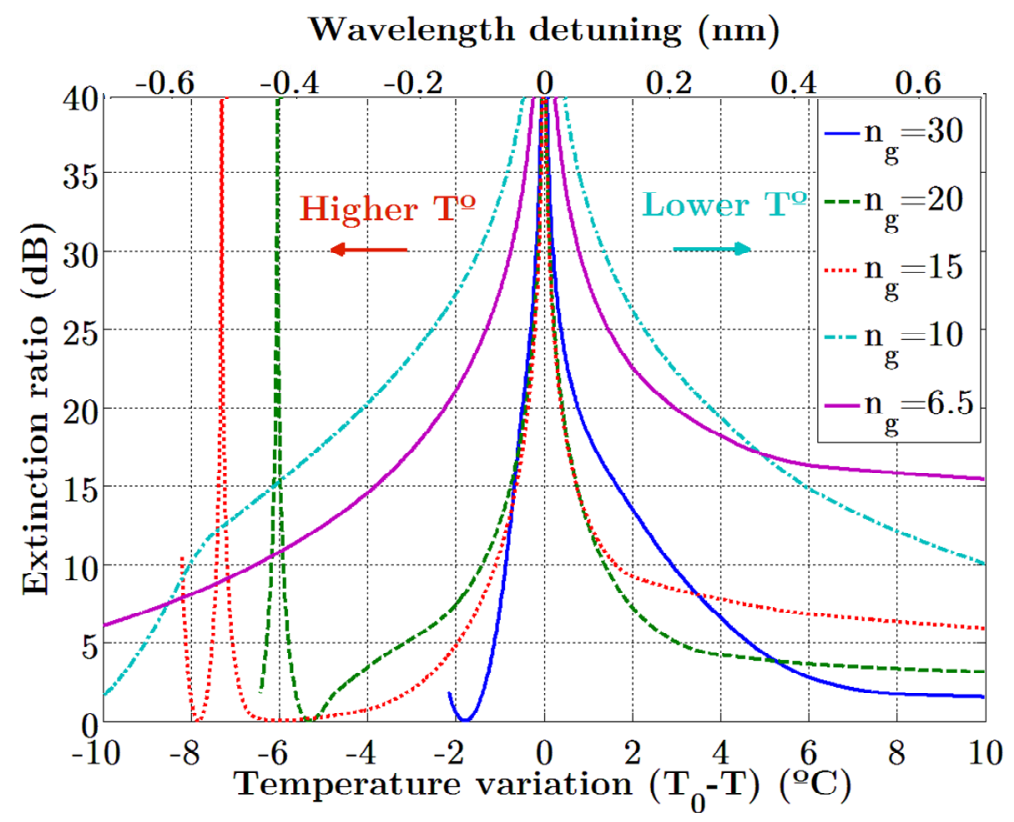

Figure 3-42: Influence of the temperature variation on the SW EOM extinction ratio for varying group index. The corresponding wavelength detuning is also shown on the abscissa. 


\begin{tabular}{|c|c|c|}
\hline $\operatorname{ER}(\mathrm{dB}) \mathbf{( n g = 6 , 5 )}$ & $\mathrm{T}^{\mathrm{o}} \operatorname{span}\left({ }^{\circ} \mathrm{C}\right)$ & $\boldsymbol{\lambda} \operatorname{span}(\mathrm{nm})$ \\
\hline 3 & $>20$ & $>20$ \\
\hline 5 & $>20$ & $>20$ \\
\hline 10 & $>15$ & 5,35 \\
\hline 15 & 13,8 & 1,13 \\
\hline 20 & 5,2 & 0,36 \\
\hline 30 & 1,5 & 0,11 \\
\hline
\end{tabular}

\begin{tabular}{|c|c|c|}
\hline ER $(\mathrm{dB}) \mathbf{( n g = 1 0 )}$ & $\mathrm{T}^{\mathrm{o}} \operatorname{span}\left({ }^{\mathrm{o}} \mathrm{C}\right)$ & $\boldsymbol{\lambda} \operatorname{span}(\mathrm{nm})$ \\
\hline 3 & $>20$ & 9,3 \\
\hline 5 & $>20$ & 1,97 \\
\hline 10 & 18 & 1,26 \\
\hline 15 & 12 & 0,85 \\
\hline 20 & 7,9 & 0,55 \\
\hline 30 & 2,8 & 0,2 \\
\hline
\end{tabular}

\begin{tabular}{|c|c|c|}
\hline ER $(\mathrm{dB}) \mathbf{( n g = 1 5 )}$ & $\mathrm{T}^{\mathrm{O}} \operatorname{span}\left({ }^{\mathrm{o}} \mathrm{C}\right)$ & $\boldsymbol{\lambda} \operatorname{span}(\mathrm{nm})$ \\
\hline 3 & $>12$ & 1,69 \\
\hline 5 & $>12$ & 1,09 \\
\hline 10 & 2,7 & 0,2 \\
\hline 15 & 1,4 & 0,1 \\
\hline 20 & 0,8 & 0,05 \\
\hline 30 & 0,3 & 0,02 \\
\hline
\end{tabular}

\begin{tabular}{|c|c|c|}
\hline ER $(\mathrm{dB}) \mathbf{( n g = 2 0 )}$ & $\mathrm{T}^{\mathrm{o}} \operatorname{span}\left({ }^{\mathrm{o}} \mathrm{C}\right)$ & $\boldsymbol{\lambda} \operatorname{span}(\mathrm{nm})$ \\
\hline 3 & 14,1 & 0,52 \\
\hline 5 & 6,1 & 0,44 \\
\hline 10 & 2,8 & 0,2 \\
\hline 15 & 1,5 & 0,1 \\
\hline 20 & 0,8 & 0,05 \\
\hline 30 & 0,3 & 0,02 \\
\hline
\end{tabular}

\begin{tabular}{|c|c|c|}
\hline ER $(\mathrm{dB})(\mathbf{n g}=\mathbf{3 0})$ & $\mathrm{T}^{\mathrm{o}} \operatorname{span}\left({ }^{\mathrm{o}} \mathrm{C}\right)$ & $\boldsymbol{\lambda} \operatorname{span}(\mathrm{nm})$ \\
\hline 3 & 7,1 & 0,5 \\
\hline 5 & 6,9 & 0,4 \\
\hline 10 & 3,1 & 0,26 \\
\hline 15 & 2,3 & 0,17 \\
\hline 20 & 1,3 & 0,58 \\
\hline 30 & 0,4 & 0,03 \\
\hline
\end{tabular}

Table 3-7: Tolerable temperature span and associated wavelength detuning for varying group index

Thermal management is of key importance when dealing with slow wave structures and more generally with integrated silicon photonic devices. This is especially relevant when we think of the perspective of integrating high speed electronic devices close to photonic components. Advanced electronic microprocessors may dissipate around $100 \mathrm{~W}$ of heating power which may lead to case temperature of around $60^{\circ} \mathrm{C}$ at the geometrical center of the device [117]. In addition, to maintain its level of performance and above all to prevent irreversible damages, the maximum fan inlet temperature should not exceed $35^{\circ} \mathrm{C}$ to $40^{\circ} \mathrm{C}$ depending on the processor model. Therefore, although a full thermal management study is beyond the scope of this thesis, it is clear that integrating high speed electronics close to SW EOMs and photonics devices in general, may affect their performance due potential thermal cross-talk. To overcome such a 
concern, one of the world's leading company in silicon photonics Luxtera has demonstrated that a simple and efficient heat sink or micro-heaters may be designed and integrated along with electronic drivers to maintain the performance of temperature sensitive devices[118] such as ring resonators. However if heat sinks and micro- heaters appears to be bulky and inappropriate in terms of size and/or power consumption, proper choice of layout parameters may also be an alternative solution to minimize or even avoid thermal crosstalk between electronic and photonic components. Other solutions, such as using overcoupled ring resonators in an unbalanced MZI with waveguides of different width [119] as well as using polymer coating (although it requires post processing) with negative thermo optic coefficient in order create athermal devices have been proposed[120]

To conclude, thermal management of integrated silicon devices is a key and relatively new topic in the field of silicon photonics, and packaging solutions will entirely depend on the overall design of each photonic or electro-photonic chip.

\subsubsection{Slow light propagation and 3dB roll-off bandwidth limitations}

Modulation speed is indeed a key aspect to evaluate the phase shifter performance. Such a bandwidth limitation may partly be due to the group velocity mismatch between the electrical and optical signals co-propagating respectively along the CPWG microwave line and the optical waveguide. This mismatch can be compensated by a convenient travelling wave (TW) design, enabling both waves to co-propagate with the same group velocities, i.e. when $n_{g, o p t}=n_{g, e l}$. Considering an open circuit configuration (no terminating resistances) [121], the limiting modulation frequency is given by :

$$
f_{3 d B}=\frac{0.556 \cdot c}{L_{\pi} \cdot n_{g, o p t}} \frac{1}{\left|1+\frac{n_{g, e l}}{n_{g, o p t}}\right|}
$$

Where $L_{\pi}$ is the interaction length, i.e. the required length to achieve a $\pi$-phase shift and $c$ is the speed of light in a vacuum. Assuming perfect matching between the group velocities of the electrical and optical signals, (i.e. $n_{g, o p t}=n_{g, e l}$ ) Equation (4.17) can be reduced to : 


$$
f_{3 d B}=\frac{0.556 \cdot c}{2 \cdot L_{\pi} \cdot n_{g, o p t}}
$$

For average group index values, say $n_{g, o p t}-3.7$, which were estimated from a 400x220nm rib silicon waveguide, higher 3dB roll-off bandwidths may be achieved for decreasing interaction length. As an example, under perfect velocity matching conditions (ideal case) and assuming a $3 \mathrm{~mm}$ interaction length, as typically required for carrier depletion-based MZI modulators, it follows $f_{3 d B} \sim 7.5 \mathrm{GHz}$. This means that open circuit configurations or lumped electrode configurations require short interaction lengths to avoid severe limitations on the modulation speed.

If we now assume that the electrical wave travels much faster than the optical wave $\left(n_{g, o p t}>>n_{g, e l}\right)$ as may happen in the case of slow wave modulators, Equation (4.17) can be approximately reduced to :

$$
f_{3 d B} \sim \frac{0.556 \cdot c}{L_{\pi} \cdot n_{g, o p t}}
$$

Assuming a group index $n_{g, \text { opt }} \sim 30$ and an interaction length of $\sim 400 \mu \mathrm{m}$, (see Figure 3 -27), it follows $f_{3 d B} \sim 13.8 \mathrm{GHz}$. This relatively low value arises essentially from fact that under velocity mismatching condition and open circuit configuration, increasing the group index value counteracts the achieved interaction length reduction and limits the $3 \mathrm{db}$ roll-off bandwidth.

However, if a careful TW electrode design including a terminating impedance is made (closed circuit configuration), the $3 \mathrm{~dB}$ roll-off frequency becomes:

$$
f_{3 d B}=\frac{0.556 \cdot c}{L_{\pi} \cdot n_{g, o p t}} \frac{1}{\left|1-\frac{n_{g, e l}}{n_{g, o p t}}\right|}
$$

The TW design is made such that $n_{g, o p t}=n_{g, e l}$, leading to an infinite $3 \mathrm{~dB}$ roll-off bandwidth. In practice however, it is quite a challenge to achieve perfect matching between the velocities of the electrical and optical signals owing to the possible experimental deviations on the microwave and optical group index values. Therefore, in the case of slow wave modulators one should take special 
care in designing the microwave transmission line as well as selecting operating points with moderate optical group index.

\subsection{Evolution of the Slow Wave Modulator Electrical Design}

The previous modeling was carried out with the aim of showing that on one hand SW EOMs are theoretically feasible and on the other hand that we could expect strong improvements in terms of performance at the expense of some potential drawbacks. The early design of our SW EOM consisted basically in a LCWG embedded into an asymmetric pn junction [122]. The electrical design was initially proposed in [114] and soon after demonstrated in an low footprint silicon ring resonator [35]. However, as previously mentioned asymmetric pn junction require precise alignment between the waveguide and the hard mask during the ion implantation step. In a CMOS process, alignment errors may be on the order of $\pm 100 \mathrm{~nm}$, which is relatively high compared to 400 to $500 \mathrm{~nm}$ wide waveguides. The positional deviation of the $p n$ junction associated with dopant activation issues [35] may results in a severe decrease of the expected efficiency of the modulator. These reliability issues might well be unacceptable for mass production purposes.

To overcome such reliability problems and improve the modulator yield, a widely employed fabrication process known as "self aligned fabrication process" in the CMOS industry has been introduced recently in $220 \mathrm{~nm}$ thick silicon EOM prototyping [49]. This has been considered of critical importance for HELIOS [123] project objectives. Briefly, HELIOS is an integrated project funded by the European Union, whose objective is to combine photonic layers with a CMOS circuit, using microelectronics fabrication processes and to make this technology available to fabless research institutes and companies. Basically, HELIOS aims at demonstrating complex functions, addressing a variety of industrial needs such as:

- a $40 \mathrm{~Gb} / \mathrm{s}$ modulator on an electronic IC

- a 16x10 Gb/s transceiver for WDM-PON applications,

- a Photonic QAM-10Gb/s wireless transmission system

- a mixed analog and digital transceiver module for multifunction antennas. 
SW EOMs based on the self aligned fabrication process is also part of HELIOS objectives. Consequently, since the first theoretical proposal [122] based on an asymmetric pn junction, the electrical design of the SW EOM based on LCWGs has evolved towards the reliability and performance of the "self aligned fabrication process" as pictured below (Figure 3-43).

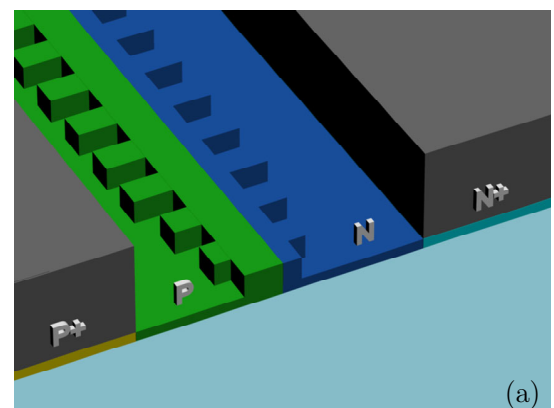

Assymetric PN junction

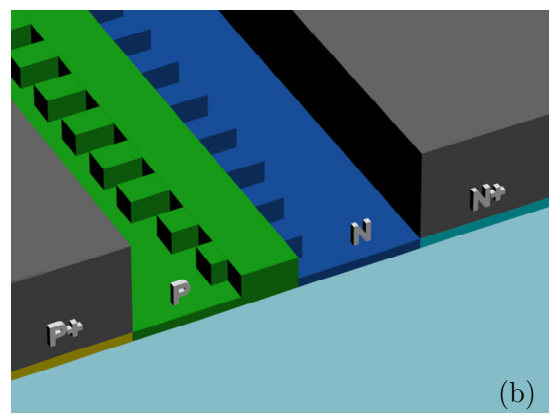

Self aligned PN junction

Figure 3-43: Slow wave modulator electrical design evolution. From asymmetric to self aligned $p n$ junction

\subsection{Fabrication Process}

\subsubsection{Technology overview}

Today's trend in silicon photonics is demonstrating that any new device design may be mass produced in CMOS foundries. Such entities consist of advanced nanofabrication tools and highly experienced engineers. Working in a controlled environment gives the designer the opportunity to be aware of the reality of fabrication constraints and capabilities. This positive feedback is of key importance in the emerging field of silicon photonics because it opens the way to relevant prototyping for industrial interests.

The complexity of our SW EOM and more generally active silicon devices requires numerous demanding fabrication steps, which must be handled cautiously to prevent time and money consuming errors. For the abovementioned reasons, a CMOS foundry provides a suitable platform to realize complex active silicon devices such as our SW EOM. In Europe, the foundry model relies on ePIXfab [101] within the FP7 framework, which organizes wafer-scale fabrication services of silicon photonic integrated circuits. It consists of two institutions: CEA-LETI [102] and IMEC [103], with similar standard fabrication capabilities summarized below. Design rules and guidelines are provided in the technology paper standard of both CEA-LETI [124] and IMEC [125]. 


\begin{tabular}{|c|c|}
\hline Wafer size \& type & $200 \mathrm{~mm}$ SOI \\
\hline Si layer thickness & $220 \mathrm{~nm}$ \\
\hline BOX thickness & $2 \mu \mathrm{m}$ \\
\hline DUV lithography resolution & $193 \mathrm{~nm} / 248 \mathrm{~nm}$ \\
\hline WG etch/ Fiber coupler etch & $220 \mathrm{~nm} / 70 \mathrm{~nm}$ \\
\hline
\end{tabular}

Table 3-8: Epixfab passive technology standards

In addition to passive technology, ePIXfab offers ion implantation services to realize silicon active devices based on the plasma dispersion effect. The most common impurities used to dope silicon are boron and phosphorus, which are respectively situated in the $3^{\text {rd }}$ column and $5^{\text {th }}$ column of the periodic table. Finally, silicide and metallization are also available processes to form low loss contacting electrodes.

\subsubsection{Process flow description}

Realizing SW EOMs requires a series of highly controlled fabrication steps summarized below. As depicted in Figure 3-44 (a), the process starts with light Ptype implantation, through positive resist window opening in a $220 \mathrm{~nm}$ thick silicon layer on top of a $2 \mu \mathrm{m}$ thick buried oxide layer (BOX). This forms the Ptype background. (b) Then, the resist is removed (stripping) and a $\mathrm{SiO} 2$ layer is deposited. (c) Next, a lithographic step followed by an etching is performed to define the waveguide $\mathrm{SiO}_{2}$ hard mask. (d) The $220 \mathrm{~nm}$ thick silicon layer is then partially etched down to $100 \mathrm{~nm}$ or $50 \mathrm{~nm}$ depending on the electrical and LCWG design. (e) The $\mathrm{SiO}_{2}$ hard mask is left on top of the waveguide and the edge of the resist windows may be approximately positioned anywhere over the latter. (f) Therefore, the $\mathrm{SiO}_{2}$ mask in combination with the resist layer window opening enables the positioning of the left side of $n$-type implantation area just at the edge of the waveguide. In other words, the junction is self aligned with the waveguide edge [49]. (f) Then, $p^{+}$type and $n^{+}$type implantation are carried out separately through lithography and hard mask window opening. Dopant electrical activation is performed through Rapid Thermal Annealing (RTA). (g) Finally, the device is covered with a $\mathrm{SiO}_{2}$ upper cladding, windows are opened on the $\mathrm{p}_{+}$and $\mathrm{n}_{+}$type region. A metal layer is deposited on top and etched to form ohmic contacting 
electrodes. Including the intermediate physical characterizations, there are approximately 35 fabrication steps.

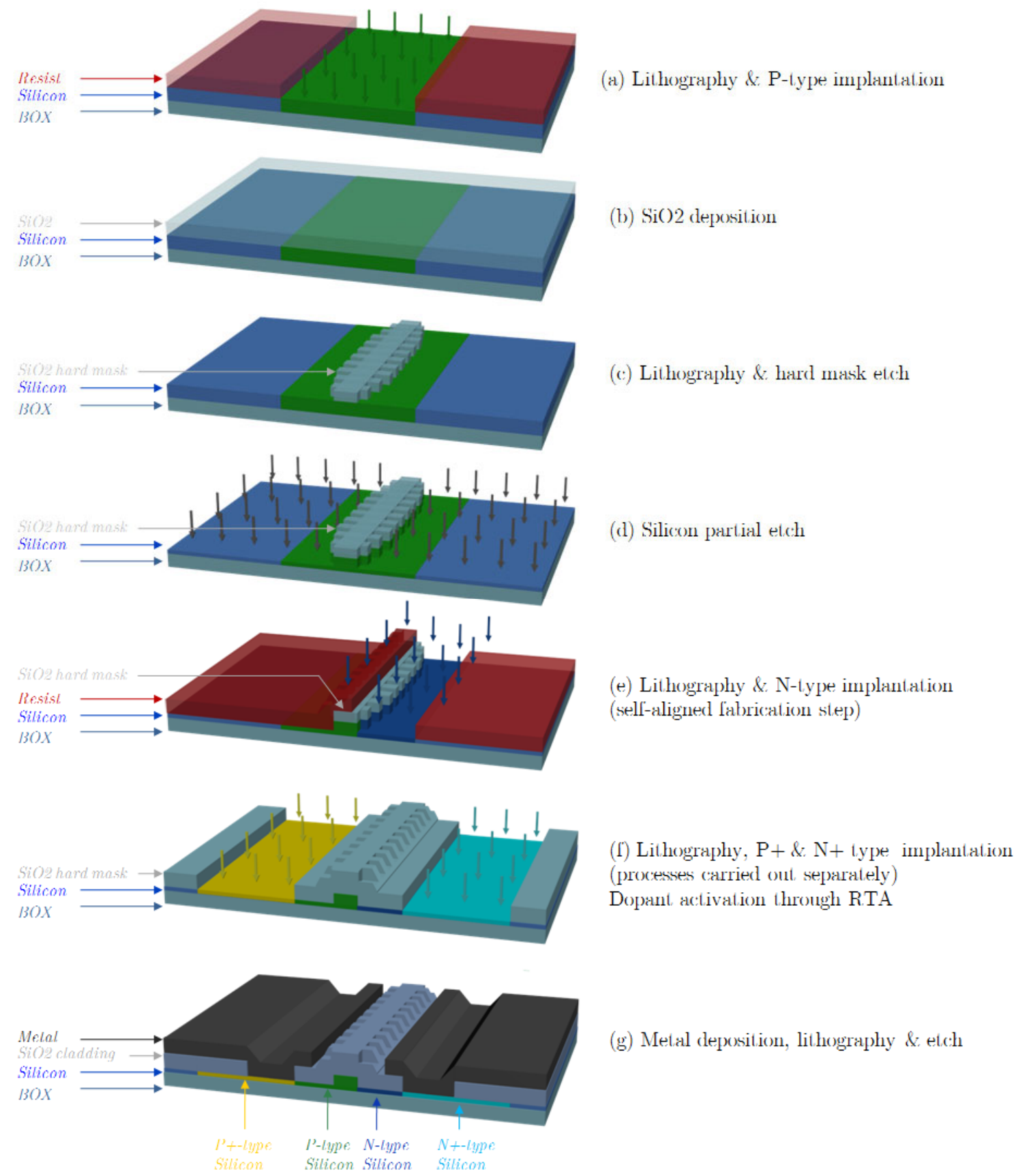

Figure 3-44: Slow wave EOM fabrication process (lithography and stripping intermediate steps are not represented for simplicity) developed by Thomson et al. [49]

In conclusion, based on the available CMOS technology provided by the two major European silicon photonic foundries, we have presented an overview of the fabrication process of our SW EOM. The reason for choosing CMOS 
industrial platform is twofold: Achieving high performance devices to create a significant scientific impact in the field of silicon photonics and demonstrating that these results can reliably be reproduced in an industrial environment. To support such objectives, the next section is devoted to our experimental results.

\subsection{Experimental Results}

\subsubsection{Passive characterization overview}

Prior to demonstrating enhanced high speed modulation using the slow wave effect, we proceeded to the passive characterization of the periodic structures. The first step was to show that the slow wave effect could reliably be achieved in silicon LCWGs. For such a purpose, two runs of passive structures were carried out on a CMOS platform. The first run included only "brut force" designed passive structures to acquire some experimental background. The second run consisted of both optimized passive structures as well active devices including doped LCWGs for the demonstration of EO modulation under a slow light enhancing scheme.

\subsubsection{Passive characterization results}

\section{(a) Run 1 (preliminary design):}

\section{Straight LCWGs characterization}

The first batch contains slow wave structures with $W=300 \mathrm{~nm} \times H=220 \mathrm{~nm}$ rib photonic wire Bragg grating partially etched to leave a $50 \mathrm{~nm}$ slab as depicted in Figure 3-45. The designed "teeth" parameters are $W e=550 \mathrm{~nm}$ and $W i=150 \mathrm{~nm}$, which are periodically repeated over a period $a=330 \mathrm{~nm}$. The fabrication was carried out at CEA-LETI on 8 inches wafer provided by SOITEC, with $193 \mathrm{~nm}$ Deep UV lithographic process. 
(a)

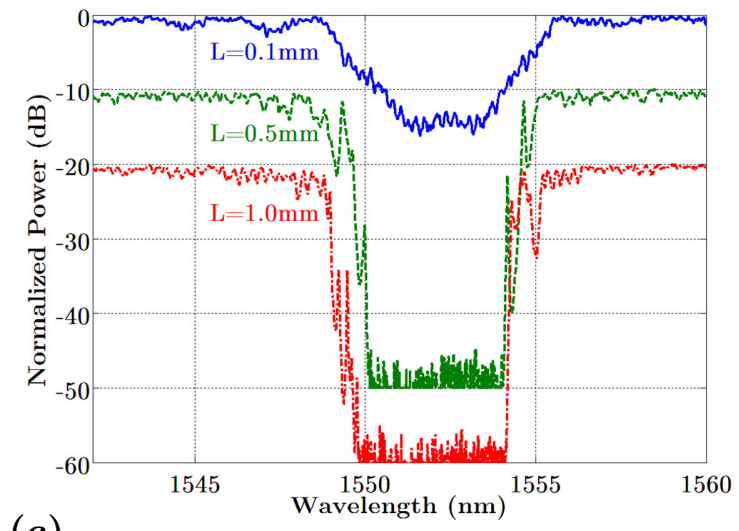

(c)

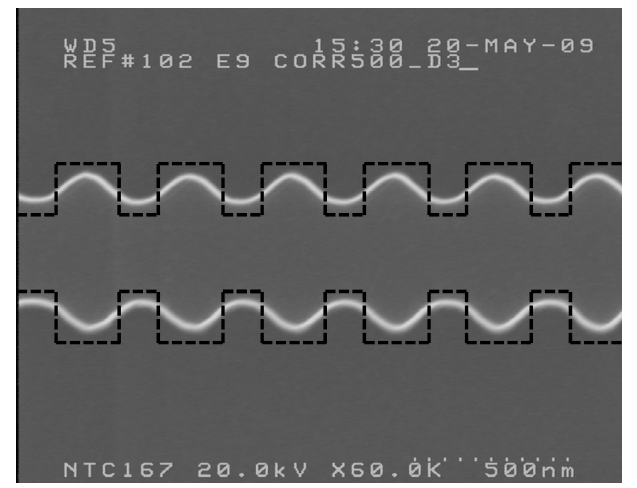

(b)

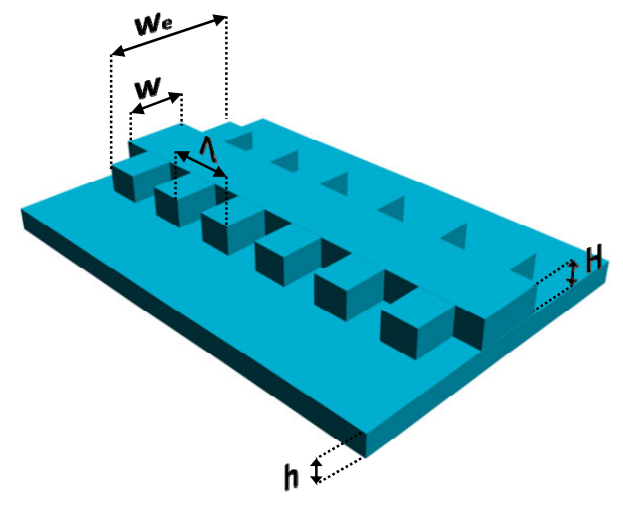

(d)

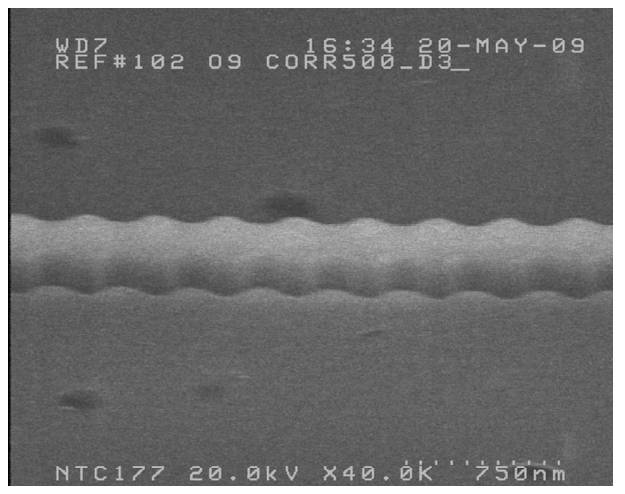

Figure 3-45: (a) Transmission spectra of the $0.2 \mathrm{~mm}, 0.5 \mathrm{~mm}$ and $1 \mathrm{~mm}$ long LCWGs. Normalized spectra are shifted vertically by $10 \mathrm{~dB}$ for clarity (b) Waveguide overview (c), (d) SEM images of the actual fabricated LCWGs. The dashed line is the waveguide contour as it appears on the mask.

The measured spectra depicted in Figure 3-45 (a) show the strong dependence of the photonic band gap extinction ratio upon the grating length. The $100 \mu \mathrm{m}$ long photonic wire Bragg grating exhibits a $5 \mathrm{~nm}$ wide gap with a 15 dB extinction ratio. Both 500 and $1000 \mu \mathrm{m}$ long corrugated waveguides presents very similar characteristics, featuring however much deeper band gaps of about 40 $\mathrm{dB}$, due to their higher reflectivity. Despite a clear deviation in shape (the actual corrugations appear to be rounded off) of the LCWGs due to the difficulty to resolve the small grating teeths (see 3.3.3 (a)), the periodicity is maintained. This means that slow wave propagation may still be achieved. As seen, the slow wave regions, i.e. $\sim 2 \mathrm{~nm}$ away from both sides of the photonic band gap edges, are localized around $1.55 \mu \mathrm{m}$. The ripples close to these edges are typically due to the fast to slow mode impedance mismatch at the rib to corrugated waveguide transition. This is because both ends of the LCWG are highly reflective and hence form a Fabry-Pérot (FP) cavity. This high reflectivity is due to the absence of efficient fast to slow light coupling via optimized tapers. 


\section{Group index determination}

The LCWGs have been inserted in two 0.25 an $0.5 \mathrm{~mm}$ long MZIs in order to estimate the achieved group index values via an "on-chip" interferometric method" as depicted in Figure 3-46. The MZI consists of two arms of identical length, one having a LCWG and the other having a $300 \mathrm{~nm}$ wide rib waveguide to match the width of the narrow section of the LCWG. The group index has been calculated from the following formula:

$$
n_{g}(\lambda)=\frac{\lambda_{\min } \lambda_{\max }}{2 L \Delta \lambda}+n_{g, r e f}
$$

Where $\lambda_{\min }$ and $\lambda_{\max }$ are the wavelength corresponding to the extrema of the MZI fringes, $L$ is the LCWG length and $n_{g, r e f}$ is the group index of the reference rib waveguide. From Figure 3-46 (a), we may notice that the interference fringes exhibit ripples. These are due to the non-optimized fast to slow light coupling caused by FP interference and hence power variations inside the cavity formed by the LCWG. Therefore, to provide a measure of confidence in the group index determination, error bars are also added to each extracted group index value as depicted in Figure 3-46 (b) (c). Following the reasoning of Vlasov et al [70] the interval of confidence is estimated from the maximum value of uncertainty in assigning the position of the extrema. In other words, the error bars cover the range of group index values that would be extracted considering the possible error in determining the extrema position caused by the (FP) noise.

As seen on both sides of the spectra, the propagating light is significantly slowed down although it seems that the maximum achieved group index values are strongly dependent on the considered slow mode. Indeed, the fundamental mode of the $0.25 \mathrm{~mm}$ long LCWG exhibits a group index value in excess of $\sim 55$ at $1.555 \mu \mathrm{m}$ wavelength while the first order mode features maximum group index of only $\sim 17$. Likewise the respective maximum group index values of the fundamental and first order mode of $0.5 \mathrm{~mm}$ long LCWG are around $\sim 9$ and $\sim 21$. A simple explanation might be that the first order slow mode is lossier than the fundamental one. The difference is not really significant under fast light propagation, but becomes noticeable in the slow light regime. In addition, and consistently with the effective index values, the mode profiles illustrated in the insets in Figure 3-46 (b) (c) show that for the fundamental mode (respectively first order mode), most of the field is confined within the wide section 
(respectively narrow section) of the LCWG, which makes it probably less sensitive to scattering and substrate leakage loss[126].

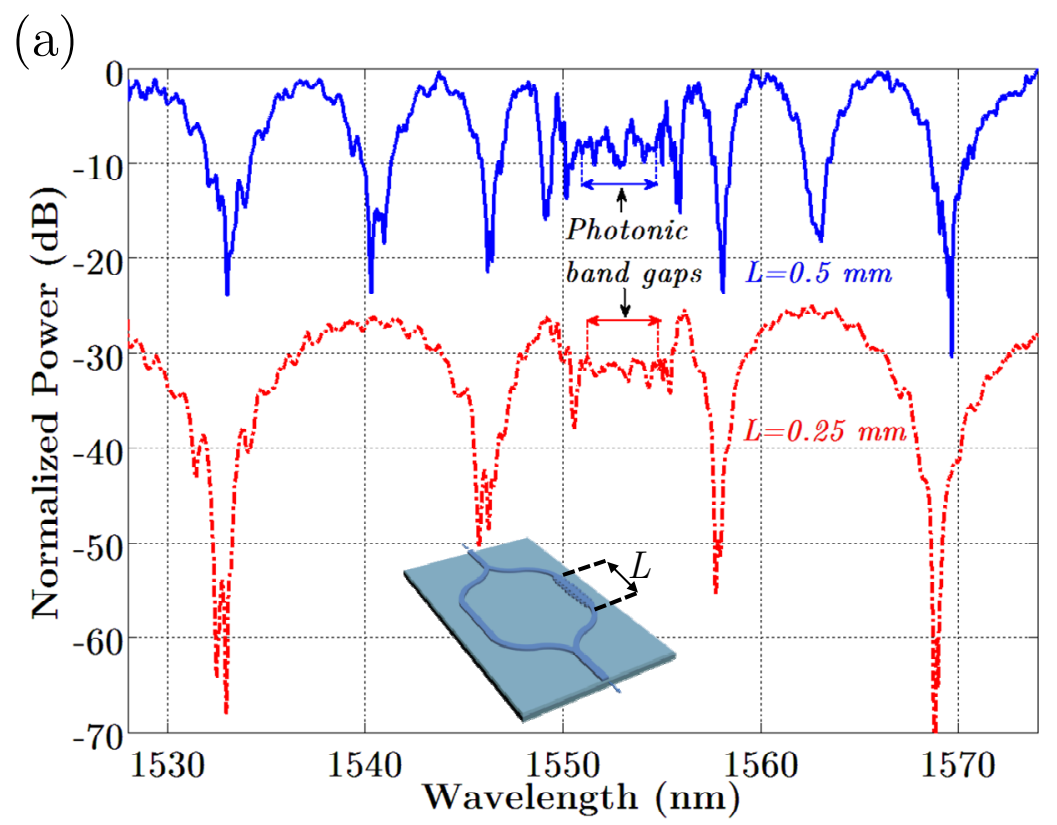

(b)
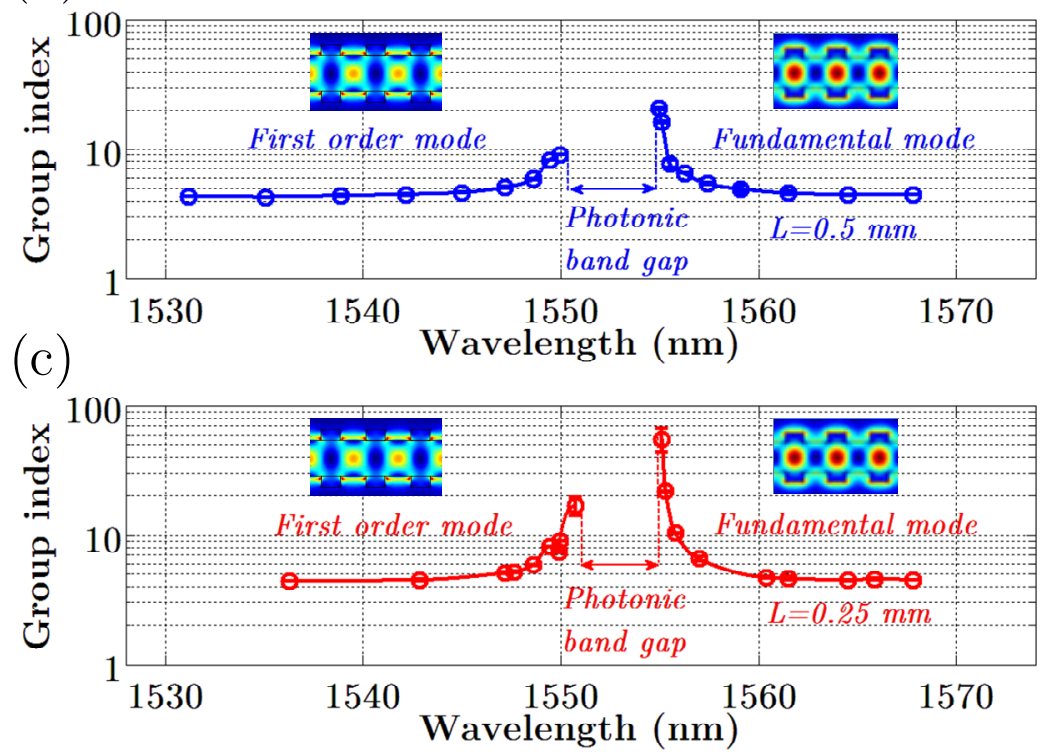

Figure 3-46: (a) transmission spectra of two MZI loaded with one LCWGs of respective length $L=0.5$ and $0.25 \mathrm{~mm}$ shown in the inset (b) and (c) Calculated group index versus wavelength for respective LCWG lengths of $L=0.5$ and $0.25 \mathrm{~mm}$.

This may be directly related to the fact that there are more interferences fringes within the fundamental slow mode region than in that of the first order slow mode. As a result, higher group index values may be extracted. Additionally, 
increasing the LCWG length by two rules out the extraction of higher group indices due to the higher net propagation losses experienced by both modes.

\section{Discussion}

From these preliminary passive results, we have pointed out several issues.

First, the mismatch between the designed and actual slow wave waveguides arises essentially from the fact that the corrugated elements are on the order of the wavelength deep UV exposure light, which leads to optical proximity effects. Therefore, a convenient design is expected to anticipate such a divergence. Second, the FP noise affects the fringe visibility, thus two input and output tapers should be designed to smoothen the optical transition from the fast to slow light regime and hence help decreasing at the same time the coupling losses.

Third, the optical path length between both arms of the MZI should be increased to augment the number of interference fringes within the slow wave region and thus the amount of modulation points for a group index dependent slow wave modulator study.

To summarize, the action points are therefore:

- Minimizing the corrugation rounding by compensating for optical proximity effects

- Decreasing the fast to slow light coupling losses by adding tapers at the rib to LCWG transitions

- Increasing the optical path length between arms to provide more modulation points.

\section{(a) Run 2 (optimized designs):}

To address the abovementioned action points, a second run including the required modifications was realized.

\section{Tackling corrugation rounding}

To minimize optical proximity effects, the shape of the corrugations should be altered in advance on the layout if the minimum feature size is smaller than the wavelength of light used for the exposure (see 3.3.3 (a)). In other words, this is exactly what happens to the corrugated waveguide since it consists of closely packed elements, whose size and respective separation are on the order of the 
illuminating wavelength. Examples of the shapes that have been designed prior to the deep UV exposure to minimize the rounding problem are shown below.

As designed on the Mask

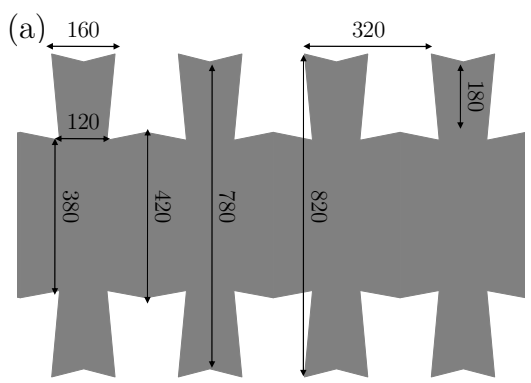

(b)

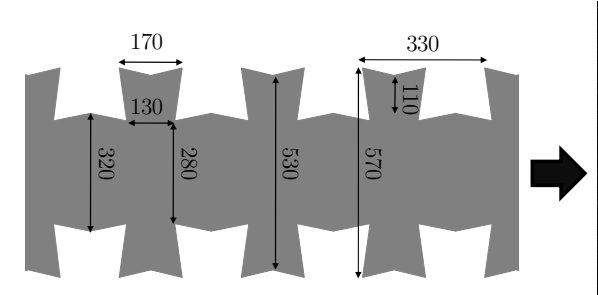

(d) Expected \& actual waveguide

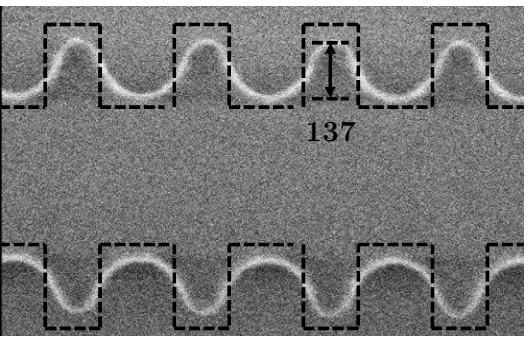

(e) (c)

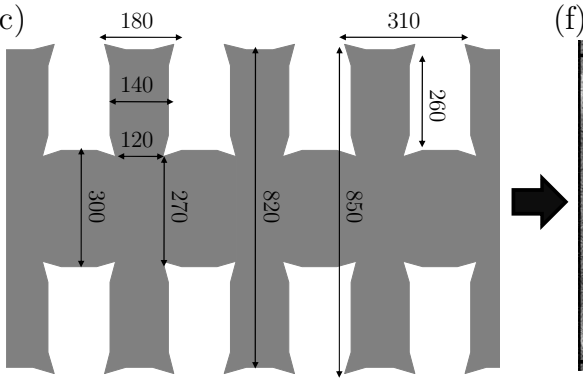

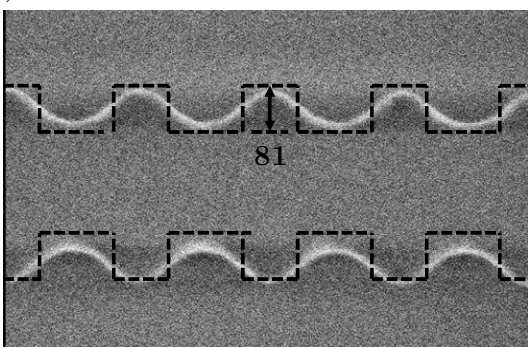

(f)

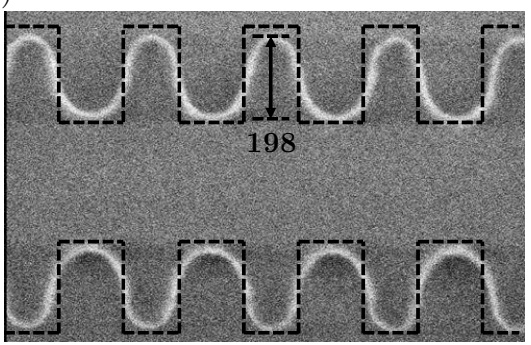

Figure 3-47: (a), (b) and (c) Corrugated waveguides as designed on the mask corresponding respectively to design 5, 6 and 7 (d), (e), (f) Top views of the expected (dashed line) and actual corrugated waveguides (SEM images).

\section{Coupling to the slow light mode}

Coupling to the slow light mode is also a commonly encountered issue that may lead to unwanted reflections at both input and output of any slow light waveguide. This is due to the fact that the waveguide acts as a cavity with two reflecting mirror which cause losses and FP ripples. However, a convenient taper design for both 2D line defect PhCs and LCWGs and may help minimizing this problem as already demonstrated in several works[110, 113]. Following the idea proposed in [110], the coupling may be enhanced via a gradual increase of the width of the corrugations. Although this idea is attractive, restrictions in terms of teeth width may arise due to the deep-UV limited resolution. Indeed, the minimum tolerated feature is $50 \mathrm{~nm}$. In other words, the number of tapered 
elements will be restricted, leading possibly to an imperfect impedance matching between the fast and slow light mode. To address such an issue, tapers with a limited number of elements have been designed as shown in Figure 3-48.

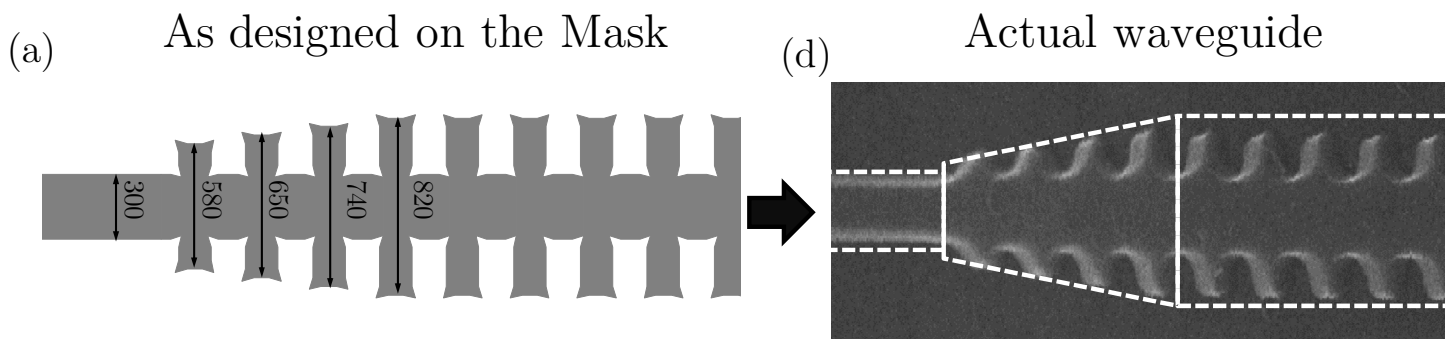

Figure 3-48: (a) Taper as designed on the mask (b) Actual taper

\section{Path length}

In order to provide enough modulation points for a comprehensive group index dependent study, the asymmetry, i.e. the optical path length of the MZI should be increased to reduce the FSR. For such a purpose, the optical path length difference between arms is set to $0.9 \mu \mathrm{m}$, leading to a theoretical FSR of $\sim 1.1 \mathrm{~nm}$ within the fast light region.

\section{Straight LCWGs characterization}

To localize the band gaps and the slow wave regions in straight LCWGs (here, only designs 6 and 7, corresponding to (a) and (c) of Figure 3-47 are considered) of length $0.2,0.5,1$ and $2 \mathrm{~mm}$ have been optically characterized. As expected from the theoretical simulations, a larger contrast between the narrow and wide sections of the LCWGs, leads to an increase in the photonic band gap width. This may be observed comparing the transmission spectra of design 6 (shallow corrugations) and 7 (deep corrugations) shown in Figure 3-49 below. The transmission ripples on both sides of the photonic band gap are due to the improved but still non-optimum coupling between the fast to slow light modes in spite of the tapering sections added a the waveguide extremities. The ripple wavelength decreases as we move towards the photonic band edge due the increasing group index. Moreover, the ripple wavelength also decreases for increasing cavity length formed by the LCWGs. 
(a)

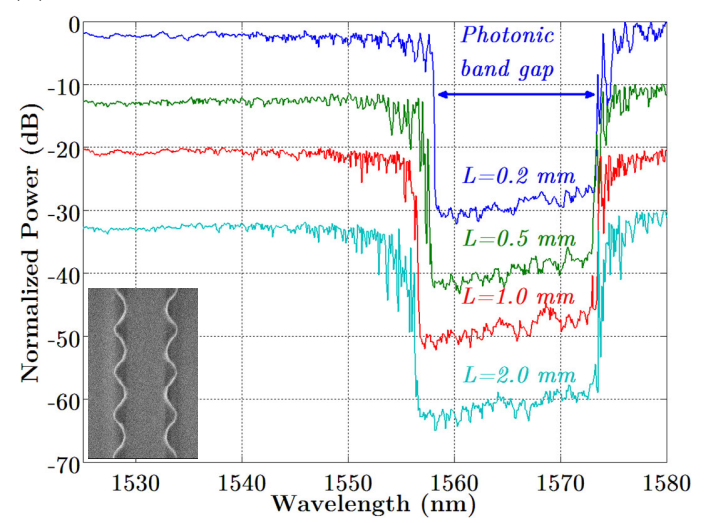

(b)

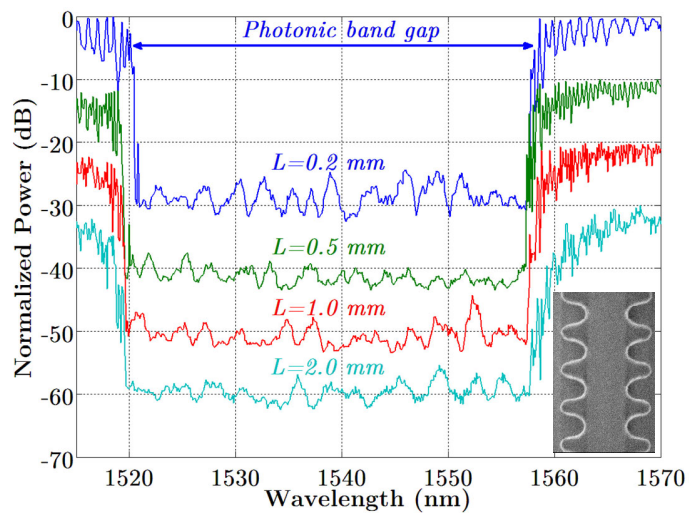

Figure 3-49: (a) resp. (b) Transmission spectra of the $0.2 \mathrm{~mm}, 0.5 \mathrm{~mm}, 1 \mathrm{~mm}$ and $2 \mathrm{~mm}$ long LCWGs corresponding to design 6 (shallow corrugations) and 7 (deep corrugations). Normalized spectra are shifted vertically by $10 \mathrm{~dB}$ for clarity. Insets are SEM images of the fabricated LCWGs.

\section{Group index determination in doped LCWGs}

Just as for the first set of LCWGs, the group index is determined via a direct interferometric method except that here the slow wave waveguides are embedded in the self aligned $p n$ junction to form the SWPS. It should be noticed that doping may significantly increases the losses due to free carrier absorption and crystalline defect generated by the implantation process. Slow wave propagation is expected to enlarge these loss mechanisms due to stronger light matter interaction, which may eventually lead to lower achieved group indices due to the altered fringe visibility. More details about the doping profile are given in the following section.

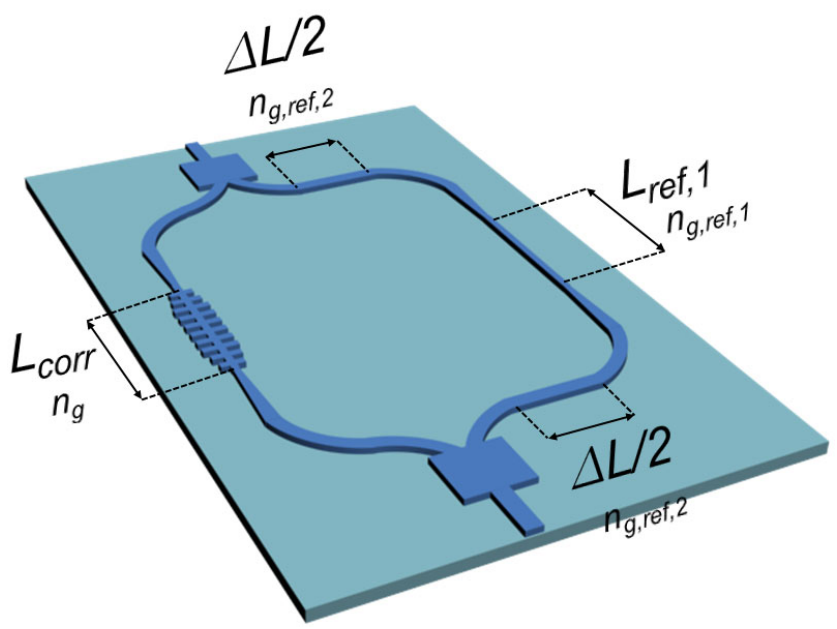

Figure 3-50: Overview of the slow wave MZI. The silicon slab is not depicted for clarity 
From Figure 3-50, we can see that the doped LCWG is placed in the shorter arm of the MZI while a conventional rib waveguide with the same length and doping conditions is placed in the longer arm as a reference. The width of the reference rib waveguide was reduced to match that of the narrow section of the slow wave corrugated waveguide.

Figure 3-51 and Figure 3-52 provide an overview of the spectra corresponding to the $1 \mathrm{~mm}$-long doped LCWG and MZI loaded with the same waveguide. As can be seen, the slow wave mode signatures are clearly visible at the photonic band edges from to the FSR variations across wavelength. Indeed, the FSR of the MZI's interference fringes in the normalized transmission spectra can be calculated as follows

$$
F S R \approx \frac{\lambda^{2}}{n_{g}(\lambda) \cdot L_{c o r r}-\left(n_{g, r e f, 1} \cdot L_{r e f, 1}+n_{g, r e f, 2} \cdot \Delta L\right)}
$$

where $\lambda$ is the central wavelength of the FSR, $n_{g}, n_{g}$, ref,, and $n_{g}$, ref, 2 are the respective group indices in the slow wave, $300 \mathrm{~nm}$ wide reference rib and $400 \mathrm{~nm}$ wide rib waveguides, $L_{\text {corr }}$ and $L_{r e f, 1}$ are the lengths of the slow wave and reference rib waveguides and $\Delta L$ stands for the length difference between the MZI arms considering only the $400 \mathrm{~nm}$ wide rib waveguide. For both MZI configurations, $L_{\text {corr }}=L_{r e f, 1}=1 \mathrm{~mm}, \Delta L=0.9 \mathrm{~mm}, n_{g, r e f, 1} \approx 3.5$ and $n_{g, r e f, 2} \approx 3.7$.

At wavelengths farther away from the photonic band gap edge, the group index of the slow wave waveguide is approximately constant with wavelength as in the rib waveguide. This originates from the fact that the backward and forward propagating waves no longer interact due to the lack phase matching leaving total internal reflection (TIR) as the dominant propagation mechanism. As a result, the FSR remains also constant. However, because the group index increases as we move closer to the band edge, the FSR initially increases. According to Equation(4.20), the FSR is infinite when the denominator is zero, which means that there is no phase difference between the arms and the MZI is optically balanced. It can be straightforwardly obtained that this occurs for a group index of $\sim 6.8$. Beyond this point, higher group indices in the slow wave waveguide cause the FSR to decrease, as depicted in Figure 3-51 and Figure 3-52. The FSR value calculated from Equation (4.20) becomes negative because the effective propagation length of the shorter arm turns out to be larger than that of 
the longer arm. While this may be clearly observed in the MZI transmission spectrum of design 7 (deep corrugations, see Figure 3-52), this becomes less obvious for design 6 (shallow corrugations, see Figure 3-51). Indeed, it seems that beyond this balanced point, the fringes attenuate in a stronger manner in design 6 than in design 7 . This may be explained by the fact that the optical bandwidth of the slow light region (approximately from $n_{g} \sim 5$ to $n_{g} \sim n_{g}$, max) is roughly twice larger for design 7 than for design 6 (7.8 $\mathrm{nm}$ versus 3.5nm, respectively). Indeed, PWE simulations suggests that the deeper the corrugations, the larger the band gap and eventually the smoother the increase in group index. In the case of design 6 , the limited FSR prevents from providing enough fringes to resolve the slow wave region.

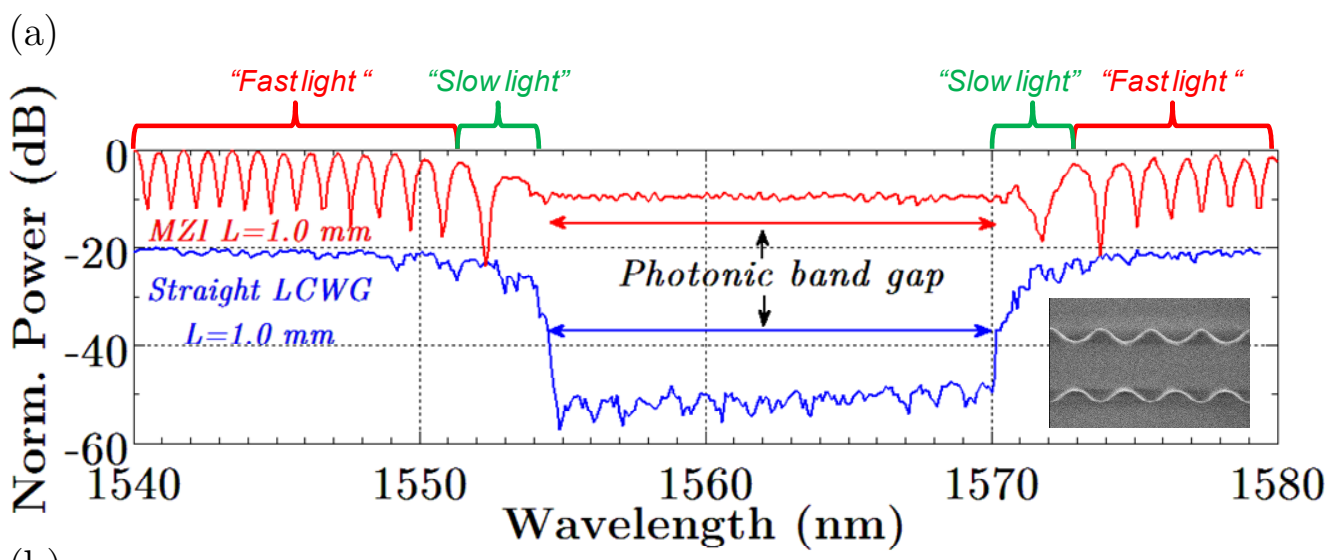

(b)

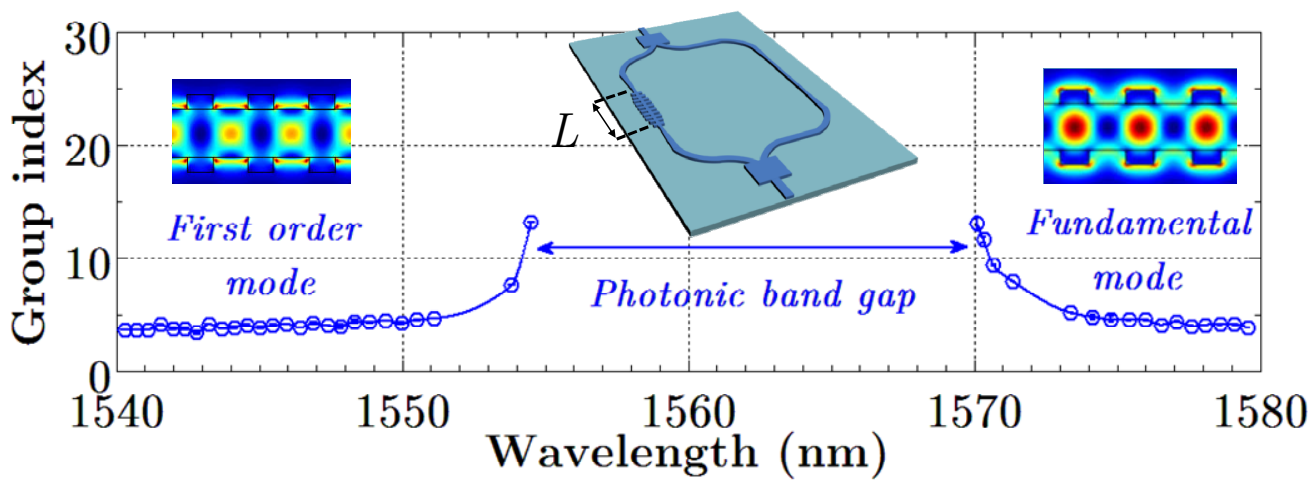

Figure 3-51: (a) transmission spectra of an assymetric MZI with one doped LCWGs of length $L=1 \mathrm{~mm}$ placed in the shorter arm. Normalized spectra are shifted vertically by $20 \mathrm{~dB}$ for clarity. (b) Calculated group index versus wavelength for a LCWG length of $L=1 \mathrm{~mm}$. The LCWG corresponds here to design 6 (see (a) and (b) of Figure 3-47). 

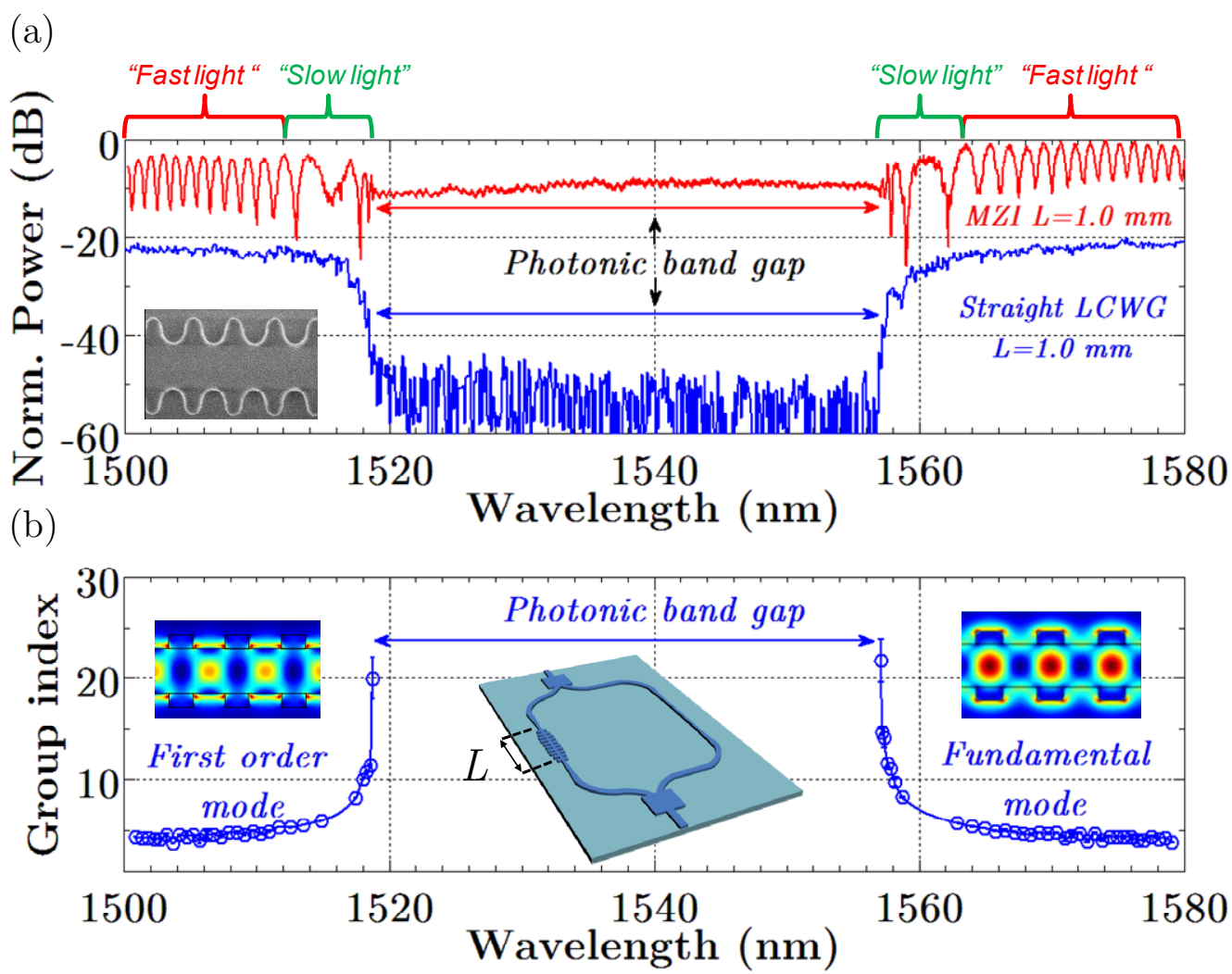

Figure 3-52: (a) transmission spectra of an assymetric MZI with one doped LCWGs of length $L=1 \mathrm{~mm}$ placed in the shorter arm. Normalized spectra are shifted vertically by $20 \mathrm{~dB}$ for clarity. (b) Calculated Group index versus wavelength for LCWG length of $L=1 \mathrm{~mm}$. The LCWG corresponds here to design 7 (see (c) and (f) of Figure 3-47).

An interesting feature of doped LCWGs is that their strong FP power oscillations at the band edges seem to vanish, which is likely due to the loss increase within the cavity. Additionally, one may notice that doping also blue shifts the LCWG transmission spectra of around $\sim 2$ to $4 \mathrm{~nm}$ as a result of the slight refractive index change, which may be imputable to the presence of impurities within the material. This has to be taken considered when redesigning the SW EOM. Our calculations show that group index values in excess of 13 (resp. 20) are readily achievable in doped LCWG design 6, (resp. Design 7). Because high group index values and good spectral characteristics such as deep and numerous notches in the slow wave region are highly desirable, we believe that design 7 is most suitable for a complete experimental study of the enhancement effect of slow light on electro-optical modulation. 


\section{Interval of confidence for group index measurements}

To provide a measure of confidence in the group index determination, error bars are also added to each extracted group index value. As expected the error grows significantly near to the photonic and gap due to the steep group index dependence upon wavelength. For completeness, error bars were also assigned to the calculated phase shift against voltage curves. The uncertainty on the phase shift calculation originates essentially from the FSR determination. As in the case of the group index, the error bars cover the maximum uncertainty on the FSR estimation for each applied voltage.

\subsubsection{Active characterization overview}

The testing method consists in evaluating first the device static (DC) performance, i.e. figure of merit, insertion losses, achieved phase shift and interaction length. Then, we will evaluate the high speed performance by sending electrical data through high speed probes at microwave frequencies. On the other hand, because slow light occurs in a certain range of wavelengths, and varies over the latter, the DC and high speed performance of the modulators will depend also upon the operating wavelength.

The strategy to be followed consists of three main points:

(a) Localizing the slow wave regions based on the passive characterization results

(b) Proceeding to DC tests and localizing the optimum operating point

(c) Proceeding to high speed tests

\subsubsection{Slow wave modulator overview}

Among the variety of SW EOM designs present on the wafer, we selected one in particular due to its suitable features for the demonstration of enhanced EO modulation in slow wave structures. As previously stated, the choice was made based on how well the MZI can resolve the slow wave region. In this case, "resolving" means providing as much notches as possible within the slow wave region to enable testing the slow wave modulator performance at different group index values. LCWGs with high effective index contrast (i.e. higher size difference between thin and wide sections) generally provide smoother group index 
dependence against wavelength as well as a wider band gap. As a result, the optical bandwidth is slightly increased. As previously mentioned, the smoother group index dependence of design 4 against wavelength makes it perfectly suitable for a group index dependent modulation study since the MZI spectra provides several notches and therefore as much modulation points.

Slow light propagation is achieved through the use of a LCWG, with narrow and wide sections of width $W=300 \mathrm{~nm}$ and $W_{e}=650 \mathrm{~nm}$, which are repeated over a period $a=310 \mathrm{~nm}$, as shown in Figure 3-53 (a), (b). The slow wave waveguide height is $H=220 \mathrm{~nm}$, which after partial dry etching process leaves a 100nm thick slab (designated $h$ in Figure 3-53 (c), (d)). Optical phase modulation is achieved by depleting the majority carriers from a reverse biased self aligned $p n$ junction [49] (see section 3.6.2) connected to highly doped $p^{+}$and $n^{+}$regions. These are situated respectively at a distance of $S_{n}=550 \mathrm{~nm}$ and $S_{p}=500 \mathrm{~nm}$ from the edge of the narrow waveguide section and covered with compound $\mathrm{AlCu}$ electrodes in order to ensure good ohmic contacts. Net doping concentrations in the $p$ - and $n$-type regions reached respectively $3.10^{17} \mathrm{at} / \mathrm{cm}^{-3}$ and $1.5 .10^{18} \mathrm{at} / \mathrm{cm}^{-3}$. Highly $p^{+}$and $n^{+}$doped regions were implanted both at a concentration of $1.10^{20}$ at $/ \mathrm{cm}^{-3}$. Figure 3-53 shows the schematic of the designed slow wave modulator as well as scanning electronic microscope (SEM) images of the fabricated structure. Slow light interacting with the variable concentration of free carriers is the effect intended to be used as a means to increase light-matter interactions, thus enhancing the modulation efficiency. Figure 3-53 (c), (d) show also the schematic of the designed slow wave modulator as well as scanning electronic microscope (SEM) images of the fabricated structure. Optical phase modulation in the slow wave waveguide is converted into amplitude modulation via the use of an asymmetric MZI. The SWPS is placed in the shorter arm of the MZI while a conventional rib waveguide with the same length and doping conditions is placed in the longer arm as a reference. This configuration allowed us to extract both phase shift variations and insertion losses as a function of the group index from the measured transmission spectra. 
(a)

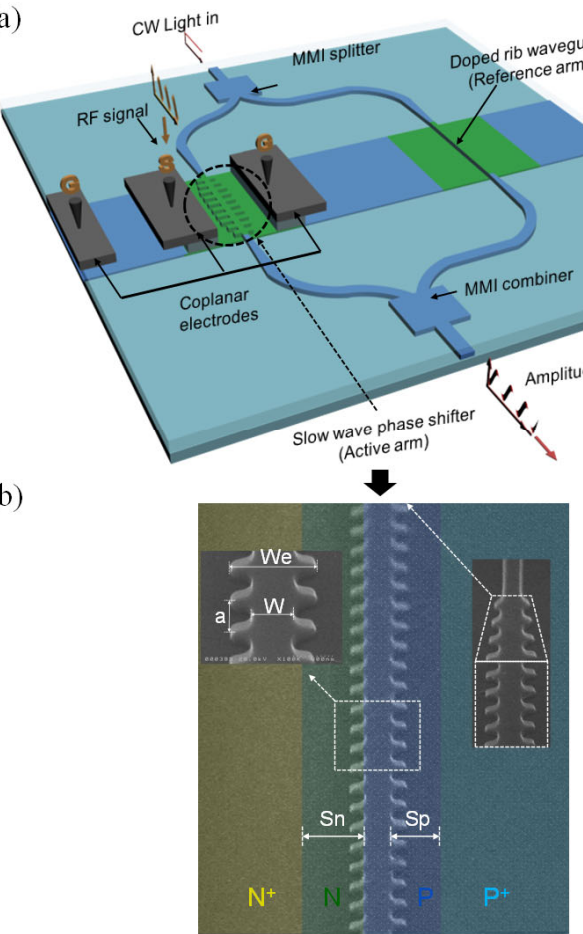

(c)

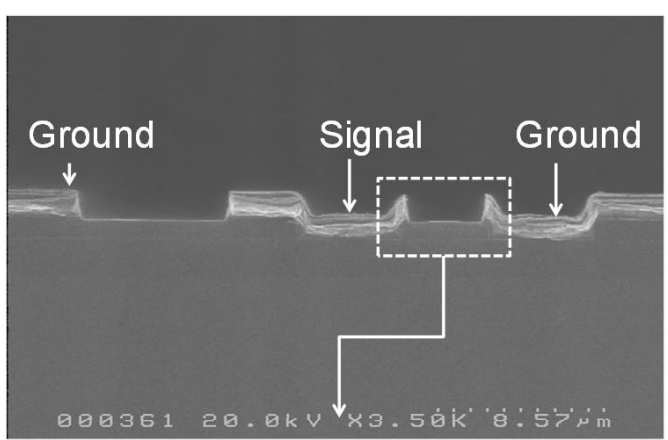

(d)

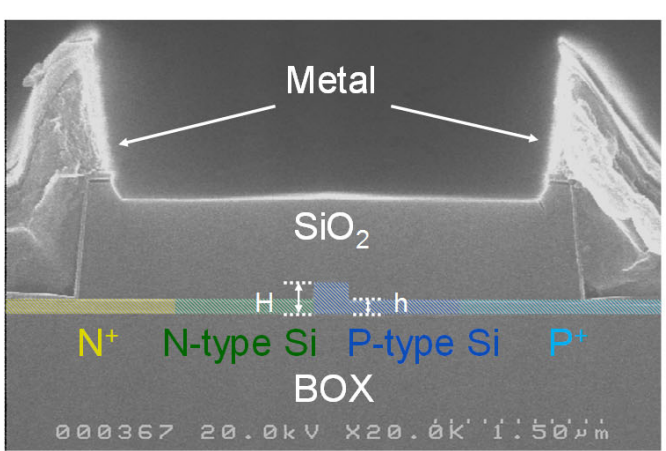

Figure 3-53: (a) Schematic of the modulator based on an asymmetric Mach-Zehnder interferometer (MZI). Multi-mode interference (MMI) structures are used to split and combine the light respectively at the input and output of the MZI. (b) Transverse scanning electron microscope (SEM) picture of the slow wave modulator with an overview of the ground-signal-ground coplanar (GSG) metal electrodes. (c), (b) Top and transverse SEM pictures of the corrugated waveguide. Left inset shows a zoomed view of the taper used to reduce the coupling losses at the rib to slow wave waveguide transitions. Right inset depicts a zoomed view of the corrugated waveguide. Doping regions are delimited by the colored areas.

\subsubsection{DC measurements}

As previously mentioned, optical phase modulation in the slow wave waveguide is converted into amplitude modulation through the use of an asymmetric MZI. Figure 3-54 (a), (b) show the MZI slow wave modulator normalized transmission spectra under varying reverse bias voltage. The device contains a 1mm-long slow wave phase shifter. The free spectral range (FSR) variations across wavelength, depicted in Figure 3-54 (c), can be used to extract the group index of the slow wave waveguide [70] using Equation (4.21). 


$$
n_{g}(\lambda)=\left(\frac{n_{\text {gref }, 1} \cdot L_{r e f, 1}+n_{\text {gref }, 2} \cdot \Delta L}{L_{c o r r}}\right) \pm \frac{\lambda^{2}}{F S R \cdot L_{\text {corr }}}
$$

Where again $\lambda$ is the central wavelength of the FSR, $n_{g}, n_{g}, r e f, 1$ and $n_{g}$,ref,2 are the respective group indices in the slow wave, $300 \mathrm{~nm}$ wide reference rib and $400 \mathrm{~nm}$ wide rib waveguides, $L_{c o r r}$ and $L_{r e f, 1}$ are the lengths in the slow wave and reference rib waveguides and $\Delta L$ stands for the length difference between the MZI arms considering only the $400 \mathrm{~nm}$ wide rib waveguide. The parameter values are $L_{\text {corr }}=L_{r e f, 1}=1 \mathrm{~mm}, \Delta L=0.9 \mathrm{~mm}, n_{g, r e f, 1} \sim 3.5$ and $n_{g, r e f, 2} \sim 3.7$. The selection of the sign in the right-hand side of Equation (4.21) depends upon the considered wavelength range and is related to the fact that the FSR exhibits different behaviors as we move from the fast light region towards the photonic band gap.

In the fast light regime (far enough from the photonic band gap) the separation between the interference fringes remains approximately constant, which is consistent with a nearly unvarying group index value and the negative sign must be used in Equation (4.21). However, as we enter the slow light regime, i.e. as we move closer to photonic band gap, the FSR increases up to a maximum value, which means that the phase difference between the MZI arms is minimized. From this point forward, a positive sign must be used in Equation (4.21) because higher group indices imply that the effective optical path length of the shorter arm become larger than that of the longer arm, thus causing the FSR to decrease.

It is important to notice the group index is assumed to be approximately constant over the wavelength range covered by the FSR. As this assumption is less accurate for wavelengths close to the photonic band gap, more precise results can be obtained considering only the difference between the maxima and minima of the transmission spectra, i.e. FSR/2. As can be seen, the maximum calculated group index value lies around 22. Furthermore, Figure 3-54 (d) depicts the resultant phase shift variations relative to $\pi$ radians versus applied reverse bias voltages and for varying group index. For completeness, the produced wavelength shift when a reverse bias voltage $V$ is applied can be estimated from

$$
\lambda(V) \approx \lambda(0)-\Delta \varphi(V) \frac{F S R}{2 \pi}
$$

where $\lambda(V)$ is the wavelength shifted from $\lambda(0)$ for a given applied voltage, as a result of the $\Delta \varphi(V)$ phase variation in the slow wave phase shifter. According to 
Equation (4.20) and (4.22), and in agreement with the results shown in Figure 3-54 (a) (b), the transmission spectrum is blue shifted for wavelengths where the corresponding group index of the slow wave waveguide does not differ too much from that of the rib waveguide so the effective propagation length in the longer MZI arm is larger than in the shorter arm. At wavelengths where the FSR tends to infinite, which takes place around $1561 \mathrm{~nm}$, the spectrum is no longer shifted, since the MZI behaves as a symmetric structure, and only the transmitted power is altered, as also clearly seen in Figure 3-54 (a) (b). For wavelengths below $1561 \mathrm{~nm}$, the transmission spectrum is in turn red shifted due to the longer optical path length of the shorter MZI arm.
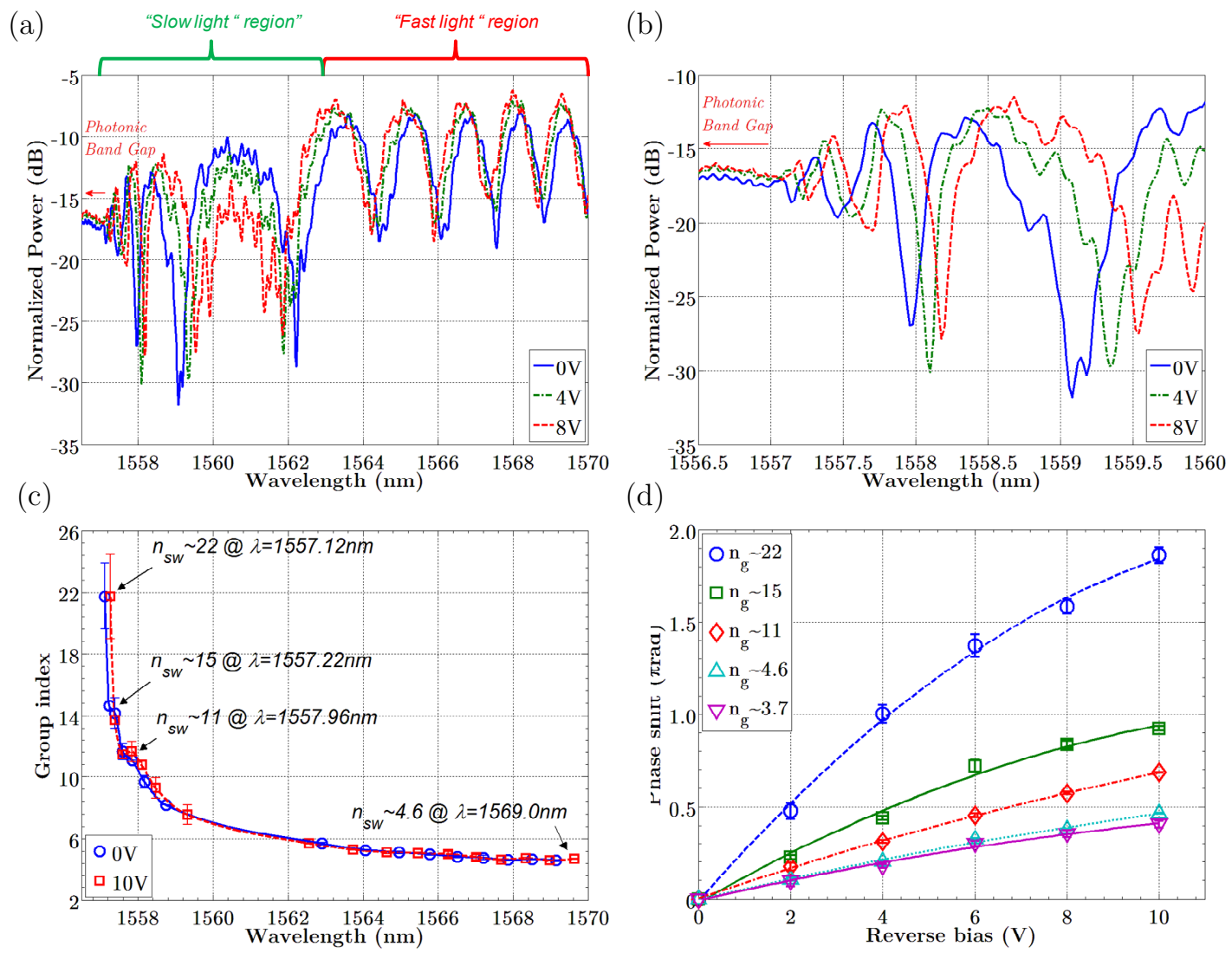
(d)

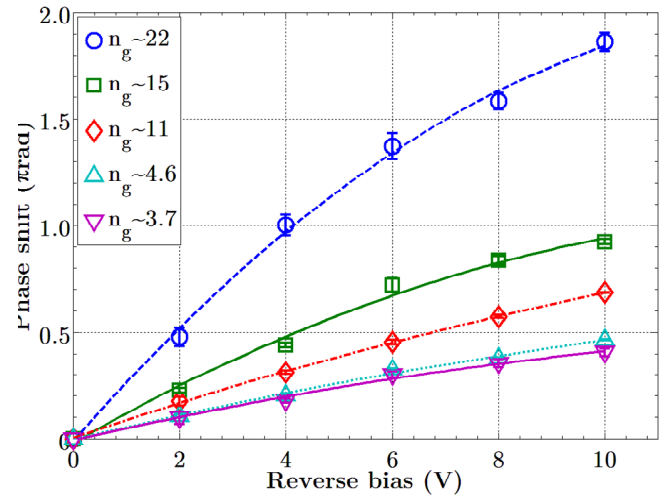

Figure 3-54: (a) Normalized transmission spectrum of the slow wave modulator for three different reverse biased voltages. The slow and fast light regions are respectively delimited in green and red. (b) Expanded view of the slow light region. (c) Group index variation versus wavelength at $0 \mathrm{~V}$ and $10 \mathrm{~V}$ bias voltages. (d) Phase shift dependence versus applied bias for varying group index.

As a reference and to demonstrate the enhancement produced by the slow wave effect, a conventional 400nm wide silicon rib phase shifter exhibiting a group index of $\sim 3.7$ and embedded in the same $p n$ junction with identical doping 
concentrations has been included on the same sample and characterized. As observed in Figure 3-54 (d), the corresponding achieved phase shift is approximately the same as that of the slow wave phase shifter biased in the fast light regime, but strongly increases as the group index does within the slow light region. This is because the modulation efficiency $V_{\pi} L_{\pi}$ of the slow wave phase shifter, calculated as the average value between 0 and $10 \mathrm{~V}$ (with $2 \mathrm{~V}$ increments), is significantly enhanced. Indeed, although for the conventional phase shifter, the calculated $V_{\pi} L_{\pi}$ is $\sim 2.2 \mathrm{~V} . \mathrm{cm}$, it significantly improves for the slow wave phase shifter, even for moderate group indices. For instance, a $V_{\pi} L_{\pi} \sim 1.27 \mathrm{~V}$.cm is obtained for a group index of 11 and as low as $\sim 0.45 \mathrm{~V}$.cm when the group index increases up to $\sim 22$.

In principle, a higher phase shift variation for a given voltage should give rise to a larger shift of the transmission spectrum. However, the modulation efficiency should always be evaluated relative to the FSR and not in terms of absolute wavelength shift. In the slow wave waveguide, a higher effective index variation is achieved when the group index increases, so the wavelength shift variation with respect to the FSR must increase accordingly. This behavior can be clearly observed in Figure 3-54 (a), (b). Indeed, as we move towards the photonic band edge, the FSR becomes narrower but the wavelength shift relative to the FSR visibly increases hence demonstrating the modulation efficiency enhancement achieved in the slow wave region.

\section{Interval of confidence for phase shift measurements}

For completeness, error bars were also assigned to the calculated phase shift against voltage curves. The uncertainty on the phase shift calculation originates essentially from the FSR determination which may be impaired by FP ripples. As in the case of the group index, the error bars cover the maximum uncertainty on the FSR estimation for each applied voltage.

\subsubsection{Enhancement versus insertion loss penalty}

The enhanced modulation efficiency offers two alternatives over the conventional rib modulator. Namely, either a device requiring lower drive voltage while conserving the modulation length or a more compact device requiring the same drive voltage as already discussed in section3.4.4. Here, we have selected the second option for several reasons besides the compactness. Indeed, a shorter 
modulation length is expected to increase the modulation bandwidth and reduce the insertion losses. The latter is in fact the main limitation of slow wave-based devices as increasing the group index will lead to higher losses. Briefly, although their causes and mechanisms have been subject of discussions [70, 105-108], one may point out coupling and slow wave propagation as the main source of losses. Coupling losses originates from the modal mismatch between the fast and slow modes at the rib to slow wave waveguide transitions. Furthermore, the higher propagation losses of slow wave modes are a consequence of the stronger interaction of the optical mode with, on one hand fabrication imperfections (essentially sidewall roughness and residual crystalline defects after the implantation process) and, on the other hand free carriers generating higher absorption. Backscattering loss produced by disorder is thought to play a minor role owing to the moderately high group index involved here.

The insertion losses shown in Figure 3-55 (a) can be estimated from the ER variations of the interference fringes using a specific method, here called method 1, and described in details elsewhere [127]. As an alternative approach, here called method 2, they have also been directly estimated from the measured transmission spectrum of a single slow wave phase shifter. The two methods show good agreement as depicted in Figure 3-55 (a). The insertion loss dependence upon group index has been approximated by a linear fitting, which increases from $\sim 5 \mathrm{~dB}$ in the fast light region $\left(n_{g} \sim 4.6\right)$, to around $16 \mathrm{~dB}$ at $n_{g} \sim 11$ and $34 \mathrm{~dB}$ at $n_{g} \sim 22$. However, although there is a significant increase in loss inherent in the use of higher group indices, the total insertion loss penalty in our slow wave modulator with respect to a conventional device may be balanced owing to the fact that smaller modulation lengths are required to achieve a given phase shift. To illustrate this, an enhancement factor has been defined as

$$
E F \approx \frac{\Delta \varphi_{s w}}{\Delta \varphi}
$$

where $\Delta \varphi_{s w}$ and $\Delta \varphi$ are the respective average phase shifts achieved in the slow wave and conventional phase shifters. The enhancement factor, directly related to the modulation efficiency $V_{\pi} L_{\pi}$, is shown in Figure 3-55 (b) together with the corresponding modulation efficiency for each group index value. The insertion loss penalty of the slow wave modulator compared to the conventional modulator can be defined as

$$
I L_{\text {Penalty }}=C_{p}+\left(\alpha_{\text {corr }}-\alpha \cdot E F\right) \cdot L_{\text {corr }}
$$


where $C_{p}$ are the total coupling losses at the input and output of the slow wave waveguide, $\alpha_{\text {corr }}$ and $\alpha$ are the respective propagation losses in the slow wave and conventional rib waveguides, $E F$ is the enhancement factor and $L_{\text {corr }}$ is the modulation length. From Fig. $2 \mathrm{~F}, L_{\text {corr }}=1 \mathrm{~mm}, \alpha=4.5 \mathrm{~dB} / \mathrm{mm}$ and the term $C_{p}+$ $\alpha_{\text {corr }} \cdot L_{\text {corr }}$ is basically equal to the results shown in Figure 3-54 (b).

(a)

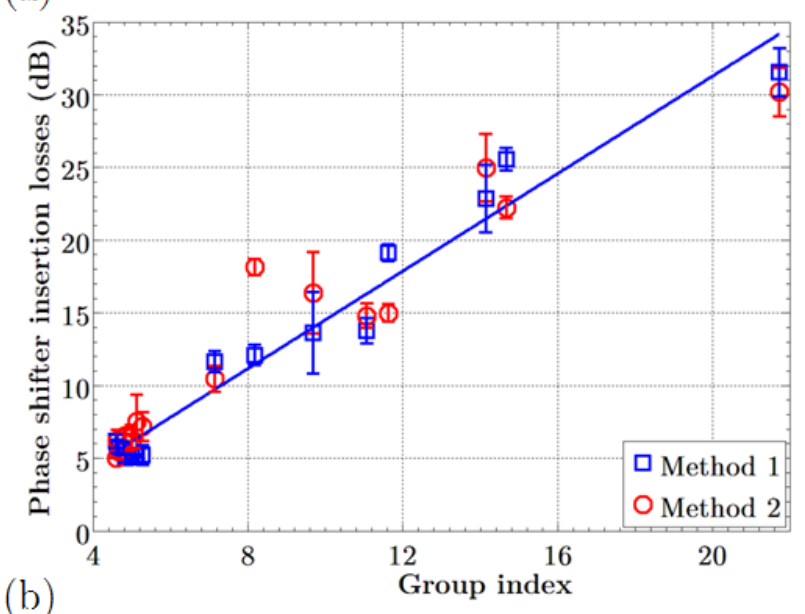

(b)

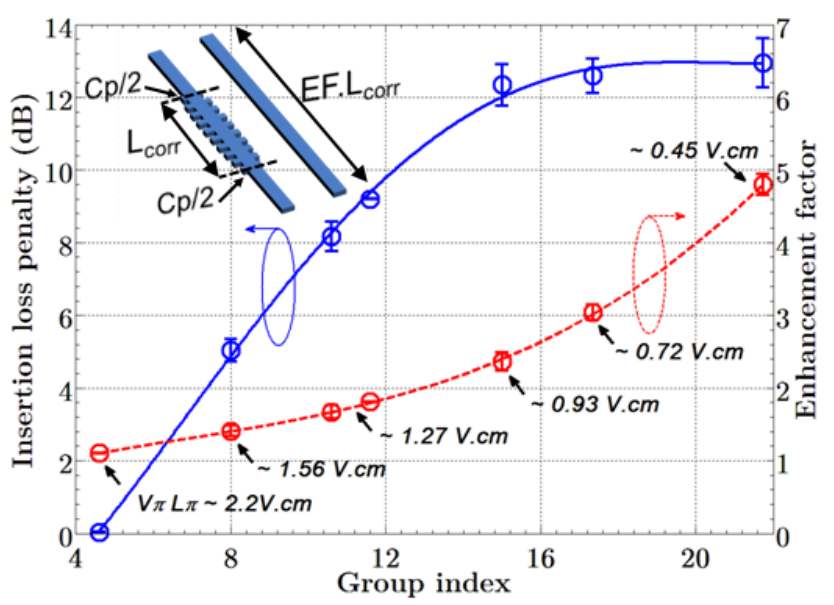

Figure 3-55: (a) Insertion losses versus group index of the $1 \mathrm{~mm}$ long slow wave phase shifter (b) Insertion loss penalty relative to the conventional modulator. $C_{p}$ accounts for the coupling losses between the slow wave and conventional rib waveguides while $L_{\text {corr }}$ $=1 \mathrm{~mm}$ is the modulation length. Average modulation efficiencies $V_{\pi} L_{\pi}$ and enhancement factor (between 0 and $10 \mathrm{~V}$ ) versus group index of the slow wave modulator are shown. $V_{\pi}$ and $L_{\pi}$ are respectively the bias voltage and modulation length required to achieve a $\pi$ radian phase shift.

It can be seen that the insertion loss penalty linearly increases up to $13 \mathrm{~dB}$ for a group index of $\sim 16$ but then appears to slightly begin to drop for higher group indices. This is because the enhancement factor improvement dominates 
over the loss increase and suggests that the insertion loss penalty could be reduced by using very high group indices if this trend were preserved. However, it has been reported [108] that the losses drastically increase for group indices above $\sim 30$. This would reverse the suggested trend and yield to a local minimum of insertion loss penalty associated with a maximum enhancement factor. On the other hand, very high group indices would also lead to a strong dependence of the phase shift upon applied voltage as the group index variation with voltage could no longer be considered constant at a given wavelength.

In any case, the modulator length may be decreased to reduce the insertion losses which in turn would also improve the modulator 3dB roll-off bandwidth although at the expense of a lower modulation depth. The smaller length would also reduce the insertion loss penalty, although it is worth noting that this reduction could be limited if the coupling losses, which are obviously independent upon the length, dominated over the propagation losses.

\subsubsection{Small signal RF measurements}

In an ideal TW electrode design terminated with a matching impedance (no reflection at the output), the electrical modulating signal co-propagates with the optical signal at similar speeds. However, it is clear that slowing down light is likely to produce some changes in the $3 \mathrm{~dB}$ roll-off bandwidth values as discussed in section 3.4.5. To provide experimental evidence of the influence of the modulation length over the bandwidth and modulation depth, two $1 \mathrm{~mm}$ and $0.5 \mathrm{~mm}$ long slow wave MZI modulators, with respective footprints of 650 $\left(1000 \mathrm{x} 0.650 \mu \mathrm{m}^{2}\right)$ and $325 \mu^{2}\left(1000 \mathrm{x} 0.325 \mu \mathrm{m}^{2}\right)$ have been characterized.

Transverse electric (TE) polarized light emitted by an external cavity laser (ECL) is injected into the modulator via grating couplers. The transmitted power is optimized through the use of a polarization controller (PC). The small electrical signal delivered by the port 1 of the RF vectorial network analyzer (VNA Agilent E5071C) is coupled a DC bias via a bias-Tee and applied through high speed GSG probes to the travelling wave electrodes terminated externally by a $50 \Omega$ load coupled to a DC block. In addition, a $6 \mathrm{~V}$ DC bias was applied to drive the slow wave MZI modulator approximately at quadrature. The output modulated signal is amplified with an erbium-doped fiber amplifier (EDFA) and photodetected by a high speed photodiode (XPDV2040R $50 \mathrm{GHz}$ ) connected to 
the port 2 of the RF VNA in order to extract the electro-optical S21 transfer function.

Figure 3-56 (a) shows the normalized electro-optical frequency response of the two MZI slow wave modulators both driven at a group index value of $\sim 11$. It can be seen that the $3 \mathrm{~dB}$ roll-off frequency is improved from $\sim 11 \mathrm{GHz}$ to $\sim 16 \mathrm{GHz}$ due to the length reduction of the slow wave phase shifter. In fact, the bandwidth is mainly limited as a result of the velocity mismatch between the electrical and optical signals as the travelling wave (TW) electrode parameters are not properly optimized for the slow light regime. Indeed, as shown in Figure 3-56 (a), a bandwidth higher than $20 \mathrm{GHz}$ was measured in the fast light region for the $1 \mathrm{~mm}$ long phase shifter, but declines as the group index increases thus imposing a trade-off between bandwidth and modulation efficiency. Additionally, the reduction of the modulation length provides a higher bandwidth owing to the lower influence of the velocity mismatch.

(a)

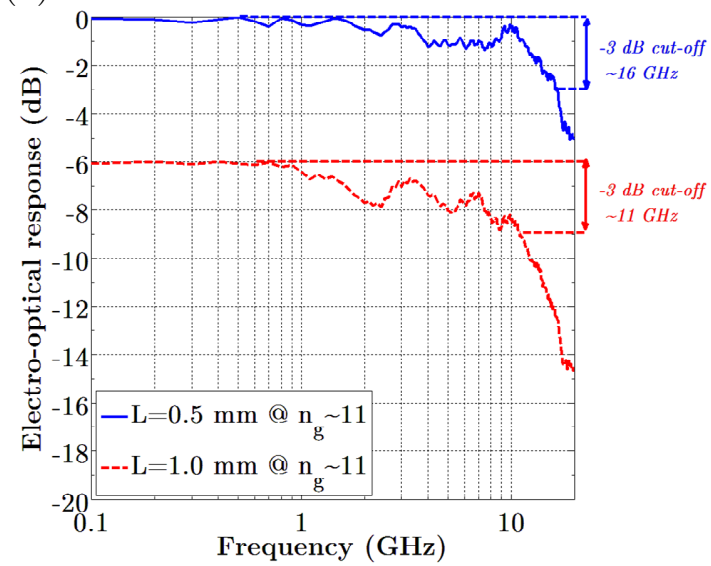

(b)

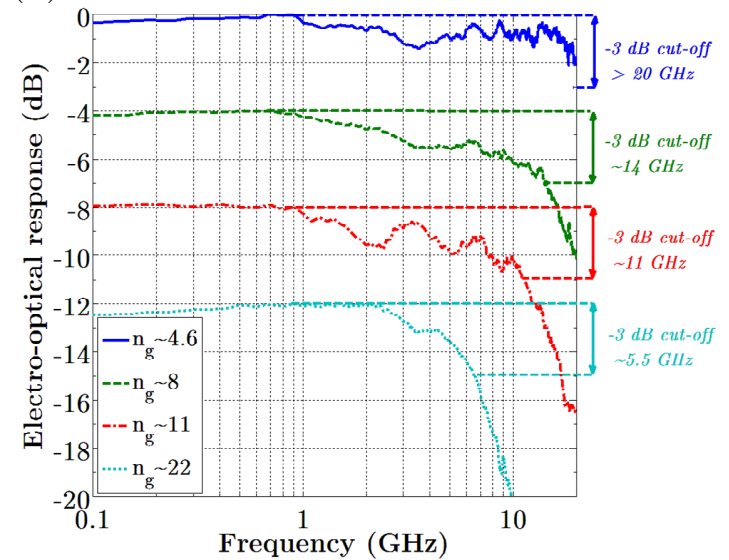

Figure 3-56: Slow wave modulator electro-optical response. (a) Normalized electro-optical frequency response of two MZI modulators with respective slow wave phase shifter lengths of $1 \mathrm{~mm}$ and $0.5 \mathrm{~mm}$. Spectra are shifted vertically by $6 \mathrm{~dB}$ for clarity. (c) Normalized electro-optical frequency response of the $1 \mathrm{~mm}$ long slow wave modulator for varying group index. Spectra are shifted vertically by $4 \mathrm{~dB}$ for clarity. The $3 \mathrm{~dB}$ roll-off bandwidth of the TW slow wave modulator is mainly limited as a result of the velocity mismatch between the electrical and optical signals, which imposes a trade-off between bandwidth and modulation efficiency

To provide further evidence of the fact that the slow wave modulator $3 \mathrm{~dB}$ roll-off bandwidth is essentially limited by the group index increase, we proceeded 
to additional measurements. Because the electrical signal transmitted to the modulator is highly dependent upon the contact quality between the RF probes and the electrodes as well as in order to guarantee that the travelling wave transmission line is well matched to the $50 \mathrm{ohm}$ termination, S11 electrical measurements were simultaneously performed along with S21 electro-optical characterization. For completeness and to evaluate the electrode losses produced by the skin effect, the S21 electrical coefficient of the modulator was carried out. The setup calibration was performed using an impedance standard substrate provided by the high speed probe seller. As shown in Figure 3-57, the S11 electrical responses of the $1 \mathrm{~mm}$ and $0.5 \mathrm{~mm}$ RF transmission line lie well below -10 $\mathrm{dB}$ over the considered modulation frequency range [0.1 to $20 \mathrm{GHz}]$. This clearly suggests that very low signal reflection takes place and therefore the slow wave modulator behavior is not limited by RF impedance mismatch within the latter range.

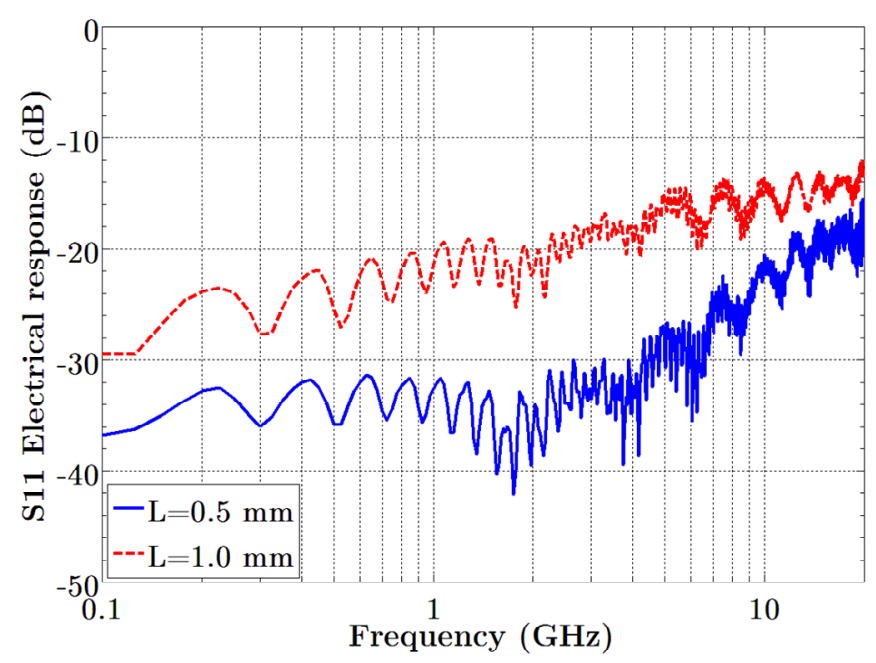

Figure 3-57: S11 Electrical response of the $1 \mathrm{~mm}$ and $0.5 \mathrm{~mm}$ RF transmission line

In addition to the S11 response, S21 small electrical signal measurements were realized to estimate the CPWG electrode losses caused by the skin effect. Here, the electrode attenuation is evaluated by measuring four different lengths available on the fabricated samples. As expected, the transmitted electrical power against frequency decreases in a sharper manner as the electrode length increases as shown in Figure 3-58 (a). Just as for silicon photonic waveguides, the electrode losses are then calculated by cut-back leading to the results illustrated in Figure 3-58 (b). As seen, the losses are respectively $0.29 \mathrm{~dB} / \mathrm{mm}, 0.72 \mathrm{~dB} / \mathrm{mm}, 1.13$ $\mathrm{dB} / \mathrm{mm}$ and $2.35 \mathrm{~dB} / \mathrm{mm} @ 1 \mathrm{GHz}, 5 \mathrm{GHz}, 10 \mathrm{GHz}$ and $20 \mathrm{GHz}$. This squareroot-like trend against frequency is typical of a conductor loss dependency caused 
by the skin effect, (i.e. when the current tends to flow close to the surface) as shown by the following Equation:

$$
\alpha_{c}=\alpha_{0} \cdot f^{1 / 2}
$$

Where $\alpha_{0}$ is the attenuation coefficient and $f$ the frequency.

(a)

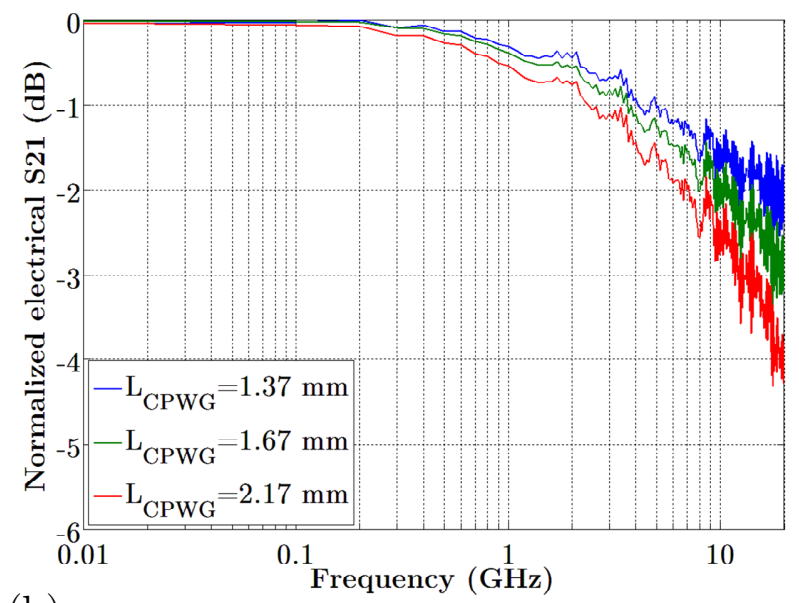

(b)

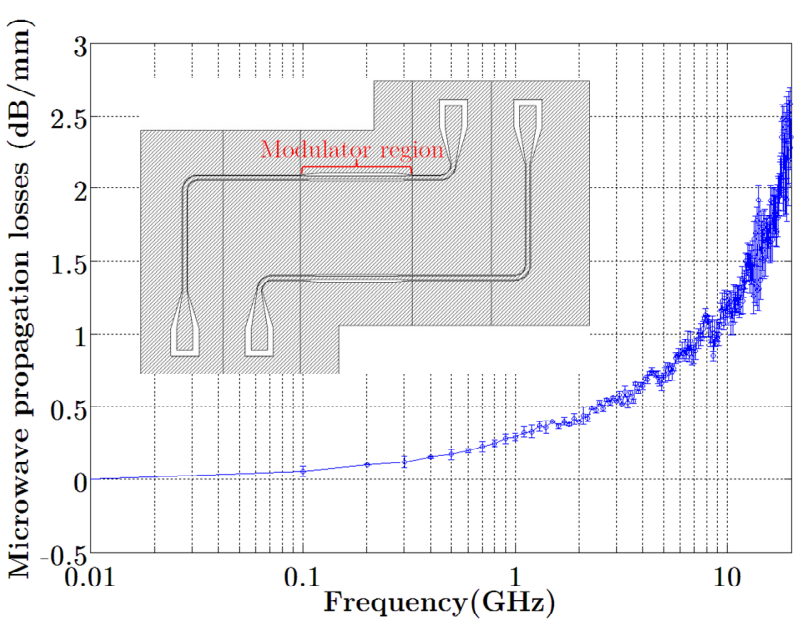

Figure 3-58: (a) Electrical response of the coplanar waveguide (CPWG) electrodes (S21) with varying length. (b) Radio-frequency propagation losses versus frequency calculated with the cut back method from (a)

The attenuation coefficient $\alpha_{0}$ may be calculated from the S21 transmission of various CPWG lengths plotted versus the square root of the frequency as depicted in Figure 3-59. The linear fit of the resulting transmission curves allows us to determine the corresponding linear equations in the form $y=a x+b$, whose slope $a$ is the actual attenuation coefficient multiplied by the CPWG lengths 
given in the legend of Figure 3-59. The attenuation coefficient of the $1.37 \mathrm{~mm}$, $1.67 \mathrm{~mm}$ and $2.17 \mathrm{~mm}$-long CPWG can therefore be derived as: $\alpha_{0}=a / L_{C P W G}$ yielding respectively to $\alpha_{0}=0.45,0.44$ and $0.39 \mathrm{~dB} \cdot \mathrm{mm}^{-1} \cdot \mathrm{GHz}^{-1 / 2}$.

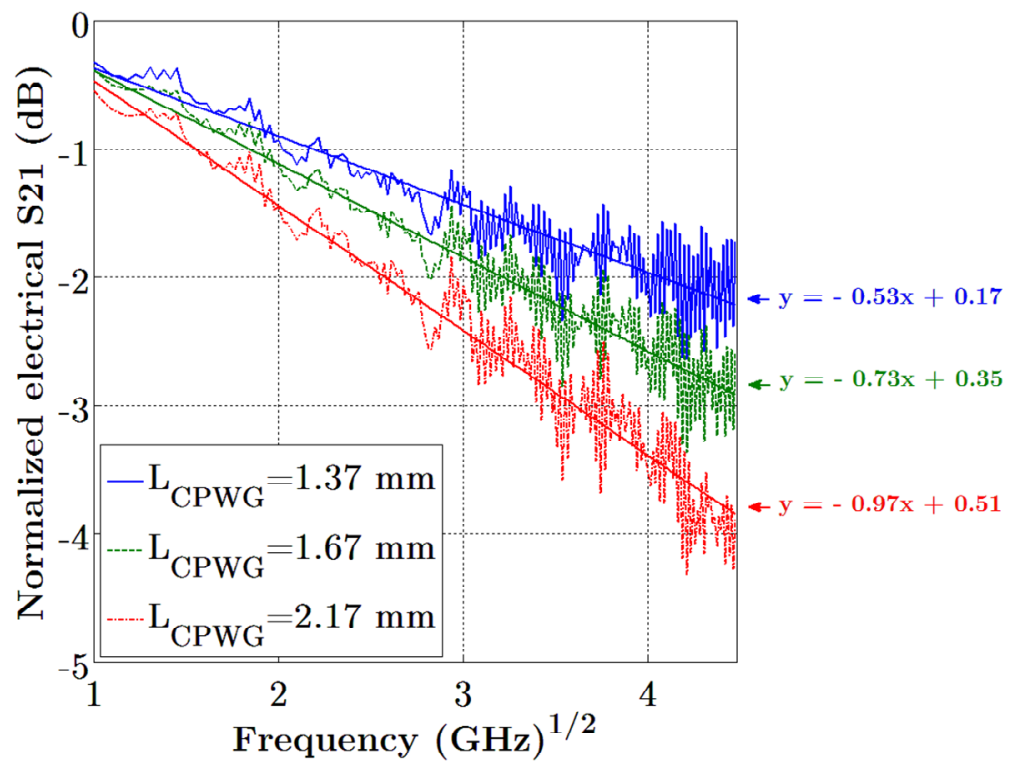

Figure 3-59: Normalized S21 plotted versus the square root of the frequency. The three electrical transmission curves can be approximated by linear fits, whose equations are given on the right hand side.

To cross-check, we may plot these results along with the microwave CPWG propagation loss dependency upon frequency calculated by cut-back. The three values of $\alpha_{0}$ worked out previously are included in Equation (4.25).

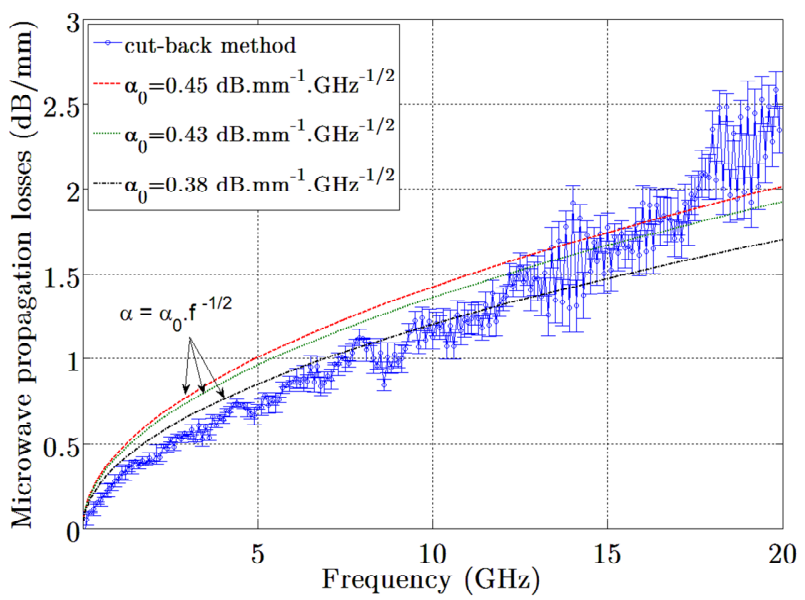

Figure 3-60: Microwave propagation losses versus frequency calculated via cut-back and through the equation modeling the loss variations caused by the skin effect. 
As seen, although the trend between the two methods is conserved, a slight discrepancy may be observed. Indeed, the uncertainty on the determination of the attenuation coefficient of the three coplanar transmission lines yields to slightly different results probably due to the non-optimum positioning of the probes coupled to the non-fully identical CPWGs. At higher frequency $(>16 \mathrm{GHz})$ the divergence between the two methods seems to increase. One may point out the dielectric loss influence within the low resistive regions (highly doped regions) as a possible candidate for such a difference[128]. Still, we may say that both methods are in reasonably good agreement within the error margin. Additionally, we can estimate the required microwave attenuation for our modulator not to be limited in terms of electro-optical bandwidth. It is highly desirable to maintain the electrical signal amplitude as high a possible until the end of the active region. Therefore, we may set the total microwave loss $\alpha=3 \mathrm{~dB}$, corresponding to a resultant amplitude signal attenuation of $30 \%$ (calculated as $10^{(-\alpha / 20)}$ ) at the end of line. As seen in Figure 3-61, the required attenuation coefficient corresponding to the $0.2,0.5$, and 1 mm-long CPWG transmission lines lie beyond the experimentally calculated value of around $0.4 \mathrm{~dB} \cdot \mathrm{mm}^{-1} \cdot \mathrm{GHz}^{-1 / 2}$ up to $40 \mathrm{GHz}$ (considering that the skin effect dominates over the remainder sources of losses), although as mentioned previously, the microwave losses seem to follow a supra square root tendency from frequency values $>16 \mathrm{GHz}$, which might well corresponds to dielectric loss. In any case, considering a coefficient of around 0.4 $\mathrm{dB} \cdot \mathrm{mm}^{-1} \cdot \mathrm{GHz}^{-1 / 2}$, the microwave losses on the $0.2,0.5$, and $1 \mathrm{~mm}$ CPWG transmission lines correspond to respective signal attenuations of $3 \%(0.25 \mathrm{~dB})$, $7 \%(0.6 \mathrm{~dB})$ and $14 \%(1.2 \mathrm{~dB})$ at $10 \mathrm{GHz}$ which leads us to conclude that at this frequency very low microwave attenuation takes place. The losses calculated at the remainder frequencies are represented in Table 3-9. Again, the attenuation calculations confirm that for the three considered CPWG lengths, the microwave losses lie below the initial specification of $3 \mathrm{~dB}$ attenuation as a maximum. Let us remind however that beyond $20 \mathrm{GHz}$, we made the assumption that the skin effect keep dominating over any other source of losses. Nevertheless, as the CWPG length increase up to $3 \mathrm{~mm}$, the microwave losses increase accordingly. 


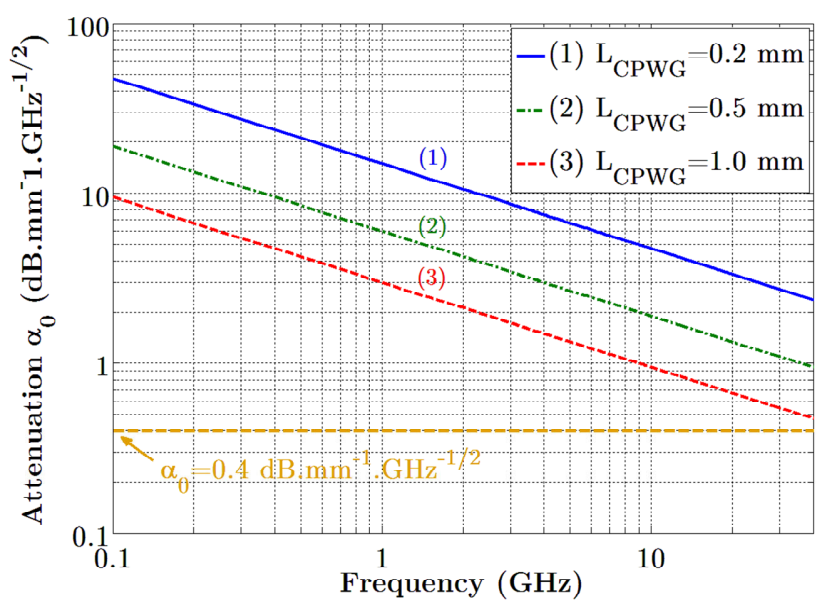

Figure 3-61: Required microwave attenuation coefficient versus frequency for different CPWG length to maintain a maximum of $3 \mathrm{~dB}$ attenuation of the transmission line. The orange dashed line stands for the experimental attenuation coefficient calculated previously.

For instance, at $20 \mathrm{GHz}$, almost half of the signal amplitude is lost at the end of the transmission line. At higher frequencies, (up to $40 \mathrm{GHz}$ ) more than $50 \%$ of the effective applied voltage is attenuated. This leads us to conclude that the transmission line length should be kept as short possible, as long as the modulator efficiency enables such a configuration. This reinforces the relevancy of using slow wave structure to get rid of the potential microwave losses, which may be a strong limiting factor for high speed operation. Overall we have shown that the CPWG losses are unlikely to be responsible for the bandwidth limitations of our SW EOM a least up to $20 \mathrm{GHz}$. This confirms the initial assumption that the electro-optical bandwidth is rather limited by the group velocity mismatch between the optical and electrical signals. 


\begin{tabular}{|c|c|c|c|}
\hline CPWG length $(\mathbf{m m})$ & Frequency $(\mathbf{G H z})$ & Attenuation $(\mathbf{d B})$ & Attenuation (\%) \\
\hline 0,2 & 10 & 0,25 & 3 \\
\hline 0,2 & 20 & 0,36 & 4 \\
\hline 0,2 & 30 & 0,43 & 5 \\
\hline 0,2 & 40 & 0,5 & 7 \\
\hline 0,5 & 10 & 0.6 & 10 \\
\hline 0,5 & 20 & 0,9 & 12 \\
\hline 0,5 & 30 & 1,1 & 14 \\
\hline 0,5 & 40 & 1,3 & 13 \\
\hline 1 & 10 & 1,3 & 18 \\
\hline 1 & 20 & 1,8 & 22 \\
\hline 1 & 30 & 2,2 & 25 \\
\hline 1 & 40 & 2,5 & 35 \\
\hline 3 & 10 & 3,8 & 46 \\
\hline 3 & 20 & 5,4 & 53 \\
\hline 3 & 30 & 6,6 & 58 \\
\hline 3 & 40 & 7,6 & \\
\hline
\end{tabular}

Table 3-9: Microwave attenuation at different frequencies for varying CPWG length.

The corresponding attenuation coefficient of the CWPG transmission line is $\alpha_{0}=0.4$ $\mathrm{dB} \cdot \mathrm{mm}^{-1} \cdot \mathrm{GHz}^{1 / 2}$

\subsubsection{Eye diagrams and bit error rate measurements}

Electro-optical bandwidth improvement indicates that the maximum data rate our slow wave modulator could handle without degrading the BER performance is increased accordingly. Data transmission measurements were carried out driving the slow wave modulator with a non-return to zero NRZ PRBS of length $2^{31}-1$ delivered by a bit pattern generator (SHF BPG 44E) connected to an external clock. The electrical signal was amplified through a $40 \mathrm{GHz}$ driving amplifier to achieve a voltage swing of $\sim 5 \mathrm{~V}_{\mathrm{pp}}$ and combined to a $6 \mathrm{~V}$ DC bias voltage using a bias-tee. In line with our previous electro-optical measurements, the modulating signal was applied to the TW electrodes terminated externally by a $50 \Omega$ resistance coupled to a DC block as illustrated in Figure 3-62. The output modulated optical signal was then photodetected by a $40 \mathrm{GHz}$ Digital Communication Analyzer (Infiniium DCA-J 86100C). The BER was measured using an Error Analyzer (SHF EA 44) and evaluated as a function of the optical power received at the photodetector. 


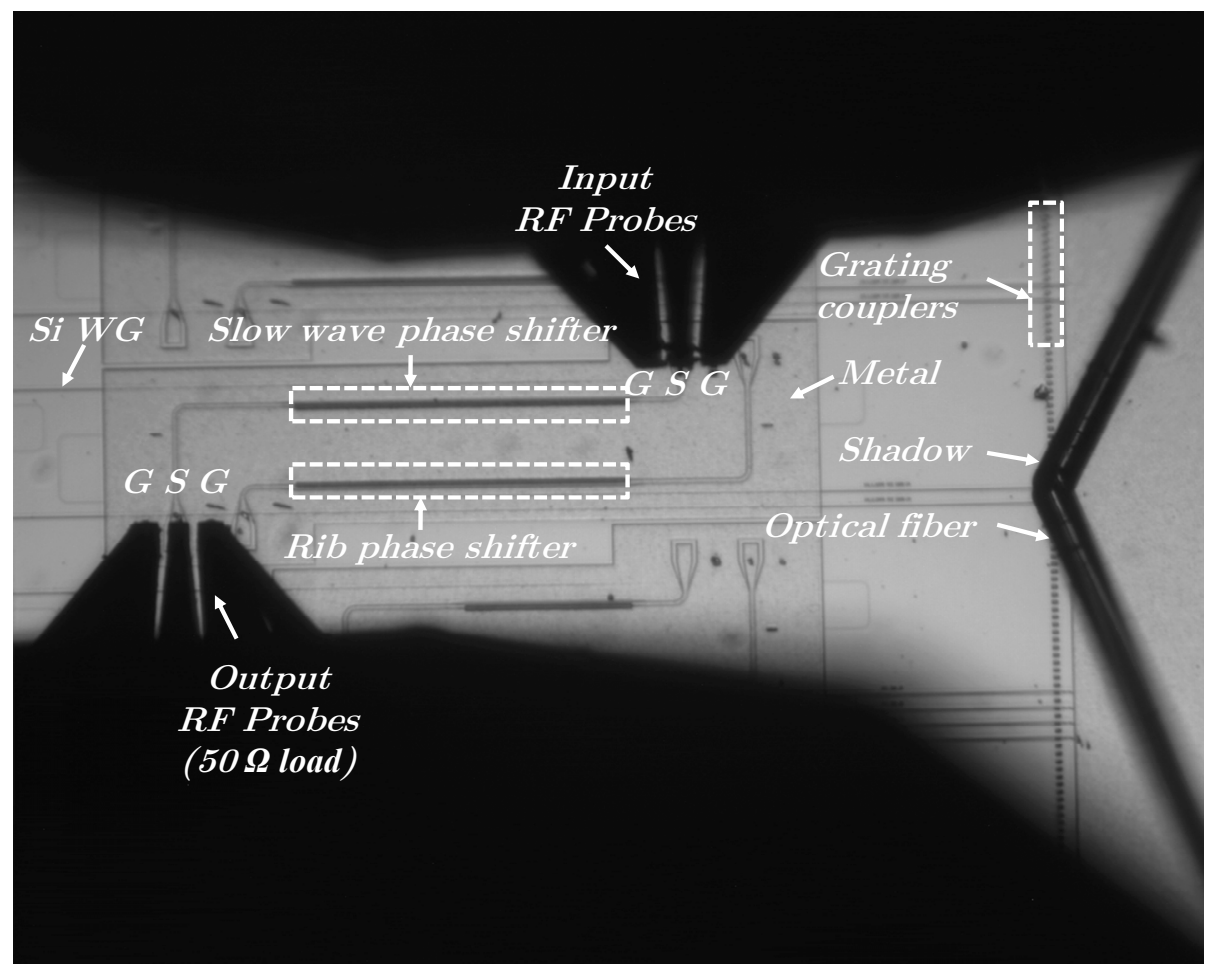

Figure 3-62: Optical microscope image of the slow wave modulator under test. The slow wave phase shifters are $500 \mu \mathrm{m}$ long.

The $1 \mathrm{~mm}$ and $0.5 \mathrm{~mm}$ long slow wave modulator eye diagrams at $10 \mathrm{~Gb} / \mathrm{s}$, $20 \mathrm{~Gb} / \mathrm{s} 30 \mathrm{~Gb} / \mathrm{s}$ and $40 \mathrm{~Gb} / \mathrm{s}$ are depicted respectively in Figure 3-63 (a),(b),(c) d),(e),(f). In all cases the eye patterns were measured under identical experimental conditions. In agreement with the measured $1 \mathrm{~mm}$ long modulator roll-off frequency, the photodetected optical signal is distorted beyond $10 \mathrm{~Gb} / \mathrm{s}$. Due to the longer rise and fall times, the eye pattern closes up leading to an increasing intersymbol interference (ISI). In addition, the ER is also affected and rapidly decreases from $9.5 \mathrm{~dB}$ at $10 \mathrm{~Gb} / \mathrm{s}$ to $6.7 \mathrm{~dB}$ and $4.9 \mathrm{~dB}$ at $20 \mathrm{~Gb} / \mathrm{s}$ and $30 \mathrm{~Gb} / \mathrm{s}$, respectively. On the other hand, for the $0.5 \mathrm{~mm}$ long slow wave modulator, the eye pattern remains relatively well open at both $10 \mathrm{~Gb} / \mathrm{s}$ and $20 \mathrm{~Gb} / \mathrm{s}$ and noticeable ISI can only be observed at $30 \mathrm{~Gb} / \mathrm{s}$. As expected, the ER is reduced compared to the $1 \mathrm{~mm}$ long slow wave modulator due to the lower achieved phase shift but is in turn less degraded by ISI as the bit rate increases. The measured ERs are $7.6 \mathrm{~dB}, 6.3 \mathrm{~dB}$ and $5.3 \mathrm{~dB}$ at $10 \mathrm{~Gb} / \mathrm{s}, 20 \mathrm{~Gb} / \mathrm{s}$ and $30 \mathrm{~Gb} / \mathrm{s}$ respectively. Increasing the speed further up to $40 \mathrm{~Gb} / \mathrm{s}$ shows that the modulator keeps responding to the electrical driving signal in spite of a noticeable degradation of the eye diagram. The estimated ER at this rate is $\sim 3 \mathrm{~dB}$. 

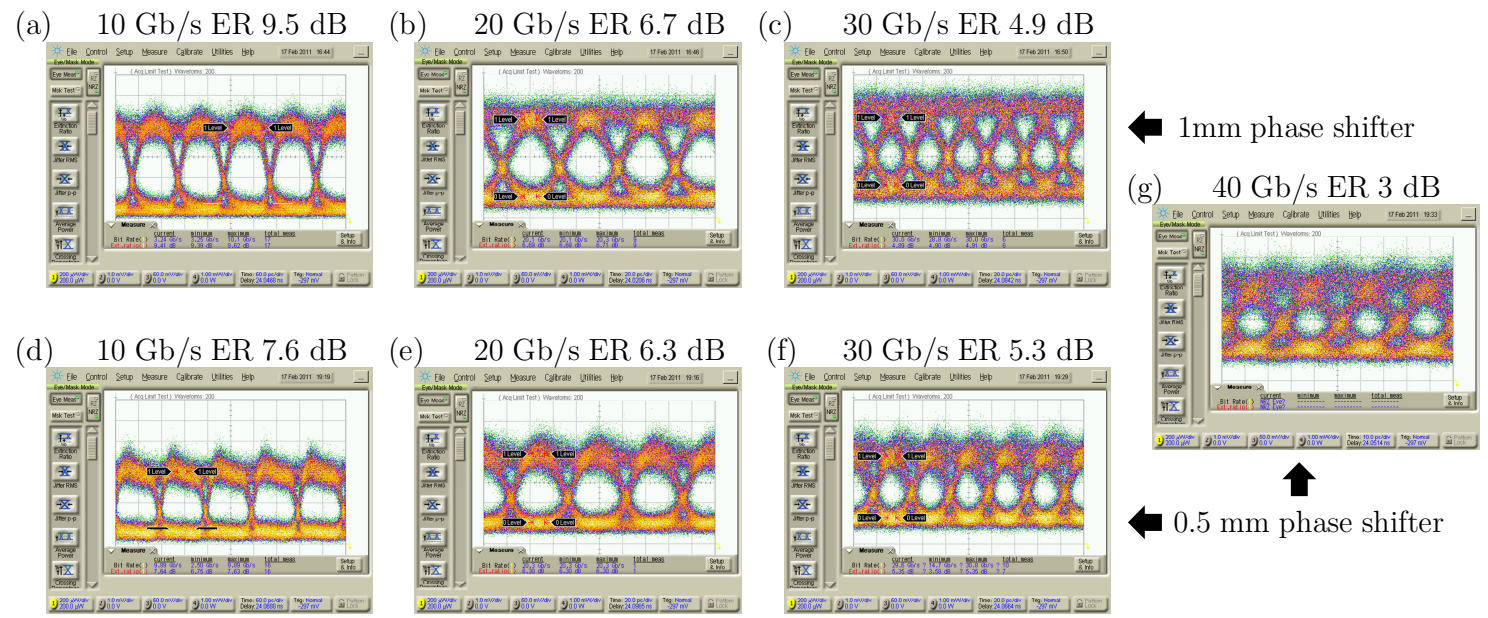

Figure 3-63: (a)-(f), Eye diagrams at (a) resp. (d) $10 \mathrm{~Gb} / \mathrm{s}$, (b) resp. (e) $20 \mathrm{~Gb} / \mathrm{s}$ and (d) resp. (e) $30 \mathrm{~Gb} / \mathrm{s}$ resp. (f) and (g) $40 \mathrm{~Gb} / \mathrm{s}$ of the $1 \mathrm{~mm}$ resp. $0.5 \mathrm{~mm}$ long slow wave modulators.

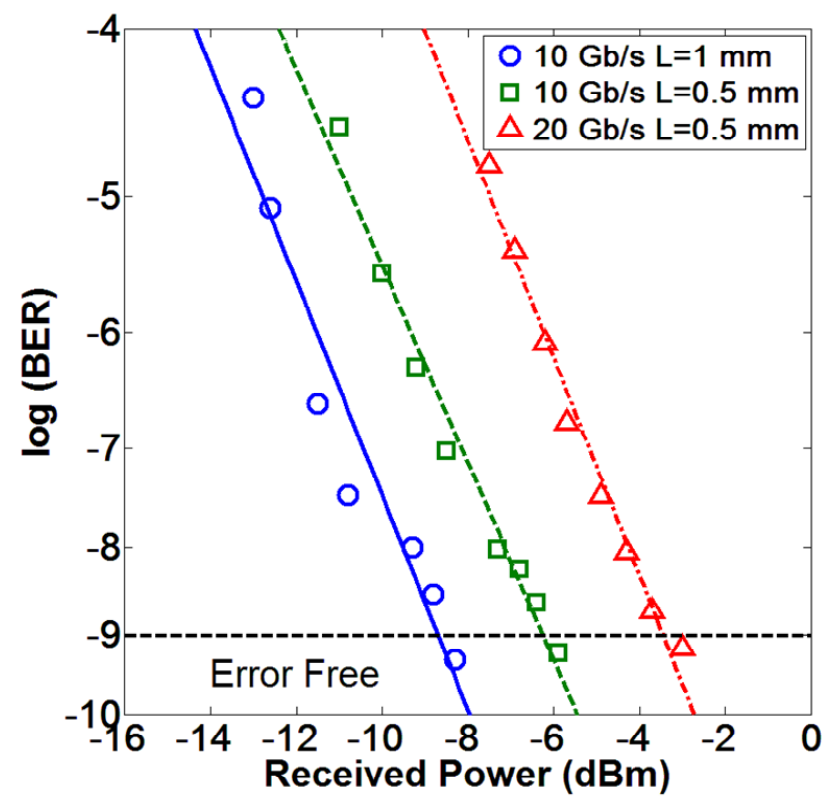

Figure 3-64: Bit-error rate (BER) curves at $10 \mathrm{~Gb} / \mathrm{s}$ and respectively $10 \mathrm{~Gb} / \mathrm{s}$ and 20 $\mathrm{Gb} / \mathrm{s}$ for the $1 \mathrm{~mm}$ and $0.5 \mathrm{~mm}$ long slow wave modulators.

The BER was also measured to characterize thoroughly the data transmission capability of our slow wave devices. Figure 3-63 (h) shows the BER results with interpolated linear curves at $10 \mathrm{~Gb} / \mathrm{s}$ and both $10 \mathrm{~Gb} / \mathrm{s}$ and $20 \mathrm{~Gb} / \mathrm{s}$ for the $1 \mathrm{~mm}$ and $0.5 \mathrm{~mm}$ long modulators, respectively. As expected, we were not able to measure error free operation $\left(\mathrm{BER}<10^{-9}\right)$ at higher bit rates, as a noticeable error floor caused by ISI arose around BER $10^{-5}$, which confirms the data rate limitation imposed by the modulator bandwidth. Furthermore, as a result of the 
lower ER, the shorter modulator exhibits a power penalty of $2.4 \mathrm{~dB}$ at $10 \mathrm{~Gb} / \mathrm{s}$, i.e. the overall transmitted optical power must be increased accordingly in order to maintain error free performance, relative to the longer one. An additional power penalty of $3 \mathrm{~dB}$ is also required as the data rate increases from $10 \mathrm{~Gb} / \mathrm{s}$ to $20 \mathrm{~Gb} / \mathrm{s}$ in the $0.5 \mathrm{~mm}$ long slow wave modulator.

\subsubsection{Conclusion}

Our experimental results demonstrate the potential for slow light propagation as a means to enhance the modulation efficiency of silicon electro-optical modulators. The main limitation arises from the insertion loss, which may in turn be minimized as a result of the much shorter achievable phase shifter lengths, even for moderate group indices. As a result of the enhanced modulation efficiency, error free modulation up to $20 \mathrm{~Gb} / \mathrm{s}$ has been demonstrated in a 500 $\mu \mathrm{m}$ long CMOS compatible slow wave modulator at a group index of only $\sim 11$. Furthermore, the modulator bandwidth could be significantly increased via optimization of the travelling wave electrodes for the slow light regime to ultimately improve the modulation speed to the levels that the $p n$ junction is intrinsically capable of achieving. Moreover, the perspective of using higher group indices as well as engineered slow light structures to achieve a constant group index with low losses in a broader wavelength range [94, 95, 129-131] also leaves room for further improvements. Overall, this result confirms the potential groundbreaking impact of slow light in the field of ultra fast and compact silicon modulators. 


\section{Chapter 4}

\section{Group Index Engineering in Silicon Slow Wave Structures}

This chapter is devoted to the design, characterization and fabrication of a novel type of engineered silicon slow wave waveguide exhibiting a high group index over a wide wavelength range. Potential applications of such a waveguide for wavelength-division-multiplexing (WDM) high speed modulation are explored.

\subsection{Motivation and Background}

In the previous chapter, we demonstrated the enhancement perspectives offered by the use of slow light in silicon modulators. We have seen that slow wave propagation may be readily achievable in high quality passive SOI dielectric structures such as line-defect photonic crystals (PhCs) [70, 91], coupled ring resonators [81], laterally corrugated waveguides [100, 132] to address a wide variety of applications such as optical buffering [81], non-linear enhancement in waveguides [133], small footprint switches [70, 71, 134], and electro-optical modulators $[43,122]$. However, one major issue is that this effect usually takes place at the edge of the photonic bands where dispersion due to local band curvature and losses due to fabrication disorder may be high [90, 105], which may remove in some cases the benefits of the slow light regime. In addition, the group index dependence upon frequency usually features a steep slope, limiting hence the operating optical bandwidth, that is to say the range of wavelengths or frequencies where the performance of a given device is maintained. However, we have demonstrated in the previous chapter that this slope may be smoothened via increasing the index contrast between the narrow and wide section of the LCWG. 
Our theoretical study showed the optical bandwidth of a "standard" LCWG is on the order of $\sim 1 \mathrm{~nm}$ for a group index of $\sim 10$ and drops to $\sim 0.2 \mathrm{~nm}$ for a group index of $\sim 20$ to maintain an DC ER "on target" of $10 \mathrm{~dB}$. These narrow group index dependent bandwidths make also the device very sensitive to temperature variations. Indeed, a $18^{\circ} \mathrm{C}$ variation is enough to produce a deviation in performance (here maintaining the DC ER on target of $10 \mathrm{~dB}$ ) for a group index of $\sim 10$, and only $2.5^{\circ} \mathrm{C}$ variation is tolerable when dealing with a group index of 20. Although, thermal management may be implemented to maintain the required level of performance, it may create added complexity and increase the overall power consumption. Therefore, to clearly overcome such concerns, the trail of engineered line-defect PhCs has been pursued with success. Indeed, high group indices in wide wavelength ranges with low loss [135] were achieved in these periodic waveguiding structures via different techniques [94, 95, 129]. However, PhCs exhibit generally large footprints. Here, we propose and demonstrate an alternative way to produce slow waves in low footprint engineered waveguides with a relatively high group index over a wide frequency range. The structures consist of conventional silicon LCWGs with holes patterned onto their wide section.

\subsection{Optical Design}

\subsubsection{Engineered slow wave structure overview}

As mentioned in the previous chapter, LCWGs typically feature a band gap between their fundamental and $1^{\text {st }}$ order odd modes with respect to their vertical plane of symmetry $X=0$ (TE-like mode). Here, we show that the addition of circular holes periodically patterned onto a LCWG (Figure 4-1) enables the dispersion relation to be tailored in order to obtain a nearly flat band, i.e. a region inside the Brillouin zone where the group index is constant over a determined frequency range. In what follows, we identify such band flattening process as a result of a combination of strong band gap widening and anticrossing effects [136]. 


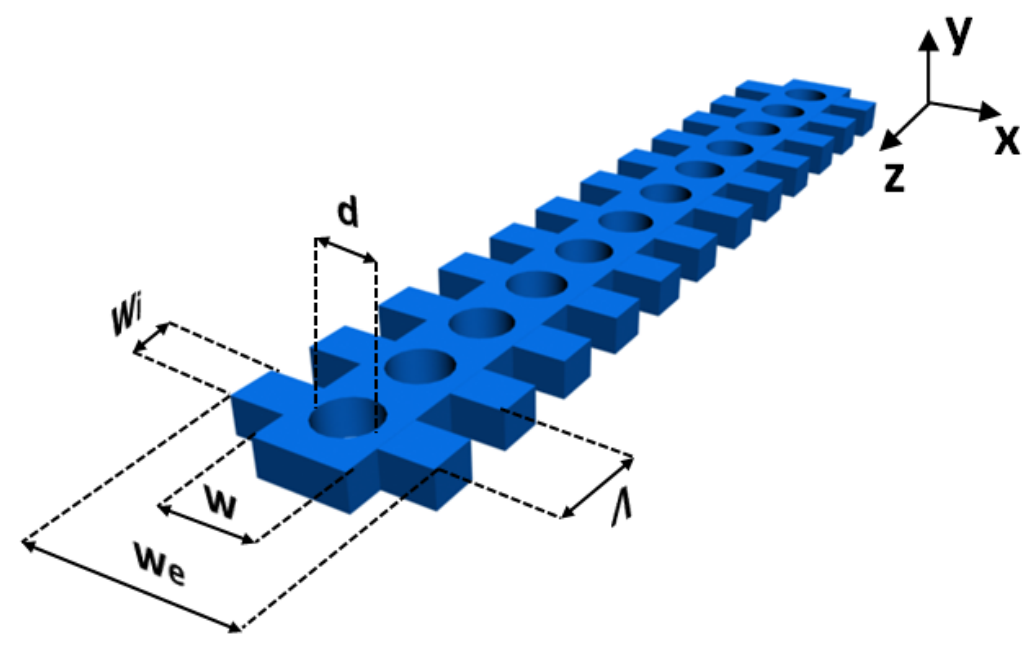

Figure 4-1: Schematic of the proposed engineered laterally corrugated waveguide

(ELCWG)

\subsubsection{Group index engineering guidelines}

In a conventional LCWG, the fundamental $(m=0)$ and $1^{\text {st }}$ order $(m=1)$ odd bands feature respectively positive and negative slopes towards the Brillouin zone edge where they both flatten (as shown in Figure 4-2), thus giving rise to slow wave propagation. By examining the mode profiles, one may observe that the fundamental mode amplitude is localized into the narrow section (Figure 4-2 (a)), while the $1^{\text {st }}$ order mode is confined into the wide section as illustrated in Figure 4-2 (b). Therefore, any alteration of the wide section of the dielectric waveguide is expected to produce a stronger effect on the $1^{\text {st }}$ order mode. Concretely, removing a certain amount of dielectric material causes the effective index to decrease due lower mode confinement and hence, photonic bands to be shifted towards higher frequencies. Here, we altered the LCWG unit cell by introducing a circular hole of diameter $d$ into its wide section, as depicted in Figure 4-1. As a result of this low refractive index hole patterning, the bands are shifted towards higher frequencies accordingly with however, noticeable differences at the Brillouin zone edge due to the stronger perturbation experienced by the $1^{\text {st }}$ order mode with respect to the fundamental mode. A close look at the mode profiles after hole patterning shown in Fig.4-2 (c)-(d), corroborates the latter results. On the other hand, for lower wave vectors, the $2^{\text {nd }}$ order mode $(m=2)$ interacts with the $1^{\text {st }}$ order mode just beyond the light line, which gives rise to an anticrossing. As low refractive index holes are introduced into the LCWG, this anticrossing point is displaced both in frequency and wave vector. Furthermore, due to stronger coupling between $1^{\text {st }}$ and $2^{\text {nd }}$ order modes, it widens and thus pushes the $1^{\text {st }}$ order band downwards as 
can be seen in Fig. 4.2 Eventually, the $1^{\text {st }}$ band flattening occurring between the anticrossing point and the Brillouin zone edge is attributed to the combination of both abovementioned effects. From an engineering point of view, our PWE simulation results show that the $1^{\text {st }}$ order band flatness can be chosen by simply varying the hole diameter.

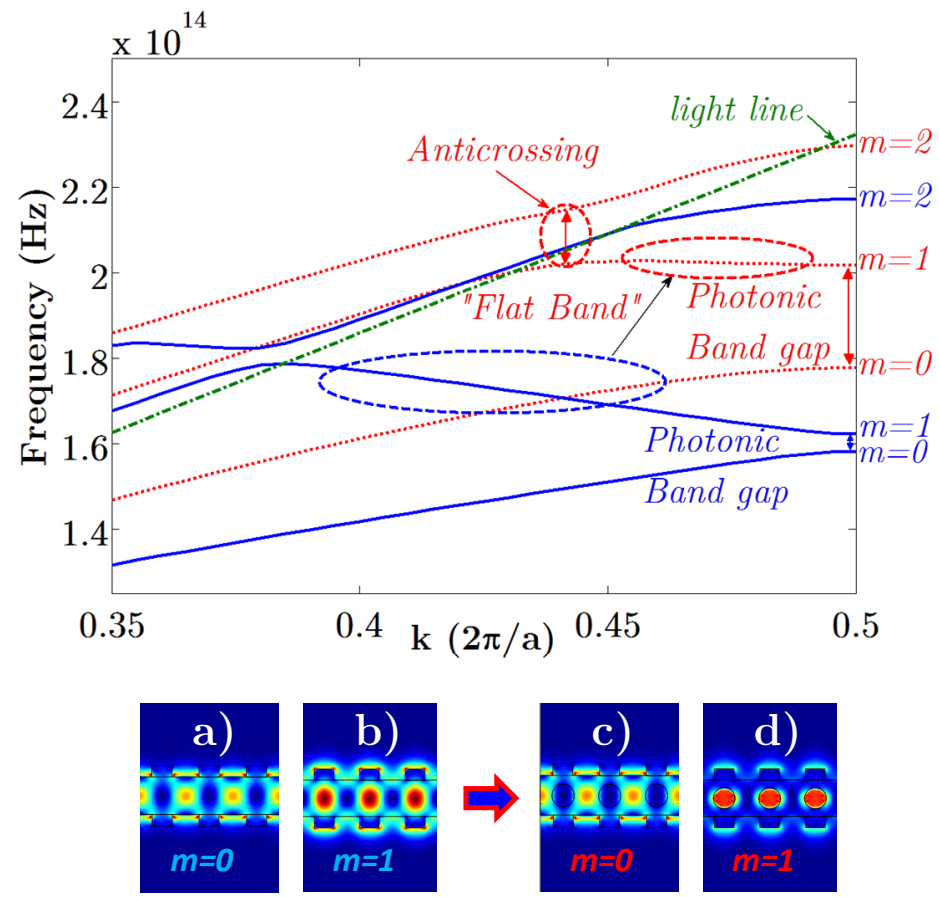

Figure 4-2: Band "flattening" process: Blue solid lines and red dashed lines stands for the first three bands before and after hole patterning, respectively. a) and b) describe the unperturbed mode profiles at the band edge while c) and d) depict the altered mode profile after hole patterning.

\subsubsection{Modeling of the engineered laterally corrugated waveguides}

Following the previously mentioned guidelines, we simulated numerically the engineered waveguides with a commercial 3D PWE simulator [74]. For completeness, we indicate hereafter the theoretical parameters that were chosen to center the flat band section around $1550 \mathrm{~nm}$ wavelength with a corresponding group index value of $\sim 13.5: W=400 \mathrm{~nm}, W_{e}=600 \mathrm{~nm}, W_{i}=220 \mathrm{~nm}, d=230 \mathrm{~nm}$ (to be varied), $\Lambda=460 \mathrm{~nm}$ and $H=220 \mathrm{~nm}$. The silicon refractive index is $n_{S i}=3.47$ and the optical waveguide is covered with silicon dioxide with $n_{S_{i O} 2}=1.444$. From Figure 4-3 (a), it can be seen that the $1^{\text {st }}$ order dispersion band becomes flatter as the hole diameter is enlarged, thus causing the group index to increase. Then, as the dispersion relation features a nearly constant slope over a relatively wide 
frequency range, this engineered group index remains nearly constant over the latter, as depicted in Figure 4-3 (b). Besides, two useful observations can be made:

First, as the effective index decreases because we remove dielectric material, the constant group index region is blue shifted. To compensate for such displacement, one may adjust the position the flat band in the desired wavelength range by simply altering the grating period.

(a)

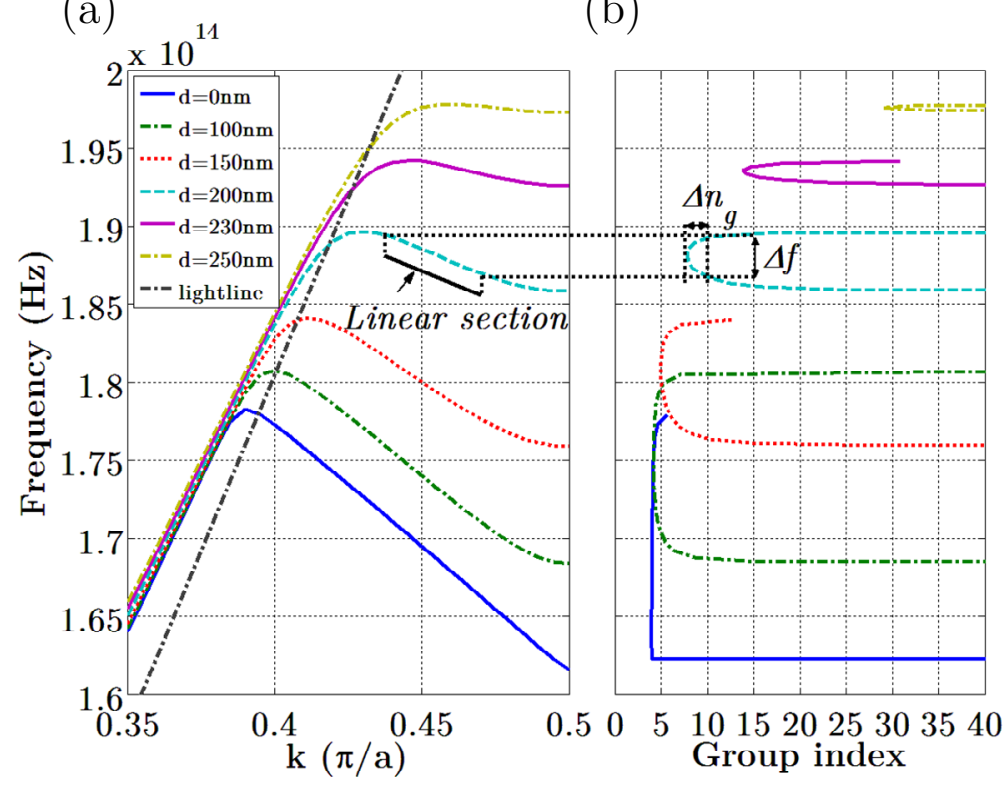

Figure 4-3: a) Band flattening dependence against hole diameter. b) Group index versus wavelength for varying hole diameter.

Second, it should be noticed that although the group index increases as the hole diameter does, the bandwidth of the flat band decreases, setting therefore a tradeoff between high group index and large bandwidth. To be consistent with previous works, we define the bandwidth as the frequency (resp. wavelength) range where group index variations of $\pm 10 \%$ are tolerated [94]. Figure 4-4 depicts on the same graph the average group index and associated bandwidth dependence on hole size. The average group index is not significantly altered for hole diameters lower than $d=150 \mathrm{~nm}$ with a value maximum value of $n_{g}=5.5$ while the bandwidth is significantly reduced from $\sim 160 \mathrm{~nm}$ to $\sim 50 \mathrm{~nm}$. For hole diameters higher than $d=150 \mathrm{~nm}$ the group index experiences a strong increase while the bandwidth seems to follow a nearly monotonous tendency. This suggests that to significantly engineer the group index in such a waveguide, the hole diameter should be at least $d=200 \mathrm{~nm}$ with the fabrication constraints in mind. 


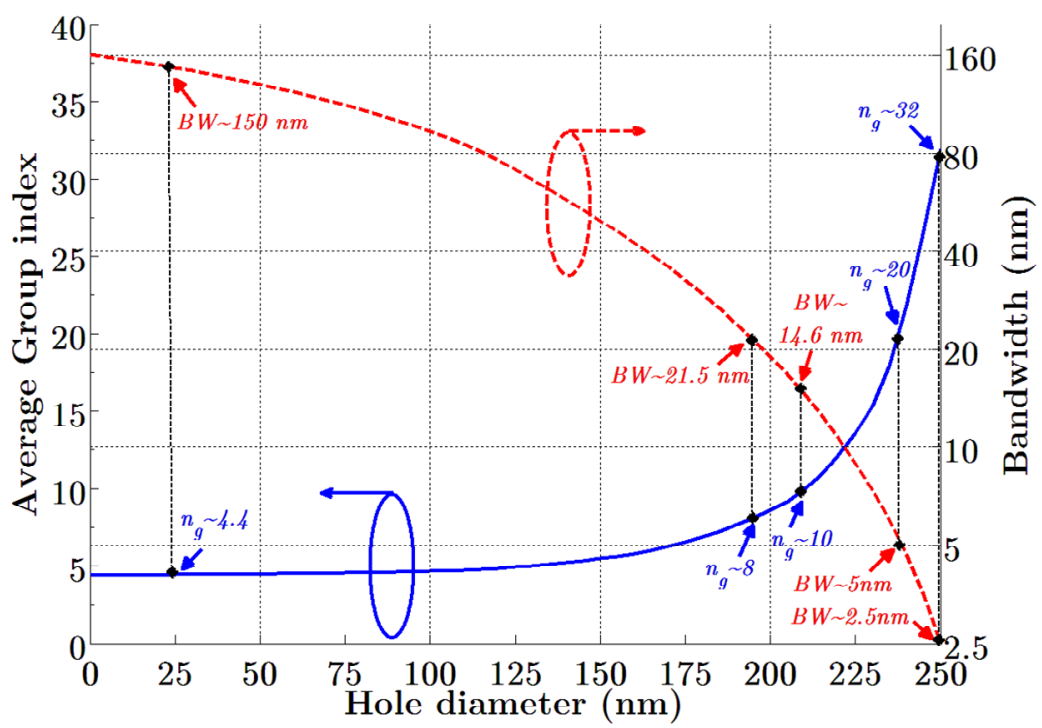

Figure 4-4: Average group index (blue solid line) and bandwidth (red dashed line) against hole diameter.

\subsection{Electrical Design}

\subsubsection{DC analysis of a slow wave phase shifter with broad optical}

\section{bandwidth}

Achieving high group index values over a broad bandwidth is extremely interesting as it removes simultaneously the issues arising from group velocity dispersion (essentially pulse broadening) and the limited optical bandwidth of the slow wave structure. Both are generally caused by the steep group index dependence upon wavelength. According to Figure 4-4, the operating wavelength bandwidth of our ELCWG is greatly increased compared to the LCWG. Assuming a $\pm 10 \%$ variation (see section 3.4 .4 ), and for a group index of $\sim 10$, the optical bandwidth is $\sim 14.6 \mathrm{~nm}$, which is much higher than the $1 \mathrm{~nm}$ value achieved in a LCWG for a similar group index. This enlarged operating wavelength bandwidth provides not only relaxed tolerance on temperature variations, but also opens the way to wavelength division multiplexing (WDM) based on a array of slow wave EOMs consisting of ELCWGs. Additionally, this dispersion-free waveguide allow pulses to propagate without broadening, which might be particularly interesting for delay lines and all-optical applications. This will be discussed further on.

The particular shape of the ELCWGs and the fact that the slow mode concentrates the majority of its power within the low refractive index region (i.e. 
the hole) may however impair the modulation efficiency of the slow wave EOM. This is due to the fact that charge carrier motion (and hence refractive index modulation) takes place within the silicon and overlaps only with the fraction of the optical mode that propagates throughout this material. Therefore, in theory, the ELCWG-based modulator is expected to be less efficient than that based on conventional LCWGs.

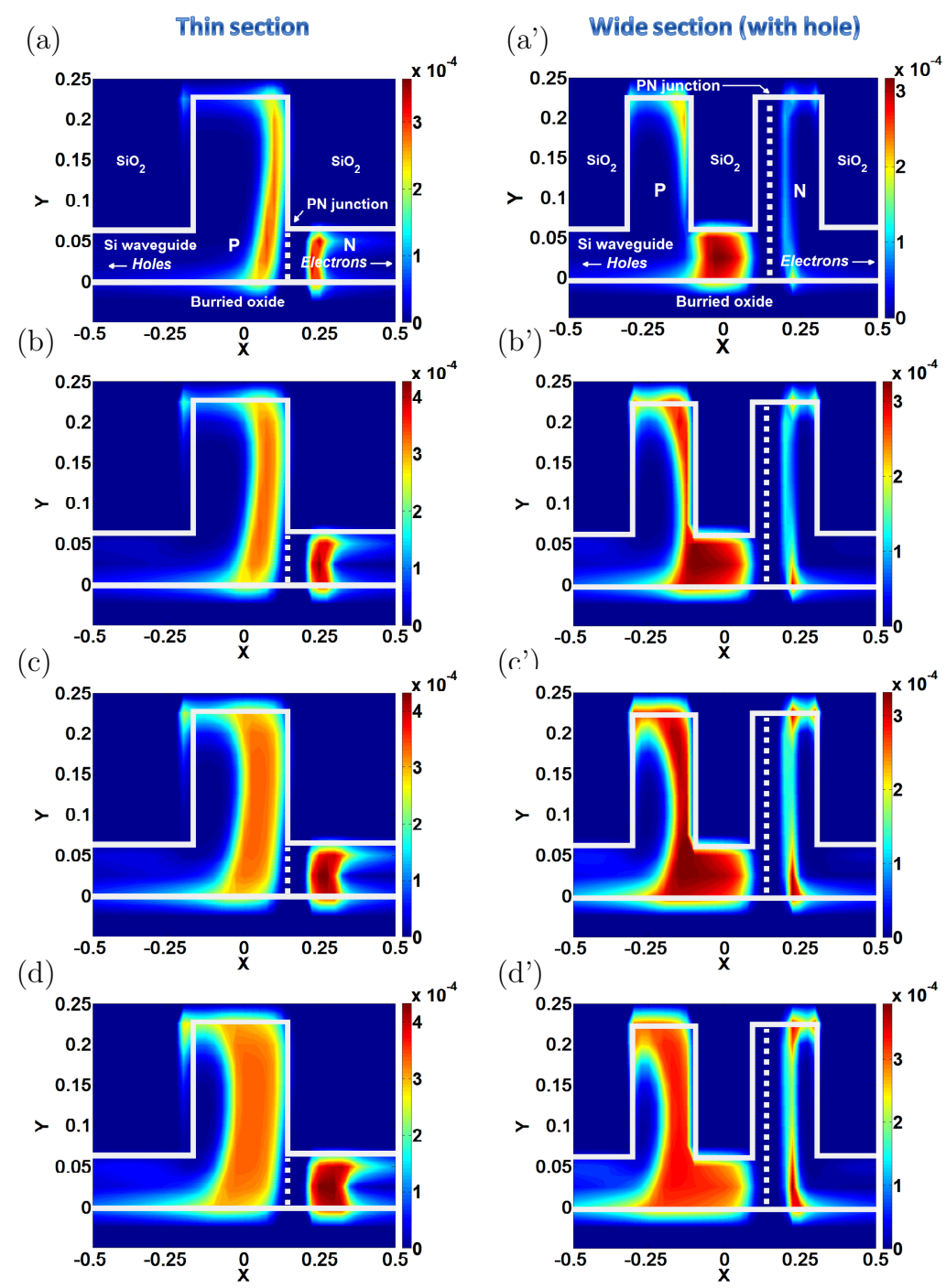

Figure 4-5: real refractive index change $\Delta n$ under different applied drive voltages (a), (a') $\Delta V=-2 \mathrm{~V}$; (b), (b') $\Delta V=-4 \mathrm{~V}$; (c), (c') $\Delta V=-6 \mathrm{~V}$; (d), (d') $\Delta V=-8 \mathrm{~V}$; for the thin and wide sections of the $1 \mathrm{D}$ ELCWG, respectively. The junction is in steady state. Waveguide cross section boundaries are represented by the white plain lines

In order to investigate how efficient an ELCWG-based modulator would be, a rather simple analysis has been carried out. However, because our electric device simulator is only 2 dimensional, we are here assuming that the holes are square in the PWE analysis. As square holes occupies more volume than cylinders 
(the latter is the actual shape of the holes patterned onto the LCWG), the volume of the active silicon region overlapping with the optical mode will be smaller. This leads us to underestimate the theoretical efficiency of the device.

Figure 4-5 illustrates the real refractive index change inside the ELCWG for varying reverse bias voltage. We are assuming here that the modulator is based on the fabrication process described in section 3.6.2 [49], i.e. where the $p n$ junction is self aligned with the LCWG narrow section edge. As observed, the refractive index change takes places within the silicon, therefore, following the simulation procedure described in section 3.4.2, we can calculate the effective index change in the ELCWG via the PWE method and contrast its modulation efficiency with that of a $400 \mathrm{~nm}$ rib waveguide embedded in the same $p n$ junction as well as that of the conventional LCWG as respectively illustrated in Figure 4-6 (a) and (b). We may first observe that both slow wave waveguides are more efficient than the $400 \mathrm{~nm}$ wide rib waveguide modulator owing to the moderately high group index.

We remind that the enhancement factor (see section 3.7.6) gives an estimate on how much the length or voltage (or a combination of both) may be reduced compared to $400 \mathrm{~nm}$ wide rib WG modulator. As can be seen the enhancement factor reaches 3 for a group index of $\sim 13$, which means that the engineered SW EOM length or drive voltage may be reduced by a factor 3 . Furthermore, one may observe in Figure 4-6 b) that the modulation efficiency of the ELCWG relative to that of the LCWG is highly dependent upon the group index. First, a strong decrease in efficiency takes places owing the fact that even a small hole affects the intensity of the overlap between the optical mode and the free carriers. However, as the group index starts to increase (as the hole diameter does), let us say from $n_{\mathrm{g}} \sim 7$, the curve trend is reversed because the enhanced slow light interaction with free carriers compensates for the smaller modulating area, leading ultimately to a $75 \%$ relative efficiency for a group index of $\sim 13$. These results confirm the potential of our ELCWG for broad band and enhanced modulation applications. 
(a)

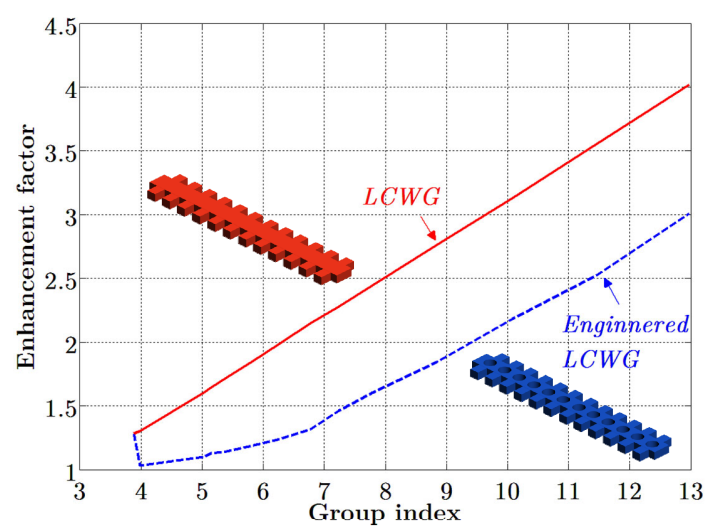

(b)

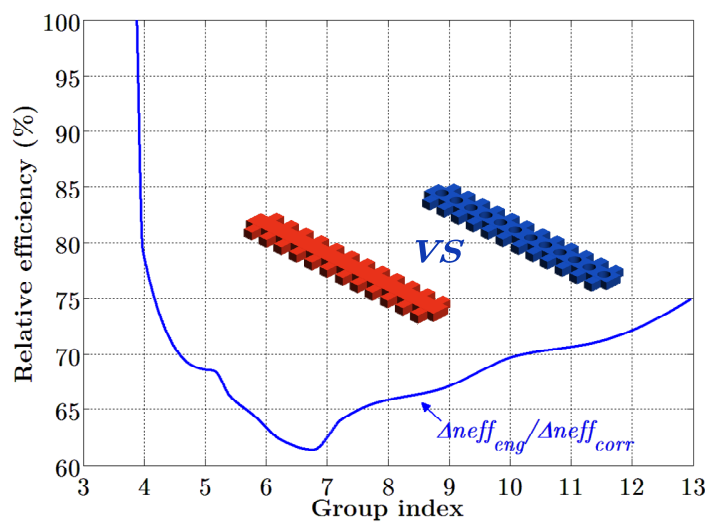

Figure 4-6: (a) Enhancement factor compared to a $400 \mathrm{~nm}$ rib waveguide and (b) relative modulation efficiency of the ELCWG compared to the LCWG.

\section{Amplitude modulation}

Just as for the conventional LCWG EOM, amplitude modulation in the DC regime is performed via the integration of the ELCWG in a MZI. To fully appreciate the bandwidth enhancement, we have simulated a symmetric MZI with engineered SWPS of length $1430 \mu \mathrm{m}$ placed in each arm. The transmission of such a symmetric MZI is given by the following Equation.

$$
\begin{aligned}
T & =\cos ^{2}\left(\frac{\pi}{\lambda} L_{S W P S}\left(n e f f_{S W P S, u p}\left(V_{u p}\right)-\operatorname{neff}_{S W P S, \text { down }}\left(V_{\text {down }}\right)\right)\right) \\
& =\cos ^{2}\left(\frac{\pi}{\lambda} L_{S W P S}(\Delta n e f f(V))\right)
\end{aligned}
$$

Where neff $f_{S W P S, \text { up }}$ (resp. neff $f_{S W P S, \text { down }}$ ) is the modal effective index in the upper (resp. lower) arm. Here, for the sake of simplicity, we only actuate on the lower arm, which means that the modulator is in a single drive regime. In other words the upper arm remains unbiased $\left(V_{\text {down }}=0 \mathrm{~V}\right)$. Nevertheless, push-pull operation may also be an advantageous option in practice as it enables the drive voltage to be decreased by a factor of two and also helps reduce chirp induced by the modulator. Figure 4-7 shows the results of a SW EOM single drive operation based on ELCWGs. 

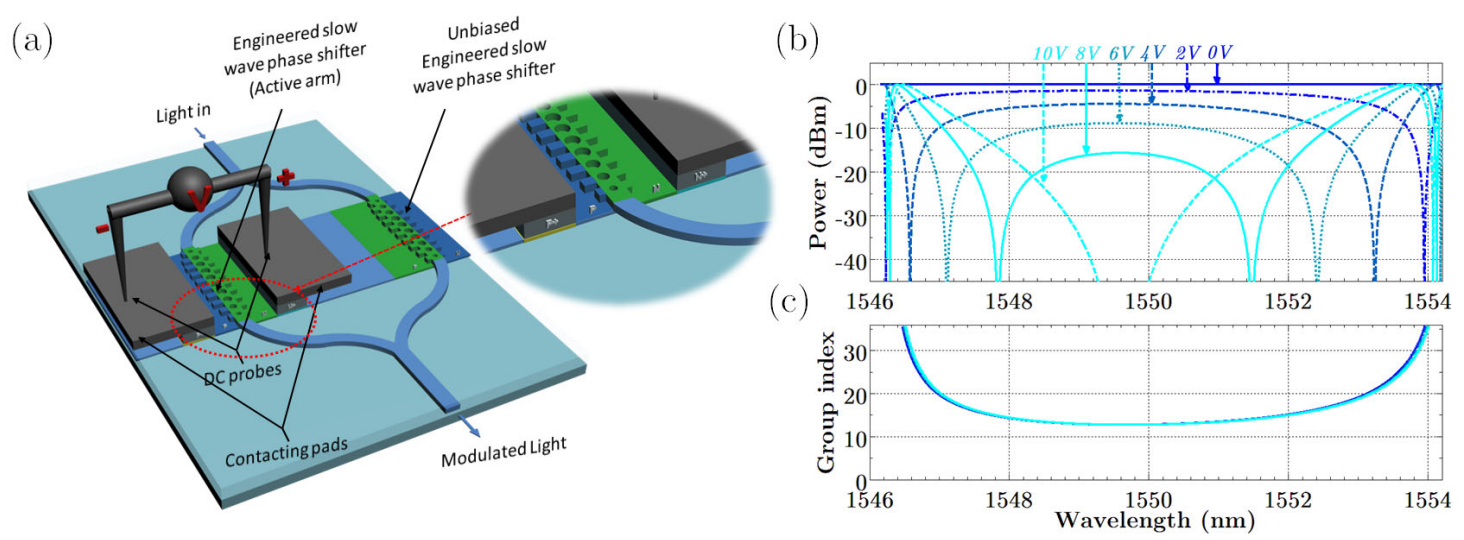

Figure 4-7: (a) Schematic of the symmetric MZM based on ELCWG (b) Transmission spectrum for varying applied reverse bias (from $V=0 \mathrm{~V}$ (unbiased) to $V=-10 \mathrm{~V}$ ) of a MZM with a $1430 \mu \mathrm{m}$-long engineered SWPS inserted into both active arms (c) Group index dependence upon wavelength for varying applied reverse bias (from $V=0 \mathrm{~V}$ (unbiased) to $V=-10 \mathrm{~V}$ )

As can be observed, the useful optical bandwidth is significantly enhanced compared to the conventional LCWG-based modulator. Indeed, for this specific modulator, the achieved DC extinction ratio is higher than $10 \mathrm{~dB}$ over a bandwidth of $6.5 \mathrm{~nm}$ and for a $6 \mathrm{~V}$ reverse bias. The minimum group index value over this bandwidth is approximately $\sim 13$ with a relative predicted modulation efficiency reaching $75 \%$ of that of the LCWG. This improved performance may find applications in WDM systems with aggregate data rate transmission reaching $100 \mathrm{Gbit} / \mathrm{s}$. Indeed, we may envision the possibility of integrating 4 engineered SW EOMs integrated with $4 \mathrm{CW}$ lasers emitting at different wavelengths (or one single laser emitting multiple wavelengths) separated by $200 \mathrm{GHz}(\sim 1.6 \mathrm{~nm})$ to ensure low crosstalk between channels. Such a standard International Telecommunication Union (ITU) grid spacing have been readily achieved in SOI technology either based on array waveguide gratings (AWGs) or cascaded ring resonators (CRRs) [137, 138]. Figure 4-8 below illustrates a concept schematic of how a 4 x 25 Gbits/s WDM system could look like. The 4 different wavelengths with $200 \mathrm{GHz}(1.6 \mathrm{~nm})$ channel spacing are injected into each of the 4 ELCWGbased MZI amplitude modulators and multiplexed in a rib waveguides via 4 cascaded ring resonators with slightly different radii to provide resonances centered respectively at the 4 input wavelengths. To compensate for probable fabrication deviation, thermal (or electrical) tuning of the 4 individual ring resonators may be required. For clarity the thermal tuning elements have not been represented. 


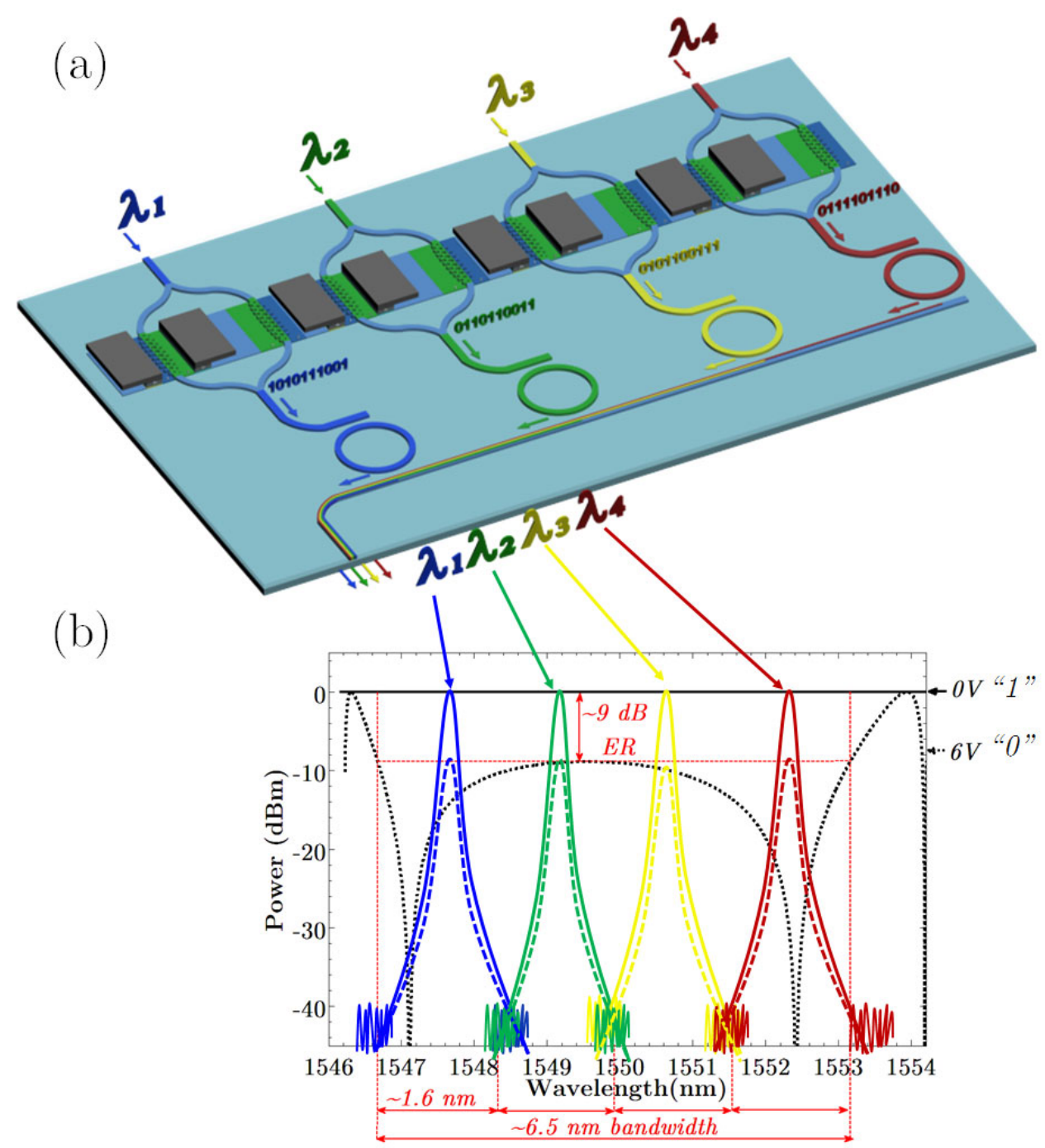

Figure 4-8: (a) Concept schematic of a 1x4 ring resonator-based WDM system with aggregate data rate capabilities of $100 \mathrm{Gbit} / \mathrm{s}$ (4x25 Gbits/s). 4 different wavelengths with $200 \mathrm{GHz}(1.6 \mathrm{~nm})$ channel spacing are injected into each of the 4 ELCWG-based MZI amplitude modulators and multiplexed in a rib waveguide via 4 cascaded ring resonators with slightly different radii to provide resonances centered respectively at the 4 input wavelengths. The 4 modulated signals are then photodetected at the chip output. (b) The blue plain and dashed lines illustrate respectively the DC "on" (' 1 ) "off" ('0') symmetric slow wave MZI's spectra between $0 \mathrm{~V}$ and $6 \mathrm{~V}$, together with the chip output spectra consisting of the 4 modulated channels.

In conclusion, we have shown the potential of ELCWG for providing sufficient useful optical bandwidth to relax tolerances on fabrication deviations and thermal sensitivity at the expense of slightly lower modulation efficiencies compared the conventional LCWG. Additionally, we have shown that ELCWG provide enough optical bandwidth to be implemented in an integrated 1x4 WDM transmission system to potentially achieve an aggregate data rate of $100 \mathrm{Gbits} / \mathrm{s}$. 
Experimental evidence of error free 20 Gbits/s modulation in LCWG and modulation rate capabilities of up $40 \mathrm{Gbits} / \mathrm{s}$, suggest that such a high speed transmitter may be achievable in a foreseeable future.

\subsection{Experimental results}

\subsubsection{Passive characterization results}

In order to provide experimental evidence of group index engineering, the fabrication of this particular structure was carried out on a $220 \mathrm{~nm}$ thick SOI wafer. The optical waveguides were patterned by electron beam lithography on FOX 12 negative resist, deep-etched by reactive ion etching (RIE) and covered with a roughly $700 \mathrm{~nm}$ thick silicon dioxide layer. A SEM image of the fabricated structure is shown in below.

(a)

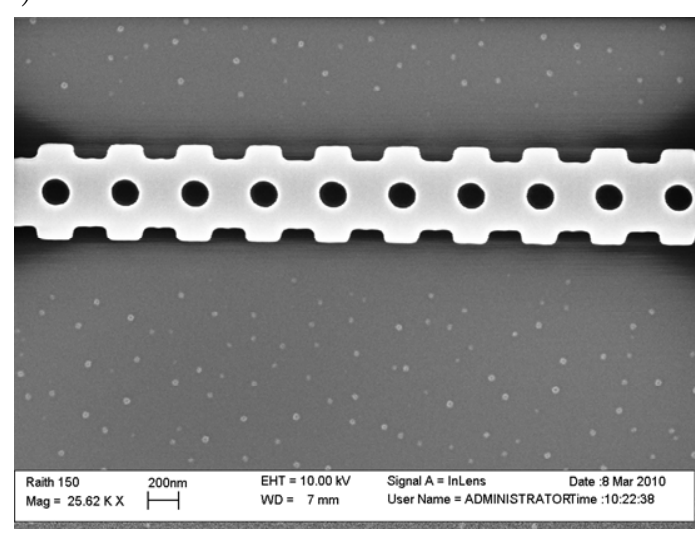

(b)

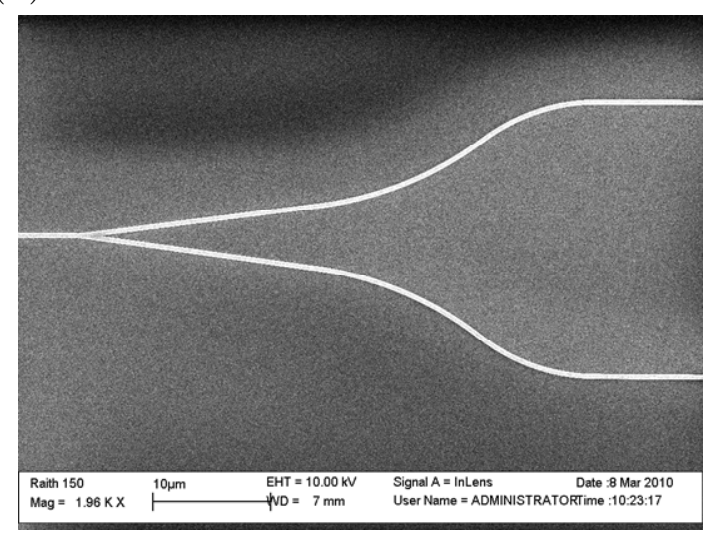

Figure 4-9: SEM images of the fabricated structures (a) ELCWG (b) MZI Y-junction splitter

The optical characterization was carried out using a tunable laser, whose output near-infrared light was butt-coupled to the optical structure through a lensed fiber and finally detected in free space by an infrared detector.

The transmission of a $100 \mu \mathrm{m}$ long corrugated waveguide is shown in Figure 4-10 (a). The waveguide transmission window corresponds to the "flat portion" of the $1^{\text {st }}$ order mode. It should be highlighted, that the spectrum is red shifted with respect to our theoretical simulations, certainly due to corrugated waveguide section widening. The FP ripples arise from the lack of efficient tapers required to minimize the impedance mismatch between the slow and fast modes. Interestingly, the transmission window is wider than that theoretically simulated with the PWE method. We believe that this is likely due to the fact that PWE 
simulations assume infinitely long waveguides, which is obviously not the case in practice. An infinitely long ELCWG may be thought as an optical filter with sharp edges while a finite-length ELCWG exhibits smoother ones. To give some qualitative evidence, 3D FDTD simulations have been performed for 4 different ELCWG lengths $(5,10,20$ and $30 \mu \mathrm{m})$. The transmitted spectra are depicted in Figure 4-11. As expected the transmission window edges become sharper for increasing waveguide length.

The group index has been determined by an on-chip interferometric method consisting in inserting a $50 \mu \mathrm{m}$ long corrugated waveguide in one arm of a integrated Mach-Zehnder interferometer (MZI) consisting of two low loss Y junctions (see Figure 4-9 (b)) to efficiently split and recombine light. The reference arm, consisting of a $400 \mathrm{~nm}$ wide waveguide, features a constant group index $n_{g, r e f} \sim .2$ over the considered spectral range. This group index value has been calculated in order fit the actual waveguide width, which was $450 \mathrm{~nm}$. The extraction of the group index from the resulting interferometric spectrum is obtained from the following Equation:

$$
n_{g}(\lambda)=\frac{\lambda_{\min } \lambda_{\max }}{2 L \Delta \lambda}+n_{g, r e f}
$$

where $L$ is the MZI length and $\lambda_{\min }$ and $\lambda_{\max }$ are the respective positions of the minima and maxima of transmission [70]. Our experimental results illustrated in Fig. 5, show that an average group index value of approximately $n_{g} \sim 13.5$ has been measured over a $\sim 14 \mathrm{~nm}$ wide wavelength range in a $50 \mu \mathrm{m}$ long waveguide. Propagation losses around $18 \mathrm{~dB} / \mathrm{mm}$ at such group index were estimated in straight corrugated waveguides by means of the cut-back method. These rather high losses are essentially due to fabrication imperfections as well out-of plane scattering due to the lack of slow mode confinement in the vertical directions. (just as in line defect 2D PhCs). 

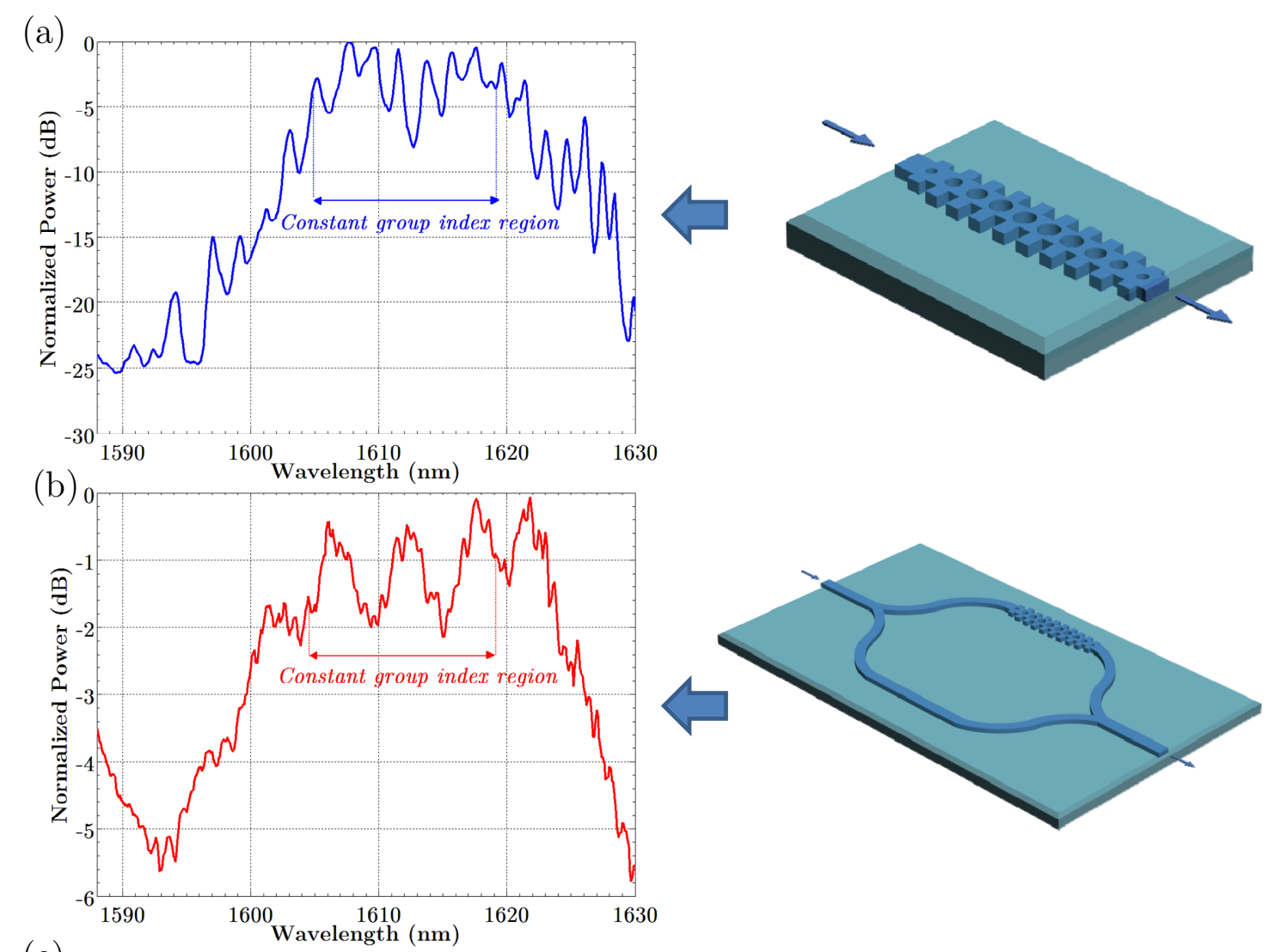

(c) 50

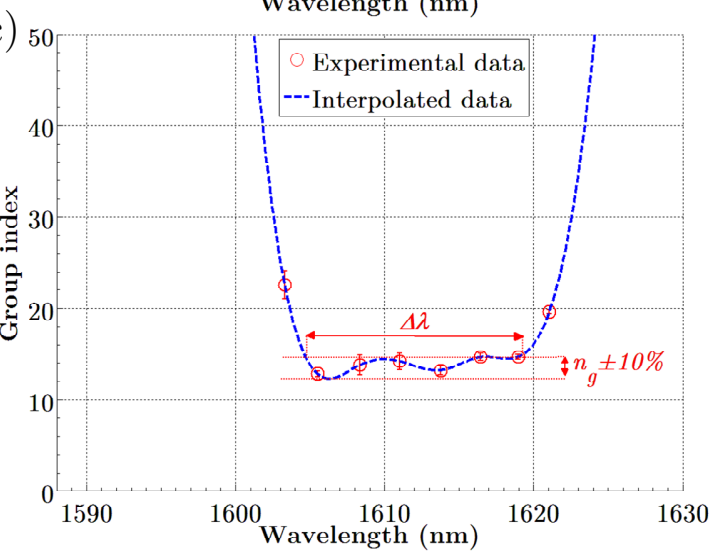

Figure 4-10: (a) Normalized transmission spectrum of a $100 \mu \mathrm{m}$ long ELCWG. (b) Normalized transmission spectrum of a $500 \mu \mathrm{m}$ long MZI loaded with a $50 \mu \mathrm{m}$ long ELCWG. (c) Experimental group index dependence against wavelength. Insets show schematics of the integrated structures together with their corresponding transmission spectra. 


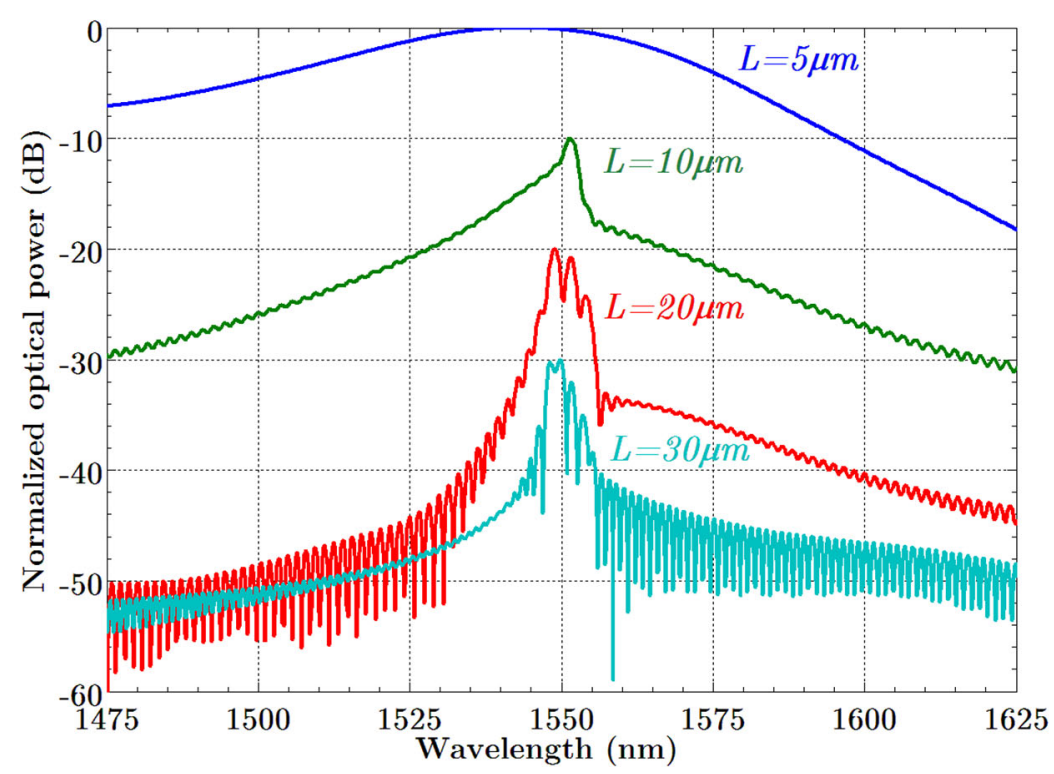

Figure 4-11: 3D FDTD simulation results of 4 ELCWGs with 4 different waveguides of lengths 5, 10, 20 and $30 \mu \mathrm{m}$. Spectra are normalized and shifted by $10 \mathrm{~dB}$ for clarity.

\subsubsection{Efficient coupling to the slow light mode}

As seen from the experimental results, FP power ripples are a clear indication that the coupling from the fast to slow light regime is not optimum. To overcome this issue efficient tapers should be designed. No experimental results have provided sufficient accuracy to clearly demonstrate the improvement that such coupling structures may bring over non-tapered ELWGs. However our 3D FDTD results depicted in Figure 4-12 suggest that assuming a rather simple taper consisting of four elements the transmission is clearly enhanced and FP ripples are significantly smoothened. These simulations are only preliminary steps towards optimized tapers for such a slow wave waveguide. Further optimization and testing should be performed. However, because the dimensions of the first taper elements are small to match as good as possible that of the rib waveguide, the critical dimensions are hence very small $(25 \mathrm{~nm})$. While this should not be a issue for e-beam lithographic process, it might however give rise to minimum resolution issues in a standard CMOS $198 \mathrm{~nm}$ deep-UV lithography process, which accept critical dimensions of $\sim 50 \mathrm{~nm}$. Consequently, optimized tapers are readily achievable theoretically but may be designed for a given lithographic process with its own design rules. 

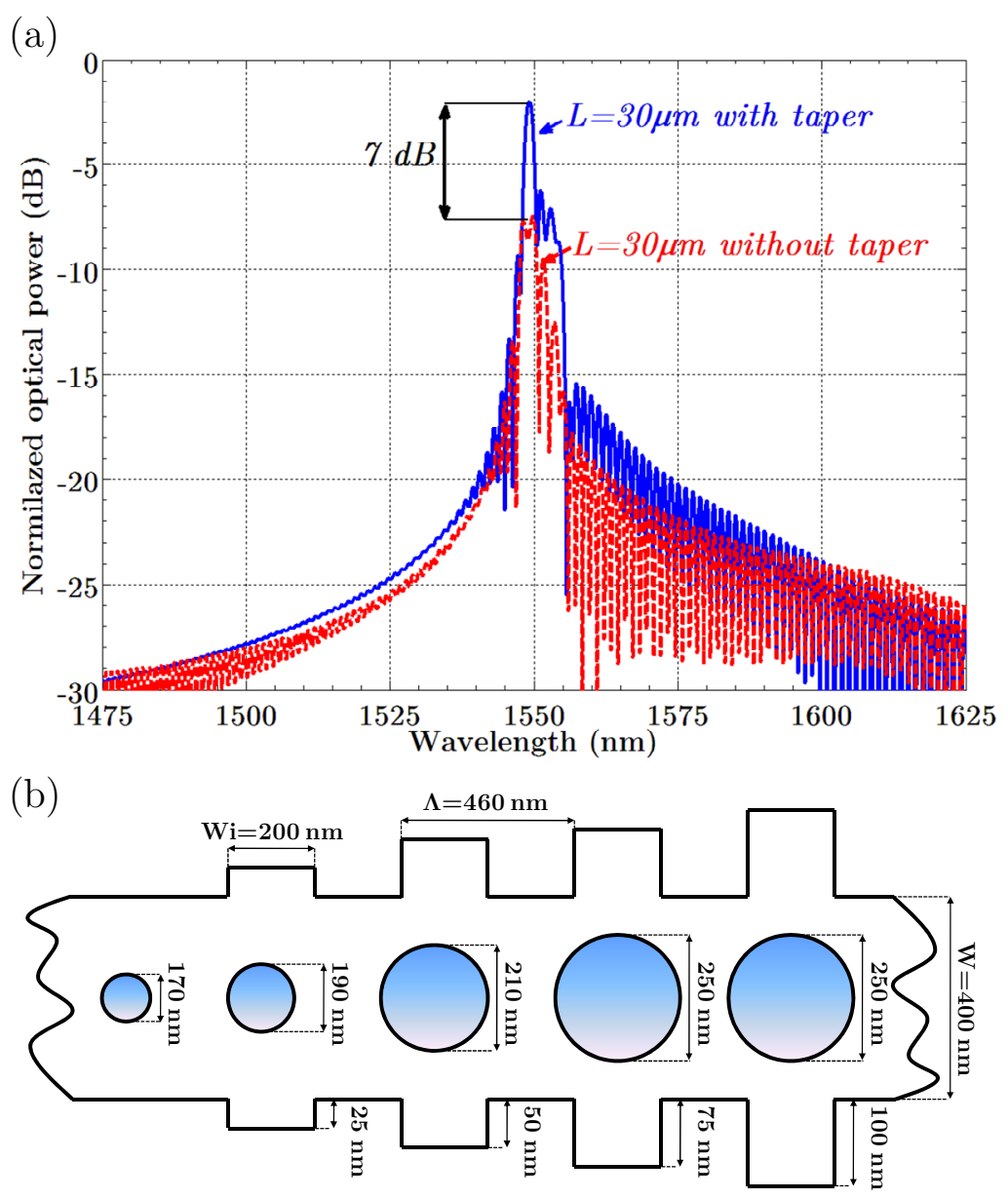

Figure 4-12: (a) 3D FDTD simulation results of tapered and non-tapered LCWGs (b) Taper dimensions.

\subsubsection{Conclusion}

In conclusion, we have demonstrated a novel of type group index engineered 1D slow wave waveguide that solves the issue of the usual band edge operation of conventional LCWGs and slow wave structures exhibiting photonic band gaps. Indeed, nearly constant group index in a relatively broad wavelength range is readily achievable by patterning circular holes onto the wide section of a LCWG. Our experimental results show that such a novel engineered waveguide features an average group index $n_{g} \sim 13.5$ over a $\sim 14 \mathrm{~nm}$ wide wavelength range. Higher group indices at the expense of lower bandwidth and higher propagation losses are theoretically feasible via increasing the hole diameter. Key aspects such as efficient coupling to the slow light regime as well as sidewall roughness reduction have to be improved for the next generation of waveguides. Overall, this type of slow light structure might open the way to numerous applications 
such as high speed WDM transmission systems for high performance parallel computing owing to its enhanced optical bandwidth, compactness and ease of fabrication compared to line-defect 2D PhCs. 


\section{Chapter 5}

\section{Conclusions and Prospects}

In conclusion, this work has provided significant advances in the field of silicon photonic devices via demonstrating how the use of slow light, that is, when light travels significantly slower than in air or in a vacuum, allows for the realization of enhanced silicon modulators operating at up to 40 Gbits/s. It has been shown that the electro-optical bandwidth of the device is impaired only by the velocity mismatch between the electrical and optical signals and may be improved up to the speed the self aligned $p n$ junction is intrinsically capable of achieving via convenient TW electrode design. Additionally, decreasing the driving voltage down to the $45 \mathrm{~nm}$ node CMOS electronic technology requirements is the next milestone to be achieved for slow wave modulators.

Meanwhile, further improvements in terms of operating optical bandwidth in order to sustain a similar level of performance over a broader wavelength range are under investigation. We have indeed demonstrated that one dimensional slow light structures, which usually exhibit slow light operation over a limited optical bandwidth may readily be engineered to achieve broader operating bandwidth. This implicates relaxed fabrication tolerances and increases the reliability of one dimensional slow light structures. Further research efforts should be focused on generalizing the engineering recipe and lower the propagation loss through an optimized fabrication process to eventually achieve the desired level of performance. Potential applications such as WDM modulation, enhanced biological sensing, optical buffering, and enhanced non-linear devices, are foreseen. Passive engineered slow light structures and modulators based on this particular broad band slow light waveguide are also under investigation. Improvements in terms of optimized tapering sections for both types of one dimensional slow light 
waveguides (LCWGs and ELCWGs) are expected to dramatically enhance the coupling to the slow light mode and hence reduce further the insertion loss figure.

Additionally, this work gathered some fundamental aspects and underlying physical mechanisms of silicon-based modulators, in order to make it available as a starting point for future investigations and engineering studies not only in the specialized field of silicon modulators but also for all sorts of active silicon photonic devices based on the plasma dispersion effect.

Overall, we believe that the results presented in this work may have both scientific and commercial importance because the combination of efficiency and compactness has been at the heart of the research strategy within the field of silicon electronics for more than half a century. This approach is sought similarly in the emerging field of silicon photonics although in a different framework than that ruled by Moore's law. Harnessing light-matter interactions in order to push forward tirelessly the limits of photonic devices is and will remain a central objective of the community of photonic scientists and engineers. This work humbly takes this research field advancement one step further by contributing to the realization of highly efficient and ultra compact CMOS compatible silicon photonic modulators via enhanced light-matter interaction. 


\section{Appendix A}

\section{A.1 ATLAS code example}

The following code simulates the static behavior between 0 and $-10 \mathrm{~V}$ (with $1 \mathrm{~V}$ increments) of an asymmetric pn junction in formed in a $300 \mathrm{~nm}$ silicon waveguide.

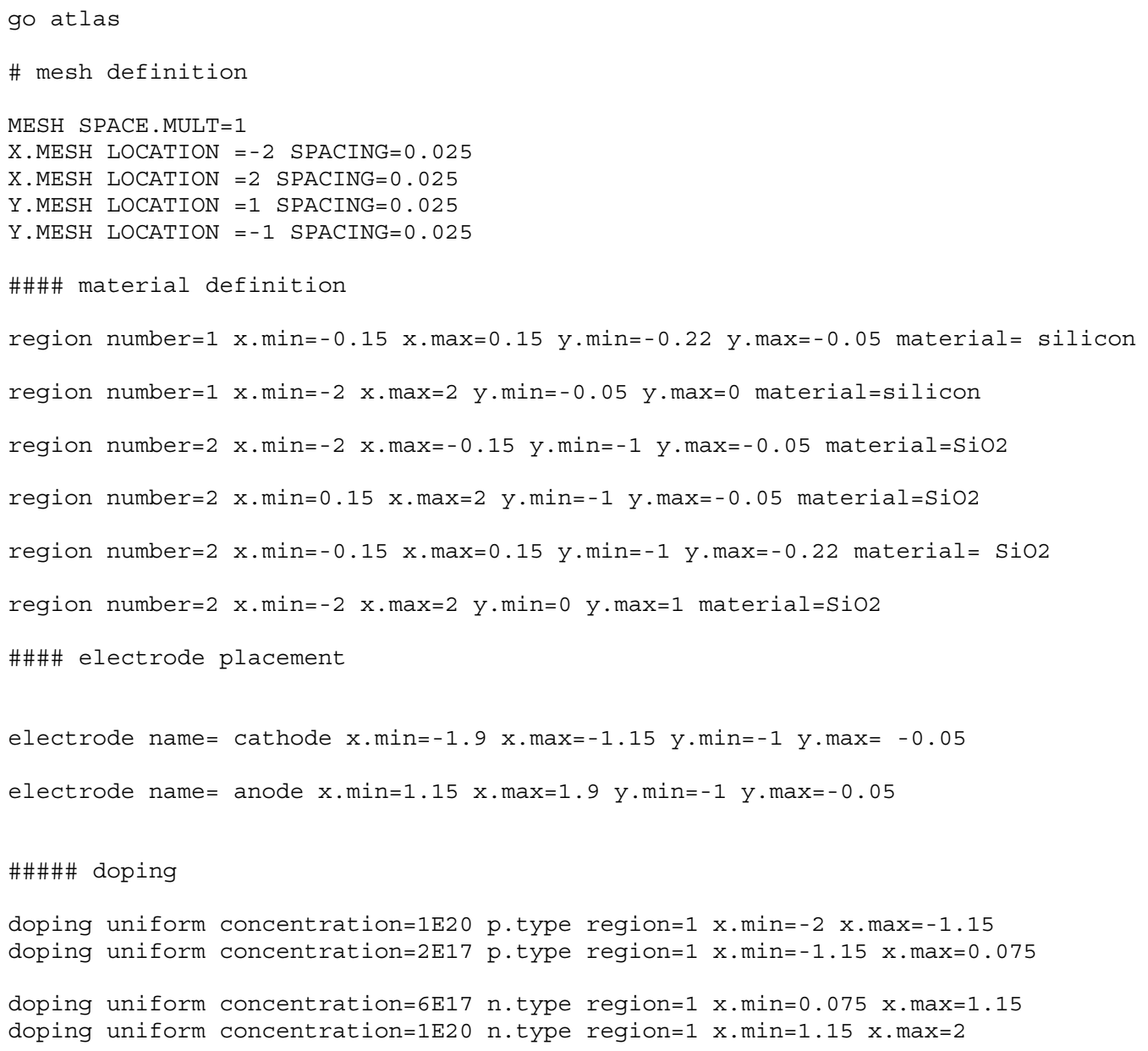




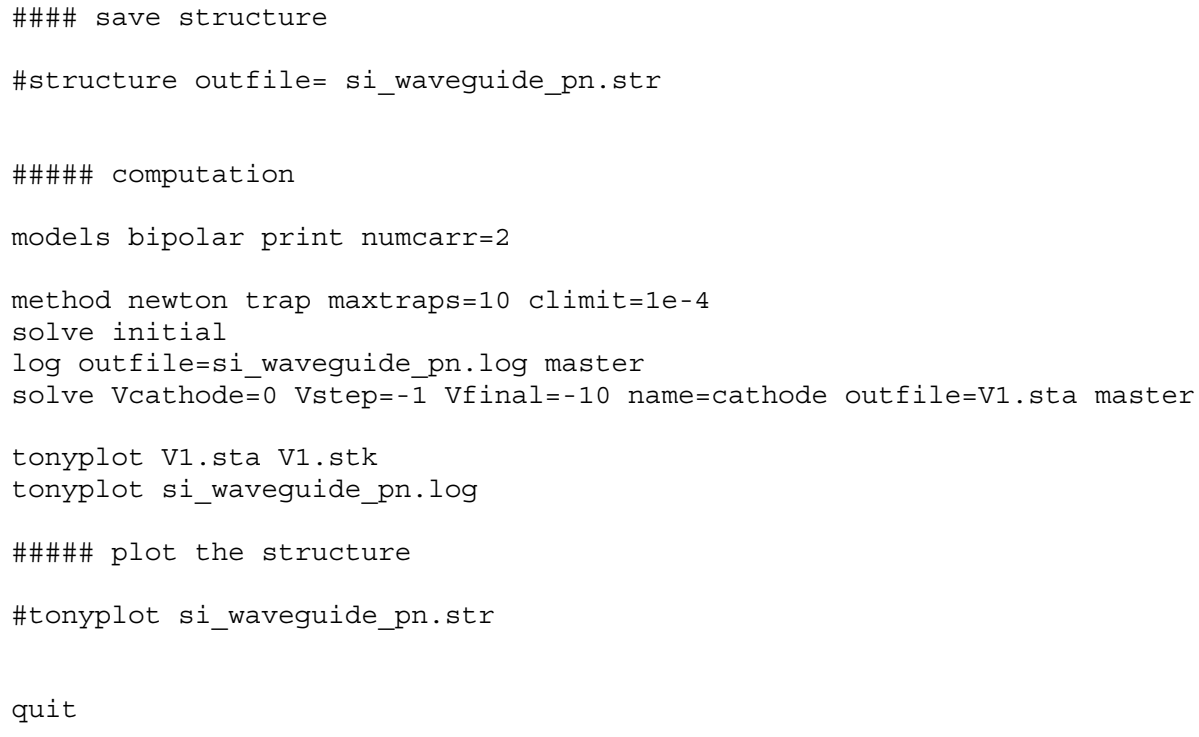

\section{A.2 Conversion code from ATLAS to BEAMPROP/BANDSOLVE}

Hereafter is given an example of the matlab source code, which converts the change in free carrier concentration calculated via ATLAS $^{(\mathrm{TM})}$ into a change in real and imaginary refractive index coefficients matrix compatible with either BEAMPROP $^{(\mathrm{TM})}$ or BANDSOLVE(TM).

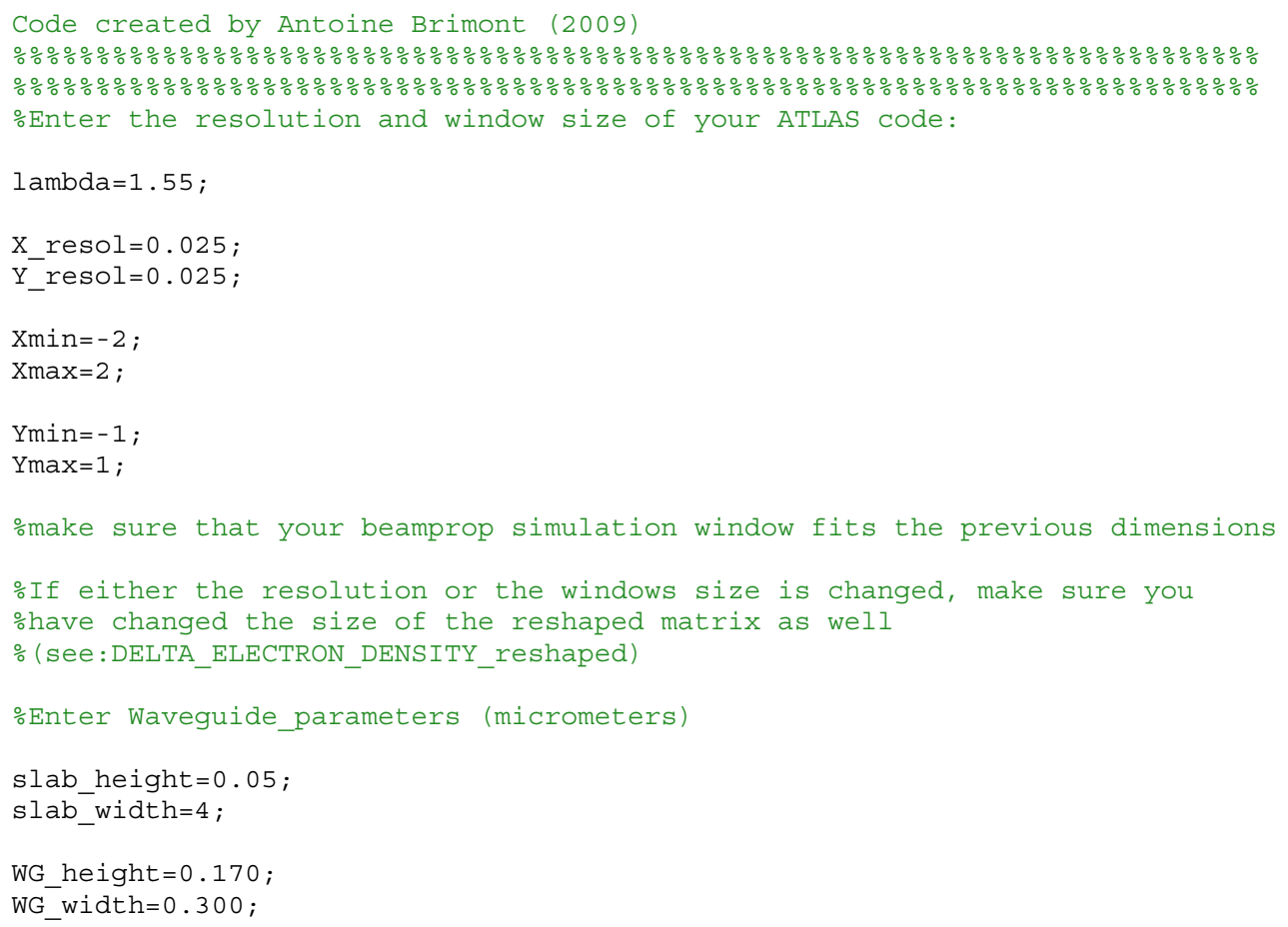




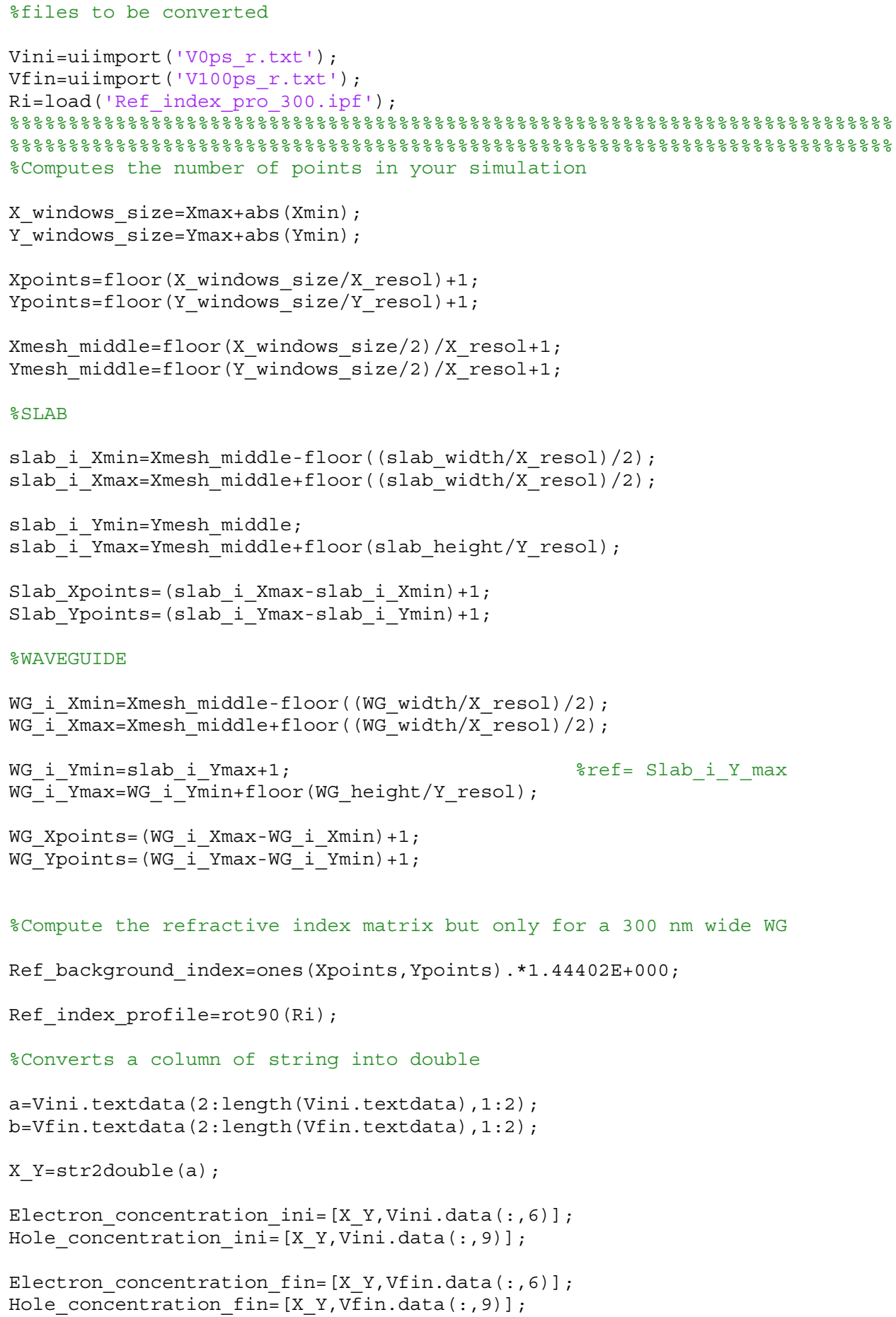


$Y(i)=10000$;

end

end

Del_dup_E $=$ find $($ Electron_conc_fin $==10000)$;

Del_dup_ $X=$ find $(X==10000)$;

Del_dup_Y $=$ find $(X==10000)$;

Electron_conc_fin (Del_dup_E) $=[]$;

$\mathrm{X}($ Del_dup_X $)=[]$;

$\mathrm{Y}\left(\mathrm{Del}{ }_{-}\right.$dup_Y $)=[]$;

OINITIAL CONCENTRATION

Y_Electron_concentration_ini=Electron_concentration_ini $(:, 2)$;

Electron_conc_ini=Electron_concentration_ini $(:, 3)$;

for $i=1$ :length (Electron_concentration_ini)-1;

if Y_Electron_concentration_ini(i)-Y_Electron_concentration_ini $(i+1)==0$

Electron_conc_ini $(i)=10000$;

end

end

Del_dup_E= find $\left(E l e c t r o n \_c o n c \_i n i==10000\right)$;

Electron_conc_ini (Del_dup_E) $=[]$;

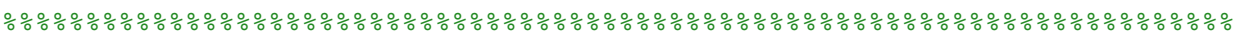

。FINAL ELECTRON DENSITY-INITIAL ELECTRON DENSITY

ELECTRON_DENSITY_INI=reshape (Electron_conc_ini, Ypoints, Xpoints) ;

ELECTRON_DENSITY_FIN=reshape (Electron_conc_fin, Ypoints, Xpoints) ;

DELTA_ELECTRON_DENSITY=Electron_conc_fin-Electron_conc_ini ;

DELTA_ELECTRON_DENSITY_reshaped=reshape (DELTA_ELECTRON_DENSITY,Ypoints,Xpoints) ;

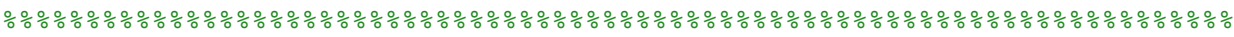

\%1. HOLE DENSITY VARIATION

OFINAL CONCENTRATION

Y Hole_concentration fin=Hole concentration fin $(:, 2)$;

Hole_conc_fin=Hole_concentration_fin $(:, 3)$;

for $i=1$ :length (Hole_concentration_fin) -1;

if Y_Hole_concentration_fin(i) $Y_{-}{ }_{\text {Hole_concentration_fin }(i+1)==0}$

Hole_conc_fin $(i)=10000$;

$\mathrm{X}(\mathrm{i})=1000 \overline{0}$;

$\mathrm{Y}(\mathrm{i})=10000$;

end

end

Del_dup_E= find (Hole_conc_fin==10000);

Del dup $\mathrm{X}=$ find $(\mathrm{X}==1 \overline{0} 000)$;

Del_dup_Y $=$ find $(\mathrm{X}==10000)$;

Hole conc fin (Del dup $E)=[]$;

$\mathrm{X}(\mathrm{De} \overline{\mathrm{I}}$ _dup_X $\mathrm{X})=[]$;

$\mathrm{Y}\left(\mathrm{Del}{ }_{-}\right.$dup_Y $)=[]$;

○INITIAL CONCENTRATION

Y_Hole_concentration_ini=Hole_concentration_ini $(:, 2)$;

Hole_conc_ini=Hole_concentration_ini $(:, 3)$; 
for $i=1$ :length (Hole_concentration_ini) -1;

if Y_Hole_concentration_ini (i) -Y_Hole_concentration_ini $(i+1)==0$

Hole_conc_ini $(i)=10000$;

end

end

Del_dup_E= find (Hole_conc_ini $==10000)$;

Hole_conc_ini (Del_dup_E) $=[]$;

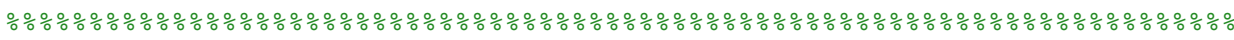
\%FINAL HOLE DENSITY-INITIAL HOLE DENSITY

HOLE_DENSITY_INI=reshape (Hole_conc_ini,Ypoints,Xpoints) ;

HOLE_DENSITY_FIN=reshape (Hole_conc_fin, Ypoints, Xpoints) ;

DELTA_HOLE_DENSITY=Hole_conc_fin-Hole_conc_ini ;

DELTA_HOLE_DENSITY_reshaped=reshape (DELTA_HOLE_DENSITY,Ypoints, Xpoints) ;

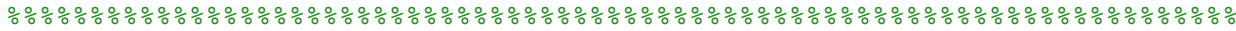

oinitial refractive index profile

Hole conc ini power=Hole conc ini.^^0.8;

HOLE_DENSITY_INI_POWER_RESHAPED=reshape (Hole_conc_ini_power, Ypoints, Xpoints) ;

n_ini $=-(8.8 * 1 \mathrm{E}-22) . *$ ELECTRON_DENSITY_INI $-(8.5 * 1 \mathrm{E}-18) . *($ HOLE_DENSITY_INI_POWER_RESHAPED $)$;

\%final refractive index profile

Hole conc fin power=Hole conc fin.^^0.8;

HOLE_DENSITY_FIN_POWER_RE SHAPĒD=reshape (Hole_conc_fin_power,Ypoints, Xpoints) ;

n_fin $=-(8.8 * 1 \mathrm{E}-22) .{ }^{*}$ ELECTRON_DENSITY_FIN- $(8.5 * 1 \mathrm{E}-18) . *($ HOLE_DENSITY_FIN_POWER_RESHAPED $)$;

Delta $\mathrm{n}=-(8.8 * 1 \mathrm{E}-22) . *($ ELECTRON DENSITY FIN-ELECTRON DENSITY INI $)-(8.5 * 1 \mathrm{E}-$

$18) . *($ HOLE_DENSITY_FIN_POWER_RESHAPED-HOLE_DENSITY_INI_POWER_RESHAPED) ;

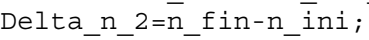

$\% \%$ ATTENTION AUX PARENTHESSES! ! ! ! ! \% \%

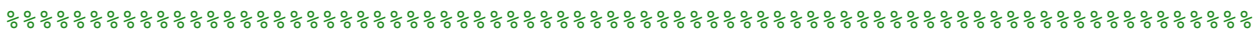

oinitial absorption profile

absorption_ini $=8.5 * 1 \mathrm{E}-18 .{ }^{*} \mathrm{ELECTRON}$ _DENSITY_INI $+6.0 * 1 \mathrm{E}-18 . * \mathrm{HOLE}$ _DENSITY_INI; \% $\mathrm{Cm}-1$

oinitial imaginary refractive index

$\mathrm{K}$ ini=absorption ini.*lambda*1/(4*pi);

K_ini $=K_{-}$ini. *1E-4

\%final absorption profile

absorption_fin $=8.5 * 1 \mathrm{E}-18 . *$ ELECTRON_DENSITY_FIN $+6.0 * 1 \mathrm{E}-18 . *$ HOLE_DENSITY_FIN; $\% \mathrm{CM}-1$

\%final imaginary refractive index

K fin=absorption fin. *lambda*1/(4*pi);

K_fin $=K_{-}$fin. * $1 \mathrm{E}-4$

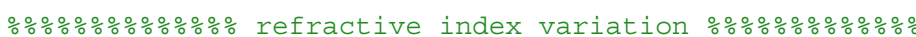

Delta_n=n_fin-n_ini;

Delta_n_rot $=$ rot 90 (Delta_n, 3) ;

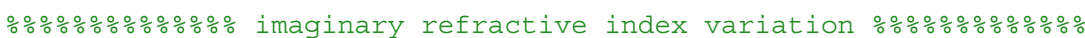

Delta_alpha=absorption_fin-absorption_ini; $\% \mathrm{~cm}-1$

Delta $\mathrm{K}=\mathrm{K}$ fin-K ini

Delta_K_rot $=\operatorname{rot} \overline{9} 0($ Delta_K, 3) ; 


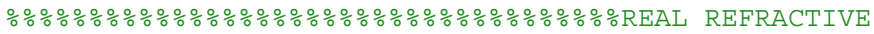

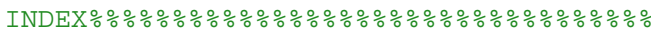

\%WG+SLAB INI (FULL MATRIX INCLUDING BACKGROUND INDEX)

Initial_refractive index=Ref index profile+n ini;

Initial_refractive_index_rot=rot90(Initial_réfractive_index, 3) ;

\%WG INI

Initial_refractive_index_WG_rot=rot90(Initial_refractive_index,3);

Initial_refractive_index_WG_rot (:,slab_i_Ymin:slab_i_Ymax)=Initial_refractive_index_WG_r ot $(1,1) \bar{i} \%$ eliminatès the $\bar{s} \mathbf{l} \overline{\mathrm{a}} \mathrm{b}$

Ini_WG_BPM=Initial_refractive_index_WG_rot-Ref_background_index;

$\div$ SLAB INI

Initial_refractive_index_SLAB_rot=rot90 (Initial_refractive_index,3);

Initial_refractive_index_SLAB_rot (:,WG_i_Ymin:WG_i_Ymax) $=$ Iñitial_refractive_index_WG_rot

$(1,1)$; $\frac{\circ}{\circ}$ liminates the $\mathrm{WG}$

Ini_SLAB_BPM=Initial_refractive_index_SLAB_rot-Ref_background_index;

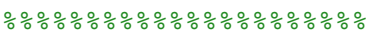

\%WG+SLAB FIN (FULL MATRIX INCLUDING BACKGROUND INDEX)

Final refractive index $=$ Ref index profile+n fin;

Final_refractive_index_rot=rot90(Final_refractive_index, 3 );

\%WG FIN

Final_refractive_index_WG_rot=rot90 (Final_refractive_index, 3);

Final_refractive_index_WG_rot (:, slab_i_Ymin:slab_i_Ymax)=Final_refractive_index_WG_rot (1

,1); \%eliminates the slab

Fin_WG_BPM=Final_refractive_index_WG_rot-Ref_background_index;

○LAB FIN

Final refractive index SLAB rot=rot90 (Final refractive index, 3);

Final_refractive_index_SLAB_rot (:,WG_i_Ymin:WG_i_Ymax) $=$ Final_refractive_index_WG_rot (1, 1

); $\%$ liminates the WG

Fin_SLAB_BPM=Final_refractive_index_SLAB_rot-Ref_background_index;

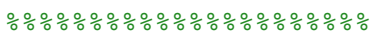

Difference refractive index=Ref index profile+Delta n;

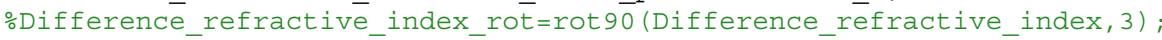

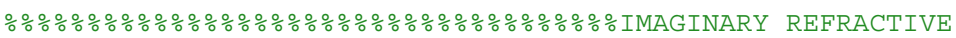

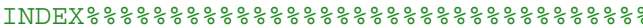

\%WG+SLAB INI (FULL MATRIX INCLUDING BACKGROUND INDEX)

Initial imaginary index $=\mathrm{K}$ ini ;

Initial_imaginary_index_rot=rot90 (Initial_imaginary_index, 3) ;

$\%$ WG INI

Initial imaginary index WG rot=rot90 (Initial imaginary index, 3) ;

Initial_imaginary_index_WG_rot (:,slab_i_Ymin:slab_i_Ymax)=Initial_imaginary_index_wG_rot $(1,1) ; \bar{o}$ liminates the $\overline{\mathrm{s}} \mathrm{l} \mathrm{a} \overline{\mathrm{b}}$

Ini_WG_BPM_imaginary=Initial_imaginary_index_WG_rot ;

$\because$ SLAB INI

Initial_imaginary_index_SLAB_rot=rot90(Initial_imaginary_index,3) ;

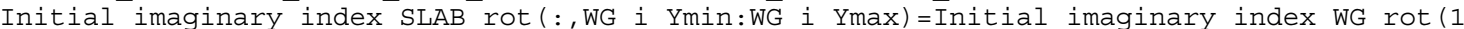

,1) ; \%eliminates the WG

Ini_SLAB_BPM_imaginary=Initial_imaginary_index_SLAB_rot ;

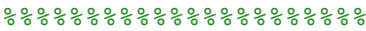

\%WG+SLAB FIN (FULL MATRIX INCLUDING BACKGROUND INDEX)

Final imaginary index $=\mathrm{K}$ fin;

Final_imaginary_index_rot=rot90 (Final_imaginary_index, 3 ); 


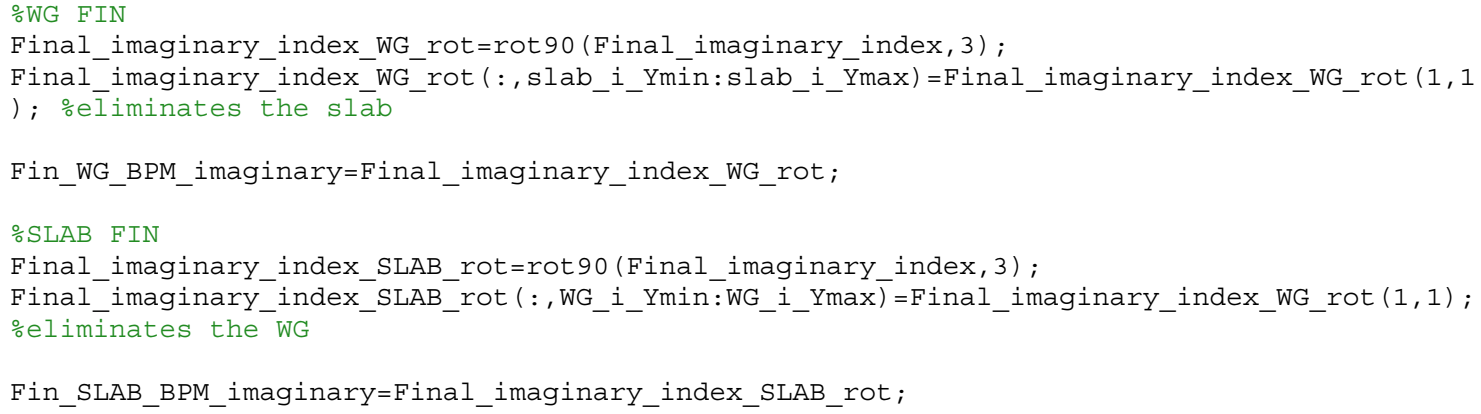


xlabel('X', 'Fontsize', 30,'Fontweight', 'Bold'); ylabel ('Y', 'Fontsize', 30, 'Fontweight', 'Bold'), $\mathrm{X} \lim ([-1,1])$

Ylim $([-0.5,0.5])$

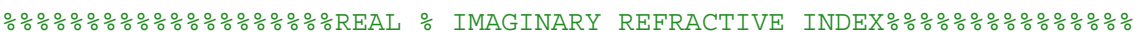

\%WG INI

fid = fopen('WG_THIN_0ps_6V.lpf', 'wt'); \% Open for writing

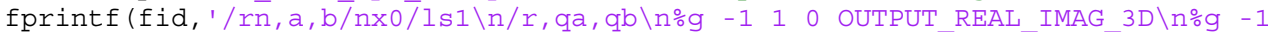
1 ',WG_Xpoints,WG_Ypoints);

for i=WG_i_Xmin:WG_i_Xmax

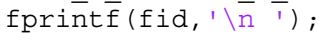

for $u=W G$ i Ymin:WG i Ymax

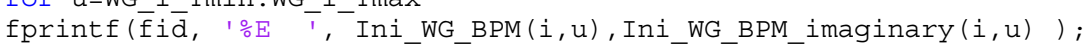

fclose (fid) ;

$\because$ SLAB INI

fid = fopen('SLAB_THIN_0ps_6V.Ipf', 'wt'); \% Open for writing fprintf (fid, '/rn, $\mathrm{a}, \mathrm{b} / \mathrm{nx} 0 / \mathrm{ls} \mathrm{l} \backslash \mathrm{n} / \mathrm{r}, \mathrm{qa}, \mathrm{qb} \backslash \mathrm{n} \div \mathrm{g}-1 \quad 1 \quad 0$ OUTPUT REAL IMAG $3 \mathrm{D} \backslash \mathrm{n} \% \mathrm{~g}-1$ 1',slab_Xpoints,Slab_Ypoints);

for i=slab_i_Xmin:slab_i_Xmax fprintéf(fid, '\n ') $\overline{\text { i }}$

for $u=s l a b$ i Ymin:slab i Ymax

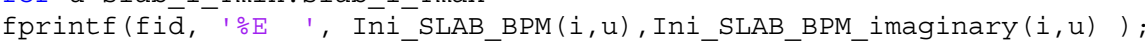

end

end

fclose (fid)

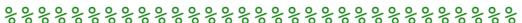

\%WG FIN

fid = fopen ('WG THIN 100ps 6V.lpf', 'wt'); \% Open for writing

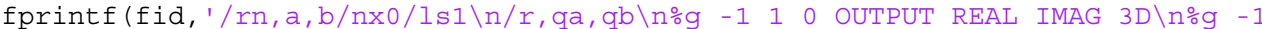
1 ',WG_Xpoints, WG_Ypoints);

for $i=W G$ i_Xmin:WG_i_Xmax fprintf (fid, ' $\backslash \bar{n}-$ ') ;

for $u=W G$ i Ymin:WG i Ymax

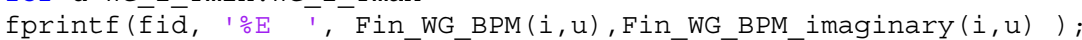

end

end

fclose (fid);

$\because$ SLAB FIN

fid = fopen('SLAB THIN 100ps 6V.lpf', 'wt'); \% Open for writing

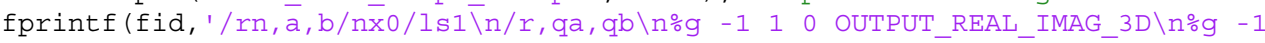

1',Slab Xpoints, Slab_Ypoints);

for i=slab_i_Xmin:slab_i_Xmax

fprintf(fid, ' \n ');

for $u=s l a b$ i_Ymin:slab_i_Ymax

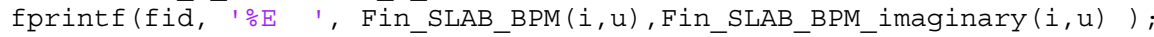

end

fclose (fid); 


\section{Appendix B}

\section{B.1 Modulator masks}

The slow wave modulator masks shown in Fig B-1 consist of 9 different layers, which are used for $\mathrm{Si}$ waveguide and fiber coupler definition, $\mathrm{SiO}_{2}$ window opening and electrode patterning as well as for implantation of two different species (Boron and Phosphorus) at different concentrations (P, N, $\mathrm{P}^{+}$and $\mathrm{N}^{+}$). 26 modulators of varying length and reference waveguides fit onto a $7.40 \times 8.40 \mathrm{~mm}^{2}$ silicon chip. The exposure dose $\left(\right.$ in $\mathrm{mJ} / \mathrm{cm}^{2}$ ) is varied along the different the 13 columns of a $200 \mathrm{~mm}$ Si wafer to provide a variety of slow wave waveguide dimensions. The more intense the illumination dose, the smaller the dimensions.

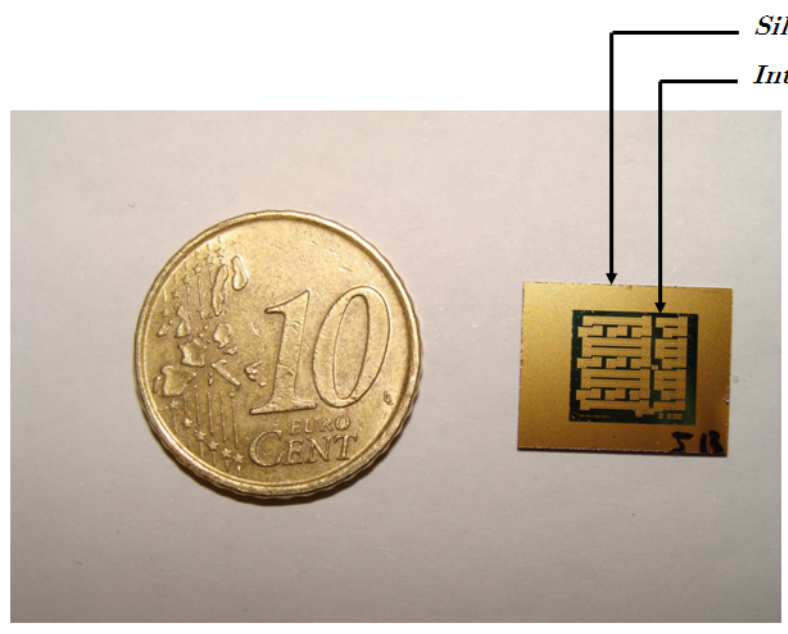

(a)

Silicon chip Overlapped Mask layers Integrated silicon modulators

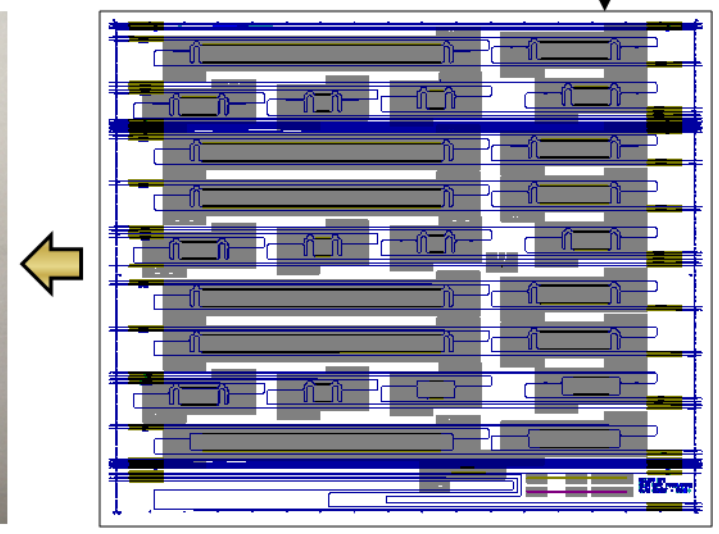

(b)

Figure B-1: (a) Picture of a single silicon chip consisting of 26 electro-optical modulators and (b) corresponding mask layers. 


\section{Appendix C}

\section{C.1 Publications in peer-reviewed journals}

[J.1] A. Brimont, D. J. Thomson, P. Sanchis, J. Herrera, F. Y. Gardes, J. M. Fedeli, G.T. Reed, and J. Marti, "High speed silicon electro-optical modulators enhanced via slow light propagation," Opt. Express 19, 2087620885 (2011)

[J.2] G. Rasigade, M. Ziebell, D. Marris-Morini, A. Brimont, A. M. G. Campo, P. Sanchis, J. M. Fedeli, G. H. Duan, E. Cassan, and L. Vivien, "10-Gb/s Error-Free Silicon Optical Modulator for Both TE and TM Polarized Light," Photonics Technology Letters, IEEE 23, 1799-1801 (2011).

[J.3] A.M. Gutierrez, A. Brimont, G. Rasigade, M. Ziebell, D. Marris-Morini, J-M. Fédéli, L. Vivien, J. Marti and P. Sanchis, "Ring-assisted MachZehnder interferometer silicon modulator for enhanced performance", accepted in J. Lightwave Technol. (2011).

[J.4] A. Brimont, J. Vicente Galán, J. Maria Escalante, J. Martí, and P. Sanchis, "Group-index engineering in silicon corrugated waveguides," Opt. Lett. 35, 2708-2710 (2010).

[J.5] A. Brimont, P. Sanchis, and J. Martí, "Strong electro-optical modulation enhancement in a slow wave corrugated waveguide," Opt. Express 17, 9204-9211 (2009). 
[J.6] F. Y. Gardes, A. Brimont, P. Sanchis, G. Rasigade, D. Marris-Morini, L. O'Faolain, F. Dong, J. M. Fedeli, P. Dumon, L. Vivien, T. F. Krauss, G. T. Reed, and J. Martí, "High-speed modulation of a compact silicon ring resonator based on a reverse-biased pn diode," Opt. Express 17, 2198621991 (2009).

[J.7] A. Brimont, P. Sanchis, and J. Marti, "High speed silicon modulators: one step further toward VLSI photonics," ITECKNE 4, 51-54 (2007).

\section{C.2 Other contributions in peer-reviewed journals}

[J.8] P. Sanchis, A. Brimont, A. Gutierrez, M. Aamer, "A method for experimentally estimating the insertion losses of slow-light photonic waveguiding structures", submitted (2011).

[J.9] A.M. Gutierrez, A. Brimont, M. Aamer, P. Sanchis, "Method for measuring waveguide propagation losses by means of a Mach-Zehnder Interferometer structure", accepted in Opt. Communications (2011)

[J.10] P. Sanchis, P. Villalba, F. Cuesta, A. Håkansson, A. Griol, J. V. Galán, A. Brimont, and J. Martí, "Highly efficient crossing structure for siliconon-insulator waveguides," Opt. Lett. 34, 2760-2762 (2009).

\section{C.3 National and international conferences}

[C.1] A. Brimont, D. J. Thomson, P. Sanchis, J. Herrera, F. Y. Gardes, J. M. Fedeli, G. T. Reed, and J. Marti, "Enhancing the performance of carrier depletion based silicon electro-optical modulators via slow light propagation," Post deadline paper in Group IV Photonics (GFP), 8th IEEE International Conference on, 1-3. (2011)

[C.2] J. V. Galan, T. Tekin, G. B. Preve, A. Brimont, M. Llopis, and P. Sanchis, "Low profile silicon photonics packaging approach featuring configurable multiple electrical and optical connectivity," in Group IV Photonics (GFP), 8th IEEE International Conference on, 377-379 (2011) 
[C.3] M. Aamer, A.M. Gutiérrez, A. Brimont, P. Sanchis "Ring Resonator Structures for Active Applications in Silicon", VII reunion española de optoelectronica (2011).

[C.4] D. J. Thomson, F. Y. Gardes, G. T. Reed, L. Vivien, D. Marris-Morini, G. Rasigade, A. Brimont, P. Sanchis, J. M. Fédéli, L. O'Faolain, T. F. Krauss, L. Lever, Z. Ikonic, and R. Kelsall, "Silicon optical modulators for high data rate applications," Invited paper in Group IV Photonics (GFP), 8th IEEE International Conference on (2011), 5-7, (2011)

[C.5] D. J. Thomson, F. Y. Gardes, Y. Hu, A. Ahmed, G. T. Reed, G. Rasigade, M. Ziebell, D. Marris-Morini, L. Vivien, A. Brimont, P. Sanchis, J. M. Fedeli, F. Milesi, and C. Min, "Silicon-based optical modulation within the HELIOS project," in Silicon Photonics VI, (SPIE), 794316-794311 (2011).

[C.6] J. Garcia-Ruperez, J. Garcia-Castello, V. Toccafondo, and A. Brimont, "Sensing Technique for the Development of Real-time and Low-cost Biosensors Using Photonic Bandgap Structures," in Optical Sensors, OSA Technical Digest (CD) (Optical Society of America, 2011), SWB5.

[C.7] A. Brimont, J. Marti, and P. Sanchis, "Slow wave and resonator structures to enhance silicon photonic modulators performance," Proceedings 2nd Conferencia Española de Nanofotónica (2010).

[C.8] A. Brimont, A. Griol, J. Marti, and P. Sanchis, "Group index engineering in silicon corrugated waveguides," in Group IV Photonics (GFP), 7th IEEE International Conference on, 39-41 (2010).

[C.9] F. Y. Gardes, D. Thomson, G. T. Reed, L. O'Faolain, T. F. Krauss, L. Lever, R. Kelsall, Z. Ikonic, D. Marris-Morini, G. Rasigade, L. Vivien, A. Brimont, P. Sanchis, F. Milesi, and J. M. Fedeli, "Undertaking research in the field of silicon optical modulators in the framework of the Helios and UK silicon photonics projects," Invited paper in Group IV Photonics (GFP), 7th IEEE International Conference on, 192-194 (2010). 
[C.10] A. Brimont, P. Sanchis, J. V. Galan, J. M. Fedeli, A. M. Gutierrez, and J. Marti, "Experimental demonstration of moderately low group velocity in silicon rib photonic Wire Bragg Gratings," Proceedings 23rd Annual Meeting of the IEEE Photonics Society, 713-714 (2009).

[C.11] F. Y. Gardes, A. Brimont, P. Sanchis, G. Rasigade, D. Marris-Morini, L. O'Faolain, F. Dong, J. M. Fedeli, P. Dumon, L. Vivien, T. F. Krauss, G. T. Reed, and J. Martí, "High-speed modulation of a compact silicon ring resonator," in Group IV Photonics (GFP), 7th IEEE International Conference on, 241-243 (2009).

[C.12] J. V. Gálan, P. Sanchis, J. Garcia, A. Martinez, J. Blasco, J. M. Martinez, A. Brimont, and J. Marti, "Silicon cross-slot waveguides insensitive to polarization.," Proceedings IEEE/LEOS Winter Topicals Meeting Series (2009).

[C.13] A. Brimont, P. Sanchis, F. Y. Gardes, G. T. Reed, D. Marris-Morini, L. Vivien, P. Dumon, W. Bogaert, L. O'Faolain, T. F. Krauss, and J. M. Fedeli, "SOI micron-size ring resonator optical modulator," Epixnet spring shool (2008).

[C.14] A. Brimont, F. Y. Gardes, P. Sanchis, D. Marris-Morini, P. Dumon, J. M. Fedeli, L. O'Faolain, W. Bogaert, L. Vivien, J. Martí, G. T. Reed, and T. F. Krauss, "Design of a micro-ring resonator electro-optical modulator embedded in a reverse biased PN junction," Proceedings 14th European Conference on Integrated Optics 321-324 (2008).

[C.15] P. Sanchis and A. Brimont, "Route to develop high performance compact silicon modulators," European Photonic Integration Forum (2008).

[C.16] P. Sanchis, J. V. Galan, A. Brimont, A. Griol, and J. Marti, "Low crosstalk in silicon-on-insulator waveguide crossings with optimized angle," Proceedings 4th Conference on Group IV photonics, 159-161 (2007). 


\section{List of Figures}

Figure 1-1: Examples of two random combinations of primitive vectors in a cubic lattice structure ................................................................................... 2

Figure 1-2: Diamond lattice structure.......................................................... 2

Figure 1-3: Brillouin zone of the diamond lattice structure ............................. 3

Figure 1-4: Simplified band structure of (a) semiconductors (b) Insulators and (c) Metals. $E_{c}$ denotes the bottom of the conduction band, $E_{v}$ the top of the valence band and $E_{F}$ the Fermi level. The Fermi level is defined as the highest occupied

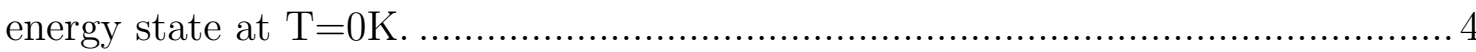

Figure 1-5: Band structure of an intrinsic semiconductor in the k-space ............... 5

Figure 1-6: Calculated silicon band structure. The valence band is split in two bands with wider/narrower curvature corresponding to heavy/light holes, respectively (blues crosses). The conduction electrons lie in the conduction band (red minuses). (After [10])

Figure 1-7: Schematic representation of (a) intrinsic silicon, (b) p-type silicon with acceptor impurity (boron) and (c) n-type silicon with acceptor impurity (Phosphorus)

Figure 1-8: Schematic of a band structure with donor and acceptor energy levels 7

Figure 1-9: schematic representation of an ideal abrupt silicon $p n$ junction .......... 8

Figure 1-10: $p n$ junction at equilibrium ......................................................... 9

Figure 1-11: Abrupt pn junction at thermal equilibrium (a) energy band diagram. (b) space-charge distribution. (c) Potential as a function of the distance. $V_{b i}$ is the built-in potential, (d) Electric field distribution .......................................... 9

Figure 1-12: (a) Energy band diagram under forward bias. (b) Carrier distribution $u n d e r$ forward bias......................................................... 12 
Figure 1-13: (a) Energy band diagram under reverse bias. (b) Carrier distributions under reverse bias

Figure 1-14: Current densities in a PN junction ............................................... 14

Figure 1-15: Current voltage characteristics of an ideal $p n$ junction.................... 15

Figure 1-16: Current voltage characteristics of a real Si diode (a) generationrecombination current region. (b) Diffusion current region. (c) High-injection region. (d) Series resistance effect. (e) Reverse leakage current due to generationrecombination and surface effects. Picture taken from reference [12] .................. 16

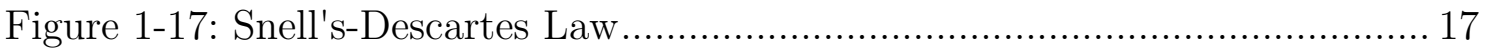

Figure 1-18: Light guidance by total internal reflection in a 2D slab waveguide

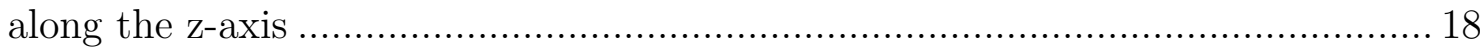

Figure 1-19: TE (TM) polarized rays: the electric (magnetic) field is perpendicular to plane of incidence. 18

Figure 1-20: Extension of the ray optics approach associating a propagating wave following the paths traced by the ray.... 19

Figure 2-1: Basic working principle of a mechano-optical modulator.... 24

Figure 2-2: (a) Analog (b) digital amplitude modulation in a MZI modulator. ... 24

Figure 2-3: Graphical illustration of the extinction ratio and insertion loss..... 26

Figure 2-4: Theoretical eye patterns of optical modulators featuring respectively (a) $40 \mathrm{GHz}$, (b) $30 \mathrm{GHz}$, (c) $20 \mathrm{GHz}$, and (d) $10 \mathrm{GHz} 3 \mathrm{~dB}$ EO bandwidths and driven under a NRZ PRBS ${ }^{-1} 40$ Gbits/s electrical signal [15].

Figure 2-5: Basic principle of operation of an EO MZI modulator (a) The phase shifters (in red) are unbiased and the propagating waves experience no phase difference. Both light waves recombine at the MZI output in the form of a constructive interference. The EO modulator is the "on-state" (b) One of the phase shifter is biased and the wave propagating through the altered waveguiding medium experiences a phase shift ( $\pi$ for maximum extinction). Both light waves recombine at the MZI output in the form of a destructive interference. The EO modulator is the "off-state"

Figure 2-6: Projected Energy per bit consumption of a $50 \Omega$ impedance matched TW MZI modulator versus drive voltage for varying bit rate. 32

Figure 2-7: (a) Fundamental photon absorption process in a bulk semiconductor at thermal equilibrium. Electronic transitions occur at photon energies equal to or higher than the band gap (b) The energy band tilting under a strong electric field 
yields to the Franz-Keldysh absorption process. Electronic transitions occur at photon energies lower than the band gap $E_{g}$.....

Figure 2-8: Schematic description of the Quantum Stark effect (a) Quantum well under no applied electric field (b) Quantum well under an applied electric field; the energy required to make a transition $E_{t r a n}$ decrease and the absorption edge is shifted towards longer wavelengths. 38

Figure 2-9: (a) Refractive index change versus free carrier concentration variations calculated from the Soref's empirical model (respectively Drude's theoretical model) induced by electrons (1) (resp. (2)) and holes (3) (resp. (4). (b) Absorption coefficient versus free carrier concentration variations calculated from the Soref's empirical model (respectively Drude's theoretical model) induced by electrons (1) (resp. (2)) and holes (3) (resp. (4)).

Figure 2-10: (a) $L_{\pi}$ (Length to achieve a $\pi$-phase shift) variations (blue plain line) plotted together with the loss in dB.cm ${ }^{-1}$ (red dashed line) induced by free hole concentration variations based on the Soref empirical model. (b) $L_{\pi}$ variations (blue plain line) plotted together with the net loss (red dashed line) induced by free hole concentration variations based on the the Soref empirical model. In both cases, $\alpha_{0}=0.691 \mathrm{~cm}^{-1}$, which is equivalent to $3 \mathrm{~dB} . \mathrm{cm}^{-1}$. It is worth noting that this loss figure is highly dependent upon the fabrication process and/or waveguide dimensions and hence may be lowered. (via decreasing sidewall roughness, for instance).

Figure 2-11: White dashed contour lines depict an optical mode profile overlapping with a refractive index change distribution achieved via depletion of a $p n$ junction in a sub-micrometer rib waveguide. The mode was calculated with Beamprop $^{\mathrm{TM}}$ and the real (resp. imaginary) refractive index change extracted via Soref's Equations (1.63) and (1.64) from the free carrier densities, itselves simulated with ATLAS ${ }^{\mathrm{TM}}$....

Figure 2-12: Overview of the procedure to simulate electro-optical modulators based on active periodic structure. (a) 3D passive design using PWE algorithm, (a) 2D Electrons and hole densities are converted via a in "in-house" code into refractive index distributions via Soref's Equations (1.63) and (1.64) (c) This densities are included back into the 3D PWE simulator to calculate the (d) modal effective index change for varying refractive index distribution. 
Figure 2-13: "Standard" fiber-to-the-chip coupling techniques. (a) Vertical coupling schemes via grating couplers (b) Horizontal (butt) coupling scheme..... 58

Figure 2-14: Schematic description of a two port network 59

Figure 2-15: Schematic of the characterization setup to determine the S21 electrooptical response.....

Figure 2-16: Pictures of the characterization setup to determine the S21 electrooptical response.

Figure 2-17: Schematics of the characterization setup for eye diagram acquisition and BER measurements.....

Figure 2-18: Pictures of the characterization setup for eye diagram acquisition and BER measurements.

Figure 3-1: (a) one dimensional PhCs. (b) two dimensional $\mathrm{PhC}$ with line defect. (c) three dimensional « wood pile» $\mathrm{PhC}$.

Figure 3-2: (a) Band diagram of a LCWG. The blue solid lines represent the first three eigen odd modes with respect to the plane of symmetry of the structure $\mathrm{X}=0$ as explained further in section 3.3.2. Only those located below the light line (green dashed) propagate through the optical structure theoretically without losses. (b) Slow light propagation by omnidirectional reflections and (c) backscattering. 68

Figure 3-3: Electric field vectors predominantly (a) perpendicular to the mirror plane of the structure $(\mathrm{X}=0)$ and consistent with odd/TE-like modes (b) parallel to the mirror plane $(\mathrm{X}=0)$ and consistent with even/TM-like modes. (c) parallel to another mirror plane of the structure $(Y=0)$ and consistent with even/TE-like modes (d) perpendicular another mirror plane of the structure $(\mathrm{Y}=0)$ and consistent with odd/TM-like modes.

Figure 3-4: Examples of compensation for optical proximity effects in a (a) 2D triangular lattice PhCs and (b) 1D laterally corrugated waveguide. The blue structures stand for the design altered in advance and the red dashed line is the actual printed structure

Figure 3-5: Schematic description of the different extrinsic loss mechanisms occurring (a) LCWGs (b) W1 PhCs. In (a) and (b), the green arrows illustrates the forward wave propagation motion. The yellow arrows depict the coupling losses due to fast to slow wave impedance mismatch. Note that this source of losses may be minimised by convenient tapering (not represented here) at both 
input and output of the waveguides $[110,113]$. The blue arrows depict the backscattered light due to waveguide defects (roughness, positional disorder) interfering destructively with the slow forward propagating wave. The red arrows describe in the scattering losses due to fabrication imperfections (roughness, disorder): in (a) light scatters freely in all directions into the cladding. (b) Because the allowed modes inside the line defect are confined by the photonic band gap in-plane light is has no choice but escaping in the vertical direction through the holes, i.e. where there is a lack of confinement.

Figure 3-6: Footprint of a (a) W1 PhC with triangular lattice with 9 periods repeated on both side of the defect (b) LCWGs

Figure 3-7: The red shaded line depicts the unit cell of a (a) W1 PhC with triangular lattice with 9 period repeated on both side of the defect (b) LCWGs. The unit cell is extended on both side of the periodic structure to avoid coupling with their periodically repeated neighbors. Although not depicted here, the same holds in the vertical direction (y -axis).

Figure 3-8: : Schematic of a (a) deep-etched LCWG defined by a set of 4 parameters: waveguide Height $(H)$, narrow and wide section widths, respectively $W$ and $W e$ and period $\Lambda$ and (b) shallow-etched LCWG with slab height (h) ....78 Figure 3-9: Electric field vectors predominantly (a) perpendicular to the mirror plane of the structure $(\mathrm{X}=0)$ and consistent with odd/TE-like modes (b) parallel to the mirror plane $(\mathrm{X}=0)$ and consistent with even/TM-like modes. 78

Figure 3-10: Top down view of the parameterized shallow-etched LCWG (the slab is not represented here).

Figure 3-11: 2D cut of the fundamental mode profiles propagating through (a) $300 \mathrm{~nm}$ and (b) $550 \mathrm{~nm}$ wide waveguides @ $1.55 \mu \mathrm{m}$ wavelength. The effective index values are respectively (a) $n_{\text {eff, }},=2.109451$ and $n_{\text {eff, }}$ we $=2.55557$. . .

Figure 3-12: Contour plot of the Bragg wavelength @ the photonic band edge versus width $\left(W_{e}\right)$ and length $\left(W_{i}\right)$ of the transversal corrugations, respectively for the (a) fundamental and (b) first order mode. Corresponding top views of the square electric field of the (c) fundamental and (d) first order Bloch modes. $\Lambda=$ $330 \mathrm{~nm}, W=300 \mathrm{~nm}, h=50 \mathrm{~nm}$.

Figure 3-13: Contour plot of the Bragg wavelength @ the photonic band edge versus width $\left(W_{e}\right)$ and length $\left(W_{i}\right)$ of the transversal corrugations, respectively for 
the (a) first order mode. $(\Lambda=305 \mathrm{~nm}, W=400 \mathrm{~nm})$ (b) fundamental mode $(\Lambda=$ $300 \mathrm{~nm}, W=300 \mathrm{~nm})$ and (c) fundamental mode $(\Lambda=305 \mathrm{~nm}, W=200 \mathrm{~nm}) \ldots \ldots 86$ Figure 3-14: Schematics of the slow wave phase shifter (SWPS) and the fundamental slow mode $\left|E_{x}\right|^{2}$ profile respectively in the narrow and wide section. 88

Figure 3-15: Contour plot of the fundamental mode wavelengths in the slow light regime versus varying periodic elements size for undoped silicon corrugated waveguides. The blue contour line has been derived including the slight refractive index variation at $\lambda=1.55 \mu \mathrm{m}$ due to the impurities introduced into the waveguide. Results are plotted at the band edge $\left(k=0.5^{*} 2 \pi / \mathrm{a}\right)$. 89

Figure 3-16: Design method flow for the DC analysis of the slow wave modulator

Figure 3-17: real refractive index change $\Delta n$ under different applied drive voltages (a), (a') $\Delta V=-2 \mathrm{~V}$; (b), (b') $\Delta V=-4 \mathrm{~V} ;$ (c), (c') $\Delta V=-6 \mathrm{~V} ;$ (d), (d') $\Delta V=-8 \mathrm{~V}$; (c), (c') $\Delta V=-10 \mathrm{~V}$ for the thin and wide sections of the LCWG, respectively. The junction is in steady state. Waveguide cross section boundaries are represented by the white plain lines

Figure 3-18: DC response of the slow wave phase shifter: Effective index change versus applied reverse bias for varying group index

Figure 3-19: Figure of merit versus applied reverse bias calculated from Equation (4.7) for varying group index.

Figure 3-20: Interaction length versus applied reverse bias for varying group index

Figure 3-21: Schematic of the reverse biased slow wave single drive amplitude modulator operated in DC. The inset depicts a portion of the phase slow wave shifter. The silicon slab is only represented in the active part of the MZI for clarity.

Figure 3-22: Transmission spectra of a Mach-Zehnder modulator (MZM) with a $500 \mu \mathrm{m}$-long SWPS inserted the active arm for varying applied reverse bias from $V=0 \mathrm{~V}$ (unbiased) to $V=-8 \mathrm{~V}$. (a) depicts the overall transmission spectrum of the MZM. Insets (b), (c), and (d) show portions of spectrum (a) over narrower wavelength ranges to appreciate the phase shift enhancement for increasing group index. 
Figure 3-23: Transmission spectrum of a Mach-Zehnder Modulator (MZM) with a $1500 \mu \mathrm{m}$-long SWPS inserted the active arm for varying applied reverse bias from $V=0 \mathrm{~V}$ (unbiased) to $V=-1 \mathrm{~V}$. 98

Figure 3-24: Schematic of the ramp excitation applied to the modulator 99

Figure 3-25: Design method flow for the transient analysis of the slow wave modulator

Figure 3-26: Transient response of the slow wave phase shifter: Effective index change versus time for varying group index under an applied voltage $V_{\max }=-6 \mathrm{~V}$.

Figure 3-27: Maximum effective index change (red curve) and resulting interaction length (blue curve) against group index for $V_{\max }=-6 \mathrm{~V}$.

Figure 3-28: Figure of merit of the slow wave phase shifter versus group index for a $V_{\max }=-6 \mathrm{~V}$ reverve bias voltage.

Figure 3-29: Free carrier absorption loss versus applied reverse bias voltage for varying group index

Figure 3-30: Scattering loss due to sidewall roughness versus applied reverse bias voltage for varying group index. 106

Figure 3-31: Group index versus wavelength for varying applied reverse bias voltage. (a), (b), (c), depict some examples of the group index variation $\Delta n_{g}=n_{g}$ (bias) $-n_{g}$ (unbiased) where $n_{g}$ (unbiased) $=30,20$, and 10, respectively.... 106

Figure 3-32: Total insertion loss versus applied reverse bias voltage for varying group index

Figure 3-33: (a) Achieved phase shift versus SWPS length for a -6V reverse bias voltage. Inset (b) shows an enlarged view of the region surrounded by a dashed rectangle.

Figure 3-34: Phase shifter loss versus phase shifter length for varying group index assuming a $-6 \mathrm{~V}$ reverse bias voltage

Figure 3-35: (a) Achieved phase shift in a 1mm long phase shifter versus applied drive voltage. Inset (b) shows zoomed in view of the region surrounded by a dashed rectangle.

Figure 3-36: Optical bandwidth (after references [83, 94, 96, 116]) versus group index for a LCWG. Q factor equivalents of ring resonators are indicated for the sake of comparison. 
Figure 3-37: Optimum SWPS length versus applied voltage for varying ER "ontarget". A group index $n_{g}=10$ is assumed.

Figure 3-38: (a)Logarithmic (dBm), (b) group index versus wavelength and (c) linear (a.u.) response of a symmetric MZI with identical SWPS integrated in both arms to balance losses. A group index $n_{g}=10$ is assumed.

Figure 3-39: Tolerance on the group index variation (in \%) versus the worst extinction ER.

Figure 3-40: Optical bandwidth versus Extinction ratio "on-target"

Figure 3-41: Influence of the temperature variation on the group index wavelength dependence

Figure 3-42: Influence of the temperature variation on the SW EOM extinction ratio for varying group index. The corresponding wavelength detuning is also shown on the abscissa.

Figure 3-43: Slow wave modulator electrical design evolution. From asymmetric to self aligned $p n$ junction.

Figure 3-44: Slow wave EOM fabrication process (lithography and stripping intermediate steps are not represented for simplicity) developed by Thomson et al. [49]

Figure 3-45: (a) Transmission spectra of the $0.2 \mathrm{~mm}, 0.5 \mathrm{~mm}$ and $1 \mathrm{~mm}$ long LCWGs. Normalized spectra are shifted vertically by $10 \mathrm{~dB}$ for clarity (b) Waveguide overview (c), (d) SEM images of the actual fabricated LCWGs. The dashed line is the waveguide contour as it appears on the mask.

Figure 3-46: (a) transmission spectra of two MZI loaded with one LCWGs of respective length $L=0.5$ and $0.25 \mathrm{~mm}$ shown in the inset (b) and (c) Calculated group index versus wavelength for respective LCWG lengths of $L=0.5$ and 0.25 $\mathrm{mm}$.

Figure 3-47: (a), (b) and (c) Corrugated waveguides as designed on the mask corresponding respectively to design 5,6 and 7 (d), (e), (f) Top views of the expected (dashed line) and actual corrugated waveguides (SEM images).

Figure 3-48: (a) Taper as designed on the mask (b) Actual taper

Figure 3-49: (a) resp. (b) Transmission spectra of the $0.2 \mathrm{~mm}, 0.5 \mathrm{~mm}, 1 \mathrm{~mm}$ and 2mm -long LCWGs corresponding to design 6 (shallow corrugations) and 7 (deep corrugations). Normalized spectra are shifted vertically by $10 \mathrm{~dB}$ for clarity. Insets are SEM images of the fabricated LCWGs. 
Figure 3-50: Overview of the slow wave MZI. The silicon slab is not depicted for clarity

Figure 3-51: (a) transmission spectra of an assymetric MZI with one doped LCWGs of length $L=1 \mathrm{~mm}$ placed in the shorter arm. Normalized spectra are shifted vertically by $20 \mathrm{~dB}$ for clarity. (b) Calculated group index versus wavelength for a LCWG length of $L=1 \mathrm{~mm}$. The LCWG corresponds here to design 6 (see (a) and (b) of Figure 3-47).

Figure 3-52: (a) transmission spectra of an assymetric MZI with one doped LCWGs of length $L=1 \mathrm{~mm}$ placed in the shorter arm. Normalized spectra are shifted vertically by $20 \mathrm{~dB}$ for clarity. (b) Calculated Group index versus wavelength for LCWG length of $L=1 \mathrm{~mm}$. The LCWG corresponds here to design 7 (see (c) and (f) of Figure 3-47)....

Figure 3-53: (a) Schematic of the modulator based on an asymmetric MachZehnder interferometer (MZI). Multi-mode interference (MMI) structures are used to split and combine the light respectively at the input and output of the MZI.

(b) Transverse scanning electron microscope (SEM) picture of the slow wave modulator with an overview of the ground-signal-ground coplanar (GSG) metal electrodes. (c), (b) Top and transverse SEM pictures of the corrugated waveguide. Left inset shows a zoomed view of the taper used to reduce the coupling losses at the rib to slow wave waveguide transitions. Right inset depicts a zoomed view of the corrugated waveguide. Doping regions are delimited by the colored areas.

Figure 3-54: (a) Normalized transmission spectrum of the slow wave modulator for three different reverse biased voltages. The slow and fast light regions are respectively delimited in green and red. (b) Expanded view of the slow light region. (c) Group index variation versus wavelength at $0 \mathrm{~V}$ and $10 \mathrm{~V}$ bias voltages. (d) Phase shift dependence versus applied bias for varying group index. 140 Figure 3-55: (a) Insertion losses versus group index of the $1 \mathrm{~mm}$ long slow wave phase shifter (b) Insertion loss penalty relative to the conventional modulator. $C_{p}$ accounts for the coupling losses between the slow wave and conventional rib waveguides while $L_{\text {corr }}=1 \mathrm{~mm}$ is the modulation length. Average modulation efficiencies $V_{\pi} L_{\pi}$ and enhancement factor (between 0 and $10 \mathrm{~V}$ ) versus group index of the slow wave modulator are shown. $V_{\pi}$ and $L_{\pi}$ are respectively the bias voltage and modulation length required to achieve a $\pi$ radian phase shift. 
Figure 3-56: Slow wave modulator electro-optical response. (a) Normalized electro-optical frequency response of two MZI modulators with respective slow wave phase shifter lengths of $1 \mathrm{~mm}$ and $0.5 \mathrm{~mm}$. Spectra are shifted vertically by 6 $\mathrm{dB}$ for clarity. (c) Normalized electro-optical frequency response of the $1 \mathrm{~mm}$ long slow wave modulator for varying group index. Spectra are shifted vertically by 4 $\mathrm{dB}$ for clarity. The $3 \mathrm{~dB}$ roll-off bandwidth of the TW slow wave modulator is mainly limited as a result of the velocity mismatch between the electrical and optical signals, which imposes a trade-off between bandwidth and modulation efficiency......

Figure 3-57: S11 Electrical response of the $1 \mathrm{~mm}$ and $0.5 \mathrm{~mm}$ RF transmission line

Figure 3-58: (a) Electrical response of the coplanar waveguide (CPWG) electrodes (S21) with varying length. (b) Radio-frequency propagation losses versus frequency calculated with the cut back method from (a) 147

Figure 3-59: Normalized S21 plotted versus the square root of the frequency. The three electrical transmission curves can be approximated by linear fits, whose equations are given on the right hand side.

Figure 3-60: Microwave propagation losses versus frequency calculated via cutback and through the equation modeling the loss variations caused by the skin effect.

Figure 3-61: Required microwave attenuation coefficient versus frequency for different CPWG length to maintain a maximum of $3 \mathrm{~dB}$ attenuation of the transmission line. The orange dashed line stands for the experimental attenuation coefficient calculated previously.

Figure 3-62: Optical microscope image of the slow wave modulator under test.

The slow wave phase shifters are $500 \mu \mathrm{m}$ long.

Figure 3-63: (a)-(f), Eye diagrams at (a) resp. (d) $10 \mathrm{~Gb} / \mathrm{s}$, (b) resp. (e) $20 \mathrm{~Gb} / \mathrm{s}$ and (d) resp. (e) $30 \mathrm{~Gb} / \mathrm{s}$ resp. (f) and (g) $40 \mathrm{~Gb} / \mathrm{s}$ of the $1 \mathrm{~mm}$ resp. $0.5 \mathrm{~mm}$ long slow wave modulators.

Figure 3-64: Bit-error rate (BER) curves at 10Gb/s and respectively $10 \mathrm{~Gb} / \mathrm{s}$ and $20 \mathrm{~Gb} / \mathrm{s}$ for the $1 \mathrm{~mm}$ and $0.5 \mathrm{~mm}$ long slow wave modulators. 153

Figure 4-1: Schematic of the proposed engineered laterally corrugated waveguide (ELCWG) 157 
Figure 4-2: Band "flattening" process: Blue solid lines and red dashed lines stands for the first three bands before and after hole patterning, respectively. a) and b) describe the unperturbed mode profiles at the band edge while c) and d) depict the altered mode profile after hole patterning.

Figure 4-3: a) Band flattening dependence against hole diameter. b) Group index versus wavelength for varying hole diameter.

Figure 4-4: Average group index (blue solid line) and bandwidth (red dashed line) against hole diameter.

Figure 4-5: real refractive index change $\Delta n$ under different applied drive voltages (a), (a') $\Delta V=-2 \mathrm{~V}$; (b), (b') $\Delta V=-4 \mathrm{~V} ;$ (c), (c') $\Delta V=-6 \mathrm{~V} ;$ (d), (d') $\Delta V=-8 \mathrm{~V}$; for the thin and wide sections of the 1D ELCWG, respectively. The junction is in steady state. Waveguide cross section boundaries are represented by the white plain lines

Figure 4-6: (a) Enhancement factor compared to a $400 \mathrm{~nm}$ rib waveguide and (b) relative modulation efficiency of the ELCWG compared to the LCWG.

Figure 4-7: (a) Schematic of the symmetric MZM based on ELCWG (b) Transmission spectrum for varying applied reverse bias (from $V=0 \mathrm{~V}$ (unbiased) to $V=-10 \mathrm{~V}$ ) of a MZM with a $1430 \mu \mathrm{m}$-long engineered SWPS inserted into both active arms (c) Group index dependence upon wavelength for varying applied reverse bias (from $V=0 \mathrm{~V}$ (unbiased) to $V=-10 \mathrm{~V}$ )

Figure 4-8: (a) Concept schematic of a 1x4 ring resonator-based WDM system with aggregate data rate capabilities of $100 \mathrm{Gbit} / \mathrm{s}$ (4x25 Gbits/s). 4 different wavelengths with $200 \mathrm{GHz}(1.6 \mathrm{~nm})$ channel spacing are injected into each of the 4 ELCWG-based MZI amplitude modulators and multiplexed in a rib waveguide via 4 cascaded ring resonators with slightly different radii to provide resonances centered respectively at the 4 input wavelengths. The 4 modulated signals are then photodetected at the chip output. (b) The blue plain and dashed lines illustrate respectively the DC "on" ('1) "off" ('0') symmetric slow wave MZI's spectra between $0 \mathrm{~V}$ and $6 \mathrm{~V}$, together with the chip output spectra consisting of the 4 modulated channels.

Figure 4-9: SEM images of the fabricated structures (a) ELCWG (b) MZI Yjunction splitter.

Figure 4-10: (a) Normalized transmission spectrum of a $100 \mu \mathrm{m}$ long ELCWG. (b) Normalized transmission spectrum of a $500 \mu \mathrm{m}$ long MZI loaded with a $50 \mu \mathrm{m}$ 
long ELCWG. (c) Experimental group index dependence against wavelength.

Insets show schematics of the integrated structures together with their corresponding transmission spectra.

Figure 4-11: 3D FDTD simulation results of 4 ELCWGs with 4 different waveguides of lengths 5, 10, 20 and $30 \mu \mathrm{m}$. Spectra are normalized and shifted by $10 \mathrm{~dB}$ for clarity. 169

Figure 4-12: (a) 3D FDTD simulation results of tapered and non-tapered LCWGs

(b) Taper dimensions. 170 


\section{List of Tables}

Table 2-1: Summary of the projected Energy per bit consumption of a $50 \Omega$ impedance matched TW MZI modulator meeting CMOS drive voltage requirements $(<1 \mathrm{~V})$.

Table 2-2: State-of-the-art of silicon-based modulators .................................... 50

Table 2-3: Acronym definitions used in Table 2-2 ...................................... 50

Table 3-1: target applications of slow light-based devices and corresponding

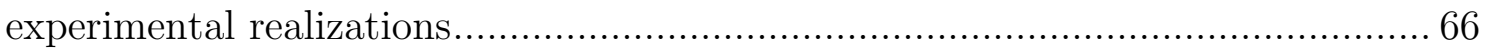

Table 3-2: Summary of the waveguide dimensioning constraints ....................... 80

Table 3-3: Summary of the main FVMS simulation parameters ....................... 82

Table 3-4: Summary of the shallow-etched LCWGs parameters $(\mathrm{h}=50 \mathrm{~nm}) \ldots \ldots \ldots 84$

Table 3-5: Summary of the shallow-etched LCWGs parameters $(\mathrm{h}=100 \mathrm{~nm}) \ldots . . .85$

Table 3-6: Absorption coefficient due to charge carriers as calculated from BPM simulations in a rib waveguide of dimensions $W=300 \mathrm{~nm} \times H=220$ and slab height $=50 \mathrm{~nm}$

Table 3-7: Tolerable temperature span and associated wavelength detuning for varying group index

Table 3-8: Epixfab passive technology standards

Table 3-9: Microwave attenuation at different frequencies for varying CPWG length. 


\section{Acronyms}

LED: Light emitting diode

TIR: Total internal reflection

CMOS: Complementary-metal-oxide semiconductor

VLSI: Very large scale integration

SOI: Silicon-on-insulator

TE: Transverse electric

TM: Transverse magnetic

EO: Electro-optic

EOM: Electro-optic modulator

MBE: Molecular beam epitaxy

VCSEL: Vertical cavity surface emitting laser

FP: Fabry-Pérot

DFP: Distributed feedback laser

MZM: Mach-Zehnder interferometer

MMI: Multi-mode-interferences

RF: Radio-frequency

ISI: Inter-symbol interference

NRZ: Non-return-to-zero

BER: Bit error rate

FEC: Forward error correction

PRBS: Pseudo random bit sequence

SNR: Signal to noise ratio

DC: Direct current

TW: Travelling wave

QCSE: Quantum confined stark effect 
MOS: Metal oxide semiconductor

ER: Extinction ratio

BER: Bit error rate

LCWG: Laterally corrugated waveguide

SOH: Silicon-organic hybrid

PhC: Photonic crystal

FDTD: Finite difference time domain

BPM: Beam propagation method

AC: Alternating current

PWE: Plane wave expansion

SMOF: Standard single mode fiber

MFD: Mode field diameter

DUT: Device under test

ECL: External cavity laser

PC: Polarization controller

VNA: Vectorial network Analyzer

GSG: Ground signal ground

EDFA: Erbium doped fiber amplifier

BPG: Bit pattern generator

DCA: Digital communication analyzer

EA: Error analyzer

OF: Optical filter

CD: Critical dimensions

BOX: Buried oxide

FVMS: Full vectorial mode solver

SWPS: Slow wave phase shifter

FCAL: Free carrier insertion losses

SRSL: Sidewall roughness scattering losses

SW EOM: Slow wave electro-optic modulator

SEM: Scanning electron microscope

FSR: Free spectral range

PBG: Photonic band gap

CPWG: Coplanar waveguide

ELCWG: Engineered laterally corrugated waveguide

QAM: Quadrature amplitude modulation

WDM: Wavelength division-multiplexing 
PON: Passive optical network

AWG: Arrayed waveguide gratings

CW: Continuous wave

ITU: International telecommunication union 


\section{Bibliography}

1. J. D. Meindl, "Interconnect opportunities for gigascale integration," IEEE Micro, 29-35 (May-June 2003).

2. D. Miller, "Device Requirements for Optical Interconnects to Silicon Chips," Proceedings of the IEEE 97, 1166-1185 (2009).

3. G. H. Loh, Y. Xie, and B. Black, "Processor design in 3D stacking technology," IEEE Micro, 31-48 (2007).

4. H. Wang, D. Nezich, J. Kong, and T. Palacios, "Graphene Frequency Multipliers," Electron Device Letters (2008).

5. Y. M. Lin, K. A. Jenkins, A. V. Garcia, J. P. Small, D. B. Farmer, and P. Avouris, "Operation of Graphene Transistors at Gigahertz Frequencies," Nano Letters 9 422-426 (2009).

6. P. Dumon, W. Bogaerts, V. Wiaux, J. Wouters, S. Beckx, J. Van Campenhout, D. Taillaert, B. Luyssaert, P. Bienstman, D. Van Thourhout, and R. Baets, "Low-loss SOI photonic wires and ring resonators fabricated with deep UV lithography," IEEE Photon. Technol. Lett. 16, 1328-1330 (2004).

7. W. Bogaerts, P. Dumon, D. Taillaert, V. Wiaux, S. Beckx, B. Luyssaert, J. Van Campenhout, D. Van Thourhout, and R. Baets, "SOI nanophotonic waveguide structures fabricated with deep UV lithography," Photonics and Nanostructures - Fundamentals and Applications 2, 81-86 (2004).

8. $\quad$ S. M. Sze, "Physics of Semiconductor Devices," John Wiley \& Sons (1981).

9. G. Lifante, "Integrated photonics: fundamentals," Wiley (2003). 
10. J. R. Chelikowsky and M. L. Cohen, "Nonlocal pseudopotential calculations for the electronic structure of eleven diamond and zinc-blende semiconductors," Physical Review B 14, 556 (1976).

11. W. Shockley, "The theory of p-n Junctions in Semiconductors and p-n Junction Transistors," Bell Syst. Tech. J. 28, 457 (1949).

12. S. M. Sze, Physics of Semiconductor Devices (1981).

13. G. T. Reed and A. P. Knights, Silicon Photonics: An Introduction (John Wiley \& Sons, Inc., 2004).

14. N. Dagli, High-Speed Photonic Devices, Series in Optics and Optoelectronics (New York, London, 2007).

15. J. Herrera, (personal communication, 2011).

16. R. A. Soref, and B.R. Bennett, "Electrooptical Effects in Silicon," IEEE J. Quantum Elect. QE-23, 123-129 (1987).

17. M. Asghari and A. V. Krishnamoorthy, "Silicon photonics: Energy-efficient communication," Nat Photon 5, 268-270 (2011).

18. H. Rong, R. Jones, A. Liu, O. Cohen, D. Hak, A. Fang, and M. Paniccia, "A continuous-wave Raman silicon laser," Nature 433, 725-728 (2005).

19. L. Liao, A. Liu, J. Basak, H. Nguyen, M. Paniccia, D. Rubin, Y. Chetrit, R. Cohen, and N. Izhaky, "40 Gbit/s silicon optical modulator for high speed applications," Electronics letters 43(2007).

20. A. Yariv and E. Pochi, Optical waves in crystals (John Wiley \& Sons, Inc, 1984).

21. R. Dekker, N. Usechak, M. Först, and A. Driessen, "Ultrafast nonlinear alloptical processes in silicon-on-insulator waveguides " Journal of Physics D: Applied Physics, 249-271 (2007).

22. T. Vallaitis, S. Bogatscher, L. Alloatti, P. Dumon, R. Baets, M. L. Scimeca, I. Biaggio, F. Diederich, C. Koos, W. Freude, and J. Leuthold, "Optical properties of highly nonlinear silicon-organic hybrid (SOH) waveguide geometries," Opt. Express 17, 17357-17368 (2009).

23. R. S. Jacobsen, K. N. Andersen, P. I. Borel, J. Fage-Pedersen, L. H. Frandsen, O. Hansen, M. Kristensen, A. V. Lavrinenko, G. Moulin, H. Ou, 
C. Peucheret, B. Zsigri, and A. Bjarklev, "Strained silicon as a new electrooptic material," Nature 441, 199-202 (2006).

24. J. Liu, M. Beals, A. Pomerene, S. Bernardis, R. Sun, J. Cheng, L. C. Kimerling, and J. Michel, "Waveguide-integrated, ultralow-energy GeSi electro-absorption modulators," Nat Photon 2, 433-437 (2008).

25. N.-N. Feng, D. Feng, S. Liao, X. Wang, P. Dong, H. Liang, C.-C. Kung, W. Qian, J. Fong, R. Shafiiha, Y. Luo, J. Cunningham, A. V. Krishnamoorthy, and M. Asghari, "30GHz Ge electro-absorption modulator integrated with 3?m silicon-on-insulator waveguide," Opt. Express 19, 7062-7067 (2011).

26. N. Dagli, High-speed photonic devices (Taylor \& Francis group, LLC, 2007).

27. Y.-H. Kuo, Y. K. Lee, Y. Ge, S. Ren, J. E. Roth, T. I. Kamins, D. A. B. Miller, and J. S. Harris, "Strong quantum-confined Stark effect in germanium quantum-well structures on silicon," Nature 437, 1334-1336 (2005).

28. B. Chmielak, M. Waldow, C. Matheisen, C. Ripperda, J. Bolten, T. Wahlbrink, M. Nagel, F. Merget, and H. Kurz, "Pockels effect based fully integrated, strained silicon electro-optic modulator," Opt. Express 19, 17212-17219 (2011).

29. M. Liu, X. Yin, E. Ulin-Avila, B. Geng, T. Zentgraf, L. Ju, F. Wang, and $\mathrm{X}$. Zhang, "A graphene-based broadband optical modulator," Nature 474, 64-67 (2011).

30. G. T. Reed, G. Mashanovich, F. Y. Gardes, and D. J. Thomson, "Silicon optical modulators," Nat Photon 4, 518-526 (2010).

31. A. Liu, R. Jones, L. Liao, D. Samara-Rubio, D. Rubin, O. Cohen, R. Nicolaescu, and M. Paniccia, "A high-speed silicon optical modulator based on a metal-oxide-semiconductor capacitor," Nature 427, 615-618 (2004).

32. L. Liao, D. Samara-Rubio, M. Morse, A. Liu, D. Hodge, D. Rubin, U. Keil, and T. Franck, "High speed silicon Mach-Zehnder modulator," Opt. Express 13, 3129-3135 (2005).

33. Q. Xu, S. Manipatruni, B. Schmidt, J. Shakya, and M. Lipson, "12.5 Gbit/s carrier-injection-based silicon micro-ring silicon modulators," Opt. Express 15, 430-436 (2007). 
34. W. M. Green, M. J. Rooks, L. Sekaric, a. Yurii, and A. Vlasov, "Ultracompact, low RF power, $10 \mathrm{~Gb} / \mathrm{s}$ silicon Mach-Zehnder modulator," Opt. Express 15, 17106-17113 (2007).

35. F. Y. Gardes, A. Brimont, P. Sanchis, G. Rasigade, D. Marris-Morini, L. O'Faolain, F. Dong, J. M. Fedeli, P. Dumon, L. Vivien, T. F. Krauss, G. T. Reed, and J. Martí, "High-speed modulation of a compact silicon ring resonator based on a reverse-biased pn diode," Opt. Express 17, 2198621991 (2009).

36. P. Dong, S. Liao, H. Liang, W. Qian, X. Wang, R. Shafiiha, D. Feng, G. Li, X. Zheng, A. V. Krishnamoorthy, and M. Asghari, "High-speed and compact silicon modulator based on a racetrack resonator with a $1 \mathrm{~V}$ drive voltage," Opt. Lett. 35, 3246-3248 (2010).

37. X. Zheng, J. Lexau, Y. Luo, H. Thacker, T. Pinguet, A. Mekis, G. Li, J. Shi, P. Amberg, N. Pinckney, K. Raj, R. Ho, J. E. Cunningham, and A. V. Krishnamoorthy, "Ultra-low-energy all-CMOS modulator integrated with driver," Opt. Express 18, 3059-3070 (2010).

38. S. Manipatruni, X. Qianfan, B. Schmidt, J. Shakya, and M. Lipson, "High Speed Carrier Injection $18 \mathrm{~Gb} / \mathrm{s}$ Silicon Micro-ring Electro-optic Modulator," in Lasers and Electro-Optics Society, 200\%. LEOS 200\%. The 20th Annual Meeting of the IEEE, 2007), 537-538.

39. D. J. Thomson, F. Y. Gardes, Y. Hu, G. Mashanovich, M. Fournier, P. Grosse, J. M. Fedeli, and G. T. Reed, "High contrast 40Gbit/s optical modulation in silicon," Opt. Express 19, 11507-11516 (2011).

40. F. Y. Gardes, D. J. Thomson, N. G. Emerson, and G. T. Reed, "40 Gb/s silicon photonics modulator for TE and TM polarisations," Opt. Express 19, 11804-11814 (2011).

41. Y. Jiang, W. Jiang, L. Gu, X. Chen, and R. T. Chen, "80-micron interaction length silicon photonic crystal waveguide modulator," Applied physics letters 87, 221105-221103 (2005).

42. H. C. Nguyen, Y. Sakai, M. Shinkawa, N. Ishikura, and T. Baba, "10 Gb/s operation of photonic crystal silicon optical modulators," Opt. Express 19, 13000-13007 (2011). 
43. L. Gu, W. Jiang, X. chen, L. Wang, and R. T. Chen, "High speed silicon photonic crystal waveguide modulator for low voltage operation," Applied physics letters 90, 071105 071101-071103 (2007).

44. C. K. Tang and G. T. Reed, "Highly efficient optical phase modulator in SOI waveguides," Electronics letters 31, 451-452 (1995).

45. X. Chen, Y.-S. Chen, Y. Zhao, W. Jiang, and R. T. Chen, "Capacitorembedded $0.54 \mathrm{pJ} /$ bit silicon-slot photonic crystal waveguide modulator," Opt. Lett. 34, 602-604 (2009).

46. E. Ip, A. P. T. Lau, D. J. F. Barros, and J. M. Kahn, "Coherent detection in optical fiber systems," Opt. Express 16, 753-791 (2008).

47. S. Akiyama, T. Kurahashi, T. Baba, N. Hatori, T. Usuki, and T. Yamamoto, "1-Vpp 10-Gb/s Operation of Slow-Light Silicon MachZehnder Modulator in Wavelength Range of $1 \mathrm{~nm}, "$ IEEE International Conference on Group IV Photonics, 45-47 (2010).

48. A. Brimont, D. J. Thomson, P. Sanchis, J. Herrera, F. Y. Gardes, J. M. Fedeli, G. T. Reed, and J. Martí, "High speed silicon electro-optical modulators enhanced via slow light propagation," Opt. Express 19, 2087620885 (2011).

49. D. J. Thomson, F. Y. Gardes, G. T. Reed, F. Milesi, and J. M. Fedeli, "High speed silicon optical modulator with self aligned fabrication process," Opt. Express 18, 19064-19069 (2010).

50. H.-W. Chen, J. D. Peters, and J. E. Bowers, "Forty Gb/s hybrid silicon Mach-Zehnder modulator with low chirp," Opt. Express 19, 1455-1460 (2011).

51. L. Alloatti, D. Korn, R. Palmer, D. Hillerkuss, J. Li, A. Barklund, R. Dinu, J. Wieland, M. Fournier, J. Fedeli, H. Yu, W. Bogaerts, P. Dumon, R. Baets, C. Koos, W. Freude, and J. Leuthold, "42.7 Gbit/s electro-optic modulator in silicon technology," Opt. Express 19, 11841-11851 (2011).

52. J. A. Dionne, K. Diest, L. A. Sweatlock, and H. A. Atwater, "PlasMOStor: A Metal Oxide Si Field Effect Plasmonic Modulator," Nano Letters 9, 897902 (2009).

53. F. G. Della Corte, S. Rao, G. Coppola, and C. Summonte, "Electro-optical modulation at $1550 \mathrm{~nm}$ in an as-deposited hydrogenated amorphous silicon p-i-n waveguiding device," Opt. Express 19, 2941-2951 (2011). 
54. D. D'Andrea, "CMOS Photonics Today \& Tomorrow Enabling Technology: $\quad$ www.ofcnfoec.org/osa.ofc/media/Default/PDF/2009/09Dandrea.pdf," (2009).

55. J. Fujikata, J. Ushida, M.-B. Yu, Z. ShiYang, D. Liang, P. L. Guo-Qiang, D. L. Kwong, and T. Nakamura, "25 GHz operation of silicon optical modulator with projection MOS structure," in Optical Fiber Communication (OFC), collocated National Fiber Optic Engineers Conference, 2010 Conference on (OFC/NFOEC), 2010), 1-3.

56. C. Gunn, "CMOS Photonics for High-Speed Interconnects," Micro, IEEE 26, 58-66 (2006).

57. M. R. Watts, D. C. Trotter, R. W. Young, and A. L. Lentine, "Ultralow power silicon microdisk modulators and switches," in Group IV Photonics, 2008 5th IEEE International Conference on, 2008), 4-6.

58. J. W. Park, J.-B. You, I. G. Kim, and G. Kim, "High-modulation efficiency silicon Mach-Zehnder optical modulator based on carrier depletion in a PN Diode," Opt. Express 17, 15520-15524 (2009).

59. D. M. Gill, M. Rasras, T. Kun-Yii, C. Young-Kai, A. E. White, S. S. Patel, D. Carothers, A. Pomerene, R. Kamocsai, C. Hill, and J. Beattie, "Internal Bandwidth Equalization in a CMOS-Compatible Si-Ring Modulator," Photonics Technology Letters, IEEE 21, 200-202 (2009).

60. D. M. Gill, S. S. Patel, M. Rasras, T. Kun-Yii, A. E. White, C. YoungKai, A. Pomerene, D. Carothers, R. L. Kamocsai, C. M. Hill, and J. Beattie, "CMOS-Compatible Si-Ring-Assisted Mach\&\#x2013;Zehnder Interferometer With Internal Bandwidth Equalization," Selected Topics in Quantum Electronics, IEEE Journal of 16, 45-52 (2010).

61. L. Tsung-Yang, A. Kah-Wee, F. Qing, S. Jun-Feng, X. Yong-Zhong, Y. Ming-Bin, L. Guo-Qiang, and K. Dim-Lee, "Silicon Modulators and Germanium Photodetectors on SOI: Monolithic Integration, Compatibility, and Performance Optimization," Selected Topics in Quantum Electronics, IEEE Journal of 16, 307-315 (2010).

62. N.-N. Feng, S. Liao, D. Feng, P. Dong, D. Zheng, H. Liang, R. Shafiiha, G. Li, J. E. Cunningham, A. V. Krishnamoorthy, and M. Asghari, "High speed carrier-depletion modulators with $1.4 \mathrm{~V}-\mathrm{cm} \mathrm{V} \pi \mathrm{L} \pi$ integrated on $0.25 \mu \mathrm{m}$ silicon-on-insulator waveguides," Opt. Express 18, 7994-7999 (2010). 
63. Q. Xu, B. Schmidt, S. Pradhan, and M. Lipson, "Micrometre-scale silicon electro-optic modulator," Nature 435, 325-327 (2005).

64. D. Marris-Morini, L. Vivien, J. M. Fédéli, E. Cassan, P. Lyan, and S. Laval, "Low loss and high speed silicon optical modulator based on a lateral carrier depletion structure," Opt. Express 16, 334-339 (2008).

65. G. Rasigade, M. Ziebell, D. Marris-Morini, J.-M. Fédéli, F. Milesi, P. Grosse, D. Bouville, E. Cassan, and L. Vivien, "High extinction ratio 10 Gbit/s silicon optical modulator," Opt. Express 19, 5827-5832 (2011).

66. J. H. Wülbern, S. Prorok, J. Hampe, A. Petrov, M. Eich, J. Luo, A. K. Y. Jen, M. Jenett, and A. Jacob, "40 GHz electro-optic modulation in hybrid silicon?organic slotted photonic crystal waveguides," Opt. Lett. 35, 27532755 (2010).

67. C.-Y. Lin, X. Wang, S. Chakravarty, B. S. Lee, W. Lai, J. Luo, A. K. Y. Jen, and R. T. Chen, "Electro-optic polymer infiltrated silicon photonic crystal slot waveguide modulator with 23 dB slow light enhancement," Applied physics letters 97, 093304-093303 (2010).

68. M. Gould, T. Baehr-Jones, R. Ding, S. Huang, J. Luo, A. K. Y. Jen, J.-M. Fedeli, M. Fournier, and M. Hochberg, "Silicon-polymer hybrid slot waveguide ring-resonator modulator," Opt. Express 19, 3952-3961 (2011).

69. Y. Tang, H.-W. Chen, S. Jain, J. D. Peters, U. Westergren, and J. E. Bowers, "50 Gb/s hybrid silicon traveling-wave electroabsorption modulator," Opt. Express 19, 5811-5816 (2011).

70. Y. A. Vlasov, M. O'Boyle, H. F. Hammann, and S. J. McNab, "Active control of slow light on a chip with photonic crystal waveguides," Nature 483, 65-69 (2005).

71. L. O'Faolain, D. M. Beggs, T. P. White, T. Kampfrath, K. Kuipers, and T. F. Krauss, "Compact Optical Switches and Modulators Based on Dispersion Engineered Photonic Crystals," Photonics Journal, IEEE 2, 404-414 (2010).

72. S. Akiyama, T. Kurahashi, T. Baba, N. Hatori, T. Usuki, and T. Yamamoto, "A 1 V Peak-to-Peak Driven 10-Gbps Slow-Light Silicon Mach-Zehnder Modulator Using Cascaded Ring Resonators," Applied Physics Express 3, 072202 (2010). 
73. K. S. Kunz and R. J. Luebbers, "The Finite Difference Time Domain Method For Electromagnetics," CRC Press, London, UK (1993).

74. "Rsoft Design Group, Inc., 200 Executive Group Blvd. Ossining, NY 10562, www.rsoftdesign.com ".

75. B. Ben Bakir, A. V. de Gyves, R. Orobtchouk, P. Lyan, C. Porzier, A. Roman, and J. M. Fedeli, "Low-Loss (<formula formulatype="inline" $>$ $<$ img src="/images/tex/17012.gif" alt=" $<"></$ formula $>1 \mathrm{~dB}$ ) and Polarization-Insensitive Edge Fiber Couplers Fabricated on 200-mm Silicon-on-Insulator Wafers," Photonics Technology Letters, IEEE 22, 739741 (2010).

76. M. Pu, L. Liu, H. Ou, K. Yvind, and J. M. Hvam, "Ultra-low-loss inverted taper coupler for silicon-on-insulator ridge waveguide," Optics Communications 283, 3678-3682 (2010).

77. D. Vermeulen, S. Selvaraja, P. Verheyen, G. Lepage, W. Bogaerts, P. Absil, D. Van Thourhout, and G. Roelkens, "High-efficiency fiber-to-chip grating couplers realized using an advanced CMOS-compatible Silicon-OnInsulator platform," Opt. Express 18, 18278-18283 (2010).

78. F. Van Laere, G. Roelkens, M. Ayre, J. Schrauwen, D. Taillaert, D. Van Thourhout, T. F. Krauss, and R. Baets, "Compact and Highly Efficient Grating Couplers Between Optical Fiber and Nanophotonic Waveguides," J. Lightwave Technol. 25, 151-156 (2007).

79. A. Liu, L. Liao, D. Rubin, H. Nguyen, B. Ciftcioglu, Y. Chetrit, N. Izhaky, and M. Paniccia, "High-speed optical modulation based on carrier depletion in a silicon waveguide," Optics Express 15, 660-668 (2007).

80. M. Soljacic, S. G. Johnson, F. Shanhui, M. Ibanescu, E. Ippen, and J. D. Joannopoulos, "Photonic-crystal slow-light enhancement of nonlinear phase sensitivity," Journal of the Optical Society of America B (Optical Physics) 19, 2052-2059 (2002).

81. F. Xia, L. Sekaric, and Y. Vlasov, "Ultracompact optical buffers on a silicon chip," Nature Photonics 1, 65-70 (2007).

82. C. Monat, B. Corcoran, D. Pudo, M. Ebnali-Heidari, C. Grillet, M. D. Pelusi, D. J. Moss, B. J. Eggleton, T. P. White, L. O'Faolain, and T. F. Krauss, "Slow Light Enhanced Nonlinear Optics in Silicon Photonic 
Crystal Waveguides," Selected Topics in Quantum Electronics, IEEE Journal of 16, 344-356.

83. J. Li, T. P. White, L. O'Faolain, A. Gomez-Iglesias, and T. F. Krauss, "Systematic design of flat band slow light in photonic crystal waveguides," Opt. Express 16, 6227-6232 (2008).

84. N. Skivesen, A. Têtu, M. Kristensen, J. Kjems, L. H. Frandsen, and P. I. Borel, "Photonic-crystal waveguide biosensor," Opt. Express 15, 3169-3176 (2007).

85. J. G. Castelló, V. Toccafondo, P. Pérez-Millán, N. S. Losilla, J. L. Cruz, M. V. Andrés, and J. García-Rupérez, "Real-time and low-cost sensing technique based on photonic bandgap structures," Opt. Lett. 36, 2707-2709 (2011).

86. J. García-Rupérez, V. Toccafondo, M. J. Bañuls, J. G. Castelló, A. Griol, S. Peransi-Llopis, and Á. Maquieira, "Label-free antibody detection using band edge fringes in SOI planar photonic crystal waveguides in the slowlight regime," Opt. Express 18, 24276-24286 (2010).

87. L. V. Hau, S. E. Harris, Z. Dutton, and C. H. Behroozi, "Light speed reduction to 17 metres per second in an ultracold atomic gas," Nature 397, 594-598 (1999).

88. P.-C. Ku, F. Sedgwick, C. J. Chang-Hasnain, P. Palinginis, T. Li, H. Wang, S.-W. Chang, and S.-L. Chuang, "Slow light in semiconductor quantum wells," Opt. Lett. 29, 2291-2293 (2004).

89. M. González Herráez, K. Y. Song, and L. Thévenaz, "Arbitrary-bandwidth Brillouin slow light in optical fibers," Opt. Express 14, 1395-1400 (2006).

90. M. Notomi, K. Yamada, A. Shinya, J. Takahashi, C. Takahashi, and I. Yokohama, "Extremely large group velocity dispersion of line-defect waveguides in photonic crystal slabs," Phys. Rev. Lett. 87, 253902 (2001).

91. H. Gersen, T. J. Karle, R. J. P. Engelsen, W. Boa, J. P. Korterik, N. F. v. Hulst, T. F. Krauss, and L. Kuipers, "Real-Space Observation of Ultraslow Light in Photonic Crystal Waveguides," Physical review letters 94, 073903 073901-073904 (2005).

92. G. von Freymann, S. John, S. Wong, V. Kitaev, and G. A. Ozin, "Measurement of group velocity dispersion for finite size three-dimensional 
photonic crystals in the near-infrared spectral region," Applied physics letters 86, 053108-053103 (2005).

93. A. Y. Petrov and M. Eich, "Zero dispersion at small group velocities in photonic crystal waveguides," Applied physics letters 85, 4866-4868 (2004).

94. L. H. Frandsen, A. V. Lavrinenko, J. Fage-Pedersen, and P. I. Borel, "Photonic crystal waveguides with semi-slow light and tailored dispersion properties," Opt. Express 14, 9444-9450 (2006).

95. S. Kubo, D. Mori, and T. Baba, "Low-group-velocity and low-dispersion slow light in photonic crystal waveguides," Opt. Lett 32, 2981-2983 (2007).

96. M. D. Settle, R. J. P. Engelen, M. Salib, A. Michaeli, L. Kuipers, and T. F. Krauss, "Flatband slow light in photonic crystals featuring spatial pulse compression and terahertz bandwidth," Optics Express 15, 219-226 (2007).

97. J. D. Joannopoulos, R. D. Meade, and J. N. Winn, Photonic Crystals: Molding the Flow of Light (1995).

98. A. Melloni, F. Morichetti, and M. Martinelli, "Linear and nonlinear pulse propagation in coupled resonator slow-wave optical structures," Optical and Quantum Electronics 35, 365-379 (2003).

99. F. Xia, L. Sekaric, M. O'Boyle, and Y. Vlasov, "Coupled resonator optical waveguides based on silicon-on-insulator photonic wires," Applied physics letters 89, 041122 041121-041123 (2006).

100. A. Brimont, P. Sanchis, J. V. Galan, J. M. Fedeli, A. M. Gutierrez, and J. Marti, "Experimental demonstration of moderately low group velocity in silicon rib photonic Wire Bragg Gratings," Proceedings 23rd Annual Meeting of the IEEE Photonics Society, 713-714 (2009).

101. "ePIXfab: http://www.epixfab.eu/."

102. "Commissariat à l'énergie atomique - Laboratoire des technologies de L'information http://www.minatec.com/."

103. "http://www2.imec.be/."

104. "Valencia Nanophotonics Techology Center, Universitat Politécnica de Valencia: www.ntc.upv.es."

105. S. Hughes, L. Ramunno, J. F. Young, and J. E. Sipe, "Extrinsic Optical Scattering Loss in Photonic Crystal Waveguides: Role of Fabrication 
Disorder and Photon Group Velocity," Physical review letters 94, 033903 (2005).

106. W. Bogaerts, P. Bienstman, D.Taillaert, R. Baets, and D. D. Zutter, "Outof-plane scattering in 1-D photonic crystal slabs," Optical and Quantum Electronics 34, 195-203 (2002).

107. E. Kuramochi, M. Notomi, S. Hughes, A. Shinya, T. Watanabe, and L. Ramunno, "Disorder-induced scattering loss of line-defect waveguides in photonic crystal slabs," Physical Review B 72, 161318 (2005).

108. L. O'Faolain, T. P. White, D. O'Brien, X. Yuan, M. D. Settle, and T. F. Krauss, "Dependence of extrinsic loss on group velocity in photonic crystal waveguides," Opt. Express 15, 13129-13138 (2007).

109. G. Jaime, M. Alejandro, and M. Javier, "Influence of Group Velocity on Roughness Losses for 1D Periodic Structures," in Slow and Fast Light, OSA Technical Digest (CD) (Optical Society of America, 2007), JTuA4.

110. M. L. Povinelli, S. G. Johnson, and J. D. Joannopoulos, "Slow-light, bandedge waveguides for tunable time delays," Optics Express 13, 7045-7059 (2005).

111. L. Ofaolain, X. Yuan, D. McIntyre, S. Thoms, H. Chong, R. M. De la Rue, and T. F. Krauss, "Low-loss propagation in photonic crystal waveguides," Electronics letters 42, 1454-1455 (2006).

112. M. Gnan, S. Thoms, D. S. Macintyre, R. M. De La Rue, and M. Sorel, "Fabrication of low-loss photonic wires in silicon-on-insulator using hydrogen silsesquioxane electron-beam resist," Electron. Lett 44, 115-116 (2008).

113. Y. A. Vlasov and S. J. McNab, "Coupling into the slow light mode in slabtype photonic crystal waveguides," Opt. Lett. 31, 50-52 (2006).

114. A. Brimont, F. Y. Gardes, P. Sanchis, D. Marris-Morini, P. Dumon, J. M. Fedeli, L. O'Faolain, W. Bogaert, L. Vivien, J. Martí, G. T. Reed, and T. F. Krauss, "Design of a micro-ring resonator electro-optical modulator embedded in a reverse biased PN junction," Proceedings 14th European Conference on Integrated Optics 321-324 (2008).

115. "SILVACO International, 4701 Patrick Henry Drive, Bldg 1, Sant Clara, California 94054, USA. http://www.silvaco.com." 
116. A. Brimont, J. Vicente Galán, J. Maria Escalante, J. Martí, and P. Sanchis, "Group-index engineering in silicon corrugated waveguides," Opt. Lett. 35, 2708-2710.

117. Intel®, "Intel® Core ${ }^{\mathrm{TM}} 2$ Extreme Quad- Core Processor and Intel® Core ${ }^{\mathrm{TM}} 2$ Quad Processor Thermal and Mechanical Design Guidelines: ftp://download.intel.com/design/processor/designex/315594.pdf," (2009).

118. X. Zheng, J. Lexau, Y. Luo, H. Thacker, T. Pinguet, A. Mekis, G. Li, J. Shi, P. Amberg, N. Pinckney, K. Raj, R. Ho, J. E. Cunningham, and A. V. Krishnamoorthy, "Ultra-low-energy all-CMOS modulator integrated with driver," Opt. Express 18, 3059-3070.

119. B. Guha, B. B. C. Kyotoku, and M. Lipson, "CMOS-compatible athermal silicon microring resonators," Opt. Express 18, 3487-3493 (2010).

120. J. Teng, P. Dumon, W. Bogaerts, H. Zhang, X. Jian, X. Han, M. Zhao, G. Morthier, and R. Baets, "Athermal Silicon-on-insulator ring resonators byoverlaying a polymer cladding on narrowedwaveguides," Opt. Express 17, 14627-14633 (2009).

121. J. M. Brosi, C. Koos, L. C. Andreani, M. Waldow, J. Leuthold, and W. Freude, "High Speed low-voltage electro-optic modulator with a polymer infiltrated silicon photonic crystal waveguide," Opt. Express 16, 4177-4191 (2008).

122. A. Brimont, P. Sanchis, and J. Martí, "Strong electro-optical modulation enhancement in a slow wave corrugated waveguide," Optics Express 17, 9204-9211 (2009).

123. "HELIOS, Photonics Electronics functional integration on CMOS", retrieved http://www.helios-project.eu/.

124. "Technology paper standard LETI: www.epixfab.eu/uploads/media/LETI 05 technical standard.pdf."

125. "Technology paper standard IMEC: www.epixfab.eu/uploads/media/IMEC 193 01 technical v2.pdf."

126. P. Dumon, W. Bogaerts, V. Wiaux, J. Wouters, S. Beckx, J. V. Campenhout, D. Taillaert, B. Luyssaert, P. Bienstman, D. V. Thourhout, and R. Baets, "Low-loss SOI photonic wires and ring resonators fabricated with deep UV lithography," Photonics Technology Letters, IEEE 16, 13281330 (2004). 
127. P. Sanchis, A. Brimont, A. Gutierrez, and M. Aamer, "A method for experimentally estimating the insertion losses of slow-light photonic waveguiding structures," submitted (2011).

128. A. C. Reyes, S. M. El-Ghazaly, S. Dorn, M. Dydyk, and D. K. Schroder, "Silicon as a microwave substrate," in Microwave Symposium Digest, 1994., IEEE MTT-S International, 1994), 1759-1762 vol.1753.

129. M. D. Settle, R. J. P. Engelen, M. Salib, A. Michaeli, L. Kuipers, and T. F. Krauss, "Flatband slow light in photonic crystals featuring spatial pulse compression and terahertz bandwidth," Opt. Express 15, 219-226 (2007).

130. A. Brimont, J. Vicente Galán, J. Maria Escalante, J. Martí, and P. Sanchis, "Group-index engineering in silicon corrugated waveguides," Opt. Lett. 35, 2708-2710 (2010).

131. T. Baba, "Slow light in photonic crystals," Nat Photon 2, 465-473 (2008).

132. A. Brimont, J. Marti, and P. Sanchis, "Slow wave and resonator structures to enhance silicon photonic modulators performance," Accepted in Proceedings 2nd Conferencia Española de Nanofotónica (2010).

133. M. Soljacic and J. D. Joannopoulos, "Enhancement of nonlinear effects using photonic crystals," Nature Materials 3, 211-219 (2004).

134. D. M. Beggs, T. P. White, L. O'Faolain, and T. F. Krauss, "Ultracompact and low-power optical switch based on silicon photonic crystals," Opt. Lett 33, 147-149 (2008).

135. L. O'Faolain, S. Schulz, D. M. Beggs, T. P. White, A. D. Falco, A. Samarelli, M. Sorel, R. M. D. L. Rue, F. Morichetti, A. Canciamilla, A. Melloni, and T. F. Krauss, "Low loss dispersion engineered photonic crystal waveguides for optical delay lines," Proceedings 6th International IEEE Conference on Group IV Photonics, 40-42 (2009).

136. O. Khayam and H. Benisty, "General recipe for flatband in photonic crystal waveguides," Opt. Express 17, 14634-14648 (2009).

137. W. Bogaerts, P. Dumon, D. V. Thourhout, D. Taillaert, P. Jaenen, J. Wouters, S. Beckx, V. Wiaux, and R. G. Baets, "Compact WavelengthSelective Functions in Silicon-on-Insulator Photonic Wires," Selected Topics in Quantum Electronics, IEEE Journal of 12, 1394-1401 (2006). 
138. X. Zheng, I. Shubin, G. Li, T. Pinguet, A. Mekis, J. Yao, H. Thacker, Y. Luo, J. Costa, K. Raj, J. E. Cunningham, and A. V. Krishnamoorthy, "A tunable 1x4 silicon CMOS photonic wavelength multiplexer/demultiplexer for dense optical interconnects," Opt. Express 18, 5151-5160 (2010). 